\title{
Statistical Study of Magnetic Field Reversals in Geodynamo Models and Paleomagnetic Data
}

\author{
Dissertation \\ zur Erlangung des mathematisch-naturwissenschaftlichen Doktorgrades \\ "Doctor rerum naturalium" \\ der Georg-August-Universität Göttingen \\ im Promotionsprogramm PROPHYS \\ der Georg-August University School of Science (GAUSS)
}

vorgelegt von

Domenico Giovanni Meduri

aus Reggio di Calabria, Italien

Göttingen, 2014 
Betreuungsausschuss

Prof. Dr. Andreas Tilgner

Institut für Geophysik, Georg-August-Universität Göttingen, Germany

Prof. Dr. Ulrich Christensen

Max-Planck-Institut für Sonnensystemforschung, Göttingen, Germany

Dr. Johannes Wicht

Max-Planck-Institut für Sonnensystemforschung, Göttingen, Germany

Mitglieder der Prüfungskommision

Referent: Prof. Dr. Andreas Tilgner

Institut für Geophysik, Georg-August-Universität Göttingen, Germany

Korreferent: Dr. Johannes Wicht

Max-Planck-Institut für Sonnensystemforschung, Göttingen, Germany

Weitere Mitglieder der Prüfungskommission:

Prof. Dr. Ulrich Christensen

Max-Planck-Institut für Sonnensystemforschung, Göttingen, Germany

Prof. Dr. Dominik Schleicher

Institut für Astrophysik, Georg-August-Universität Göttingen, Germany

Prof. Dr. Ansgar Reiners

Institut für Astrophysik, Georg-August-Universität Göttingen, Germany

Prof. Dr. Wolfram Kollatschny

Institut für Astrophysik, Georg-August-Universität Göttingen, Germany

Tag der mündlichen Prüfung: 29 Oktober 2014 


\section{Bibliografische Information der Deutschen Nationalbibliothek}

Die Deutsche Nationalbibliothek verzeichnet diese Publikation in der Deutschen Nationalbibliografie; detaillierte bibliografische Daten sind im Internet über http: //dnb . d-nb . de abrufbar.

ISBN 978-3-944072-11-1

uni-edition $\mathrm{GmbH} 2015$

http://www.uni-edition.de

(C) Domenico Giovanni Meduri

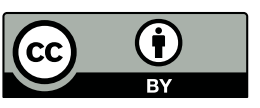

This work is distributed under a

Creative Commons Attribution 3.0 License

Printed in Germany 



\section{Contents}

$\begin{array}{ll}\text { Summary } & 7\end{array}$

1 Introduction 9

1.1 Historical background . . . . . . . . . . . . . . . . . . 9 9

1.2 The Earth's interior and the quest for dynamo action . . . . . . . . 10

1.3 The recent geomagnetic field . . . . . . . . . . . . . . . . 11

1.4 The paleomagnetic field . . . . . . . . . . . . . . . . . . . . . . 14

1.4 .1 Rock magnetism . . . . . . . . . . . . . . . . . . . 15

1.4 .2 Observational evidences for field reversals . . . . . . . . . . . 16

$1.4 .3 \quad$ Geomagnetic polarity transitions . . . . . . . . . . . . . . 17

1.4 .4 The geomagnetic polarity time scale . . . . . . . . . . . . . 22

1.4.5 Statistical analyses of geomagnetic reversal occurrences . . . . 26

2 The geodvnamo: theory and numerical modelling 31

2.1 Hydrodynamics . . . . . . . . . . . . . . . . . . . . . . 31

2.2 Thermodynamic equations ... . . . . . . . . . . . . . 33

2.3 Equations for the convective perturbations . . . . . . . . . . . 36

2.4 Rotating frame of reference . . . . . . . . . . . . . . . . . . . 41

2.5 The magnetic induction equation $\ldots \ldots \ldots \ldots \ldots$. . . . . . . . . . 42

2.6 The non-dimensionalization $\ldots \ldots \ldots \ldots$

2.7 Boundary conditions $\ldots \ldots \ldots \ldots \ldots$

2.8 The numerical method . . . . . . . . . . . . . . . . 51

3 Probability theory and statistical tools 55

3.1 The concept of probability $\ldots \ldots \ldots \ldots \ldots$. . . . . . . . . 55

3.1 .1 Interpretation of probability $\ldots \ldots \ldots \ldots$. . . . . . 57

3.2 Fundamentals of probability theory $\ldots \ldots \ldots \ldots \ldots \ldots$

3.3 Survival and hazard functions . . . . . . . . . . . . . . . . . . . 59

3.4 Statistical models and theoretical distributions . . . . . . . . . . . 61

3.4 .1 Theoretical distributions . . . . . . . . . . . . . . . . 61

3.4 .2 The Poisson process . . . . . . . . . . . . . . . . . . 72

3.5 The method of maximum likelihood . . . . . . . . . . . . . 75

3.5.1 Transformation invariance of the ML estimator . . . . . . . . 76

3.5 .2 Desirable properties of estimators . . . . . . . . . . . 76

3.5 .3 Asymptotic behavior of the ML estimator . . . . . . . . . . . 79

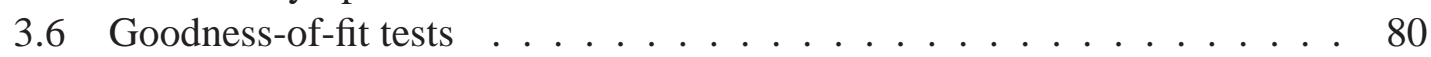


3.6.1 Kolmogorov-Smirnov test . . . . . . . . . . . . . . 80 80

3.6.2 Anderson-Darling test . . . . . . . . . . . . . . 82

3.7 The Bavesian approach . . . . . . . . . . . . . . . . 82

3.7 .1 Parameter estimation . . . . . . . . . . . . . 82

3.7 .2 Choice of priors . . . . . . . . . . . . . . . . . . . . . . . . . . . . . . 84

3.7 .3 Model selection . . . . . . . . . . . . . . . . . . 85

3.7 .4 A built-in Occam's razor . . . . . . . . . . . . . . . . 87

$4 \quad$ Results from geomagnetic polarity time scales $\quad 89$

4.1 Selection of geomagnetic polarity time scales . . . . . . . . . . . 89

4.2 Distribution of paleomagnetic polarity intervals . . . . . . . . . . . . . . 94

5 Results from geodvnamo simulations $\quad 103$

5.1 Models description and comparison with the Earth . . . . . . . . . . 103

5.2 Field evolution during polarity transitions . . . . . . . . . . . . . . . 113

5.3 Definition of events and filtering algorithm . . . . . . . . . . . . . 116 5.3.1 Selection of grand excursions . . . . . . . . . . . . . 120

5.4 Distribution of the durations of stable polarity intervals, reversals and excursions 122

5.4.1 Stable polarity intervals and occurrence of reversals . . . . . . . 123

5.4 .2 Durations of reversals . . . . . . . . . . . . . . . . 134

5.4 .3 Durations of grand excursions . . . . . . . . . . . . . . . 142

5.4.4 Equivalence of reversals and grand excursions . . . . . . . . . . . 145

5.5 Stable polarity intervals from a paleomagnetic point of view . . . . . . . 148

6 Conclusions and outlook 155

\begin{tabular}{ll}
\hline Bibliography & 161
\end{tabular}

\begin{tabular}{lr}
\hline Acknowledgements & 169
\end{tabular}

$\begin{array}{ll}\text { Scientific contributions } & 171\end{array}$

\begin{tabular}{ll}
\hline Curriculum Vitae & 173
\end{tabular} 


\section{Summary}

The main magnetic field of the Earth is generated by convective motions in the liquid iron core. The field shows complex variations on a broad range of time scales. Shorter scales in the range of years are likely related to wave phenomena (Gillet et al. 2010) while decadal to centennial variations reflect the convective flow dynamics (Christensen et al. 2012). Much longer time scales are mostly associated to global polarity reversals of the dipole field component which typically last $4-5$ thousand years (Merrill and McFadden 1999). Paleomagnetic measurements of sea-floor magnetic anomalies document several hundred polarity switches in the last 180 million years of geomagnetic history with an average rate of $2-3$ per million years (Jacobs 1994). Reversals can therefore be regarded as rare and almost instantaneous events on geologic time scales.

Stable polarity epochs are also punctuated by another type of events, known as excursions. During such events, the magnetic pole may venture into the opposite hemisphere for periods comparable to the duration of reversals but the opposite polarity is never firmly established. Several excursions are documented for the last few million years of geomagnetic history but only some of them have been correlated globally (Gubbins 1999, Laj and Channell 2007).

Paleomagnetic studies indicate that the average reversal frequency has changed over time scales of the order of tens to hundred million years (Biggin et al. 2012). The Cretaceous normal superchron (CNS) is a particularly long stable polarity epoch of about 35 million years which roughly started 118 million years before present. An increasing trend in the geomagnetic reversal frequency from the end of the CNS to the present has been reported, while particularly high reversal rates before this event have also been found. The question whether such variations are of external origin, most often identified with the mantle influence, or reflect the dynamics of the strongly non-linear internal dynamo processes is still a matter of debate.

Self-consistent magneto-hydrodynamic (MHD) simulations successfully reproduce many features of the Earth's magnetic field. Several of these numerical dynamo models also experience dipole field reversals with characteristics similar to paleomagnetic observations (Amit et al. 2010). Due to the inherent complexity of the non-linear processes involved, detailed studies of simulated polarity transitions only partially helped in determining the fluid dynamic mechanisms responsible for reversals and excursions (see, e.g., Wicht and Olson 2004, Aubert et al. 2008). The statistical characterization of these events aims to provide robust constraints on our knowledge of the physical processes underlying their occurrence.

This work presents a systematic statistical study of the reversals and excursions in two dynamo models with different Ekman numbers and Rayleigh numbers. Both models have been run for several thousand magnetic diffusion times and undergo several hundred 
reversals, thus representing by far the longest numerical dynamo records to date. Different distribution functions, used in previous related studies and describing diverse random processes, have been tested for modeling the statistics of stable polarity interval durations and the durations of reversals and excursions. We quantified the relative quality of the individual models in describing the data using a Bayesian approach.

The occurrence of reversals is best described by a (stationary) Poisson process in the dynamo models analyzed here. The number of reversals and excursions is very similar in the numerical dynamo with the lower Ekman number and the duration of both types of events follows a gamma distribution. We therefore conclude that reversals and excursions are expressions of the same underlying process. This process consists of three stages: first the dipole moment decreases significantly in order to allow for large angular deviations of the magnetic pole. The dynamo tends then to linger for a relatively brief period (compared to the free dipole decay time) at low dipole intensities characterized by a more complex multipolar field. Finally the dipole moment recovers and it seems a matter of chance whether the normal or reverse polarity is amplified, thus leading to an excursion or a reversal respectively.

We also addressed the question whether the statistic of the simulated reversals reproduces the Earth's behavior. To this end, we analyzed the most recent paleomagnetic reversal chronologies. Log-normal and log-logistic distributions, both characterized by heavy tails, best describe the chron durations with similar posterior model probabilities. When simulating the limited time resolution of the paleomagnetic record (missing shorter events, non-resolved reversals) the polarity epochs in the numerical simulation show similar statistics. Our analysis thus suggests that the deviation from poissonianity observed in the paleomagnetic record could be the consequence of the limited data quality rather than the signature of a different statistical process. 


\section{Introduction}

This chapter summarizes important observational aspects of the present and past geomagnetic field. After a brief historical introduction and a description of the Earth's interior structure (Sections 1.1 and 1.2), the present geomagnetic field morphology as inferred from global model reconstructions is described in Section 1.3. Insights from paleomagnetic studies on dipole field reversals (and excursions) are discussed in Section 1.4 .

\subsection{Historical background}

The first archeomagnetic artifact potentially representing a primitive compass dates back to 1000 BC and belongs to the Olmec civilization in Mexico (Carlson 1975). This shaped bar of lodestone, a naturally magnetized piece of magnetite, was most probably used as a device for divination. By the 11th century, the Chinese used a magnetized needle for navigational orienting and therefore deserve the credit for discovering Earth's magnetism. The scientific study of the Earth's magnetic field began in the 15th century with early investigations of its properties during the period of global exploration. However, the origin of the geomagnetic field remained a mystery for a long time. Gilbert (1600) was the first to suggest that the geomagnetic field is generated within the Earth itself. In his experiments, Gilbert demonstrated that the pattern of field lines on a uniformly magnetized sphere approximates the directions of the compass needle observed at the Earth's surface. He then concluded that the Earth behaves like a giant magnet.

Variations of the geomagnetic field on time scales of about one year or more (secular variation) were first observed in the late 17th century. Collecting several measurements of the magnetic field declination at different locations, Halley (1683) showed that a large part of the secular variation could be explained by a westward drift of the field. To explain the drift, Halley proposed a model of the Earth's interior consisting of concentric shells of magnetic material rotating in the prograde sense, but with the internal regions spinning slightly slower than the exterior ones and thus causing the magnetic field to drift systematically westward as seen from the Earth's surface (Halley 1692). Remarkable similarities with our current understanding of the Earth's interior, i.e. a layered structure and the association of internal motions with magnetic field variations, were therefore born. However, the origin of the magnetic field was still incorrectly regarded in a permanently magnetized solid inner core.

Collecting observations recorded during sea voyages, Halley also published a map showing lines of constant declination (Halley 1701). This represents the first magnetic chart and stimulated later generations of scientists in the study of geomagnetism through the record of global geophysical data. In the 1830s, Carl-Friedrich Gauss and Alexan- 
der von Humboldt founded what can be regarded as the first international geophysical organization: the "Göttinger Magnetischer Verein". The aim of such organization was to coordinate the systematic measurement of the geomagnetic field declination among tens of observatories located worldwide in order to study field variations. More information on the early history of geomagnetism can be found in Merrill (1998, Chapter 1), Stern (2002) and Kono (2007).

\subsection{The Earth's interior and the quest for dynamo action}

Somewhat surprisingly, the belief of Earth as a permanently magnetized body survived until the beginning of the 20th century. Apparently solved the problem of the geomagnetic field origin, the interest of scientists focussed on explaining the magnetic field of the Sun. In this attempt, Larmor (1919) posed the conceptual basis of modern hydromagnetic dynamo theory. Larmor suggested that, in the presence of an initial magnetic field, motions of electrically conducting fluids within a rotating body might generate induced currents able to amplify and sustain the field against ohmic dissipation. Larmor's hypothesis contains the key ingredients for self-sustained dynamo action and is a simplified, but still valid, description of the basic mechanism producing the magnetic field of the Earth and many other cosmic bodies.

By inference from seismological observations, we know that the Earth's interior structure is layered into spherical shells (Figure 1.1) and differs from what Gilbert and Halley imagined more than three centuries ago. The outermost layers are a silicate solid crust

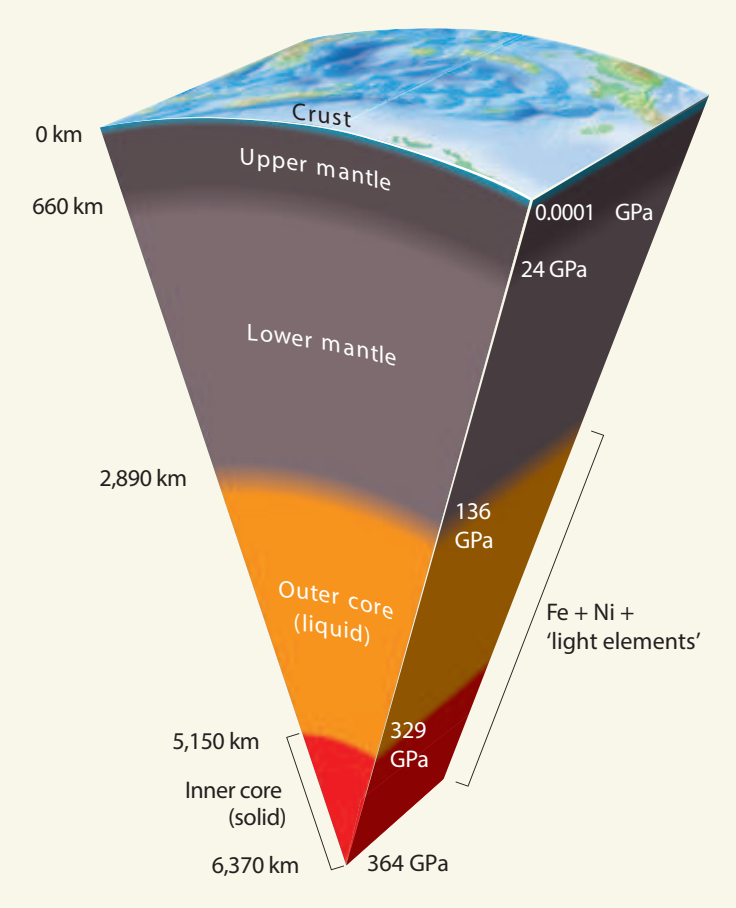

Figure 1.1: Cross-section of the Earth's interior. Depths for the various regions are indicated on the left and corresponding pressures on the right. From Duffy (2011). 
and a highly viscous mantle extending to a depth of about $2890 \mathrm{~km}$. Because of the high pressure and temperature conditions, the silicate rocks composing the mantle undergo viscous deformation and convective material circulation is present on very long time scales. The mantle encloses the core which is composed by a solid part (inner core) and a liquid one (outer core). The inner core radius is $r_{i}=1220 \mathrm{~km}$ and the temperature at the interface with the outer core (inner-core boundary) is $T_{i}=5500 \mathrm{~K}$. The outer core has a thickness $d=2260 \mathrm{~km}$ and the core-mantle boundary (CMB) temperature is $T_{o}=4000 \mathrm{~K}$. The Earth's core is mainly constituted of iron, while the second most abundant element is nickel. Along with $\mathrm{Fe}$ and $\mathrm{Ni}$, light elements (particularly $\mathrm{S}, \mathrm{O}, \mathrm{Si}$ ) are also present and account for about $8 \%$ of the core mass (Duffy 2011). Since the Curie temperature of iron is of about $750 \mathrm{~K}$ and is exceeded a few tens of kilometers below the crust, Gilbert's conception of the Earth as a permanently magnetized body is certainly not correct.

According to the magnetic remanence of certain rocks (see, e.g., Usui et al. 2009), evidences for the existence of a magnetic field go back to at least $3.45 \mathrm{Ga}$ and possibly are as old as the core itself. Assuming that the geomagnetic field has been generated at the time of core formation and no physical processes helped to maintain it, the field would simply decay by ohmic diffusion. The $e$-folding time of a large-scale dipole field diffusing by ohmic processes within the core is $\tau_{d}=r_{o}^{2} / \pi^{2} \eta$, where $r_{o}$ is the outer core radius and $\eta$ the magnetic diffusivity. Using recent estimates of the electrical conductivity at Earth's core conditions (Pozzo et al. 2012), the free dipole decay time is $\tau_{d} \approx 56 \mathrm{kyr}$ which is more than 5 orders of magnitude shorter than the first evidence of the ancient geomagnetic field. This represents a second argument against the hypothesis of a permanent magnetization of the Earth's core and points towards the necessity of a regeneration process able to sustain the magnetic field against ohmic decay. The small enough viscosity of the iron-rich liquid outer core (comparable to that of liquid water) permits turbulent convective flows to develop. These complex fluid motions induce electric currents and therefore magnetic fields exactly as envisioned by Larmor (1919). The variety of dynamical processes occurring in the core which are able to transform the kinetic energy of fluid flows into electromagnetic energy are known as the geodynamo.

The ultimate long-term energy source for the geodynamo comes from the secular cooling of the core. As the core cools down, the base of the liquid core crystallizes at the inner-core boundary (ICB) thus causing the inner core to grow. Two important sources of buoyancy are produced in this process. One source of buoyancy is generated by the light elements which cannot be dissolved efficiently anymore at the ICB and drive the socalled compositional convection. The other source is due to the latent heat release. Since the CMB heat-flow exceeds the amount of heat conducted along the adiabat throughout the core, thermal convection can take place. Heat released by the radioactive decay of unstable elements (such as $\mathrm{K}$, Th and $\mathrm{U}$ ) also contributes to the total energy budget.

\subsection{The recent geomagnetic field}

The term geomagnetic field generally designates the magnetic field produced by all the sources within the solid Earth and its atmosphere, up to the magnetopause. Sources of internal origin include rocks which have been magnetized in the past (permanent magnetization) but also retaining the additional magnetization due to the present ambient field 
(induced magnetization). Magnetized rocks lie in the uppermost (solid) layers of the Earth and produce the crustal field. All other sources are ultimately electric currents and are generated inside the metallic core, in the mantle and crust, in the oceans and finally in the ionosphere and magnetosphere.

The most intense field, with a mean amplitude of about $45 \mu \mathrm{T}$ at the Earth's surface, is known as the main field and is produced within the core through a self-sustaining dynamo process. The crustal field is weaker than the main field and shows strong spatial variations from fractions of a nT to few $\mu \mathrm{T}$. The sum of the main field and the crustal field is often referred to as the internal field. As already mentioned, the Earth's magnetic field has also sources above the neutral atmosphere which constitute the so-called external field. Electric currents produced by the motion of charged particles in the ionosphere and magnetosphere are the main sources of the external field. As measured at the Earth's surface, the external field intensity is of the order of few tens of nT but it can occasionally be up to two orders of magnitude stronger. The typical time scales of the external field variations range from a fraction of a second to several days and are thus significantly faster than the internal field variations.

Since the geomagnetic field is a vector field, it can be described at any given point of the Earth's surface by the three orthogonal components $X$ (pointing in the geographic north direction), $Y$ (pointing eastward) and $Z$ (pointing downward). The horizontal direction $H=\sqrt{X^{2}+Y^{2}}$ is aligned in the direction of the compass needle, while the total field intensity is $F=\sqrt{X^{2}+Y^{2}+Z^{2}}$.

An alternative characterization of the field is generally used in paleomagnetism (see Section 1.4). Instead of its vector components $(X, Y, Z)$, the geomagnetic field is specified at any given point by the field intensity $F$ and two angular measures: the declination $D$ and the inclination I. The declination is defined as the angle between $H$ and the geographic north, thus $D=\arctan Y / X$. The inclination is the angle between the horizontal plane and the field vector $\mathbf{F}$, thus $I=\arctan Z / H$.

Carl-Friedrich Gauss proposed a useful decomposition of the magnetic field $\mathbf{B}$ on a sphere which is still used today to represent the Earth's magnetic field. Since the lower Earth's atmosphere is a very poor electric conductor, no currents can flow in this region and the electric current density $\mathbf{J}$ can be assumed to vanish. By means of Ampère's law, $\mathbf{B}$ is then conservative $(\nabla \times \mathbf{B}=0)$ and can therefore be expressed as the gradient of a scalar potential $V$ :

$$
\mathbf{B}=-\nabla V .
$$

Using the solenoidal property of the magnetic field $(\nabla \cdot \mathbf{B}=0)$, we are lead to the Laplace's equation

$$
\nabla^{2} V=0 .
$$

In a spherical system of coordinates and assuming that the source currents reside in the interior of a spherical surface of radius $r=a$ (internal sources only), the solution of Laplace's equation (1.2) reads (see, e.g., Backus et al. 1996)

$$
V(r, \theta, \phi)=a \sum_{\ell=1}^{\infty}\left(\frac{a}{r}\right)^{\ell+1} \sum_{m=0}^{\ell}\left(g_{\ell}^{m} \cos m \phi+h_{\ell}^{m} \sin m \phi\right) P_{\ell}^{m}(\cos \theta),
$$

where $\theta$ and $\phi$ are colatitude and longitude respectively, $P_{\ell}^{m}$ is the Schmidt quasi-normalized associated Legendre function of degree $\ell$ and order $m$ (both being integers) and $g_{\ell}^{m}$ and $h_{\ell}^{m}$ 

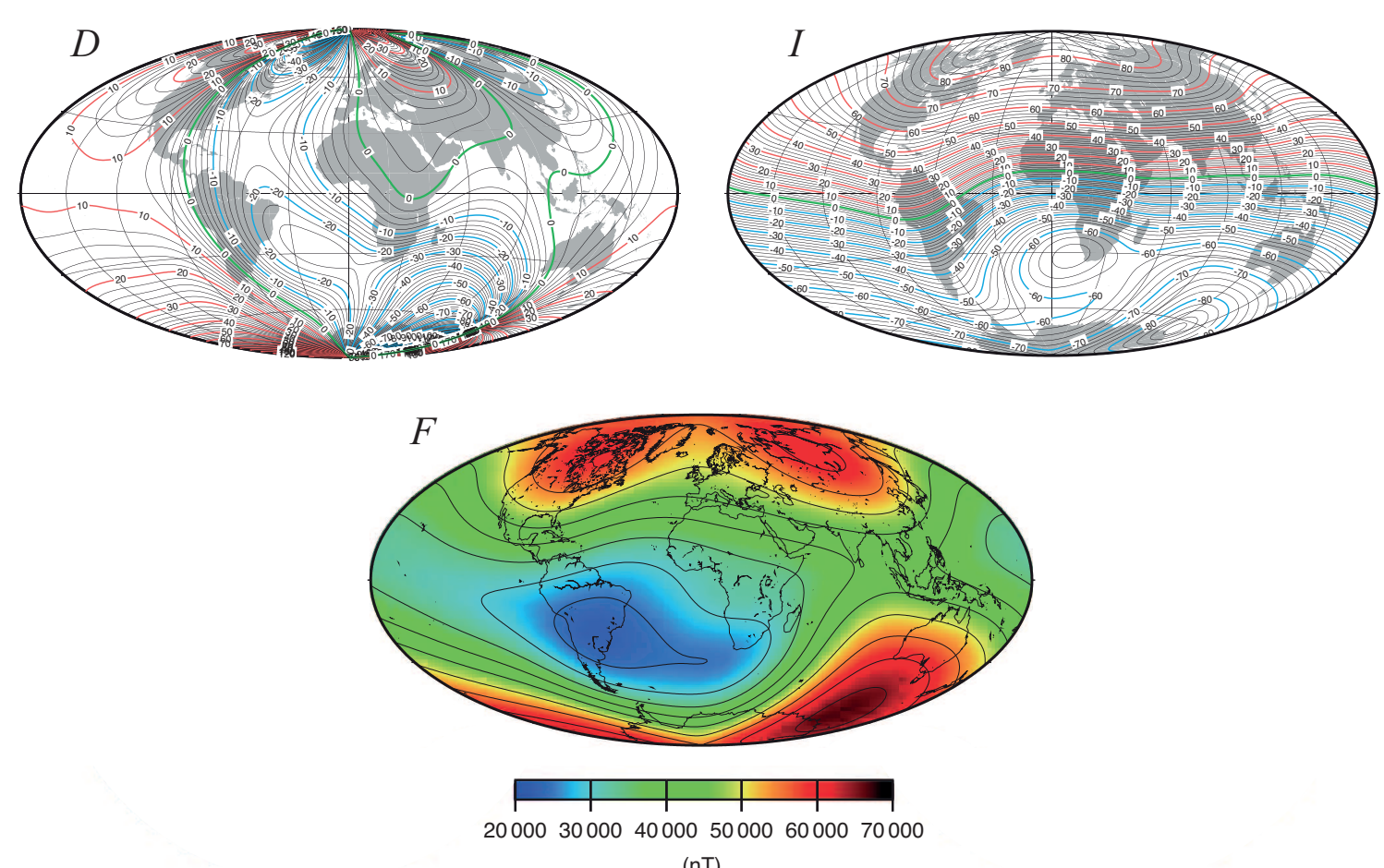

(nT)

Figure 1.2: Maps of the geomagnetic field declination $D$ (top left), inclination $I$ (top right) and intensity $F$ (bottom) at the Earth's surface in year 2005 as reconstructed by the CHAOS model (Olsen et al. 2006). Declination and inclination values are given in degrees. Iso-contour lines of field intensity are in steps of $5000 \mathrm{nT}$. Adapted from Olsen et al. (2007).

are constants known as the Gauss coefficients. If external sources are present, the solution of (1.2) contains also terms proportional to $r^{\ell}$.

At present, the Earth's magnetic field is continuously monitored by about 150 geomagnetic observatories placed worldwide. Since the first space-borne measurements taken by the Sputnik 3 in 1958, satellite magnetometers provided field measurements covering the entire Earth. Both ground-based and satellite observations are used to produce global models of the recent Earth's magnetic field. These models aim at describing the field of internal origin and thus rely on the spherical harmonic representation (1.3). Each of these models is truncated at a certain degree $L$, depending on the number and quality of the data used, and some time dependence in the Gauss coefficients is introduced to account for the fact that the field of internal origin varies with time. Certain corrections are furthermore required to get rid of externally induced fields. We refer to Olsen et al. (2007) for a comprehensive description of the most recent global geomagnetic field models.

Figure 1.2 shows the main magnetic field at the Earth's surface in year 2005 as given by the CHAOS model of Olsen et al. (2006). Maps of the magnetic field declination $D$, inclination $I$ and field intensity $F$ are given in the top left, top right and bottom panels respectively. Only degrees up to $L=13$ have been used to reconstruct the field since higher degrees host crustal contributions. As discussed in Section 1.1, the most simple approximation of the geomagnetic field is that of a geocentric axial dipole and was proposed by Gilbert (1600). In the case of a pure axial dipole field the compass needle points to the 
geographic north all over the globe, meaning that the declination is zero everywhere. Furthermore, the inclination $I$ is $+90^{\circ}$ at the geographic north pole, $-90^{\circ}$ at the geographic south pole and $0^{\circ}$ at the equator and lines of equal inclination are parallel to the equator. However, as can be seen from Figure 1.2, the present Earth's magnetic field significantly deviates from a pure axial dipole. In the first place, such deviations can be attributed to a tilt of about $11^{\circ}$ of the dipole component. Moreover, the fact that the magnetic poles are not antipodal (Figure 1.2, top right panel) reveals that non-dipolar contributions are also important.

The field intensity map of Figure 1.2 reveals further interesting insights. Axisymmetric lines of equal intensity are expected for a pure axial dipole with an intensity at the equator half of that at the poles. The geomagnetic field markedly differs from such configuration. Two features show that even a tilted dipole cannot account for the observed pattern. Firstly, the southern polar intensity maximum is somewhat stronger than the northern one, the latter also appearing as a double feature. Secondly, a strong minimum $(\sim 23 \mu \mathrm{T})$ localized in the South Atlantic, and for this reason known as the South Atlantic anomaly, is present.

Despite this complex morphology, the power of the geomagnetic surface field measured for each spatial degree $\ell$ as (Lowes 1974)

$$
R_{\ell}=\left(\frac{a}{r}\right)^{2 \ell+4} \sum_{m=0}^{\ell}\left[\left(g_{\ell}^{m}\right)^{2}+\left(h_{\ell}^{m}\right)^{2}\right]
$$

is dominated for more than $90 \%$ of its total amplitude by the dipole $\ell=1$ (see, e.g., Kono 2007). The second strongest contribution, lower by more than one order of magnitude in power, is the quadrupole $(\ell=2)$. The dipole approximation therefore reflects the dominant energetic contribution to the field. Furthermore, since the non-dipole terms vary on time scales shorter than the dipole, the geomagnetic field can be regarded as dipolar when averaged over a sufficiently long time. As will be seen in the following section, this assumption is often used in the study of the long-term geomagnetic field variations.

\subsection{The paleomagnetic field}

Systematic measurements of declination, inclination and intensity of the Earth's magnetic field are available only from the mid-19th century and have been recorded at observatories mainly located in Europe. The past geomagnetic field, however, has been preserved by the natural magnetization of rocks and sediments. Unfortunately, the quality of such data is often poor and therefore the experimental determination of direction and intensity results very difficult and sometimes poorly constrained.

Paleomagnetism is the study of the past geomagnetic (paleomagnetic) field as recorded by rocks, sediments and archaeological materials. Such studies are of fundamental importance because they can provide information on the history of magnetic field generation in the Earth's core. In Section 1.4.1 we give a general description of the physical mechanisms involved in the magnetization of rocks and sediments. Observational evidences of geomagnetic field reversals are summarized in Section 1.4.2. The characteristics of polarity transitions are described in Section 1.4.3, Our discussion on the paleomagnetic field 
concludes with a description of the most recent geomagnetic polarity time scale (Section 1.4.4) and of previous statistical analyses performed on different reversal chronologies.

\subsubsection{Rock magnetism}

In paleomagnetism, the remanent magnetization (RM) acquired under natural conditions by magnetic minerals is referred to as the natural remanent magnetization (NRM). The NRM of a rock is shaped by various magnetization phases which occurred during its geological history. Any rock, at the time of its formation, tends to acquire a magnetization $\mathbf{M}$ generally parallel to the ambient magnetic field $\mathbf{H}$. This is referred to as the primary magnetization and gives information about the direction and intensity of, presumably, the Earth's magnetic field. Several geological changes subsequent to the rock formation, such as chemical alteration and relaxation effects, may affect the primary magnetization inducing further magnetizations. Secondary magnetizations have to be excluded in laboratory measurements to retrieve unbiased information about the geomagnetic field when the rock was formed or cooled below its Curie temperature.

Different primary magnetization mechanisms may occur depending on the actual type of magnetic mineral. In ferromagnets, the magnetic moments of neighboring atoms are parallel and have the same magnitude. Ferromagnetic materials retain indeed a spontaneous remanent magnetization $M_{r}$ in the absence of an external field $H$. The high magnetic susceptibility $\chi$ of ferromagnets originates a generally strong induced magnetization $M_{i} \sim \chi H$ which can thus be measured even for weak magnetic fields such as the Earth's one. Among common magnetic minerals, only iron and iron-nickel are ferromagnetic but they are rarely found on Earth.

Prevailing magnetic minerals on Earth are magnetite and hematite and they are said to be ferrimagnetic and antiferromagnetic materials respectively. In antiferromagnetism, adjacent magnetic moments are oppositely directed and, since they are created by identical numbers and species of ions, have equal intensities. In a zero field, an antiferromagnet has in principle no remanence because the spontaneous (oppositely directed) magnetizations cancel on larger spatial scales. Nevertheless, both sets of spins can be deflected in a strong external field thus originating an antiferromagnetic susceptibility $\chi_{a}$ which varies only weakly with temperature (Dunlop and Özdemir 2007). Hematite actually has a weak permanent magnetization which occurs only for certain crystal symmetries. Deviations of magnetic moments out of antiparallelism by a fraction of a degree are also sufficient to generate a weak transverse ferromagnetic moment.

In ferrimagnetic minerals adjacent magnetic moments have different intensities because they do not belong to the same type of atom (or ion). Magnetite, for example, is constituted by antiparallel magnetic sub-lattices with a different number of $\mathrm{Fe}^{2+}$ and $\mathrm{Fe}^{3+}$ ions. This imbalance may induce a net magnetization $M_{i}$ when placed in an external field. This magnetization is generally weaker than that of ferromagnetic iron where all atomic moments add, but is larger than that of hematite (Dunlop and Özdemir 2007).

Above a certain critical temperature (Curie temperature) the disordering effect of thermal energy overcomes the ordered structure of magnetic moments. Magnetite, for example, has a Curie temperature of about $580^{\circ} \mathrm{C}$. When an igneous rock cools from above the Curie temperature of its magnetic minerals in an external field $\mathbf{H}$ (such as the Earth's magnetic field), the torque exerted on the magnetic moments orients them in the direction 
of $\mathbf{H}$ thus originating a net magnetization $\mathbf{M}$ of the sample. This type of magnetization regards igneous rocks and is referred to as the thermoremanent magnetization (TRM).

Extrusive igneous rocks such as lava flows cool very rapidly in their environment relative to changes of the Earth's magnetic field and therefore they represent almost instantaneous recordings of the field. At this stage, several mechanisms may create undesired secondary magnetizations. For example, the cooled and solid outer surface of lava flows may be rotated by the hotter and fluid layers beneath it. The TRM direction may then no longer accurately reflect the geomagnetic field. To overcome this problem, the consistency of magnetic directions is usually checked in different regions of the lava flows.

Due to the episodic nature of volcanic activity, lava flows often provide a record of the Earth's magnetic field which is discontinuous in time. Intrusive igneous rocks may, potentially, offer a continuous record of the magnetic field. Unfortunately, the rate of chemical alteration of magnetic minerals in igneous rocks increases dramatically with temperature. Since intrusive igneous rocks cool significantly slower than lava flows do, the chemical processes undergone often render this record difficult to resolve.

Sedimentary rocks acquire a stable NRM called the detrital remanent magnetization (DRM). Small magnetic grains, while sinking and settling at the bottom of lakes or in marine environments, statistically align with the geomagnetic field. When the bottom gets compacted by the overlying layers, these grains lock into the place. Different geological and biological processes may cause the rotation or translation of the sedimentary grains thus inducing secondary magnetizations (post-DRM).

Despite these problems, some deep-sea cores provided excellent records of the reversal chronology but, because of the low sedimentation rates, only in some cases of reversal transitions themselves. On the other hand, terrestrial sedimentary cores and certain lake sediments with higher sedimentation rates unrevealed geomagnetic field variations over a few thousand to a few tens of thousand years (Merrill 1998, Chapter 3).

A detailed discussion of the different magnetization processes occurring in rocks and the experimental measurement of their RM can be found in Dunlop and Özdemir (2007). An introduction to rock magnetism with particular emphasis to the determination of paleomagnetic directions and intensity is given by Merrill (1998, Chapter 3).

\subsubsection{Observational evidences for field reversals}

One of the most well documented observations of the paleomagnetic field are reversals. David (1904) and Brunhes (1906) first claimed to have observed magnetizations in lava flows roughly opposite to the present Earth's magnetic field direction. More than two decades later, Matuyama (1929) compiled the first, very approximative reversal chronology demonstrating that early Quaternary lavas had a reverse TRM. These studies, however, did not provide definitive evidence for reversals of the geomagnetic field.

An alternative explanation, at that time strongly supported in the scientific community, considered certain self-reversal mechanisms occurring in rocks (Néel 1951) as responsible for the reverse TRM observed. We now briefly describe the most common mechanism of self-reversal of thermoremanent magnetization. Consider a rock with two separated magnetic phases such that phase $\mathrm{A}$ has a higher Curie temperature than phase $\mathrm{B}$, namely $T_{C}^{(A)}>T_{C}^{(B)}$. When the rock cools to a temperature $T$ such that $T_{C}^{(B)}<T<T_{C}^{(A)}$, phase A is magnetized parallel to the external field $\mathbf{H}$. On further cooling below $T_{C}^{(B)}$, phase B 
becomes magnetized in the total field $\mathbf{H}+\mathbf{H}^{(A)}$, where $\mathbf{H}^{(A)}$ is the magnetic field due to phase A. The direction of the total field in which B becomes magnetized depends on the relative intensity of $\mathbf{H}$ and $\mathbf{H}^{(A)}$. If $\left|\mathbf{H}^{(A)}\right|>|\mathbf{H}|$, phase B will be magnetized opposite to $\mathbf{H}$. In particular, if $\mathrm{B}$ has a higher saturation magnetization at room temperature than $\mathrm{A}$, the sample will have self-reversed.

The controversy between field reversals and self-reversals in rocks continued for several years until the global character of the reverse field directions observed was firmly established. Cox and Doell (1963) and McDougall and Tarling (1963), using precise radiometric age determination on lava flows younger than $4 \mathrm{Ma}$, indeed demonstrated that reversals occurred at the same time at widely separated locations such as Alaska, California, Iceland and Hawaii. Secondary magnetization effects possibly affecting the measurements were also ruled out because simultaneous zones of one polarity were observed in rocks of widely different magnetic mineralogy (see, e.g., Wilson 1962). It should be noted that these early studies were not carried on continuous rock sequences but their main aim was to establish the global character of these events. A discussion on the developments and properties of a continuous reversal chronology will be the subject of Section 1.4.4.

Further observations of continuous field polarity changes carried on rock sequences (see Section 1.4.3) definitely ruled out the hypothesis of self-reversals and finally proved that the geomagnetic field reversed in the past. Today we have evidences of dipole field polarity changes as old as about $2.8 \mathrm{Ga}$ and several hundreds of these events have been recorded with a certain degree of reliability for the past $170 \mathrm{Myr}(\mathrm{Ogg}$ 2012). Properties of the field during such transitions are described in more detail in the following section.

\subsubsection{Geomagnetic polarity transitions}

The Earth's magnetic field has a complex morphology and varies on a wide range of time scales. Defining a reversal simply as a quasi-instantaneous sign change of the field thus falls too short. When averaged over few thousand years, however, the geomagnetic field is closely approximated by a geocentric axial dipole (Merrill 1998). Recent global field models (see, e.g., Pavón-Carrasco et al. 2014) show that the geomagnetic field can be averaged as axial dipolar in around $2000 \mathrm{yr}$ within $5^{\circ}$ confidence in latitude. Since such approximation is used in many paleomagnetic studies, a necessary requirement for a field reversal is that the axial dipole term $g_{1}^{0}$ in the spherical harmonic decomposition (1.3) has to change sign. This definition, however, is not sufficient. Paleomagnetic measurements are, indeed, often available only at few locations over the Earth's globe and cannot provide a valid spherical harmonic description of the field, especially throughout a complete polarity transition. Moreover, most records from igneous rocks seldom capture polarity transitions and uncertainties inherent to radiometric dating techniques of $1-2 \%$ are at least comparable to the event duration (Lowrie 2007, Chapter 5). Sedimentary records with high deposition rates of the order of $10 \mathrm{~cm} / \mathrm{kyr}$ are thus regarded as the most reliable recording media for polarity transitions but have the problem of averaging field variations over the (generally unknown) lock-in time. To overcome these difficulties, it is common practice in paleomagnetism to obtain, firstly, reliable transitional data at a single selected location.

The paleofield direction at the given location is typically characterized by the so-called virtual geomagnetic pole (VGP). The VGP position is where the pole of a geocentric 

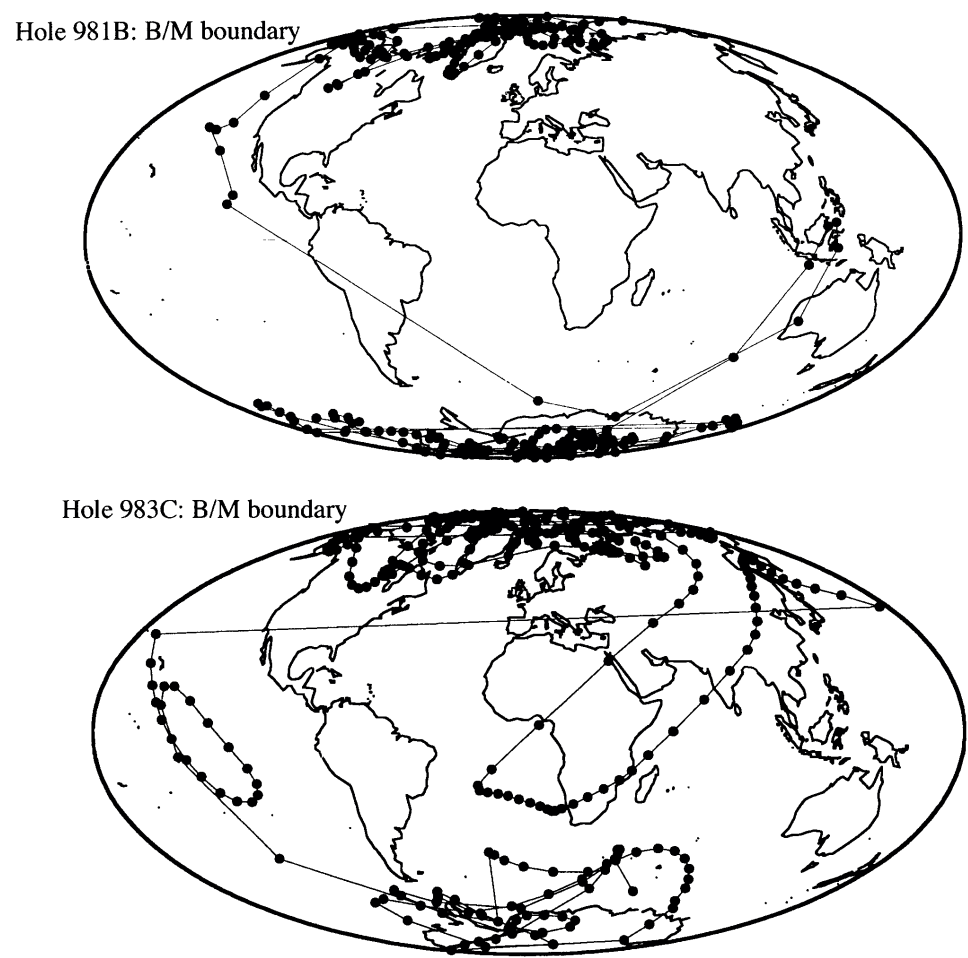

Figure 1.3: Virtual geomagnetic pole paths for the Brunhes/Matuyama polarity transition (from reverse to normal) from the Ocean Drilling Program Sites 981 (top panel) and 983 (bottom panel). Adapted from Channell and Lehman (1997).

dipole would be to give the observed field direction at the given location. Given the observed declination $D$ and inclination $I$ of the remanent magnetization measured in a sample, the VGP calculation is quite straightforward (see, e.g., Lowrie 2007, Chapter 5). This quantity is useful for comparing observed directions from different positions on the globe. It is important to note that the geocentric dipole assumption may fail if the field locally hosts significant multipolar contributions.

Examples of some high-quality VGP paths of the most recent Brunhes/Matuyama (B/M) transition, sampled from deep-sea sediment cores at two different sites (Ocean Drilling Program Sites 981 and 983), are presented in Figure 1.3, The B/M transition shows complex VGP paths with considerable scatter around the geographic poles. The deposition rates at Site 983 are about twice those at Site 981 as it is evident from the finer temporal variability recorded by the former core. As a consequence of the large directional changes experienced by the dipole field during polarity transitions, reversal durations are difficult to estimate and mainly suffer from the arbitrary definition of transitional VGPs. It is a common practice in paleomagnetism to regard VGPs as transitional when deviating more than $45^{\circ}-60^{\circ}$ from the closest geographic pole (Merrill and McFadden 1999).

The apparent duration of reversals varies between different localities and the $\mathrm{B} / \mathrm{M}$ transition represents one of the most well documented examples (Dormy et al. 2000). If the non-dipole field components, fluctuating on shorter time scales, locally dominate in intensity relative to the dipole, the duration of directional changes can be vastly diverse at different sites. The duration of a polarity reversal is, however, likely bounded by 1 and 8 


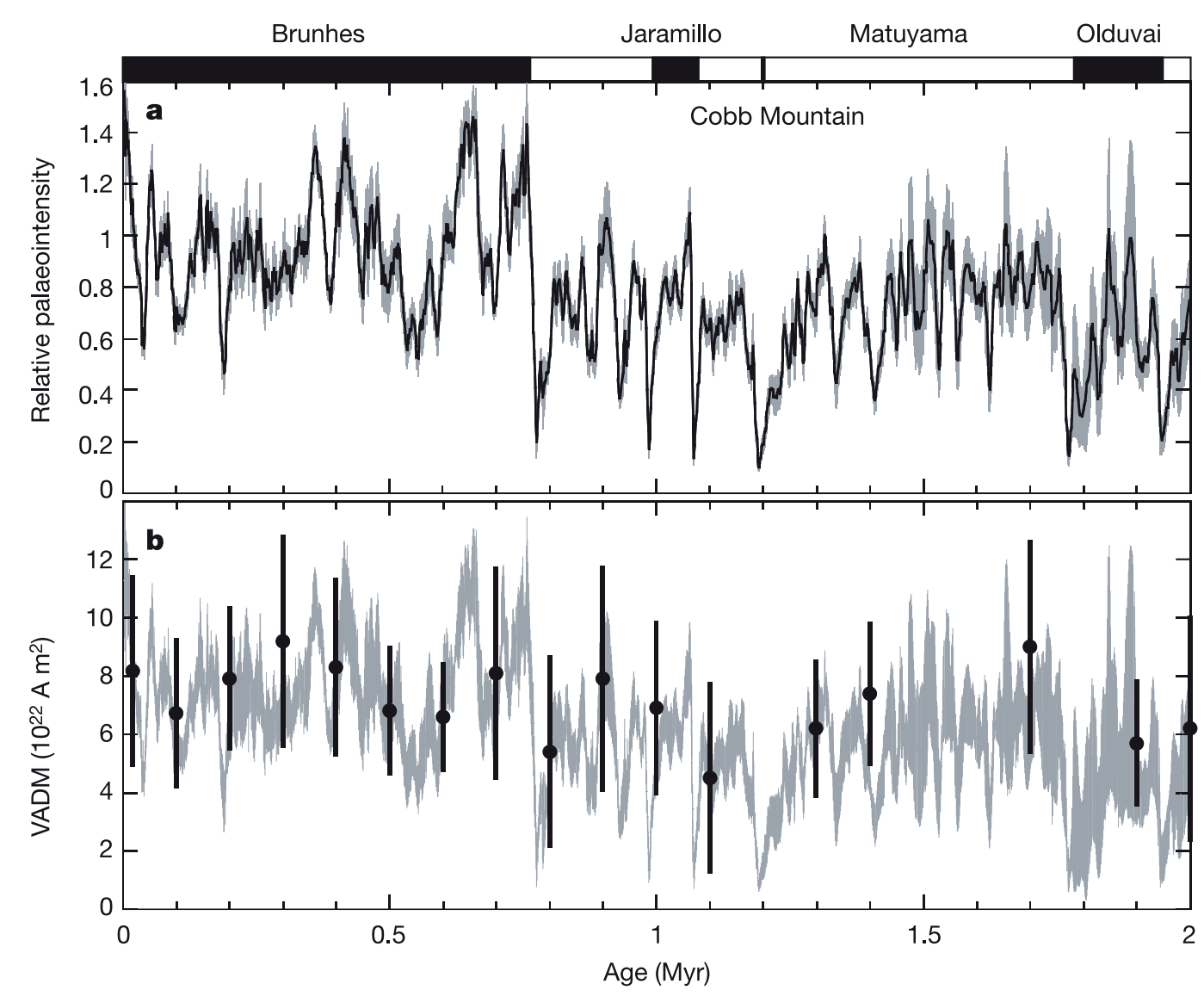

Figure 1.4: Evolution of the geomagnetic field intensity during the past $2 \mathrm{Myr}$. Panel a: Composite relative paleointensity curve (95\% confidence intervals in gray). Black and white bars at the top of the panel depict normal and reverse field polarities respectively. Panel $b$ : Virtual axial dipole moment (VADM) obtained after calibration with the absolute paleointensity from volcanic records. Black dots denote VADMs from volcanic records averaged over successive time intervals (at least $0.1 \mathrm{Myr}$ long) and error bars indicate their dispersion. Adapted from Valet et al. (2005).

thousand years with a mean value of 4 - 5 thousand years (Merrill and McFadden 1999).

A further reasonable requirement for a proper definition of the event "reversal" is that the polarity change exhibits some stability after it occurred. Common paleomagnetic practice thus characterizes a reversal as a globally observed polarity change in the dipole field (mainly inferred from VGP measurements as discussed above) averaged over a few thousand years (Merrill 1998). In other words, this definition requires that the field presents a clear geocentric dipole character over a certain period of time bounding the transition. In this work, we used this property as a guideline for the identification of reversals in numerical dynamo simulations (see Section 5.3).

The past geomagnetic field intensity (paleointensity) is the second source of information on the characteristics of polarity transitions. Unfortunately, reliable paleointensity estimates are more difficult to obtain than directional data. Absolute paleointensity estimates are retrieved from igneous rocks and rely on laboratory measurements of their TRM, while relative estimates can be occasionally acquired from sediments (Merrill and McFadden 
1999).

Similarly to the analysis of directional data, it is convenient to introduce a reference measure for comparing paleointensity estimates obtained at different locations. The virtual dipole moment (VDM) is defined as the equivalent (geocentric) dipole moment leading to the observed field intensity and inclination in a rock sample. Figure 1.4 shows the evolution of the geomagnetic dipole field intensity during the last $2 \mathrm{Myr}$ as reconstructed by Valet et al. (2005). The relative paleointensity curve (Figure 1.4 panel a) has been obtained by stacking independent sediment cores records from different areas of the world. Note that polarity transitions correlate remarkably well with local minima of the field intensity.

The relative paleointensity curve has been calibrated with volcanic records to obtain an absolute estimate of the virtual axial dipole moment (VADM, panel b of Figure 1.4). This procedure, common to many paleomagnetic studies, is delicate and requires particular care. Remanent magnetizations of igneous rocks represent almost instantaneous records of the total field and thus provide information on both dipolar and non-dipolar field contributions. In order to eliminate eventual non-dipolar contributions to the estimated field intensity, Valet et al. (2005) used the time-averaged VADM recorded by lava flows over the past $0.8 \mathrm{Myr}$ for calibration. VADMs from volcanic records averaged over successive time intervals (denoted by black dots in Figure 1.4) agree with the calibrated record. The mean VADM value during the Brunhes polarity interval $(0-780 \mathrm{ka})$ is $(7.5 \pm 1.7) \times 10^{22} \mathrm{Am}^{2}$ and VADMs typically reach values below $20 \%$ of the mean during each polarity transition. It is important to remark that the non-dipolar field components may occasionally become comparable in intensity to the dipole during polarity transitions. Therefore, VDMs are not fully reliable estimates of the dipole field during transitional periods.

The dipole intensity decrease during polarity transitions has been confirmed by several paleomagnetic studies. Tanaka et al. (1995), for example, showed that significantly low VDM values occur when the VGP latitude is more than $45^{\circ}$ away from the closest geographic pole for several polarity transitions during the past $10 \mathrm{Myr}$. Furthermore, field intensity variations are generally observed to last longer than directional changes (Dormy et al. 2000). Large episodes of dipole intensity decrease are indeed necessary to cause a dominance of non-dipolar contributions, thus allowing for significant angular deviations of the magnetic pole. For a complete review of both directional and intensity observations during polarity transitions and their implications on the nature of the field we refer to Merrill and McFadden (1999).

Once the local characteristics of a transitional field are uncovered by the paleomagnetic measurements discussed above, the global character of a reversal has still to be determined. As already discussed in Section 1.4.2, this is typically done combining measurements from rocks of the same age at different sites over the globe. This usually represents enough evidence for a sign change of $g_{1}^{0}$ in the field expansion (1.3).

Transitional VGPs show a second type of events known as geomagnetic excursions. During excursions the magnetic pole greatly departs from the geocentric axial dipole direction. Such deviations are generally larger than typical paleosecular variations and may eventually lead to the reverse direction for a relatively brief period. Contrary to reversals, the opposite dipole direction is not firmly established during excursions. Brief polarity excursions were initially considered either as spurious and isolated recording artifacts indicative of remagnetization processes, or as local anomalies of the geomagnetic field. 
The analysis of high resolution deep-sea sedimentary records demonstrated that several excursions punctuated the most recent stable polarity epochs. Once the same excursions could be detected at several locations and in different magnetic materials, thus robustly establishing the global character of some of these events, their existence could no longer be doubted.

Numerous field excursions have been discovered to punctuate the most recent Brunhes polarity interval $(0-780 \mathrm{ka})$. The Laschamp excursion is the most well documented event and also the first to be historically recognized in lavas from the French Massif Central. Absolute ages of lava flows date its occurrence at $46.6 \pm 2.4 \mathrm{ka}$ and sedimentary records estimate its duration to about $2 \mathrm{kyr}$ (Dormy et al. 2000). Six excursions during the Brunhes polarity epoch can be reliably considered as global events, while at least five

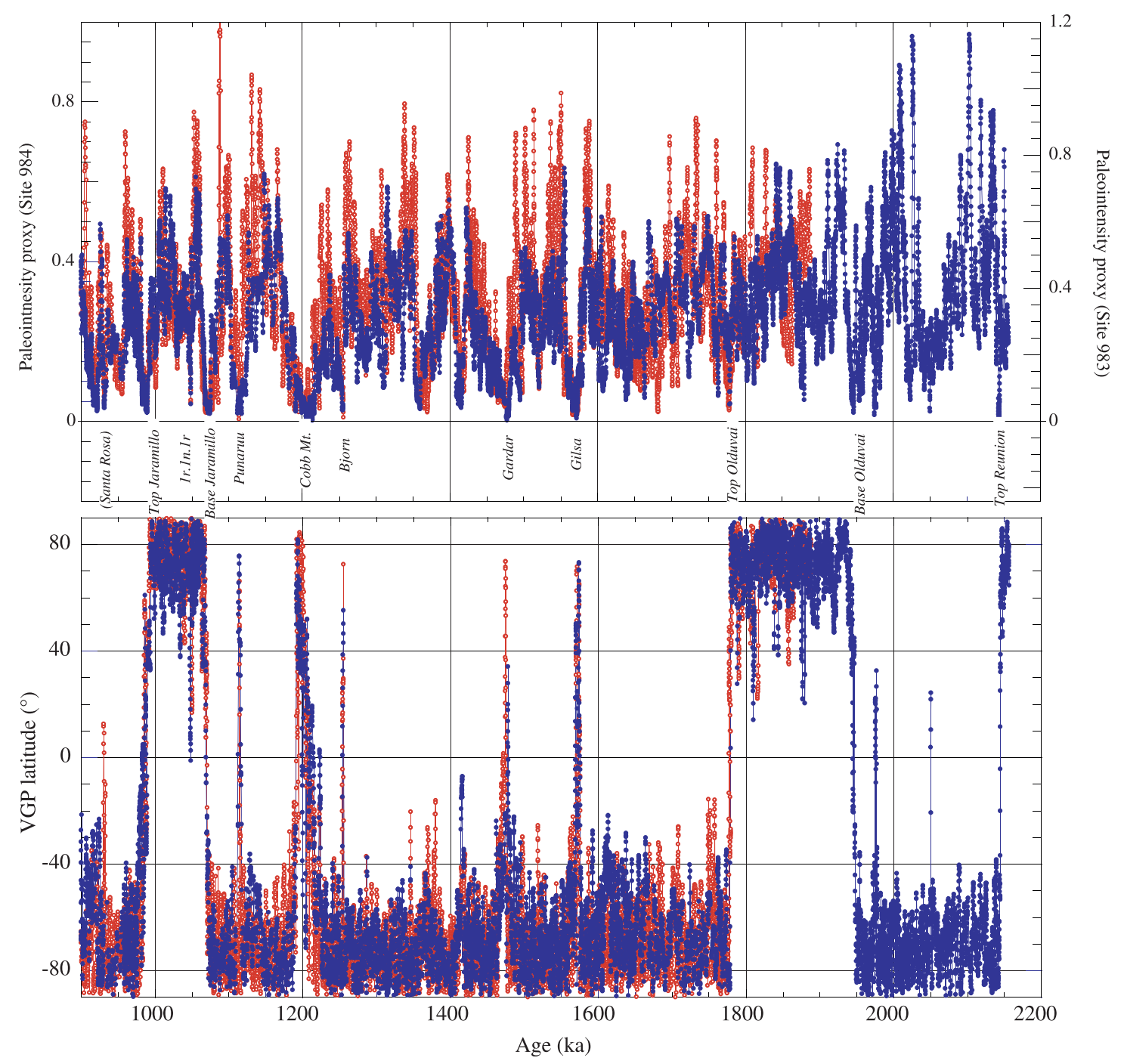

Figure 1.5: Relative paleointensity (top panel) and virtual geomagnetic pole (VGP) latitude (bottom panel) at the Ocean Drilling Program Sites 983 (red curves) and 984 (blue curves) during part of the Matuyama chron $(0.780-2.581 \mathrm{Ma})$. Reversals and globally correlated excursions are marked with the respective names. Adapted from Channell et al. (2002). 
more have not been correlated worldwide.

Figure 1.5 shows relative paleointensity estimates and VGP latitudes during part of the most recent reverse polarity interval (Matuyama chron, $0.780-2.581 \mathrm{Ma}$ ) as recorded from deep-sea sediments at the Ocean Drilling Program Sites 983 (in red) and 984 (in blue). Large fluctuations in the VGP latitude during the reverse polarity epoch between 1100 and $1780 \mathrm{ka}$ cross the (virtual) magnetic equator and characterize geomagnetic excursions, named with the locality of their discovery. Jaramillo and Olduvai reversals embed shorter epochs of normal polarity (positive VGPs) and do not present any excursion. A comparison with the paleointensity records (Figure 1.5, top panel) reveals a correlation between excursions and local field intensity minima. As demonstrated by Guyodo and Valet (1999), the six global excursions identified during the most recent Brunhes normal polarity epoch also nicely correlate with major paleointensity lows. Further information regarding dating and duration of excursions during the Brunhes and Matuyama polarity epochs can be found in, e.g., Laj and Channell (2007) and Valet et al. (2008). Evidences for geomagnetic excursions in periods older than the Matuyama are more difficult to access because the respective transitions need to be entirely resolved to determine an event occurrence.

On the basis of the evidences described above, it is likely that excursions punctuated the entire history of the Earth's magnetic field. Since excursions represent a major characteristic of the geomagnetic field, their properties must be considered as an actual constraint for dynamo models. In order to classify an event as a field "excursion", it appears crucial to distinguish it from: (i) a large secular variation period and (ii) a pair of subsequent reversals (Gubbins 1999). Such distinction appears also fundamental in numerical dynamo simulations when these events have to be properly identified. This issue will be discussed in detail in Section 5.3 .

\subsubsection{The geomagnetic polarity time scale}

The first, very crude geomagnetic polarity time scale (GPTS) was compiled by Cox and Doell (1963) and comprised only three magnetic polarity epochs for the past 3.2 Myr with a duration of $\sim 1 \mathrm{Myr}$ each. When new observations and refined dating techniques became available, more reversals started to be included in this early GPTS. In particular, polarity epochs shorter than one hundred thousand years were discovered to punctuate the longer ones. Figure 1.6 presents the GPTS for the past $6 \mathrm{Myr}$ as compiled by Cande and Kent (1995). Longer polarity epochs are named in honor of pioneering geophysicists (Brunhes, Matuyama, Gauss, Gilbert, etc.), while shorter events are labeled with the location of their discovery. A nomenclature of polarity epochs based on their duration has been officially adopted and is summarized in Table 1.1. Polarity epochs with a duration of $1-10 \mathrm{Myr}$ are called chrons, while events lasting 0.1-1 Myr are referred to as subchrons. We currently live during the Brunhes chron of normal (N) field polarity. The last reversal occurred about $780 \mathrm{kyr}$ ago and the most recent reverse $(\mathrm{R})$ polarity chron (Matuyama) is punctuated by three subchrons (Jaramillo, Olduvai, Réunion as also shown in Figure 1.5). Very brief polarity intervals, typically shorter than $30 \mathrm{kyr}$ and which are not fully constrained, are termed cryptochrons. During the actual polarity epoch a cryptochron occurred, for example, $0.504 \mathrm{Myr}$ ago (Cobb Mountain). A list of cryptochrons for the last $64 \mathrm{Myr}$ can be found in Table 3 of Cande and Kent (1995). It is important to remark that 


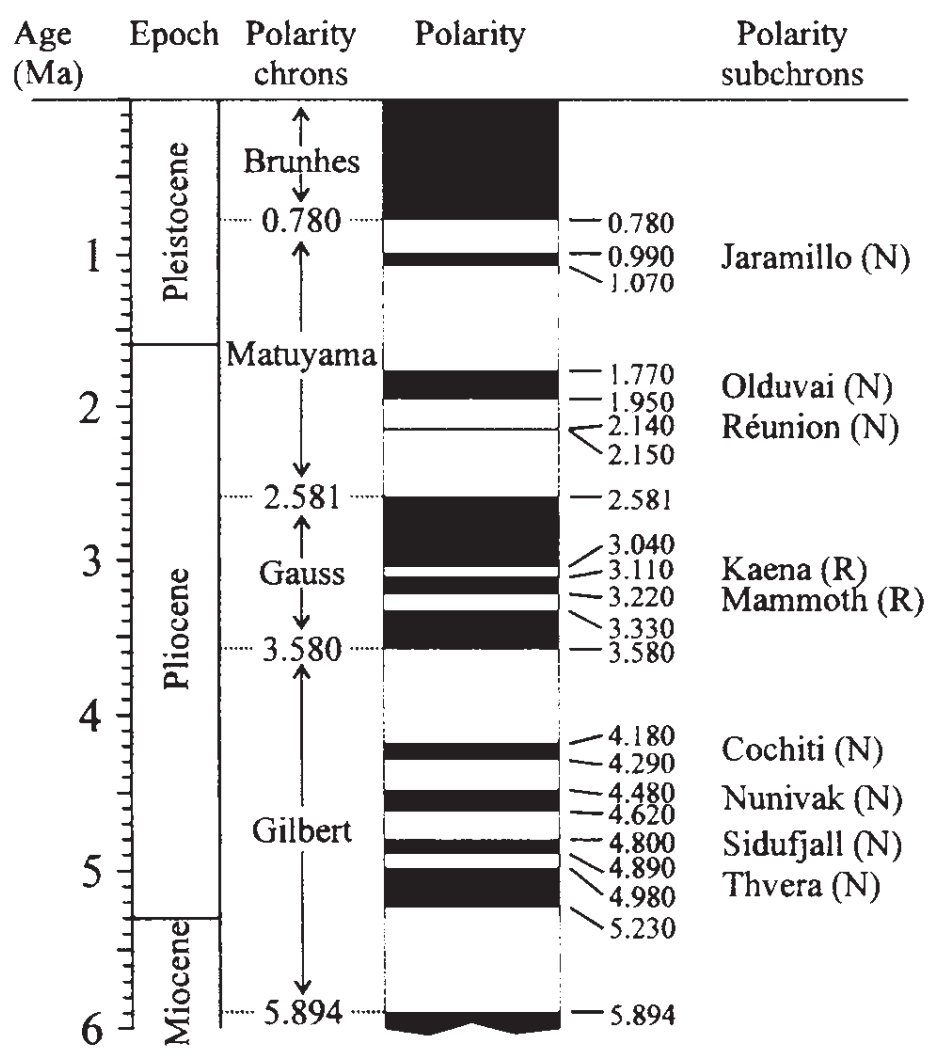

Figure 1.6: Geomagnetic polarity time scale of Cande and Kent (1995) for the past 6 Myr. Normal and reverse polarities are marked in black and white respectively. From Merrill (1998).

geomagnetic excursions, having typical durations of few thousand years, are sometimes difficult to distinguish from cryptochrons.

A continuous record of polarity changes can be obtained analyzing the paleomagnetic field recorded in deep-sea sediments retrieved by drilling the ocean bottom. Typical sedimentation rates of the north-central Pacific Ocean are relatively low and of the order of 1 to $10 \mathrm{~mm}$ per thousand years (Merrill 1998). Such low sedimentation rates allow to go

\begin{tabular}{ccc}
\hline $\begin{array}{c}\text { Magnetostratigraphic } \\
\text { polarity units }\end{array}$ & $\begin{array}{c}\text { Geochronologic } \\
\text { unit (time equivalent) }\end{array}$ & $\begin{array}{c}\text { Approximate } \\
\text { duration [yr] }\end{array}$ \\
\hline Polarity megazone & Megachron & $10^{8}-10^{9}$ \\
Polarity superzone & Superchron & $10^{7}-10^{8}$ \\
Polarity zone & Chron & $10^{6}-10^{7}$ \\
Polarity subzone & Subchron & $10^{5}-10^{6}$ \\
Polarity cryptozone & Cryptochron & $<3 \cdot 10^{4}$ \\
\hline
\end{tabular}

Table 1.1: Nomenclature for magnetostratigraphic (rock) and polarity chron (time) units as recommended by International Subcommission on Stratigraphic Classification. Adapted from $\mathrm{Ogg}(2012)$. 


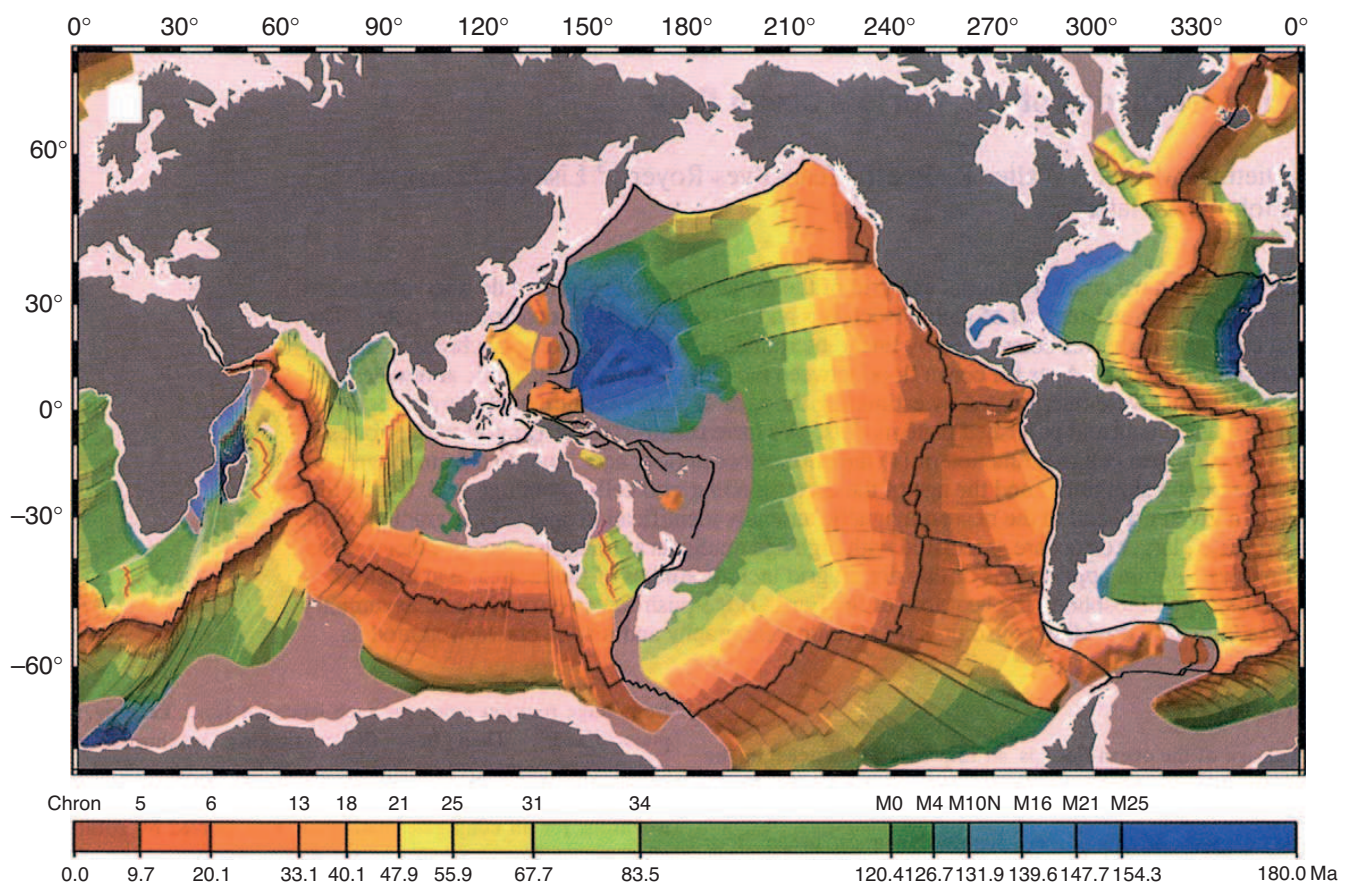

Figure 1.7: Digital isochrons of oceans based on magnetic chronology. Mid-ocean ridges are marked by the black solid lines. Adapted from Kono (2007).

back in time for several million years in the geomagnetic history over relatively short core lengths. Large uncertainties in the marine sediment record come from dating fossils and modelling variations in sedimentation rates. Accurate observations extending more than $10 \mathrm{Myr}$ ago are thus practically impossible in deep-sea sediments. Long sequences of lava flows on land are also affected by errors in their radiometric dating. Since these errors increase proportionally to the sample age, many of these extended records are useless.

A fundamental improvement in the compilation of a GPTS, now reliably extended to about $160 \mathrm{Myr}$ ago, came from Vine and Matthews (1963) who interpreted the magnetic anomalies observed at oceanic ridges. Mid-ocean ridges constitute geologically active fractures of the crust where hot magma, coming from mantle upwellings, constantly emerges at the ocean floor (see Figure 1.7). The extrusion of new material pushes the cooling crust away from the ridge symmetrically on either side. The large scale motion of tectonic plates, sinking in the mantle at oceanic trenches, generates tension at the ridge axis which tends to pull the crust and the uppermost mantle apart. Spreading rates range from about $20 \mathrm{~km} / \mathrm{Myr}$ to more than $140 \mathrm{~km} / \mathrm{Myr}$ (McElhinny and McFadden 2000). As the iron-rich material extruded at the ridge axis cools below its Curie temperature it becomes magnetized parallel to the Earth's magnetic field. The oceanic crust therefore acquires a normal or reverse remanent magnetization depending on the ambient field polarity at that time. Due to the sea-floor spreading mechanism described above, strips of alternating polarity (produced parallel and distributed symmetrically to the ridge axis) constitute the past geomagnetic polarity epochs (Figure 1.7). In other words, the crust near the oceanic ridges acts as a kind of "tape-recorder" of the Earth's magnetic field in the past. The field polarities are observed by magnetometers dragged over the ocean floors as anomalous field intensities. An enhanced magnetic field intensity is recorded 

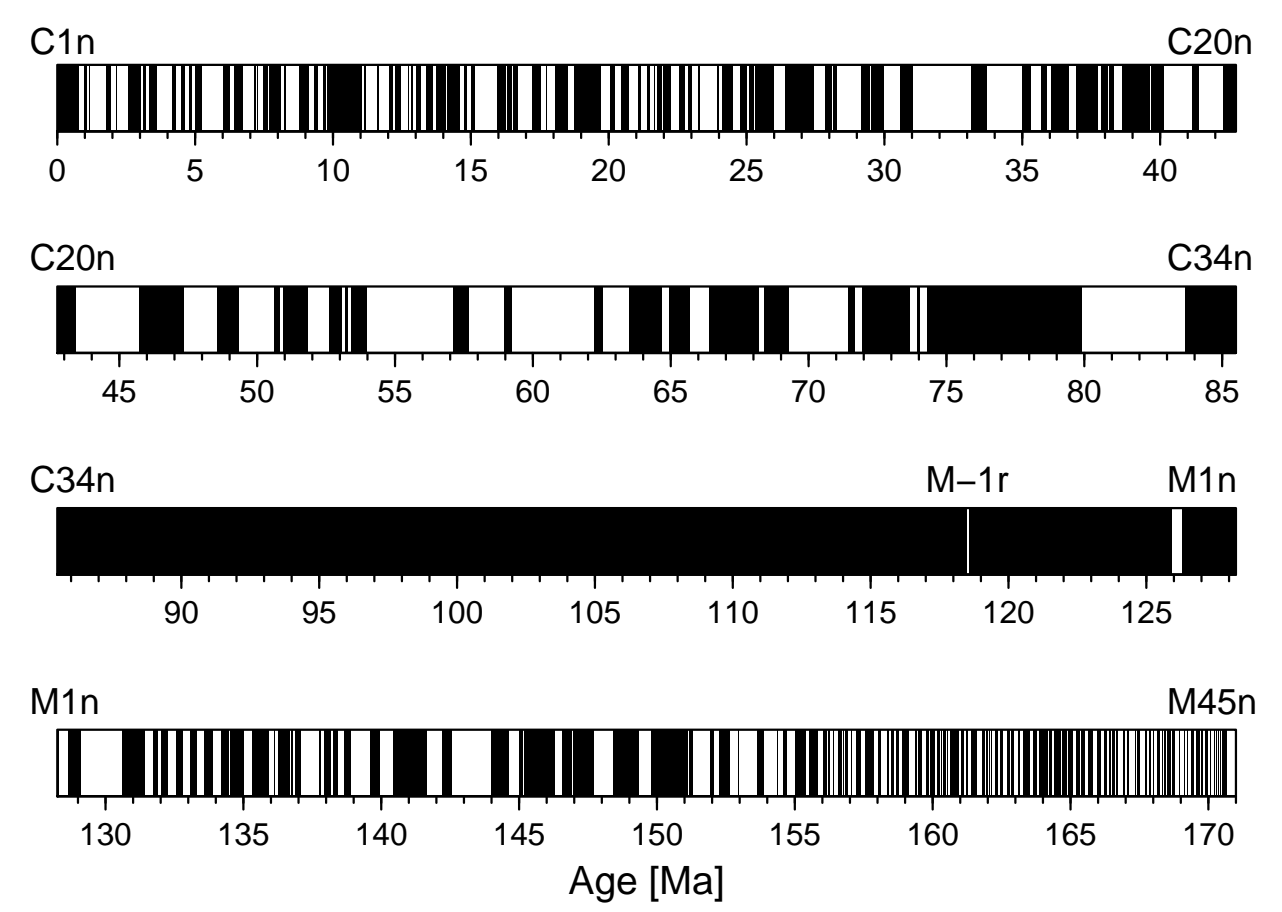

Figure 1.8: Geomagnetic polarity time scale of Ogg (2012) for the past $171 \mathrm{Myr}$. Black (white) marks normal (reverse) field polarity.

over the crust that cooled in an ambient field with the same polarity as the present field. Conversely, partial cancellation of the signal is observed over the crust formed during a period of reverse polarity.

The same sequence of magnetic anomalies is observed in much of the Pacific, Atlantic and Indian Oceans at the respective ridge axes. The South Atlantic magnetic anomaly profile has been taken as a standard for the polarity sequence spanning the last $84 \mathrm{Myr}$. Since this period covers the late Cretaceous through the Cenozoic, the associated reversal chronology is generally referred to as the $C$-sequence and the respective magnetic anomalies have been numbered from 1 to 34 (oldest). Polarity chrons of the $\mathrm{C}$-sequence are usually denoted by the letter " $C$ " followed by a number correlated with the youngest magnetic anomaly. A suffix " $n$ " ("r") marks the normal (reverse) field polarity during that period. For example, C1r denotes the Matuyama chron representing the period of reverse polarity between magnetic anomalies 1 and 2 . If a polarity chron contains shorter field polarities, the subchrons are denoted with a corresponding numbered suffix. For example, C1r.1n represents the youngest normal subchron during the Matuyama chron (Jaramillo event in Figure 1.5).

Figure 1.8 shows the most recent GPTS for the past $171 \mathrm{Myr}$ as reported by $\mathrm{Ogg}$ (2012). This reversal chronology contains 449 events which seem to occur stochastically in time. An exceptionally long normal polarity interval of $35 \mathrm{Myr}$ (chron C34n), known as the Cretaceous normal superchron (CNS), extends from about $118 \mathrm{Ma}$ to approximately $83 \mathrm{Ma}$. Such event is not unique in the past geomagnetic history. Evidences for a superchron of reverse polarity, known as the Kiaman reverse superchron (KRS), were already available before the reversal chronology was well developed. The KRS has been estimated to extend for more than $50 \mathrm{Myr}$, from approximately $318 \mathrm{Ma}$ to $262 \mathrm{Ma}$ 
(Cottrell et al.2008). Several magnetostratigraphic observations support its existence, but this event is too old to be recorded by marine magnetic anomalies.

A third superchron, known as the Moyero reverse superchron (MRS), is suspected to have occurred during the Ordovician from $485 \mathrm{Ma}$ to $463 \mathrm{Ma}$. Since evidences have been found only at a single site (Moyero, Siberia) and different records elsewhere do not document its existence, the MRS occurrence is still debated (McElhinny and McFadden 2000).

Oceanic crust of the late Middle Jurassic through the Early Cretaceous displays a second series of magnetic anomalies, named the M-sequence because they cover the Mesozoic era. The M-sequence comprises chrons M0r-M25r and can be considered quite robust since it has undergone only minor refinements since its first derivation ( $\operatorname{Ogg} 2012)$. Note that the end of M0r does not define the staring time of the CNS (Figure 1.8). After the M-sequence was numbered, clusters of brief reverse polarities were reported by deep-sea sediments from the oldest portion of the CNS. An upward continuation of the M-sequence suggested a negative numbering (M-1r) to define the brief chron which ended 118.5 Myr ago and which actually defines the start of the CNS. Despite this event has not yet been fully verified, we included it in the GPTS of Figure 1.8 because it is reported in other polarity time scales as well (see, e.g., Cande and Kent 1995). Two additional events occurred around $102 \mathrm{Ma}$ and $108 \mathrm{Ma}$ are not included in Figure 1.8 since their durations are not known.

Additional marine magnetic surveys in the Pacific using magnetometers towed near to the sea-floor allowed to extend the M-sequence up to about $171 \mathrm{Myr}$ ago (see Ogg 2012, and the oldest numbered M-sequence anomaly M45n). Contrary to surface surveys, these intensity observations just above the oceanic crust inevitably record short-wavelength features. It is therefore important to keep in mind that certain brief chrons might be an artifact of the recording method employed in the oldest $14 \mathrm{Myr}$ of this extended M-sequence.

\subsubsection{Statistical analyses of geomagnetic reversal occurrences}

In the attempt to provide robust constraints on our knowledge of the internal core processes underlying the occurrence of reversals, great effort has been spent in the statistical study of the GPTS since its earliest compilations. Previous statistical studies of geomagnetic reversal chronologies are reviewed in this section. Predictions from reversing geodynamo models are also discussed in the following.

As already described in Section 1.4.3, paleomagnetic observations indicate that the time required for a polarity transition typically ranges from 4 to $5 \mathrm{kyr}$. This time is more than two orders of magnitude shorter than the mean chron duration during the Cenozoic. As a first approximation, it is therefore reasonable to consider reversals as rare events occurring instantaneously in time. The further assumption that reversals are independent events (i.e. the probability of an event occurrence does not depend on the duration of prior polarity intervals) has been claimed in the pioneering work of Cox (1968) but was subsequently debated (see, e.g., Naidu 1974).

The first and most obvious reason which may break the independence assumption is that reversals, although relatively brief, do not occur instantaneously and therefore a certain degree of memory must be present in the magnetic field. If fluid motions powering the geodynamo were to terminate, the dipole field would decay by ohmic diffusion on 


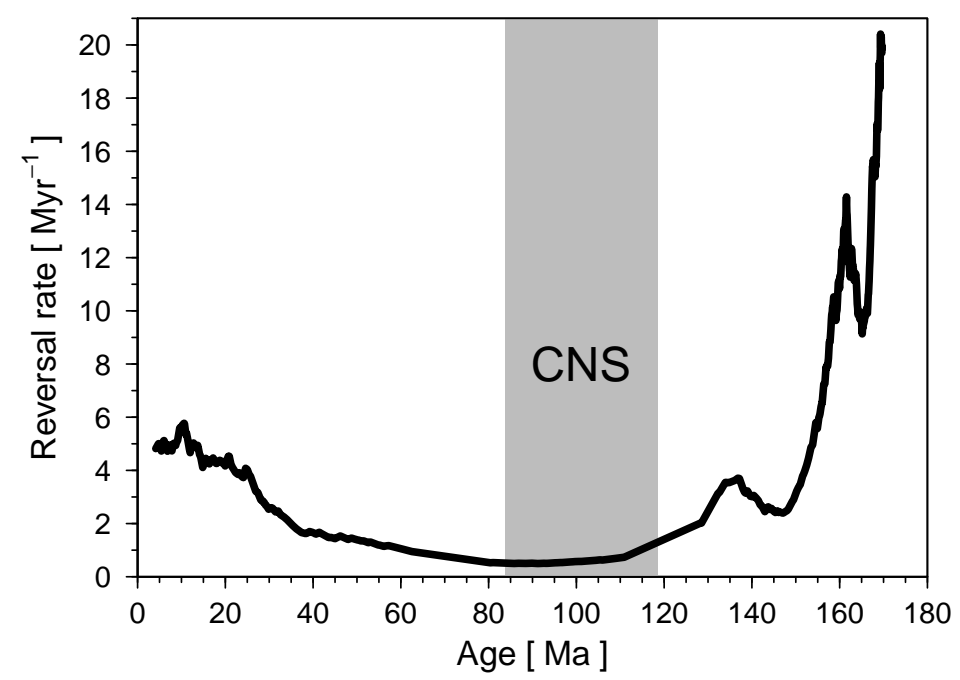

Figure 1.9: Reversal rate for the past $170 \mathrm{Myr}$ according to the time scale of $\mathrm{Ogg}(2012)$. The estimate has been constructed using a sliding window with a fixed number of $N=40$ reversals in each interval. The duration of the Cretaceous normal superchron (CNS) is marked in gray.

time scales of the order of several tens of thousand years (see Section 1.2). The free dipole decay time of the core $(\sim 56 \mathrm{kyr})$ can therefore be regarded as a reasonable upper bound for the memory time of core processes. On the other hand, the memory time might be several orders of magnitude shorter if it directly reflects typical temporal variations of internal fluid motions. The convective overturn-time, for example, amounts to a few hundred years and is associated with large scale motions of convection. Analyzing a suite of global numerical dynamo simulations, Hulot et al. (2010) suggested that the Earth's dynamo is totally unpredictable beyond a century. Later studies demonstrated that dipole correlation times in such models are typically a fraction of the free dipole decay time, thus suggesting that any departure from the independence of reversal occurrences might be small (Lhuillier et al. 2011, Lhuillier et al. 2013).

The second reason which might invalidate the independence assumption are the variations observed in the reversal rate. Figure 1.9 shows the reversal rate throughout the GPTS of Ogg (2012) calculated using a sliding window with a fixed number of reversals. The reversal rate decreases when approaching the CNS and increases afterwards, thus indicating the possible existence of statistical correlations between successive field polarities. However, since the reversal rate changes slowly compared to the mean chron duration (which amounts to about $0.38 \mathrm{Myr}$ ), deviations from independence might still be considered mild. Correlation analyses performed on short periods where the reversal rate can be considered stationary indeed support the hypothesis of statistical independence (Phillips et al. 1975, Phillips and Cox 1976).

In principle, the non-stationarity of the reversal rate over the last $170 \mathrm{Myr}$ precludes the possibility of considering the polarity chrons as a random sample from a probability distribution with fixed parameters. The structure of the non-stationarity has been included into the parameters of certain distribution functions either by assuming a filtering process of the shortest chrons (McFadden 1984) or by analyzing the reversal sequences in stationary sub-intervals (McFadden and Merrill 1984). These and further aspects regarding the 
characterization of the distribution of geomagnetic polarity intervals will be discussed in Section 3.4.1 where different probability distribution functions are described.

Constable (2000) investigated in detail the temporal variations in the reversal rate during the Cenozoic. The author demonstrated that the hypothesis of a non-stationary Poisson process is statistically compatible with the observed sequence of reversals.

Long-term variations in the reversal rate (and ultimately superchron states) have been successfully linked to external effects influencing the core field dynamics and most often identified to be of mantle origin (McFadden and Merrill 1984, Biggin et al. 2012). The typical time scale of convective motions in the mantle can be estimated as $\tau_{m}=D / U \approx$ $200 \mathrm{Myr}$, where $D$ is the mantle thickness and $U$ the mean sea-floor spreading rate of $45 \mathrm{~mm} / \mathrm{yr}$, and suggests that such processes and the occurrence of the CNS might have a causal link. In this context, Driscoll and Olson (2011) proposed a numerical dynamo model able to reproduce the reversal rate variations observed in the GPTS. The authors showed that an increase in the heat-flow at the core-mantle boundary drives the dynamo from a stable dipolar to a reversing state which accounts for reversal rate variations remarkably similar to those observed since the end of the CNS. Conversely, a monotonic decrease in the heat-flow at the outer boundary produces a decreasing reversal rate.

Other mechanisms possibly influencing the long-term state of the Earth's core have also been proposed. Driscoll and Olson (2009), for example, demonstrated that incremental changes in the convective forcing of a chemically driven dynamo started in a non-reversing state evolves to a reversing regime. The authors also showed that trends in dipole intensity and polarity interval durations in such models qualitatively agree with those obtained from the analysis of reversal chronologies since the end of the CNS.

However, it is not yet clear if external processes are necessarily required to reproduce the observed reversal rate variations. Such changes might alternatively stem from the highly non-linear internal processes governing the geodynamo and thus spontaneously producing the large variety of time scales observed. This alternative hypothesis was proposed, for example, by Gaffin (1989) who demonstrated that polarity chrons with durations longer than $0.5 \mathrm{Myr}$ are well described by a power-law distribution. The author interpreted the scale invariance of the power-law distribution as an evidence for the lack of an intrinsic time scale in the internal dynamical processes triggering reversals. More recently, Jonkers (2003) confirmed these results analyzing the Cenozoic reversal chronology of Cande and Kent (1995) but obtained a different power-law exponent.

Compared to other probability distributions describing paleomagnetic chron durations, the power-law presents a slower decreasing tail (see, e.g, Shcherbakov and Fabian 2012). This characteristic naturally accounts for the occurrence of extreme events such as superchrons. Rather than externally induced, these events might therefore alternatively stem from the same physical process leading to shorter polarity intervals. Hulot and Gallet (2003), generalizing a statistical test for stationarity introduced by McFadden and Merrill (2000), showed that there is no long-term behavior in the reversal rate over the $40 \mathrm{Myr}$ preceding the CNS that could have explained its onset. In support of these findings, Carbone et al. (2006) used a statistical transform to demonstrate that a non-stationary Poisson process is incompatible with different polarity time scales available for the Cenozoic. The authors furthermore showed that a Lévy process, a particular type of random walk characterized by a heavy-tailed distribution, well describes the statistic of paleomagnetic chron durations. A detailed comparison of different distribution functions 
which adequately model the paleomagnetic polarity intervals have been carried out by Ryan and Sarson (2007) and Shcherbakov and Fabian (2012). Such simple statistical characterizations of reversal occurrences are particularly intriguing because they may provide the link between complex geodynamo processes and simpler stochastic mechanisms able to describe them.

As a final remark, it is important to keep in mind that two unavoidable problems affect the analysis of paleomagnetic reversal chronologies. Firstly, a reasonably reliable GPTS is available only for the past $160 \mathrm{Myr}$ and this provides a relatively short record of polarity intervals. Secondly, due to resolution problems, even the most recent versions of the GPTS likely disregard several of the shortest chrons (typically shorter than $30 \mathrm{kyr}$ ). Furthermore, the precise ages of several reversals still need to be adjusted. Marzocchi (1997), for example, investigated the influence of this incompleteness on some statistical properties of the GPTS. Filtering the shortest polarity intervals from synthetic series of events, the author showed that the Cenozoic time scale of Cande and Kent (1995) is compatible with a Poisson process where at least $30 \%$ of the shortest chrons are missing in the record. As already pointed out in Section 1.4.4, two of such short, not fully verified events may have occurred during the CNS around $108 \mathrm{Ma}$ and $102 \mathrm{Ma}$ (Ogg 2012). The problems discussed above define an inherently incomplete GPTS and the statistical analysis of reversal chronologies always require careful interpretations. 



\section{The geodynamo: theory and numerical modelling}

This chapter introduces the fundamental magneto-hydrodynamic equations which model the geodynamo. The equation governing fluid motions under the Boussinesq approximation and a transport equation for the temperature perturbations are derived in Sections 2.1 2.4. while the equation of evolution for the magnetic field is derived in Section 2.5. The non-dimensionalization of the governing equations is discussed in Section 2.6. Section 2.7 discusses appropriate boundary conditions for the geodynamo problem. The chapter closes summarizing the numerical approach employed to solve the above-mentioned equations in Section 2.8 .

\subsection{Hydrodynamics}

The Earth's outer core is a liquid mixture of iron and nickel. Since we are interested in the description of macroscopic phenomena in such a system, namely occurring on length scales much larger than the typical interatomic distances, the liquid core can be regarded as a continuous medium. In this approximation, known as the continuum hypothesis, the state of the fluid is described by physical quantities which are continuous functions of position $\mathbf{x}$ and time $t$ (Eulerian specification).

The state of a moving fluid is completely determined by the distribution of its velocity $\mathbf{u}=\mathbf{u}(\mathbf{x}, t)$ and of any two thermodynamic quantities such as, for example, the pressure $P=P(\mathbf{x}, t)$ and the density $\rho=\rho(\mathbf{x}, t)$. All the other thermodynamic quantities can be obtained from the latter two specifying an equation of state. We now recall the equations governing fluid motions.

The conservation of mass of the fluid is described by the equation of continuity

$$
\frac{\partial \rho}{\partial t}+\nabla \cdot \rho \mathbf{u}=0
$$

which can be written, expanding the divergence term, as

$$
\frac{\partial \rho}{\partial t}+\mathbf{u} \cdot \nabla \rho+\rho \nabla \cdot \mathbf{u}=0
$$

The first term in the above equation describes the local temporal variations of the fluid density, while the second term represents the rate of change of the fluid density at a fixed point in space $\mathbf{x}$ due to transport (or advection) of the fluid elements. It is convenient to 
define the so-called material derivative as

$$
\frac{d}{d t} \doteq \frac{\partial}{\partial t}+\mathbf{u} \cdot \nabla
$$

such that Equation (2.2) shortly reads

$$
\frac{1}{\rho} \frac{d \rho}{d t}+\nabla \cdot \mathbf{u}=0
$$

The equation of motion for an inviscid fluid, describing the conservation of momentum, is the Euler equation

$$
\frac{\partial \mathbf{u}}{\partial t}+(\mathbf{u} \cdot \nabla) \mathbf{u}=-\frac{1}{\rho} \nabla p,
$$

where $p$ denotes the hydrostatic fluid pressure. This equation describes the fluid flow without taking into account any process of energy dissipation (ideal fluid). Using tensor notation, Euler equation reads

$$
\frac{\partial \rho u_{i}}{\partial t}=-\frac{\partial \Pi_{i j}}{\partial x_{j}}
$$

where the second rank tensor $\Pi_{i j}$ is defined by

$$
\Pi_{i j} \doteq p \delta_{i j}+\rho u_{i} u_{j} .
$$

The physical meaning of the different terms in (2.6) becomes clear after a volume integration. Integrating both sides of this equation over the fluid volume $\mathcal{V}$ and using the divergence theorem, we are lead to

$$
\frac{\partial}{\partial t} \int_{\mathcal{V}} \rho u_{i} d V=\oint_{\partial \mathcal{V}} \Pi_{i j} d S_{j}
$$

where $\partial \mathcal{V}$ denotes the closed boundary surface of the volume $\mathcal{V}$ and $d S_{j}=d S n_{j}$ with $n_{j}$ the outward normal to the surface element. Since the left-hand side of (2.8) is the local rate of change of the $i$-th component of the momentum in the fluid volume, $\Pi_{i j}$ is the $i$-th component of the amount of momentum flowing per unit time through the unit area perpendicular to the $n_{j}$ direction. For this reason $\Pi_{i j}$ is called the momentum flux density tensor or stress tensor. Each diagonal component of $\Pi_{i j}$ gives the normal component of the internal surface forces acting on a surface parallel to one of the co-ordinate planes (normal stresses). The non-diagonal components of $\Pi_{i j}$ describe the surface forces acting perpendicularly to the direction of fluid motions. The latter surface forces are setup by shearing motions of fluid layers and are therefore called tangential stresses. It is evident that the stress tensor (2.7) is symmetric, that is $\Pi_{i j}=\Pi_{j i}$. This means that the transfer of momentum in ideal fluids is completely reversible as expected.

In real (and planetary) flows internal friction and heat exchange between different parts of the fluid lead to energy dissipation and therefore Equations (2.6) and (2.7) require some modifications. Equation (2.7) can be corrected introducing a new term $-\sigma_{i j}^{\prime}$ which describes the amount of momentum per unit time and unit area lost in the fluid motions due to internal friction (viscosity), namely

$$
\Pi_{i j}=p \delta_{i j}-\sigma_{i j}^{\prime}+\rho u_{i} u_{j} .
$$


The derivation of the explicit form of $\sigma_{i j}^{\prime}$ is phenomenological and can be found in classical textbooks of fluid dynamics (see, e.g., Batchelor 2000, Chapter 3). The resulting expression reads

$$
\sigma_{i j}^{\prime}=\mu\left(\frac{\partial u_{i}}{\partial x_{j}}+\frac{\partial u_{j}}{\partial x_{i}}\right)+\left(\zeta-\frac{2}{3} \mu\right) \frac{\partial u_{k}}{\partial x_{k}} \delta_{i j}
$$

where the positive constants $\mu$ and $\zeta$ are the so-called coefficients of viscosity and second viscosity respectively. Note that the above expression depends only on the spatial derivatives of the fluid velocity. This is due to the fact that internal friction occurs only when relative motions of different parts of the fluid are present. Such velocity gradients have been assumed to be small and therefore only first order derivatives are retained. The two terms collected in the first parenthesis of (2.10) describe the transport of momentum due to the shearing motions of different fluid layers. The local rate of expansion $\partial_{k} u_{k}$ is associated with the rate of change of the volume of fluid elements and also causes a transport of momentum which is taken into account in the last term of (2.10).

The other essential assumption used to derive (2.10) is the fluid isotropy. The molecular structure of the fluid can be reasonably considered statistically isotropic. Macroscopically, this implies that the stress generated in any element of the fluid is independent of the element orientation. Fluids for which the linear relation (2.10) holds are said to be Newtonian.

Substituting (2.9) into Euler equation (2.6) leads to the equation of motion of a viscous fluid

$$
\rho\left[\frac{\partial \mathbf{u}}{\partial t}+(\mathbf{u} \cdot \nabla) \mathbf{u}\right]=-\nabla p+\mu \nabla^{2} \mathbf{u}+\left(\zeta+\frac{\mu}{3}\right) \nabla(\nabla \cdot \mathbf{u})
$$

which is known as the Navier-Stokes equation.

In the presence of gravity, the additional force $\rho \mathbf{g}$ acts on any unit volume and the Navier-Stokes equation (2.75) modifies to

$$
\frac{\partial \mathbf{u}}{\partial t}+(\mathbf{u} \cdot \nabla) \mathbf{u}=-\frac{1}{\rho} \nabla p+\mathbf{g}+v \nabla^{2} \mathbf{u}+\left(\frac{\zeta}{\rho}+\frac{\nu}{3}\right) \nabla(\nabla \cdot \mathbf{u}),
$$

where the kinematic viscosity $v$ is defined as

$$
v \doteq \frac{\mu}{\rho} .
$$

The coefficient $\mu$ itself is called the dynamic viscosity.

\subsection{Thermodynamic equations}

As already anticipated in Section 1.2, the main drivers of fluid motions in planetary cores are thermal and compositional convection. In thermal convection buoyancy forces are setup by temperature gradients, while in compositional convection they are caused by the release of light elements at the inner-core boundary. In this section, the compositional driving is neglected and we concentrate on thermal convection. Starting from the principles of thermodynamics, an evolution equation for the temperature is derived in the following. 
Consider a thermodynamic transformation between two neighboring equilibrium states. During such transformation, the system energy varies according to the first law of thermodynamics

$$
d U=d Q+d W
$$

where $d U$ represents the variation in internal energy per unit mass of the fluid, $d Q$ the heat exchanged by the system per unit mass and $d W$ the work per unit mass done by the fluid during the transformation.

Consider now a mechanical compression of the fluid. If the transformation is reversible, the work done on the fluid is $-p d V$. Since the volume is taken per unit mass, $V=\rho^{-1}$ and (2.14) becomes

$$
d U=d Q+\frac{p}{\rho^{2}} d \rho .
$$

In order to derive an evolution equation for the internal energy $U$ of the system from the above expression, variations in the heat $Q$ need to be characterized in terms of changes in two of the fundamental variables $(T, p, \rho)$. The entropy $S$ of the system, defined in a reversible transformation from an equilibrium state to another, is introduced as an extensive quantity by $T d S=d Q$. Regarding $S$ as a function of temperature and pressure, the entropy variations can be written as

$$
d S=\left(\frac{\partial S}{\partial T}\right)_{p} d T+\left(\frac{\partial S}{\partial p}\right)_{T} d p,
$$

where the subscripts indicate that the terms in parenthesis have to be taken at constant pressure and temperature respectively. Introducing the heat capacity at constant pressure $c_{p}$ as the amount of heat exchanged by the system due to a change in temperature at constant pressure, we have

$$
c_{p} \doteq\left(\frac{\partial Q}{\partial T}\right)_{p}=T\left(\frac{\partial S}{\partial T}\right)_{p} .
$$

The first term in parenthesis on the right hand side (RHS) of (2.16) is thus proportional to $c_{p}$.

Using one of Maxwell's relations of thermodynamics, the second term on the RHS of (2.16) can be simplified as

$$
\left(\frac{\partial S}{\partial p}\right)_{T}=-\left(\frac{\partial V}{\partial T}\right)_{p}=\frac{1}{\rho^{2}}\left(\frac{\partial \rho}{\partial T}\right)_{p}
$$

Defining the coefficient of thermal expansion of the fluid $\alpha$ as

$$
\alpha \doteq \frac{1}{V}\left(\frac{\partial V}{\partial T}\right)_{p}=-\frac{1}{\rho}\left(\frac{\partial \rho}{\partial T}\right)_{p},
$$

expression (2.18) reads

$$
\left(\frac{\partial S}{\partial p}\right)_{T}=-\frac{\alpha}{\rho}
$$

Substituting (2.17) and (2.20) into Equation (2.16) finally leads to the expression for the entropy variations

$$
d S=\frac{c_{p}}{T} d T-\frac{\alpha}{\rho} d p .
$$


The evolution equation of entropy is obtained dividing both sides of the above expression by $d t$

$$
T \frac{d S}{d t}=c_{p} \frac{d T}{d t}-\frac{\alpha}{\rho} T \frac{d p}{d t} .
$$

In planetary cores, heat variations $d Q$ are influenced by internal sources of thermal energy. Introducing the local heat flux $\mathbf{q}$ and a term $h$ which includes all the internal sources and sinks of heat per unit volume, the rate of change of heat can be generally written as

$$
\rho \frac{d Q}{d t}=-\nabla \cdot \mathbf{q}+h .
$$

Fourier's law of heat conduction provides $\mathbf{q}=-k \nabla T$, where $k$ is the fluid thermal conductivity, and Equation (2.23) becomes

$$
\rho \frac{d Q}{d t}=\nabla \cdot(k \nabla T)+h .
$$

Assuming a fluid with constant thermal conductivity $k$ and using the definition of entropy, Equation (2.24) can be rewritten as

$$
\rho T \frac{d S}{d t}=k \nabla^{2} T+h .
$$

Comparing the above equation with (2.22), we are finally lead to the evolution equation for the temperature $T$

$$
\rho c_{p} \frac{d T}{d t}-\alpha T \frac{d p}{d t}=k \nabla^{2} T+h .
$$

If the time derivatives in the above equation are interpreted as material derivatives, (2.26) represents the general equation describing the effect of heat conduction in a moving fluid with a constant thermal conductivity $k$.

In planetary iron cores, different physical mechanisms contribute as sources or sinks of heat in the term $h$. Among the heat losses, the core secular cooling is perhaps the most important one. As the core cools down, however, it gradually crystallize at the top of the inner-core boundary thus releasing latent heat in the liquid outer core. This represents an important source of thermal energy driving convection in the Earth's outer core. On the other hand, the freezing inner core cannot dissolve very efficiently light elements thus causing their expulsion in the liquid outer core. Such chemical differentiation between the lighter fluid layers close to the inner core boundary and the overlying liquid can help the fluid to become buoyant (compositional convection). Another important contribution to the internal heat production comes from radioactive decay. This can be considered as a uniform heat source since vigorous convection is expected to homogeneously distribute radiogenic elements in the outer core. For a viscous and magnetically conducting fluid, heat due to viscous and Ohmic dissipation may also contribute. A detailed discussion on the relative importance of the different sources and sinks of heat in the Earth's core can be found in Nimmo (2007).

To summarize, the equations governing fluid motions in planetary cores are: the continuity equation (2.4), the Navier-Stokes equation (2.12) and the temperature equation (2.26). They must be complemented by an equation of state (EOS)

$$
\rho=\rho(T, p)
$$


which closes the system. In principle, the above equations could be used to solve numerically for thermal convection in the core. They describe physical processes in a wide range of characteristic time scales varying, in the case of Earth's core, from few minutes (acoustic/seismic) to hundreds of years (convection turnover time). Moreover, the temperature perturbations $T^{\prime}$ driving convection are a very small fraction of the mean core temperature. Simple orders of magnitude estimates reveal indeed $T^{\prime} \sim 7 \times 10^{-5} \mathrm{~K}$ (Jones 2007). From a practical point of view, numerical errors of the same magnitude for the temperature would lead to unreliable solutions. In order to model convection, it is therefore advantageous to study only the small deviations from an equilibrium state (reference state) induced by convective fluctuations. Simplified forms of the above equations, obtained upon linearization of the thermodynamic relations, are discussed in the next section. More precisely, we derive the equations governing thermal convection under the Boussinesq approximation and we discuss the validity of such approximation in the case of Earth's core.

\subsection{Equations for the convective perturbations}

In order to derive the equations for the convective perturbations, we have first to specify a reference state about which linearizing the thermodynamic relations. The contributions to the stationary reference state (indicated by the subscript ${ }_{0}$ ) are distinguished from the timedependent convective perturbations (indicated by the superscript '). It is useful to separate further the reference state into the sum of a (constant) mean background contribution and its spatial variations. The density $\rho$, the pressure $p$ and the temperature $T$ of the fluid can then be written as

$$
\begin{aligned}
\rho(\mathbf{r}, t) & =\rho_{m}+\rho_{a}(\mathbf{r})+\rho^{\prime}(\mathbf{r}, t) \\
p(\mathbf{r}, t) & =p_{m}+p_{a}(\mathbf{r})+p^{\prime}(\mathbf{r}, t) \\
T(\mathbf{r}, t) & =T_{m}+T_{a}(\mathbf{r})+T^{\prime}(\mathbf{r}, t)
\end{aligned}
$$

where the subscript ${ }_{m}$ refers to a (constant) spatial average over the fluid domain of the corresponding quantity, ${ }_{a}$ to the variations in the absence of motion and the primed quantities to the fluctuations resulting from convective motions. Since by assumption the reference state is steady $\left(\mathbf{u}_{0}=\mathbf{0}\right)$, we denote the fluid velocity in the perturbed state by $\mathbf{u}$ for simplicity of notation.

For the convective motions to be slow, it is necessary that the reference state is in quasi-equilibrium. In the absence of motions, Euler equation (2.5) in the presence of gravity reduces to the hydrostatic balance

$$
-\nabla p_{a}+\rho_{0} \mathbf{g}=0
$$

where $\rho_{0}=\rho_{m}+\rho_{a}$. The above equation describes the mechanical equilibrium of the fluid in the reference state.

There are different ways of imposing the thermal quasi-equilibrium of the reference state, but the most natural choice for convection problems is to consider a state in which heat is transported only by conduction. In the Earth's outer core, heat is transported far more effectively by convection than by conduction. Considering a turbulent thermal diffusivity $\kappa_{T} \sim 2 \mathrm{~m}^{2} / \mathrm{s}$ (Jones 2007), the characteristic time scale of thermal diffusion 
processes in the core is $d^{2} / \kappa_{T}$, where $d$ is the outer shell thickness, and amounts to about $80 \mathrm{kyr}$. Since the convective turnover time can be estimated to be of a few hundreds years, this separation of time scales allows to consider the conduction state as a steady background for convection. The thermodynamic transformations of the fluid core due to thermal diffusion processes can therefore be considered adiabatic with respect to the convective time scales. This implies that the transformations of the reference state are isentropic ( $d S=0)$ and, consequently, the evolution equation for the temperature (2.26) consequently reduces to

$$
k \nabla T_{a}=-h_{0} .
$$

The above equation describes the thermodynamic balance of the adiabatic reference state between thermal diffusion and the heat coming from sources distributed within the core and, eventually, from the boundaries.

Following Spiegel and Veronis (1960), we introduce the scale heights

$$
H_{f} \doteq\left|\frac{1}{f_{m}} \frac{d f_{a}}{d r}\right|^{-1}
$$

where $f$ denotes any of the thermodynamic variables $\rho, p, T$ and $r$ the radial distance. The basic assumption of the Boussinesq approximation is that the reference thermodynamic quantities vary on length scales much larger than the largest linear dimension of the system. In other words, the fluid is assumed to be confined in a spherical shell whose thickness $d$ is such that

$$
d \ll H_{f} .
$$

This is often referred to as the thin-shell approximation.

The validity of such approximation in the case of Earth's core can be examined estimating, for the different thermodynamic quantities, the scale heights (2.31) as

$$
H_{f} \sim\left(\frac{1}{f_{m}} \frac{\left|\Delta f_{a}\right|}{d}\right)^{-1} .
$$

Assuming a spherically symmetric outer core of thickness $d, \Delta f_{a}$ in the above expression is the change of $f$ across the fluid shell $f\left(r_{o}\right)-f\left(r_{i}\right)$ and $f_{m}$ is its mean value $\left[f\left(r_{o}\right)+\right.$ $\left.f\left(r_{i}\right)\right] / 2$. Using typical values for the density $\rho$, the temperature $T$ and the pressure $p$ at the inner core boundary $r_{i}$ and at the core-mantle boundary $r_{o}$ (Olson 2007, Tables 2 and $3)$, the estimated scale heights of the respective quantities are

$$
\begin{aligned}
H_{\rho} / d & \sim 15.6 \\
H_{T} / d & \sim 3.0 \\
H_{p} / d & \sim 1.2 .
\end{aligned}
$$

Since all the scale heights exceed the thickness of the Earth's outer core, the thin-shell approximation (2.32) can be considered to be at least marginally satisfied.

We now define for convenience

$$
\epsilon_{a} \doteq \frac{\left|\Delta \rho_{a}\right|}{\rho_{m}}
$$


which, using (2.33) and according to the above estimates, results in $\epsilon_{a} \sim d / H_{\rho} \approx 6 \cdot 10^{-2}$. Let the reference state described by equations (2.29) and (2.30) be slightly perturbed. Assuming that the density fluctuations $\rho^{\prime}$ do not exceed in amplitude the static background density $\rho_{m}$, we can write

$$
\left|\frac{\rho^{\prime}}{\rho_{m}}\right| \doteq \epsilon^{\prime} \lesssim O\left(\epsilon_{a}\right) .
$$

Substituting the decompositions (2.28) into the continuity equation (2.2) yields

$$
\frac{\partial \rho^{\prime}}{\partial t}+\mathbf{u} \cdot \nabla\left(\rho_{a}+\rho^{\prime}\right)+\rho \nabla \cdot \mathbf{u}=0 .
$$

Dividing both sides of the above equation by $\rho_{m}$ and using (2.34) leads to

$$
\frac{\partial}{\partial t}\left(\frac{\rho^{\prime}}{\rho_{m}}\right)+(\mathbf{u} \cdot \nabla+\nabla \cdot \mathbf{u})\left(\frac{\rho_{a}}{\Delta \rho_{a}} \epsilon_{a}+\frac{\rho^{\prime}}{\rho_{m}}\right)+\nabla \cdot \mathbf{u}=0 .
$$

To order $\epsilon_{a}$, the continuity equation (2.37) reduces therefore to the condition for an incompressible flow

$$
\nabla \cdot \mathbf{u}=0 .
$$

We now derive the equation of motion for the convective perturbations. Since by assumption the density variations of the reference state and the density perturbations are small compared to the mean background density $\rho_{m}$, the EOS (2.27) can be linearized. A Taylor expansion of $\rho=\rho(T, p)$ around $\left(\rho_{m}, T_{m}, p_{m}\right)$ up to the first order yields

$$
\begin{aligned}
\rho & \simeq \rho_{m}\left[1+\frac{1}{\rho_{m}}\left(\frac{\partial \rho}{\partial T}\right)_{p_{m}}\left(T-T_{m}\right)+\frac{1}{\rho_{m}}\left(\frac{\partial \rho}{\partial p}\right)_{T_{m}}\left(p-p_{m}\right)\right] \\
& =\rho_{m}\left[1-\alpha\left(T-T_{m}\right)+\beta\left(p-p_{m}\right)\right],
\end{aligned}
$$

where $\alpha$ is the thermal expansion coefficient defined in (2.19) and $\beta$ the isothermal compression coefficient

$$
\beta \doteq \frac{1}{\rho}\left(\frac{\partial \rho}{\partial p}\right)_{T}
$$

Substituting (2.28) in the above expansion leads to

$$
\frac{\rho_{a}}{\rho_{m}}+\frac{\rho^{\prime}}{\rho_{m}}=-\alpha\left(T_{a}+T^{\prime}\right)+\beta\left(p_{a}+p^{\prime}\right) .
$$

Since the adiabatic reference state is stationary, the first term on the left hand side (LHS) of the above equation must equal the time independent terms $-\alpha T_{a}+\beta p_{a}$ on the RHS. As a consequence, (2.40) can be separated into the two equations

$$
\begin{aligned}
& \frac{\rho_{a}}{\rho_{m}}=-\alpha T_{a}+\beta p_{a} \\
& \frac{\rho^{\prime}}{\rho_{m}}=-\alpha T^{\prime}+\beta p^{\prime} .
\end{aligned}
$$

These are the linearized thermodynamic relations which will be used in the following. 
Since the flow is divergence-free, the Navier-Stokes equation 2.12) reduces to

$$
\rho \frac{d \mathbf{u}}{d t}=-\nabla p+\rho \mathbf{g}+\rho v \nabla^{2} \mathbf{u} .
$$

Expanding density and pressure as in (2.28) and using the hydrostatic balance of the reference state (2.29), the above equation reads

$$
\frac{\rho}{\rho_{m}} \frac{d \mathbf{u}}{d t}=-\frac{1}{\rho_{m}} \nabla p^{\prime}+\frac{\rho^{\prime}}{\rho_{m}} \mathbf{g}+\frac{\rho}{\rho_{m}} \nu \nabla^{2} \mathbf{u}
$$

where we divided both sides by $\rho_{m}$.

Expanding the density $\rho$ as in (2.28a) and using the definition (2.34) yields

$$
\left(1+\epsilon_{a} \frac{\rho_{a}}{\left|\Delta \rho_{a}\right|}+\frac{\rho^{\prime}}{\rho_{m}}\right)\left(\frac{d \mathbf{u}}{d t}-v \nabla^{2} \mathbf{u}\right)=-\frac{1}{\rho_{m}} \nabla p^{\prime}+\frac{\rho^{\prime}}{\rho_{m}} \mathbf{g} .
$$

The second and third terms in the first parenthesis on the LHS of the above equation are $O\left(\epsilon_{a}\right)$ and $O\left(\epsilon^{\prime}\right)$ respectively, while the buoyancy term on the RHS has a prefactor which is $O\left(\epsilon_{a}\right)$. If we neglect these higher order terms with the exception of the buoyancy term necessary to drive convection, Equation (2.44) reduces to

$$
\frac{d \mathbf{u}}{d t}=-\frac{1}{\rho_{m}} \nabla p^{\prime}+\frac{\rho^{\prime}}{\rho_{m}} \mathbf{g}+v \nabla^{2} \mathbf{u}
$$

where $\rho^{\prime}$ is related to the pressure and temperature perturbations by the linearized thermodynamic equation (2.41b).

The first reason to retain the buoyancy term in (2.44) is physical and consists in the fact that buoyancy forces must be present in the equation of motion when studying convection problems. On the other hand, since fluid motions are driven by density fluctuations, the characteristic acceleration of the fluid must be of order $\rho^{\prime} / \rho_{m} \mathbf{g}$ which implies

$$
\left|\frac{\partial \mathbf{u}}{\partial t}\right| /|\mathbf{g}| \sim O\left(\epsilon^{\prime}\right) \ll 1 .
$$

The acceleration of gravity is therefore much larger than the characteristic fluid acceleration due to convective motions. The buoyancy term of Equation 2.45) remains therefore finite even though its prefactor is $O\left(\epsilon_{a}\right)$. Only density variations coupled to the gravitational acceleration in the buoyancy force can then be retained, while all the other density variations can be neglected. This represents the core assumption of the Boussinesq approximation.

Equation (2.45) can be simplified further analyzing the separation of scales on which density, temperature and pressure vary. The estimated scale heights of these thermodynamic quantities in the case of Earth's core satisfy, as shown above, $H_{\rho}<H_{T}<H_{p}$. Therefore, the background density varies more effectively due to temperature changes rather than due to pressure fluctuations. Density changes due to pressure fluctuations, quantified by the compression coefficient $\beta$, are then considered negligible. In this approximation, the thermodynamic equation equation (2.41b) reduces to

$$
\frac{\rho^{\prime}}{\rho_{m}}=-\alpha T^{\prime}
$$


The fact that the background density variations due to convection are caused only by temperature changes is the last fundamental assumption of the Boussinesq approximation. Note that this argument does not apply to Equation (2.41a), since $p_{a}$ provides the major contribution in the hydrostatic balance of the reference state described by (2.29). Substituting (2.46) into (2.45), we finally obtain the equation of motion for the convective perturbations in the Boussinesq approximation which reads

$$
\frac{\partial \mathbf{u}}{\partial t}+\mathbf{u} \cdot \nabla \mathbf{u}=-\frac{1}{\rho_{m}} \nabla p^{\prime}-\alpha T^{\prime} \mathbf{g}+\nu \nabla^{2} \mathbf{u} .
$$

We now derive the evolution equation for the convective temperature fluctuations $T^{\prime}$ starting from the temperature evolution equation (2.26) which holds for a fluid with constant thermal conductivity $k$. Expanding the temperature $T$ as in (2.28c) and the heat sources as $h=h_{0}(\mathbf{r})+h^{\prime}(\mathbf{r}, t)$, Equation (2.26) reads

$$
\rho c_{p} \frac{d T}{d t}-\alpha T \frac{d p}{d t}=k \nabla^{2} T_{a}+h_{0}+k \nabla^{2} T^{\prime}+h^{\prime} .
$$

Since the reference state is a conduction state, i.e. Equation (2.30) holds, the above equation simplifies to

$$
\rho c_{p} \frac{d T}{d t}-\alpha T \frac{d p}{d t}=k \nabla^{2} T^{\prime}+h^{\prime}
$$

Consider now the second term on the LHS of (2.49). Since the pressure $p$ is a function of density and temperature, the material derivative $d p / d t$ can be written as

$$
\begin{aligned}
\frac{d p}{d t} & =\left(\frac{\partial p}{\partial \rho}\right)_{T} \frac{d \rho}{d t}+\left(\frac{\partial p}{\partial T}\right)_{\rho} \frac{d T}{d t} \\
& =-\left(\frac{\partial p}{\partial \rho}\right)_{T} \rho \nabla \cdot \mathbf{u}+\left(\frac{\partial p}{\partial T}\right)_{\rho} \frac{d T}{d t}
\end{aligned}
$$

by means of the continuity equation (2.4). To order $\epsilon_{a}$, the velocity field for the convective perturbations is divergence-free (Equation 2.38), and the first term on the RHS of (2.51) can be neglected.

Pressure variations due to temperature changes constitute the prefactor of the second term on the RHS of (2.51) and can be estimated using the linearized thermodynamic relations (2.41a) and (2.46). These variations are negligible for the convective perturbations by assumption (Equation 2.46) and must be proportional to $\alpha$ for the adiabatic reference state (Equation 2.41a). As an order of magnitude estimate, the pressure term on the LHS of Equation (2.49) can then be written as

$$
\left|\alpha T \frac{d p}{d t}\right| \sim \alpha^{2} T \sim O\left(\epsilon_{a}^{2}\right)
$$

where we regarded $\alpha \sim O\left(\epsilon_{a}\right)$. This term can be therefore neglected and Equation 2.49) simplifies to

$$
\rho c_{p} \frac{d T}{d t}=k \nabla^{2} T^{\prime}+h^{\prime} .
$$

Expanding the density $\rho$ as in (2.28a and dividing both sides of the above equation by $\rho_{m}$ yields to

$$
\left(1+\epsilon \frac{\rho_{a}}{\Delta \rho_{a}}+\frac{\rho^{\prime}}{\rho_{m}}\right) c_{p} \frac{d T}{d t}=\frac{k}{\rho_{m}} \nabla^{2} T^{\prime}+\frac{h^{\prime}}{\rho_{m}} .
$$


Neglecting terms of order $\epsilon_{a}$ and higher, the evolution equation for the temperature perturbations finally reads

$$
\rho_{m} c_{p} \frac{d T^{\prime}}{d t}=\kappa \nabla^{2} T^{\prime}+h^{\prime}
$$

The hydrodynamical equations for the convective perturbations derived in the Boussinesq approximation are then

$$
\begin{aligned}
\frac{d \mathbf{u}}{d t} & =-\frac{1}{\rho} \nabla \Pi-\alpha T \mathbf{g}+\nu \nabla^{2} \mathbf{u} \\
\nabla \cdot \mathbf{u} & =0 \\
\rho c_{p} \frac{d T}{d t} & =\kappa \nabla^{2} T+h
\end{aligned}
$$

where the primes are suppressed in the notation for simplicity. The pressure fluctuations $p^{\prime}$ are renamed as $\Pi$ in order to distinguish them from the hydrostatic contributions, and $\rho$ denotes the (constant) mean fluid density.

\subsection{Rotating frame of reference}

The equations (2.55) derived in the previous sections are valid in an inertial frame of reference. On Earth, we naturally observe fluid motions on a reference frame fixed at the surface and therefore rotating with respect to an inertial frame. Rather than dealing with moving boundaries, it is more convenient to write the Navier-Stokes equation (2.55a) in such non-inertial frame. The additional fictitious forces entering this equation play a fundamental role in shaping the flow.

Consider a frame of reference $S_{R}$ rotating about the $z$-axis at a uniform angular velocity $\boldsymbol{\Omega}=\Omega \hat{\mathbf{e}}_{z}$ with respect to a (fixed) inertial frame $S_{I}$. Let $S_{R}$ and $S_{I}$ have a common origin of coordinates $O$ and denote the position vector of a point in space with respect to the origin $O$ with $\mathbf{r}$. The rate of change of $\mathbf{r}$ as seen by an inertial observer is related to the rate of change in the rotating frame $S_{R}$ by (see, e.g., Batchelor 2000, Chapter 4)

$$
\left(\frac{d \mathbf{r}}{d t}\right)_{I}=\left(\frac{d \mathbf{r}}{d t}\right)_{R}+\boldsymbol{\Omega} \times \mathbf{r} .
$$

The first term on the RHS describes the variation of position with respect to $S_{R}$, while the second term arises from the rotation of the unit vectors of $S_{R}$ as seen by an observer fixed with respect to $S_{I}$. The above equation obviously represents the transformation law for the velocity between the two reference frames:

$$
\mathbf{u}_{I}=\mathbf{u}_{R}+\boldsymbol{\Omega} \times \mathbf{r} .
$$

The acceleration measured by an inertial observer $\mathbf{a}_{I}$ can be obtained applying the transformation (2.56) on $\mathbf{u}_{I}$, thus

$$
\left(\frac{d \mathbf{u}_{I}}{d t}\right)_{I}=\left(\frac{d \mathbf{u}_{I}}{d t}\right)_{R}+\boldsymbol{\Omega} \times \mathbf{r} .
$$


Using (2.57) in the above equation yields

$$
\frac{d \mathbf{u}_{I}}{d t}=\left(\frac{d \mathbf{u}_{R}}{d t}\right)_{R}+\mathbf{\Omega} \times\left(\frac{d \mathbf{r}}{d t}\right)_{R}+\mathbf{\Omega} \times \mathbf{u}_{R}+\mathbf{\Omega} \times(\boldsymbol{\Omega} \times \mathbf{r}),
$$

where the rotation rate has been assumed to be constant in time. This represents the transformation law for the acceleration between the two reference frames which more compactly reads

$$
\mathbf{a}_{I}=\mathbf{a}_{R}+2 \boldsymbol{\Omega} \times \mathbf{u}_{R}+\boldsymbol{\Omega} \times(\boldsymbol{\Omega} \times \mathbf{r}) .
$$

The difference between absolute and relative accelerations consists of two terms. The first, proportional to the rotation rate and to the velocity, is called the Coriolis acceleration; the second is the centripetal acceleration $\mathbf{a}_{c}$ and arises even in the absence of motions. This last term can be written as the gradient of a scalar potential function $\phi_{c}$ (centrifugal potential) which satisfies

$$
\mathbf{\Omega} \times(\mathbf{\Omega} \times \mathbf{r})=-\nabla\left[\frac{(\boldsymbol{\Omega} \times \mathbf{r})^{2}}{2}\right]
$$

Using the transformation law (2.59) into the Navier-Stokes equation (2.55a and expressing the centripetal acceleration as in (2.60), we are finally lead to the equation of motion for a convecting fluid in the Boussinesq approximation in a rotating frame of reference

$$
\frac{d \mathbf{u}}{d t}=-\frac{1}{\rho} \nabla \Pi^{*}-2 \mathbf{\Omega} \times \mathbf{u}-\alpha T \mathbf{g}+v \nabla^{2} \mathbf{u},
$$

where $\Pi^{*}$ is the modified pressure $\Pi^{*} \doteq \Pi-\rho(\boldsymbol{\Omega} \times \mathbf{r})^{2} / 2$ which includes centrifugal forces.

\subsection{The magnetic induction equation}

In this section the evolution equation for the magnetic field, known as the induction equation, is introduced. We concern here with conducting and non-magnetic materials. Electromagnetic phenomena in such media are described by Maxwell's equations which, in the Gaussian unit system, read

$$
\begin{aligned}
\nabla \cdot \mathbf{E} & =4 \pi \rho_{e} \\
\nabla \times \mathbf{E} & =-\frac{1}{c} \frac{\partial \mathbf{B}}{\partial t} \\
\nabla \cdot \mathbf{B} & =0 \\
\nabla \times \mathbf{B} & =\frac{4 \pi}{c} \mathbf{J}+\frac{1}{c} \frac{\partial \mathbf{E}}{\partial t} .
\end{aligned}
$$

where $\mathbf{E}$ is the electric field, $\mathbf{B}$ the magnetic field, $\rho_{e}$ the distribution of electric charges, $\mathbf{J}$ the current density and $c$ the speed of light.

In magnetohydrodynamics (MHD) a quasi-steady approximation of the above equations is used. In this approximation the fluid system is considered to be stationary on light travel times, thus neglecting the propagation of eventual electromagnetic waves. In planetary cores, fluid motions are typically of the order of few millimeters per second and 
therefore this approximation is valid to a large extent. If $\ell$ is a characteristic length scale of the system and $\tau$ the time scale of field variations, Faraday's law (2.62b) allow us to write

$$
\frac{|\mathbf{E}|}{|\mathbf{B}|} \sim \frac{v}{c} \ll 1
$$

where $v \doteq \ell / \tau$ defines a characteristic system velocity. The above estimate implies $|\mathbf{E}| \ll$ $|\mathbf{B}|$, thus the electric field plays only a minor role compared to the magnetic field. By means of 2.62a), we can conclude that the charge density $\rho_{e}$ is not significant in MHD. Physical effects due to charge separation in the conductive fluid act on time scales of the order of the electron gyro-frequency, thus much faster than typical fluid motions. Since $\rho_{e}$ is very small in the interior of the conductor, Gauss' law (2.62a can be simply dropped. In MHD electric fields are therefore generated only via induction effects due to time variable magnetic fields according to Equation (2.62b).

The displacement current $c^{-1} \partial_{t} \mathbf{E}$ in the Ampère-Maxwell equation (2.62d) can also be neglected. As an order of magnitude estimate, compare the displacement current with the first term on the LHS of Equation (2.62d):

$$
\frac{\left|c^{-1} \partial_{t} \mathbf{E}\right|}{|\nabla \times \mathbf{B}|} \sim \frac{v}{c} \frac{|\mathbf{E}|}{|\mathbf{B}|} \sim \frac{v^{2}}{c^{2}} \ll 1 .
$$

This demonstrates that Maxwell's correction in Equation (2.62d) is not required in MHD.

Therefore, the (pre-Maxwell) electrodynamic equations used in MHD are

$$
\begin{aligned}
\nabla \times \mathbf{E} & =-\frac{1}{c} \frac{\partial \mathbf{B}}{\partial t} \\
\nabla \cdot \mathbf{B} & =0 \\
\nabla \times \mathbf{B} & =\frac{4 \pi}{c} \mathbf{J} .
\end{aligned}
$$

It is interesting to note that taking the divergence of both sides of Equation (2.64c) yields to

$$
\nabla \cdot \mathbf{J}=0
$$

which expresses the solenoidal character of the density current and agrees, as expected, with the fact that no free charge density $\rho_{e}$ is present in MHD.

We now derive the magnetic induction equation. Consider a fluid conductor with an electrical conductivity $\sigma$. If the conductor is at rest, the following (phenomenological) relationship, known as Ohm's law, holds

$$
\mathbf{J}=\sigma \mathbf{E} .
$$

In a reference frame moving with the fluid conductor, it can be expected that such relationship is still locally valid. If $\mathbf{J}_{R}$ and $\mathbf{E}_{R}$ are, respectively, the current density and the electric field as measured by an observer moving with respect to an inertial reference frame with the local fluid velocity $\mathbf{u}=\mathbf{u}(\mathbf{x})$, then $\mathbf{J}_{R}=\sigma \mathbf{E}_{R}$. Using the non-relativistic transformations for the electric and magnetic fields (see, e.g., Davidson 2001, Chapter 2), Ohm's law (2.66) can be written, relative to the fixed frame of reference, as

$$
\mathbf{J}=\sigma\left(\mathbf{E}+\frac{1}{c} \mathbf{u} \times \mathbf{B}\right) .
$$


The above equation represents the (generalized) Ohm's law for a moving conductor. Substituting (2.67) into Faraday's law (2.64a) gives

$$
\frac{\partial \mathbf{B}}{\partial t}=\nabla \times\left(\mathbf{u} \times \mathbf{B}-c \frac{\mathbf{J}}{\sigma}\right) .
$$

Using Ampère's law (2.64c) to eliminate the current density in the above equation yields to

$$
\frac{\partial \mathbf{B}}{\partial t}=\nabla \times(\mathbf{u} \times \mathbf{B})-\nabla \times(\eta \nabla \times \mathbf{B})
$$

where $\eta$ is the magnetic diffusivity of the fluid defined as

$$
\eta \doteq c^{2} / 4 \pi \sigma .
$$

Assuming $\eta$ is constant, the second term on the RHS of Equation (2.69) is $\eta \nabla \times(\nabla \times \mathbf{B})=$ $\eta\left(\nabla(\nabla \cdot \mathbf{B})-\nabla^{2} \mathbf{B}\right)=-\eta \nabla^{2} \mathbf{B}$ which finally leads to

$$
\frac{\partial \mathbf{B}}{\partial t}=\nabla \times(\mathbf{u} \times \mathbf{B})+\eta \nabla^{2} \mathbf{B} .
$$

The above equation governs the magnetic field evolution in a moving conductor with constant diffusivity $\eta$ and is called the magnetic induction equation.

If fluid motions were to be absent $(\mathbf{u}=\mathbf{0})$, the induction equation (2.71) would reduce to a diffusion equation and therefore $\mathbf{B}$ would decay exponentially. The rate of decay of the field is, as a simple dimensional analysis would suggest, $\tau_{\eta} \doteq \ell^{2} / \eta$ where $\ell$ is a characteristic length scale of the system. Using recent estimates of the electrical conductivity of iron at Earth's core conditions (Pozzo et al. 2012) and taking the outer core gap $d$ as the typical length scale $\ell$, the geomagnetic field is expected to decay by ohmic diffusion on time scales $\tau_{\eta} \simeq 244 \mathrm{kyr}$. Since we have evidences for an active magnetic field which go back to periods as old as 500 million years ago $(\mathrm{Ogg} 2012)$, the term $\nabla \times(\mathbf{u} \times \mathbf{B})$ in the induction equation (2.71) must act as a source of magnetic energy which prevents the field to decay by ohmic diffusion. As already discussed in Section 1.2, the idea that motions in an electrically conducting fluid might generate induced currents able to amplify and selfsustain an original seed field was firstly proposed by Larmor (1919). This hypothesis is now used in the study of magnetic field generation in planetary cores and stellar interiors, and is at the basis of modern hydromagnetic dynamo theory.

For a conducting fluid, the equation governing fluid motions (2.61) must be modified by considering the electromagnetic forces acting on the fluid conductor itself. These forces can be expressed per unit volume as (Davidson 2001)

$$
\mathbf{f}=\rho_{e} \mathbf{E}+\frac{1}{c} \mathbf{J} \times \mathbf{B}
$$

The first term represents the forces experienced by the free charges due to the presence of an electric field $\mathbf{E}$ (electrostatic force); the second term describes the contribution due to the motion of the charged particles into the magnetic field $\mathbf{B}$ (Lorentz force). As already discussed before, the total charge density $\rho_{e}$ is small in fluids with high electrical conductivity and therefore the electrostatic force can be expected to be negligible compared to the Lorentz force. In this approximation, Equation (2.72) simplifies to

$$
\mathbf{f} \simeq \frac{1}{4 \pi}(\nabla \times \mathbf{B}) \times \mathbf{B}
$$


where we expressed the density current $\mathbf{J}$ using Ampère's law (2.64c). On conversion to SI units, the volumetric Lorentz force becomes $\mathbf{f}=\mu_{0}^{-1}(\nabla \times \mathbf{B}) \times \mathbf{B}$ where $\mu_{0}=4 \pi \times$ $10^{-7} \mathrm{Vs} / \mathrm{Am}$ is the vacuum permeability.

Including the Lorentz force into the Navier-Stokes equation (2.61), we are finally lead to the equation of motion of a thermally convecting and conducting fluid in the presence of magnetic fields which reads

$$
\rho \frac{d \mathbf{u}}{d t}=-\nabla \Pi^{*}-2 \rho \mathbf{\Omega} \times \mathbf{u}-\alpha T \rho \mathbf{g}+\mu \nabla^{2} \mathbf{u}+\frac{1}{\mu_{0}}(\nabla \times \mathbf{B}) \times \mathbf{B} .
$$

\subsection{The non-dimensionalization}

In the previous sections, we derived a set of equations in the eight unknowns represented by the flow velocity $\mathbf{u}$, the magnetic field $\mathbf{B}$, the modified pressure $\Pi^{*}$ and the temperature perturbations $T$ which describe the dynamics of a conducting and thermally convecting fluid under the Boussinesq approximation. These equations, repeated here for convenience, are: the conservation of momentum

$$
\rho\left(\frac{\partial \mathbf{u}}{\partial t}+\mathbf{u} \cdot \nabla \mathbf{u}\right)=-\nabla \Pi^{*}-2 \rho \mathbf{\Omega} \times \mathbf{u}-\alpha T \rho \mathbf{g}+\mu \nabla^{2} \mathbf{u}+\frac{1}{\mu_{0}}(\nabla \times \mathbf{B}) \times \mathbf{B},
$$

the simplified continuity equation for a Boussinesq fluid

$$
\nabla \cdot \mathbf{u}=0
$$

the evolution equation of the temperature perturbations $T$ with respect to the adiabatic reference state without internal heat sources (or sinks)

$$
\rho c_{p}\left(\frac{\partial T}{\partial t}+\mathbf{u} \cdot \nabla\right) T=k \nabla^{2} T
$$

and the induction equation

$$
\frac{\partial \mathbf{B}}{\partial t}=\nabla \times(\mathbf{u} \times \mathbf{B})+\eta \nabla^{2} \mathbf{B} .
$$

These equations are supplemented by the solenoidal magnetic field condition

$$
\nabla \cdot \mathbf{B}=0
$$

It is generally useful to write differential equations using non-dimensional variables. This procedure introduces dimensionless parameters which measure the relative importance of the terms they multiply in the equations. We derive here the non-dimensional form of the above equations following the scaling used by Wicht (2005).

Any vector quantity $\mathbf{x}$ will be denoted hereafter by the product of its non-dimensional part $\hat{\mathbf{x}}$ with its (constant) units or dimensions $\tilde{x}$, i.e. $\mathbf{x}=\tilde{x} \hat{\mathbf{x}}$. The same notation holds for scalar quantities. Lengths are scaled with the outer core width $d \doteq r_{o}-r_{i}$, where $r_{o}$ and $r_{i}$ are the outer core and inner core radii respectively. Time is scaled in units of the outer core magnetic diffusion time $\tau_{\eta}=d^{2} / \eta$ introduced in Section2.5. A typical fluid velocity 
can then be defined as $\tilde{u}=d / \tau_{\eta}$ which yields $\mathbf{u}=\tilde{u} \hat{\mathbf{u}}=d / \tau_{\eta} \hat{\mathbf{u}}$. In such non-dimensional units, the time derivative, gradient and laplacian operators transform, respectively, as

$$
\begin{aligned}
\frac{\partial}{\partial t} & =\frac{1}{\tau_{\eta}} \frac{\partial}{\partial \hat{t}}, \\
\nabla & =\frac{1}{d} \hat{\nabla}, \\
\nabla^{2} & =\frac{1}{d^{2}} \hat{\nabla}^{2} .
\end{aligned}
$$

Let $\mathbf{B}=\tilde{B} \hat{\mathbf{B}}$, where $\tilde{B}$ is a typical magnetic field strength which will be defined later. Applying the above transformations to the induction equation (2.78) yields to

$$
\frac{\tilde{B}}{\tau_{\eta}} \frac{\partial \hat{\mathbf{B}}}{\partial \hat{t}}=\frac{\tilde{B}}{\tau_{\eta}} \hat{\nabla} \times(\hat{\mathbf{u}} \times \hat{\mathbf{B}})+\eta \frac{\tilde{B}}{d^{2}} \hat{\nabla}^{2} \hat{\mathbf{B}}
$$

Multiplying both sides by $\tau_{\eta} / \tilde{B}$, the above equation simplifies to

$$
\frac{\partial \hat{\mathbf{B}}}{\partial t}=\hat{\nabla} \times(\hat{\mathbf{u}} \times \hat{\mathbf{B}})+\hat{\nabla}^{2} \hat{\mathbf{B}} .
$$

Note that the non-dimensional induction equation (2.80) is formally equivalent to (2.78) when using the magnetic diffusion time as temporal units.

We proceed in non-dimentionalizing the evolution equation of temperature (2.77). The temperature perturbations $T$ are scaled with the super-adiabatic temperature contrast across the outer core $\Delta T$, such that $T=\Delta T \hat{T}$. Equation (2.77) can therefore be written as

$$
\frac{\Delta T}{\tau_{\eta}} \frac{\partial \hat{T}}{\partial \hat{t}}+\frac{\Delta T}{\tau_{\eta}}(\hat{\mathbf{u}} \cdot \hat{\nabla}) \hat{T}=\Delta T \frac{k}{\rho c_{p} d^{2}} \hat{\nabla}^{2} \hat{T} .
$$

The ratio of the thermal conductivity $k$ to the volumetric heat capacity $\rho c_{p}$ defines the thermal diffusivity $\kappa$

$$
\kappa \doteq \frac{k}{\rho c_{p}} .
$$

This quantity measures the fluid capability to conduct heat relative to its efficiency in retaining thermal energy. Multiplying both sides of Equation (2.81) by $\tau_{\eta} / \Delta T$ and using the definition of thermal diffusivity (2.82), we are lead to

$$
\frac{\partial \hat{T}}{\partial \hat{t}}+\hat{\mathbf{u}} \cdot \hat{\nabla} \hat{T}=\frac{\kappa}{\eta} \hat{\nabla}^{2} \hat{T}
$$

The dimensionless prefactor of the diffusion term in the above equation can be expressed in terms of two non-dimensional numbers. The Prandtl number Pr is defined by

$$
\operatorname{Pr}=\frac{\nu}{\kappa}
$$

and describes the relative importance of viscous diffusion to thermal diffusion in the fluid. In terms of time scales, $\operatorname{Pr}$ can be interpreted as the ratio of the thermal diffusion timescale $\tau_{\kappa}=d^{2} / \kappa$ to the viscous diffusion time scale $\tau_{v}=d^{2} / v$. The second non-dimensional 
number is the magnetic Prandtl number Pm which is the ratio of viscous diffusion to magnetic diffusion, namely

$$
P m=\frac{v}{\eta}
$$

and equals the time scales ratio $\tau_{\eta} / \tau_{\nu}$. Using these definitions, the non-dimensional form of the temperature equation (2.83) reads

$$
\frac{\partial \hat{T}}{\partial \hat{t}}+\hat{\mathbf{u}} \cdot \hat{\nabla} \hat{T}=\frac{P m}{P r} \hat{\nabla}^{2} \hat{T} .
$$

In planetary cores, the main driver of fluid motions (necessary for dynamo action) is convection. In order to maintain an operating dynamo, the thermal energy must diffuse on time scales much longer than the magnetic diffusion time. Therefore, we expect the ratio $\operatorname{Pm} / \operatorname{Pr}=\tau_{\eta} / \tau_{\kappa}$ to be small in planetary cores. In the case of Earth's core, for example, $P m$ is estimated to be $10^{-6}-10^{-5}$, while $\operatorname{Pr}$ is $0.1-1$ (Christensen 2011). Such low magnetic Prandtl numbers are practically unachievable in numerical dynamo simulations which generally use values from 0.1 to 10 . This means that the viscous diffusion time $\tau_{v}$ is underestimated by $5-6$ orders of magnitude in dynamo models. This leads to an enhanced fluid viscosity which damps the small and unresolvable scales present in real planetary cores.

We finally derive the non-dimensional form of the Navier-Stokes equation (2.75). The (constant) fluid density $\rho$ in (2.75) is assumed to be the mean outer core density $\rho_{o}$. The system is assumed to rotate uniformly about the $z$-axis with an angular velocity $\boldsymbol{\Omega}=\Omega \hat{\mathbf{e}}_{z}$, where $\Omega$ is the planetary rotation rate and $\hat{\mathbf{e}}_{z}$ the unit vector in the vertical direction. The magnetic field is scaled by $\tilde{B}=\left(\rho_{o} \Omega / \sigma\right)^{1 / 2}$, thus $\mathbf{B}=\left(\rho_{o} \Omega / \sigma\right)^{1 / 2} \hat{\mathbf{B}}$. The gravitational acceleration $\mathbf{g}$ is assumed to vary linearly with the radial distance, thus $\mathbf{g}=-g_{o} \mathbf{r} / r_{o}$ where $g_{o}$ is the value of the gravitational acceleration at the outer boundary. Using these scalings, Equation (2.75) reads

$$
\begin{aligned}
\rho_{o} \frac{d}{\tau_{\eta}^{2}}\left(\frac{\partial}{\partial \hat{t}}+\hat{\mathbf{u}} \cdot \hat{\nabla}\right) \hat{\mathbf{u}}= & \rho_{o} \Omega \frac{d}{\tau_{\eta}}\left(-\hat{\nabla} \hat{\Pi}^{*}-2 \mathbf{e}_{z} \times \hat{\mathbf{u}}\right)+ \\
& \alpha \Delta T \hat{T} \rho_{o} g_{o} \frac{\mathbf{r}}{r_{o}}+\mu \frac{1}{d \tau_{\eta}} \hat{\nabla}^{2} \hat{\mathbf{u}}+\frac{1}{\mu_{0}} \frac{1}{d} \frac{\rho_{o} \Omega}{\sigma}(\hat{\nabla} \times \hat{\mathbf{B}}) \times \hat{\mathbf{B}},
\end{aligned}
$$

where the same dimensional units for the Coriolis force and the modified (non-hydrostatic) pressure force have been adopted. Multiplying both sides of the above equation by $\tau_{\eta} / \rho_{o} \Omega d$ gives

$$
\frac{\eta}{d^{2} \Omega}\left(\frac{\partial}{\partial \hat{t}}+\hat{\mathbf{u}} \cdot \hat{\nabla}\right) \hat{\mathbf{u}}=-\hat{\nabla} \hat{\Pi}^{*}-2 \mathbf{e}_{z} \times \hat{\mathbf{u}}+\frac{\alpha g_{o} \Delta T d}{\Omega \eta} \frac{\mathbf{r}}{r_{o}} \hat{T}+\frac{v}{d^{2} \Omega} \hat{\nabla}^{2} \hat{\mathbf{u}}+(\hat{\nabla} \times \hat{\mathbf{B}}) \times \hat{\mathbf{B}}
$$

where we used the definitions of magnetic diffusivity $\eta=1 / \mu_{0} \sigma$ and of kinematic viscosity (2.13). The prefactor of the viscous diffusion term on the right of (2.85) is the Ekman number

$$
E=\frac{v}{\Omega d^{2}} .
$$

The Ekman number is therefore the ratio of the planetary rotation time $\Omega^{-1}$ to the viscous diffusion time scale $\tau_{v}$. It can also be interpreted as the ratio of viscous drag to the Coriolis 
force in the Navier-Stokes equation (2.75). In the case of Earth, $E$ is expected to be as low as $10^{-15}-10^{-14}$ (Christensen 2011). The dynamics of the fluid core is therefore strongly influenced by Coriolis forces, while viscous diffusion plays only a marginal role. Due to computational resource limits, self-consistent 3D dynamo simulations hardly reach an Ekman number of $10^{-6}-10^{-7}$ (Kageyama et al. 2008). This introduces an excess viscosity in the system which acts in damping the smallest and unresolvable scales occurring at realistic parameter values for the Earth's core.

The second dimensionless quantity on the RHS of Equation (2.85) multiplies the buoyancy term. In the study of convection problems, it is useful to introduce the (modified) Rayleigh number

$$
R a=\frac{\alpha g_{o} \Delta T d}{\Omega v}
$$

which represents a measure for the relative importance of buoyancy forces to viscous diffusion. $R a$ is therefore also a direct measure for the vigor of convection in the system. In order for convection to set in, the buoyancy force has to overcome stabilizing forces such as viscous diffusion, which tends to suppress fluid motions, and the Coriolis force, which tends to maintain the flow aligned with the rotation axis. The Rayleigh number in the Earth's core is estimated to be much larger than the critical value for the onset of convection, while dynamo models are less supercritical by nearly two orders of magnitude (Christensen 2011). Fluid motions in the Earth's outer core are therefore vigorously driven by convection, thus resulting in a high turbulent flow. Having introduced the Ekman number $E$ and the modified Rayleigh number $R a$, Equation (2.85) finally reads

$$
\frac{E}{P m}\left(\frac{\partial}{\partial \hat{t}}+\hat{\mathbf{u}} \cdot \hat{\nabla}\right) \hat{\mathbf{u}}=-\hat{\nabla} \hat{\Pi}^{*}-2 \mathbf{e}_{z} \times \hat{\mathbf{u}}+\operatorname{RaP} P \frac{\mathbf{r}}{r_{o}} \hat{T}+E \hat{\nabla}^{2} \hat{\mathbf{u}}+(\hat{\nabla} \times \hat{\mathbf{B}}) \times \hat{\mathbf{B}} .
$$

The non-dimensional set of partial differential equations constituted by the equation of motion (2.86), the evolution equation for the temperature perturbations (2.84) and the magnetic induction equation (2.80), supplemented by the fact that the flow is divergencefree in the Boussinesq approximation $(\hat{\nabla} \cdot \hat{\mathbf{u}}=0)$ and by the solenoidal magnetic field condition $(\hat{\nabla} \cdot \hat{\mathbf{B}}=0)$, can now be solved numerically upon the specification of opportune boundary conditions. The boundary conditions used in this study, as well as possible alternative choices, are discussed in the next section.

\subsection{Boundary conditions}

In this section, we specify appropriate boundary conditions for the set of MHD equations derived before. The fluid core is considered bounded in a spherical shell at the inner radius $r_{i}$ (corresponding to the ICB) and at the outer radius $r_{o}$ (corresponding to the $\mathrm{CMB}$ ). The overlying mantle is assumed to be electrically insulating while the inner core, mainly composed of iron, is modeled as a conductor. Figure 2.1 shows a sketch of the spherical shell geometry employed in the problem.

The Earth is actually an oblate spheroid due to the action of centrifugal forces. However, deviations from sphericity are small as a simple order of magnitude estimate suggests. Since the centrifugal acceleration $\mathbf{a}_{c}$ depends linearly on the distance from the rotation axis (see Section 2.4), it attains its maximum value at the equator of the planet where 


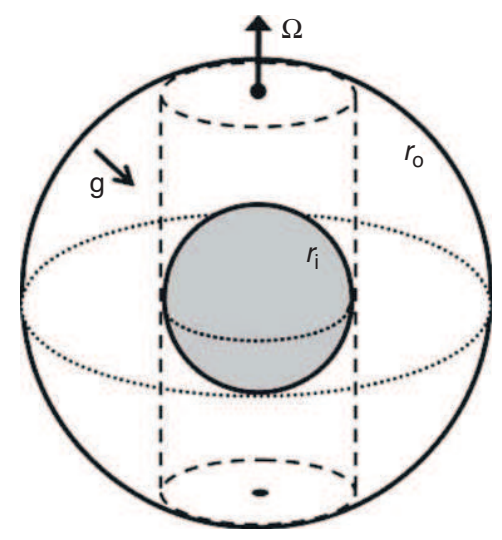

Figure 2.1: Sketch of the spherical shell geometry employed. The fluid shell is rotating about the $z$-axis with angular frequency $\Omega$. The inner core is highlighted in gray and broken lines mark the inner core tangent cylinder. From Christensen and Wicht (2007).

$\left|\mathbf{a}_{c}\right|=\Omega^{2} r_{E} \simeq 0.034 \mathrm{~m} \mathrm{~s}^{-2}$ ( $r_{E}$ being the Earth's radius). This corresponds approximately to $1 / 288$ of the gravitational acceleration at the Earth's surface. Spherical boundaries are therefore a good approximation when precession effects are not considered.

Having defined the fluid domain, we can now specify the boundary conditions for the velocity field $\mathbf{u}$, the magnetic field $\mathbf{B}$ and the temperature $T$. Firstly, we discuss the mechanical boundary conditions. Since the fluid is confined within the spherical shell, it cannot penetrate the inner and outer boundaries and the velocity field must therefore satisfy $\hat{\mathbf{e}}_{r} \cdot \mathbf{u}=0$, where $\hat{\mathbf{e}}_{r}$ is the unit vector in the radial direction. In the frame of reference co-rotating with the mantle, the fluid velocity is then assumed to vanish at the inner and outer boundaries, that is

$$
\mathbf{u}=\mathbf{0} \quad \text { at } r=r_{i}, r=r_{o} .
$$

These are referred to as rigid (or no-slip) mechanical boundary conditions. However, there is no a priori reason why the inner core should co-rotate with the mantle. A more appropriate mechanical boundary condition is then

$$
\mathbf{u}=\boldsymbol{\Omega}_{i} \times \mathbf{r} \text { at } r=r_{i},
$$

where $\boldsymbol{\Omega}_{i}=\Omega_{i} \hat{\mathbf{e}}_{z}$ is the inner core rotation rate. The change of rotation rate with respect to the mantle $d \Omega_{i} / d t$ is determined by the net torque acting on the conducting inner core. Gravitational, viscous and Lorentz forces contribute to such torque and an angular momentum equation must therefore be solved as part of the equation system. The no-slip condition at the ICB provides a relation between $\Omega_{i}$ and the toroidal velocity potential. The angular momentum equation can then be transformed simply to a matching condition for the toroidal velocity potential at $r=r_{i}$. For a complete mathematical formulation of the problem, as well as its numerical implementation, we refer to Wicht (2002). For simplicity, the inner core is assumed to have the same density $\rho$ and electrical conductivity $\sigma$ of the outer core.

Kuang and Bloxham (1997) argued that the use of rigid boundary conditions leads to large Ekman layer effects. The thickness of a viscous Ekman layer $\delta_{E}$ scales with the Ekman number as $\delta_{E} / \ell \sim \sqrt{E}$, where $\ell$ is a typical length scale of the system (Busse et al. 
2007). In the case of Earth's core $\delta_{E} \approx 10 \mathrm{~cm}$ and this possibly demonstrates the unimportant role of Ekman layers on the large scale flow dynamics and, consequently, on the magnetic field generation. In dynamo models, however, the Ekman number is at least 8-9 orders of magnitude larger than in the Earth's core and this leads to larger Ekman layers which might significantly influence the dynamo mechanism. In particular, helical fluid motions close to the rigid boundaries may contribute to the magnetic field production. The second type of mechanical boundary conditions often employed considers vanishing shear stresses. This is referred to as a free-slip boundary condition and is thought to represent more realistically the small viscous coupling between the core and the mantle. Despite such drawbacks, we use rigid inner and outer boundary conditions in this work.

We now discuss the magnetic boundary conditions. At the outer boundary, the continuity of the magnetic field reads

$$
\mathbf{B}=\mathbf{B}_{o} \quad \text { at } r=r_{o} .
$$

The mantle is assumed to be a perfect electrical insulator. This implies that no currents can flow in the mantle $\left(\mathbf{J}_{o}=\mathbf{0}\right)$ and therefore, from Ampère law $(2.64 \mathrm{c}), \nabla \times \mathbf{B}_{o}=\mathbf{0}$. This allows to write $\mathbf{B}_{o}$ as the gradient of a potential field $\phi\left(\mathbf{B}_{o}=-\nabla \phi\right)$. The solenoidal magnetic field condition finally gives $\nabla^{2} \phi=0$. This equation, together with the requirement that $\mathbf{B}_{o} \rightarrow \mathbf{0}$ as $r \rightarrow \infty$ (the source of $\mathbf{B}_{o}$ is in the core), completely specifies the magnetic field at the outer boundary.

Since the Earth's inner core is mainly composed of iron-nickel alloys, it can be modeled as a solid conducting sphere of radius $r=r_{i}$ with the same electrical conductivity $\sigma$ of the fluid shell. Therefore, a dynamo equation formally equivalent to (2.71) must be solved for the inner core. This equation is

$$
\frac{\partial \mathbf{B}_{i}}{\partial t}=\nabla \times\left(\mathbf{u}_{i} \times \mathbf{B}_{i}\right)+\eta \nabla^{2} \mathbf{B}_{i}
$$

where the velocity field $\mathbf{u}_{i}$ equals the solid body rotation of the inner core with respect to the reference frame of the mantle. The induction equations for the outer fluid shell (2.71) and for the inner core (2.89) have to be linked by two matching conditions. These conditions are the continuity of the magnetic field

$$
\mathbf{B}=\mathbf{B}_{i} \quad \text { at } r=r_{i}
$$

and the continuity of the horizontal electric field

$$
\mathbf{E}^{h}=\mathbf{E}_{i}^{h} \quad \text { at } r=r_{i},
$$

where the superscript ${ }^{h}$ denotes the horizontal component. Equation (2.91) can be readily written in terms of $\mathbf{B}$ and $\mathbf{B}_{i}$ using Ohm's law in the rotating frame of reference (2.67) and Maxwell's equation (2.64c).

Finally, we briefly examine the thermal boundary conditions commonly used in geodynamo models to drive core convection. The simplest assumption considers a fixed temperature contrast $\Delta T$ between the inner and outer boundaries:

$$
\begin{aligned}
& T=T_{o} \quad \text { at } r=r_{o}, \\
& T=T_{i} \quad \text { at } r=r_{i}
\end{aligned}
$$


where $\Delta T=T_{o}-T_{i}<0$. The lack of internal heat sources or sinks sets $h=0$ in the temperature equation $(2.55 \mathrm{c}$ ). Though there is no physical reason which justifies the conditions above, (2.92) are often assumed for simplicity.

Among the possible heat sources driving thermal convection in the Earth's core, the most important one is the latent heat released from the inner core solidification due to secular cooling (see Section 2.2). Secular cooling and internal heating are formally equivalent. The internal heat sources $h$ in the transport equation (2.55c) are generally considered homogeneously distributed in the fluid volume.

As the inner core freezes due to secular cooling, however, light elements cannot be dissolved efficiently anymore at the bottom of the fluid core. These light elements are likely buoyantly unstable and drive the so-called compositional convection. The compositional transport equation is formally equivalent to (2.55c) where $T$ has now to be intended as the perturbation concentration (from a mean reference state) of the light constituents of the core fluid. The constant release of light elements from the ICB can be modeled as a constant volumetric sink term $(h<0)$ in Equation (2.55c) (Kutzner and Christensen 2002). Since light elements cannot escape the outer core, an appropriate boundary condition at the CMB is a zero heat-flux. The latter two conditions are usually employed to model pure compositional convection.

The outer boundary condition can also take into account the fact that the mantle, having a finite thermal conductivity, controls the heat loss from the core. A fixed heat-flux condition at $r=r_{o}$ is generally employed in such cases. In addition, the net compositional flux from the inner boundary can be expected to depend directly from the heat loss to the mantle.

\subsection{The numerical method}

The non-dimensional equations derived in the previous sections and describing the dynamics of a convecting and conducting fluid in the Boussinesq approximation and in the presence of magnetic fields are

$$
\begin{aligned}
& \frac{E}{P m}\left(\frac{\partial}{\partial t}+\mathbf{u} \cdot \nabla\right) \mathbf{u}=-\nabla \Pi^{*}-2 \mathbf{e}_{z} \times \mathbf{u}+\operatorname{RaP} P \frac{\mathbf{r}}{r_{o}} T+E \nabla^{2} \mathbf{u}+(\nabla \times \mathbf{B}) \times \mathbf{B} \\
& \frac{\partial \mathbf{B}}{\partial t}=\nabla \times(\mathbf{u} \times \mathbf{B})+\nabla^{2} \mathbf{B} \\
& \frac{\partial T}{\partial t}+\mathbf{u} \cdot \nabla T=P m \nabla^{2} T .
\end{aligned}
$$

For simplicity, the superscripts ^ denoting non-dimensional quantities are suppressed in the above equations. Equations (2.93) are supplemented by the solenoidal velocity field condition $(\nabla \cdot \mathbf{u}=0)$ and by the solenoidal property of the magnetic field $(\nabla \cdot \mathbf{B}=0)$.

The above equations are solved numerically in a spherical shell which represents the Earth's outer core (Figure 2.1) and with the boundary conditions specified in Section 2.7. In this work we used the numerical implementation of Wicht (2002) which is based on developments from the code originally employed in early geodynamo simulations by Glatzmaier and Roberts (1995). In this section we briefly outline the basics of the pseudospectral method employed. Further details on the numerical method and the derivation of 
the spectral equations can be found in, e.g., Glatzmaier (1984), Glatzmaier and Roberts (1995), Wicht (2002) and Christensen and Wicht (2007).

The set of differential equations given above provides nine scalar equations in the eight unknowns $\mathbf{u}, \mathbf{B}, \Pi^{*}$ and $T$. The magnetic and velocity field components are not independent since both $\mathbf{u}$ and $\mathbf{B}$ are divergence-free. A convenient scalar decomposition of any solenoidal vector field is the well-known toroidal-poloidal decomposition (Chandrasekhar 1961, Appendix III). For the magnetic field $\mathbf{B}$ such decomposition reads

$$
\mathbf{B}=\nabla \times\left(\nabla \times g \frac{\mathbf{r}}{r}\right)+\nabla \times h \frac{\mathbf{r}}{r},
$$

where $g$ and $h$ are arbitrary functions of the position and time. The first and second terms on the RHS of the above equation are, respectively, the poloidal and toroidal fields. It is evident that (2.94) automatically guarantees that the divergence of $\mathbf{B}$ vanishes. An analogous equation defines the flow field by means of the poloidal and toroidal potentials $v$ and $w$ :

$$
\mathbf{u}=\nabla \times\left(\nabla \times v \frac{\mathbf{r}}{r}\right)+\nabla \times w \frac{\mathbf{r}}{r} .
$$

Under the toroidal-poloidal decomposition, the problem unknowns reduce to six scalar fields: the poloidal and toroidal potentials for the magnetic field $(g, h)$ and for the flow field $(v, w)$, the modified pressure $\Pi^{*}$ and the temperature perturbations $T$. Such fields are represented in a spherical system of coordinates $(r, \theta, \phi)$. A natural basis for the spectral representation of the unknown scalar fields in colatitude $\theta$ and longitude $\phi$ are the spherical harmonic functions

$$
Y_{\ell m}(\theta, \phi)=P_{\ell m}(\cos \theta) e^{i m \phi}
$$

where $\ell$ and $m$ denote degree and order respectively, and $P_{\ell m}$ are the associated Legendre functions. As an example, the poloidal field potential $g$ is then expanded as

$$
g(r, \theta, \phi)=\sum_{\ell=0}^{L} \sum_{m=-\ell}^{\ell} g_{\ell m}(r) Y_{\ell m}(\theta, \phi) .
$$

The radial variation of the unknowns is represented using Chebyshev polynomials. The Chebyshev polynomial of degree $n \in \mathbb{N}$ is defined by

$$
C_{n}(x)=\cos [n \arccos (x)]
$$

where $x \in[-1,1]$. The radial expansion of the poloidal magnetic potential in (2.97) truncated at degree $N$ reads

$$
g_{\ell m}(r)=\sum_{n=0}^{N} g_{\ell m n} C_{n}(r),
$$

where the coefficients $g_{\ell m n}$ have to be determined through the application of an inner product to the above expansion and using the orthogonality of Chebyshev polynomials. The expansion (2.99) is then evaluated numerically at specific radial grid points (collocation formulation). A particularly convenient choice of the $N_{r}$ collocation points is

$$
x_{k}=\cos \left(\pi \frac{k-1}{N_{r}-1}\right)
$$


with $k=1, \ldots, N_{r}$. The values of the Chebyshev polynomials at these points are, indeed, simply the cosine functions

$$
C_{n}\left(x_{k}\right)=\cos \left[n \frac{\pi(k-1)}{N_{r}-1}\right]
$$

The domain of Chebyshev polynomials $\left(-1 \leq x_{k} \leq 1\right)$ is transformed into the radial domain $\left(r_{i} \leq r_{k} \leq r_{o}\right)$ choosing

$$
x_{k}=2 \frac{r_{k}-r_{i}}{r_{o}-r_{i}}-1
$$

As a major advantage, this particular choice of collocation points provides the possibility to apply a fast Fourier transform between the physical and the spectral space. As a second benefit, the radial grid points are denser at the inner and outer boundaries which permits to better resolve the boundary layers.

The complete spectral representation of the poloidal field potential $g$ then reads

$$
g(r, \theta, \phi)=\sum_{\ell=0}^{L} \sum_{m=-\ell}^{\ell} \sum_{n=0}^{N} g_{\ell m n} C_{n}(r) Y_{\ell m}(\theta, \phi) .
$$

Since $g$ is a real-valued function, its spectral coefficients $g_{\ell m n}$ are not all independent but $g_{\ell, m, n}^{*}=g_{\ell,-m, n}$ with the superscript ${ }^{*}$ denoting complex conjugate. Only coefficients with $m \geq 0$ can be therefore considered in the expansion (2.103). Spectral expansions analogous to (2.103) hold for the remaining unknown scalar fields. The partial differential equations (2.93) separate then in (five) ordinary differential equations with respect to time in the (six) unknown spectral coefficients represented by the magnetic potentials $g_{\ell m n}$ and $h_{\ell m n}$, the velocity potentials $v_{\ell m n}$ and $w_{\ell m n}$, the pressure $\Pi_{\ell m n}^{*}$ and the temperature $T_{\ell m n}$.

Pressure $\Pi_{\ell m n}^{*}$, however, remains an additional unknown in the evolution equation for the poloidal flow potential. This problem can be solved in two ways. The first approach is to apply the curl operator to the Navier-Stokes equation 2.93a which yields to an evolution equation for the vorticity $\omega=\nabla \times \mathbf{u}$ without the pressure term. The second, more direct approach is to close the system deriving an equation involving the flow potentials and $\Pi_{\ell m n}^{*}$. This can be achieved by taking the horizontal divergence of the Navier-Stokes equation 2.93a (Christensen and Wicht 2007).

Several terms of the spectral equations (including the Coriolis force and the non-linear terms) couple modes in the spectral space and cause aliasing problems. To avoid such problems, the Coriolis force, the Lorentz force and the advection of momentum in (2.93a) are evaluated in the real space rather than in the spectral space. The same holds true for the source term in the induction equation (2.93b) and the temperature advection term in (2.93c). The spatial derivatives involved in the calculation of these terms are, however, evaluated in the spectral space. These terms are integrated explicitly in time using a second order Adam-Bashforth scheme. The remaining terms are implicitly time-stepped using a Crank-Nicolson scheme.

The numerical grid is defined in the code upon specification of the number of radial grid points $N_{r}$ and longitudinal grid points $N_{\phi} . N_{r}-1$ equals the maximum Chebyshev polynomial degree $N$ used in the expansion (2.103). In the numerical simulations analyzed in this work, we use $N_{r}=48$. The number of latitudinal grid points is adjusted 
to $N_{\theta}=N_{\phi} / 2$ to provide an isotropic resolution in the equatorial region. $N_{\phi}$ is related to the maximum spherical harmonic order $L$ in (2.103) by $L=2 / 3 N_{\theta}$. In our numerical simulations we use a number of longitudinal grid points $N_{\phi}$ up to 128 .

The time step $\Delta t$ is dynamically adapted during the temporal integration to fulfill the Courant-Friedrichs-Lewy (CFL) condition. The CFL condition demands that $\Delta t$ has to be smaller than the smallest advection time between two adjacent grid points

$$
\Delta t<\min \left(\frac{\Delta r}{u_{r}}, \frac{\Delta h}{u_{h}}\right)
$$

where $u_{r}$ and $u_{h}$ are the radial and horizontal characteristic velocities respectively, while $\Delta r$ and $\Delta h$ are the radial and horizontal grid intervals. The estimates of $u_{r}$ and $u_{h}$ account for both the fluid velocity and the local Alfvèn velocity. Further details on the time step control are given in Christensen et al. (1999). 


\section{Probability theory and statistical tools}

This chapter reviews some fundamentals of probability theory and presents a complete description of the statistical methods employed in the rest of this work. The basics of probability theory are discussed in Sections 3.1 3.3. The theoretical distributions and the associated stochastic processes tested by our statistical analysis are described in Section 3.4. Section 3.5 discusses the (frequentist) method we employed to estimate the unknown distribution parameters, while Section 3.6 describes the statistical tests used to determine the goodness-of-fit. Section 3.7 closes the chapter with a discussion on the problems of parameter inference and model selection in a Bayesian framework.

\subsection{The concept of probability}

It is not an easy task to define precisely and consistently the concept of probability. Different definitions of probability have indeed been developed in the past. Naively speaking, probability quantifies the degree of randomness of a certain property associated with a given system. In most practical situations it reflects our degree of uncertainty relative to the prediction of a certain event occurrence (e.g., the outcome of a measured physical quantity, the input parameters of a model describing observational data, etc.). The most direct definition of probability was formulated by Kolmogorov (1933) in the framework of set theory. In this section, we recall the Kolmogorov axioms of probability and we derive Bayes' theorem and the law of total probability.

Let $S$ be a set characterized by a certain number of elements (not yet explicitly specified) and called the sample space. We assign to each subset $A$ of $S$ a real number $\mathrm{P}(A)$, called the probability of $A$, which is defined by the following axioms:

1. for any $A \subset S, \mathrm{P}(A) \geq 0$;

2. for any disjoint subsets $A$ and $B(A \cap B=\emptyset)$, the probability of $A$ or $B$ is $\mathrm{P}(A \cup B)=$ $\mathrm{P}(A)+\mathrm{P}(B)$

3. the probability assigned to the sample space is $\mathrm{P}(S)=1$.

The above definition immediately implies $0 \leq \mathrm{P}(A) \leq 1$. Several basic properties of probability can be directly derived from these axioms (see, e.g., Cowan 1998, Chapter 1). 

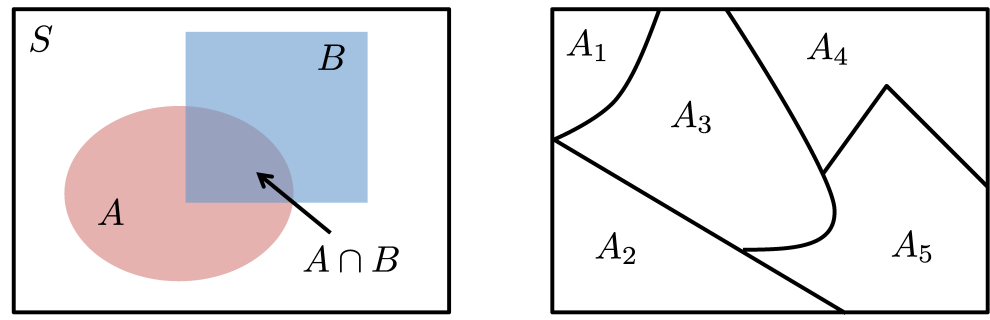

Figure 3.1: Left panel: Relationship between the sample space $S$ and the sets $A$ and $B$ in the definition of conditional probability. Right panel: Decomposition of the sample space $S$ into the disjoint sets $A_{i}, i=1, \ldots, 5$.

Consider the subsets $A \subset S$ and $B \subset S$ such that $\mathrm{P}(B) \neq 0$. The conditional probability $\mathrm{P}(A \mid B)$, namely the probability of $A$ given that $B$ is true, is defined as

$$
\mathrm{P}(A \mid B)=\frac{\mathrm{P}(A \cap B)}{\mathrm{P}(B)} .
$$

Figure 3.1 (left panel) illustrates the relation between $A$ and $B$ in the sample space $S$. The conditional probability (3.1) satisfies the Kolmogorov axioms of probability (Cowan 1998, Chapter 1).

Bayes' theorem naturally follows from the definition of conditional probability. Assuming $\mathrm{P}(A) \neq 0$, the conditional probability of $B$ given $A$ is

$$
\mathrm{P}(B \mid A)=\frac{\mathrm{P}(B \cap A)}{\mathrm{P}(A)} .
$$

Since $B \cap A=A \cap B$, the above definition yields

$$
\mathrm{P}(B \mid A) \mathrm{P}(A)=\mathrm{P}(A \cap B)=\mathrm{P}(A \mid B) \mathrm{P}(B),
$$

where (3.1) has been used in the last equality. The first and last equalities in (3.3) give

$$
\mathrm{P}(A \mid B)=\frac{\mathrm{P}(B \mid A) \mathrm{P}(A)}{\mathrm{P}(B)} .
$$

Equation (3.4) relates the conditional probabilities $\mathrm{P}(A \mid B)$ and $\mathrm{P}(B \mid A)$ and is known as the Bayes' theorem. We now derive the so-called law of total probability which will be useful in the following. Suppose the sample space $S$ to be divided into the disjoint subsets $A_{i}$, i.e. $S=\cup_{i=1}^{N} A_{i}$ with $A_{i} \cap A_{j}=\emptyset$ for any $i \neq j$, and assume that these subsets contain at least one element, that is $\mathrm{P}\left(A_{i}\right) \neq 0$ for all $i$. An illustration of this decomposition of the sample space is given in Figure 3.1 (right panel). Any arbitrary $B \subset S$ can then be written as

$$
B=B \cap S=B \cap\left(\cup_{i} A_{i}\right)=\cup_{i}\left(B \cap A_{i}\right) .
$$

Since the subsets $B \cap A_{i}$ are disjoint by construction (cf. Figure 3.1, right panel), the probability of $B$ is

$$
\mathrm{P}(B)=\mathrm{P}\left(\cup_{i}\left(B \cap A_{i}\right)\right)=\sum_{i} \mathrm{P}\left(B \cap A_{i}\right)
$$


and, using definition of conditional probability (3.1), we are lead to

$$
\mathrm{P}(B)=\sum_{i} \mathrm{P}\left(B \mid A_{i}\right) \mathrm{P}\left(A_{i}\right)
$$

Equation (3.5) is called the law of total probability and is useful when the sample space can be divided into subsets $A_{i}$ for which the probabilities are easy to calculate. Using the law of total probability in (3.4) yields to the equivalent formulation of Bayes' theorem

$$
\mathrm{P}(A \mid B)=\frac{\mathrm{P}(B \mid A) \mathrm{P}(A)}{\sum_{i} \mathrm{P}\left(B \mid A_{i}\right) \mathrm{P}\left(A_{i}\right)} .
$$

\subsubsection{Interpretation of probability}

The mathematical results discussed above are very general because they are valid for any function $\mathrm{P}$ which satisfies the Kolmogorov axioms. To apply these results in practical situations, we have to specify, firstly, the elements of the sample space where the probability is defined (i.e. the domain of P) and, secondly, a rule to assign to each element in the domain the respective probability value $\mathrm{P}$. At this stage, two interpretations of probability (the frequentist and the Bayesian) are commonly used. We describe these approaches in more detail below.

\subsubsection{Frequentist interpretation}

In the (classical) frequentist interpretation of probability, the elements of the sample space $S$ correspond to the possible outcomes of an experiment. In principle, the experiment has to be repeatable an infinite number of times under exactly the same conditions. A subset $A \subset S$ corresponds to the occurrence of any of the outcomes and is shortly referred to as an event. The probability of event $A$ is then defined as the fraction of times $A$ occurs in an infinite number of repeated experiments:

$$
\mathrm{P}(A)=\lim _{n \rightarrow \infty} \frac{n(A)}{n},
$$

where $n(A)$ is the number of experiments giving $A$ as an outcome and $n$ is the total number of experiments. In this interpretation the probability $\mathrm{P}$ is therefore the relative frequency of a given event. Hence, the conditional probability $\mathrm{P}(A \mid B)$ of Equation (3.1) is the number of cases where both $A$ and $B$ are observed divided by the number of occurrences of event $B$ alone.

\subsubsection{Bayesian interpretation}

The Bayesian interpretation of probability is more general and, in principle, contains the frequentist one. The subsets of the sample space $S$ are defined as hypotheses or propositions which can be either true or false. In this context, $S$ is often referred to as the hypothesis space rather than the sample space. The probability $\mathrm{P}(A)$ is then interpreted as

$$
\mathrm{P}(A)=\text { degree of belief that } A \text { is true. }
$$


To fulfill Kolmogorov axioms, the hypothesis space $S$ must be constructed such that only one of its elementary hypotheses is true.

The following example clarifies the role of probability in the Bayesian interpretation. Consider the situation in which we want to test the ability of a certain model $M$ to describe repeated measurements of a physical quantity (the data $D$ ) obtained from an experiment. The subset $A$ in Bayes' theorem (3.4) can then be interpreted as the proposition "model $M$ is true", while the subset $B$ is the proposition "the measurements $D$ are obtained from the experiment". Therefore, Bayes' theorem allow us to write

$$
\mathrm{P}(M \mid D) \propto \mathrm{P}(D \mid M) \mathrm{P}(M) .
$$

In the above expression $\mathrm{P}(M)$ represents the probability that model $M$ is true a priori, i.e. before conducting the experiment. For this reason, $\mathrm{P}(M)$ is commonly called the prior probability. The term $\mathrm{P}(D \mid M)$ on the RHS of (3.9) is the probability of obtaining the data $D$ given that model $M$ is true. In other words, $\mathrm{P}(D \mid M)$ quantifies how likely is the set of the observed measurements in the light of the model and is usually called the likelihood. Finally, the probability that model $M$ correctly describes the experimental data $D$ is given by $\mathrm{P}(M \mid D)$. This is called the posterior probability of the model in order to stress the fact that it is calculated after conducting the experiment. The normalization constant, dropped in Equation (3.9), is often referred to as the evidence because it depends on the data only.

The advantages of the Bayesian approach compared to the frequentist one are clear in the above example. In the first place, there is no limitation in the type of hypotheses which can be analyzed and Bayes' theorem applies to all of them. The intrinsic procedure of updating the prior probability assigned to a hypothesis is very similar to our natural learning process. Starting from an initial guess described by the prior, the information directly retrieved from experiments is used to construct our posterior knowledge. The likelihood indeed acts as a weight factor on the prior probability.

A possible weakness of the Bayesian approach may come from the fact that there is no objective way of calculating the prior probabilities. In the above example, the prior probability $\mathrm{P}(M)$ can be assigned according to the prior belief of the modeler himself on his theory. Reasonable choices of the prior probability have to be evaluated depending on the specific problem at hand. However, the prior probability can have only a minor impact on the posterior probability if, for example, the evidence coming from the data is strong. We will further discuss these issues in Section 3.7.

\subsection{Fundamentals of probability theory}

In this section, we review some fundamentals of classical statistical analysis and we introduce the notation employed in the rest of this work.

Consider an experiment whose outcome is described by a single continuos random variable (RV) $X$. The sample space $S$ introduced in Section 3.1 is then defined by the domain of $X$ and is often referred to as the statistical population. The experimental results, i.e. the repeated measurements of $X$, are regarded as the finite sample $\left(x_{1}, \ldots, x_{N}\right)$ which represents a set of $N>1$ random numbers drawn from the statistical population. 
Assume the probability that the RV $X$ does not exceed the value $x$, namely $\mathrm{P}(X \leq x)$, exists and is finite. This probability is called the cumulative distribution function (CDF) of $X$ and will be denoted as $F_{X}(x)$ hereafter. If $F_{X}(x)$ is a continuous non-decreasing function of $x$ differentiable everywhere (except, eventually, in a finite number of points), the probability density function $(\mathrm{PDF}) f_{X}(x)$ is

$$
f_{X}(x)=\frac{d F_{X}(x)}{d x} \geq 0 .
$$

Equivalently, the above definition reads

$$
F_{X}(x)=\int_{-\infty}^{x} f_{X}\left(x^{\prime}\right) d x^{\prime} .
$$

In its integral form, the PDF $f_{X}(x)$ is thus the probability of observing a value of the RV $X$ within the infinitesimal interval $[x, x+d x]$. In the frequentist interpretation of probability, $f_{X}(x)$ gives the fraction of times that the experimental outcome lies within $[x, x+d x]$ in the limit of an infinite number of observations. In order to fulfill the third Kolmogorov axiom (Section 3.1), the PDF $f_{X}(x)$ is normalized such that

$$
\int_{S} f_{X}(x) d x=1
$$

where the integration domain is the entire sample space $S$.

We now introduce two useful quantities which characterize the distribution of any random variable. The expectation value $\mathrm{E}_{X}[x]$ of a $\mathrm{RV} X$ distributed according to the PDF $f_{X}(x)$ is

$$
\mathrm{E}_{X}[x] \doteq \int_{-\infty}^{+\infty} x f_{X}(x) d x .
$$

The expectation value is also called the population mean and is generally shortly denoted by $\mu$.

The second quantity is the population variance $\mathrm{V}_{X}[x]$ and is defined by

$$
\mathrm{V}_{X}[x] \doteq \mathrm{E}_{X}\left[\left(x-\mathrm{E}_{X}[x]\right)^{2}\right]=\int_{-\infty}^{+\infty}(x-\mu)^{2} f_{X}(x) d x .
$$

The population variance (or simply variance) is shortly denoted by $\sigma^{2}$ and is a measure for the spread of $x$ about its mean value $\mu$. The definitions given in this section can be easily generalized to multi-dimensional random variables.

In the previous discussion, we adhered the common practice to denote the random variable in upper case and the actual value assumed by the corresponding lower case. For simplicity reasons, this notation might be sometimes avoided in the rest of this work; the distinction between random variables and their values should then be clear from the context.

\subsection{Survival and hazard functions}

In this section, we introduce the concepts of survival and hazard functions. They are used in the context of survival analysis, a branch of statistics dealing with the analysis of time durations to until a specified event happen. 
Let $T$ be a non-negative random variable which represents the time until an event occurrence. The probability that an event occurs by the time $t$ is the $\operatorname{CDF} F_{T}(t)=\mathrm{P}(T \leq t)$. It is convenient to define the complement of the $\mathrm{CDF}$

$$
S_{T}(t) \doteq \mathrm{P}(T>t)=\int_{t}^{\infty} f_{T}\left(t^{\prime}\right) d t^{\prime}=1-F_{T}(t)
$$

which is known as the survival function and represents the probability that the event of interest does not occur by the time $t$.

An alternative characterization of the distribution of $T$ is given by the instantaneous rate of occurrence of the event

$$
h_{T}(t) \doteq \lim _{d t \rightarrow 0} \frac{\mathrm{P}(t<T \leq t+d t \mid T>t)}{d t}
$$

which is called the hazard function. In the expression above, the numerator is the probability of observing an event in the infinitesimal time interval $(t, t+d t]$ provided that no event has occurred before time $t$. The value of such probability per unit time in the limit of infinitesimally small intervals gives then the instantaneous rate of occurrence of the event of interest. A relation between the hazard function $h_{T}(t)$ and the survival function $S_{T}(t)$ can be obtained using the definition of conditional probability (3.1) in (3.16)

$$
\begin{aligned}
h_{T}(t) & =\lim _{d t \rightarrow 0} \frac{\mathrm{P}(t<T \leq t+d t, T>t)}{\mathrm{P}(T>t) d t} \\
& =\lim _{d t \rightarrow 0} \frac{\mathrm{P}(t<T \leq t+d t)}{S_{T}(t) d t},
\end{aligned}
$$

where we used $\mathrm{P}(t<T \leq t+d t, T>t)=\mathrm{P}(t<T \leq t+d t)$ in the second equality. Since $\mathrm{P}(t<T \leq t+d t)=f_{T}(t) d t$, (3.17) reads

$$
h_{T}(t)=\frac{f_{T}(t)}{S_{T}(t)} .
$$

The expression above can also be intended as an alternative definition of the hazard function. Note that the hazard function is neither a probability nor a probability density.

By means of (3.10), Equation (3.18) can be written as

$$
h_{T}(t)=-\frac{d}{d t} \ln S_{T}(t)
$$

Integrating both sides of the above expression between 0 and $t$ gives

$$
\int_{0}^{t} h_{T}\left(t^{\prime}\right) d t^{\prime}=\ln S_{T}(0)-\ln S_{T}(t) .
$$

Assuming an event to be observed for $t>0, S_{T}(0)=1$ and the first term on the RHS of (3.20) equals zero. Using the definition of survival function (3.15), Equation (3.20) finally becomes

$$
F_{T}(t)=1-\exp \left[-\int_{0}^{t} h_{T}\left(t^{\prime}\right) d t^{\prime}\right] .
$$

This equation demonstrates that the hazard function uniquely identifies a distribution function. Statistical models can thus be constructed specifying the hazard rate only. In the next section we describe the distribution functions of interest for this work and we provide explicit expression for the respective hazard functions. 


\subsection{Statistical models and theoretical distributions}

The probability distributions of interest for our subsequent statistical analysis and the associated random processes are presented in Section 3.4.1. In this section particular attention is paid to modeling aspects of the geomagnetic reversal occurrences. Section 3.4.2 describes a particular type of random process known as the Poisson process. Further information on the wide variety of probability distributions and their properties can be found in, e.g., Forbes et al. (2011) and Johnson et al. (1995).

\subsubsection{Theoretical distributions}

\subsubsection{Uniform distribution}

A continuous $\mathrm{RV} X \in \mathbb{R}$ has a uniform distribution in the interval $[\alpha, \beta]$ if its PDF is

$$
f_{X}(x \mid \alpha, \beta)= \begin{cases}(\beta-\alpha)^{-1} & \text { for } \alpha \leq x \leq \beta \\ 0 & \text { otherwise. }\end{cases}
$$

Hereafter, we denote a uniform random variable by $X \sim \operatorname{Uniform}(\alpha, \beta)$. This distribution describes a quantity which is equally likely to be found between $\alpha$ and $\beta$. The mean and variance of a uniformly distributed RV are, respectively,

$$
\mathrm{E}_{X}[x]=\frac{1}{2}(\alpha+\beta)
$$

and

$$
\mathrm{V}_{X}[x]=\frac{1}{12}(\beta-\alpha)^{2} .
$$

The CDF of a uniform RV is then

$$
F_{X}(x \mid \alpha, \beta)= \begin{cases}0 & \text { for } x<\alpha \\ (x-\alpha) /(\beta-\alpha) & \text { for } x \in[\alpha, \beta) \\ 1 & \text { for } x \geq \beta\end{cases}
$$

Figure 3.2 shows the PDF and CDF of the (standard) uniform RV $X \sim \operatorname{Uniform}(0,1)$.

The uniform distribution is of fundamental importance in statistics because of the following property. The proof of this property can be found in any introductory textbook on classical statistical analysis. Let $X$ be a continuous RV with $\operatorname{CDF} F_{X}(x)$. The random variable $Y$ defined by the transformation

$$
y=F_{X}(x)
$$

is then uniformly distributed over the interval [0,1], that is $Y \sim \operatorname{Uniform}(0,1)$. Any RV can therefore be mapped into a standard uniform distribution by means of (3.26). This mapping is often called the probability-integral transformation and is particularly useful for practical purposes. Having a pseudo-random generator of uniformly distributed numbers, random deviates from any cumulative distribution can be obtained by a simple inversion of Equation (3.26). 

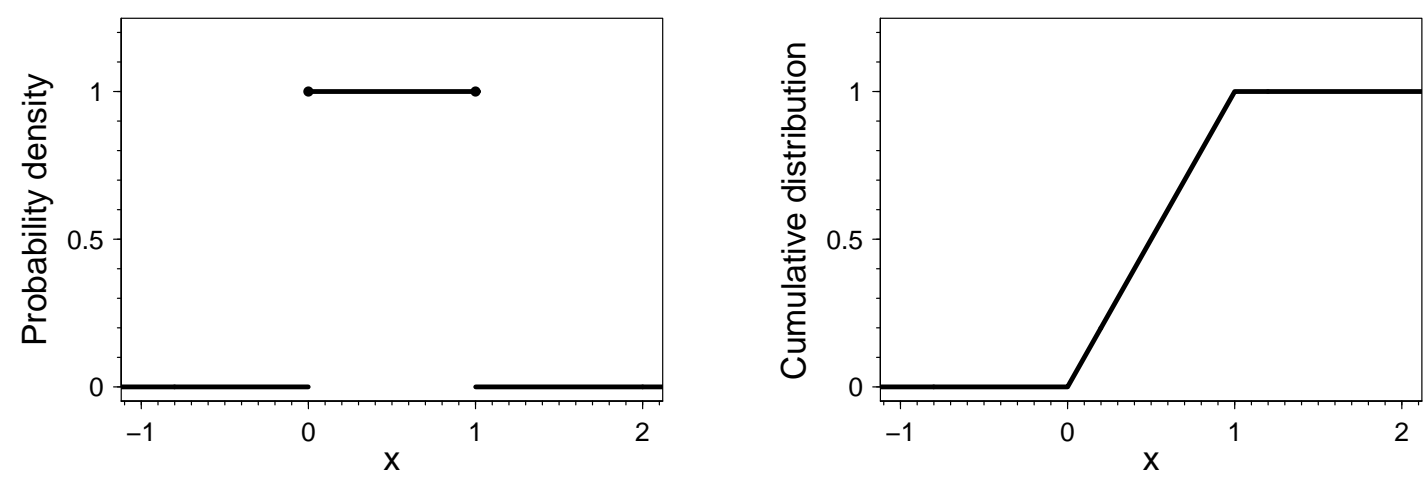

Figure 3.2: Probability density function (left panel) and cumulative distribution function (right panel) of a uniform random variable in the interval $[0,1]$.

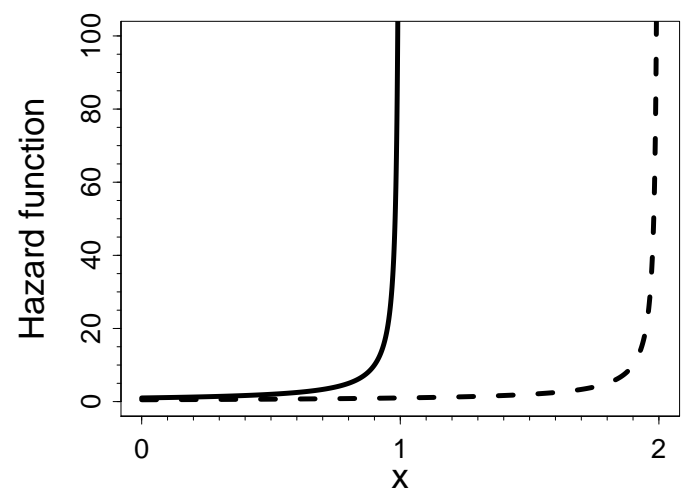

Figure 3.3: Hazard functions for the uniform distribution (3.27) within $[0,1]$ (solid line) and $[0,2]$ (dashed line).

The hazard function of a uniform distribution in the interval $[\alpha, \beta]$ is

$$
h_{X}(x)=\frac{1}{\beta-x}
$$

for $\alpha \leq x<\beta$. As an example, Figure 3.3 shows the hazard functions of Uniform $(0,1)$ and Uniform $(0,2)$. If $x$ is interpreted as the time period after the occurrence of a specified event, the rate of occurrence increases monotonically with time. In particular, $h_{X}$ is infinite for $x=\beta$ which means that, if an event did not occur for times $x<\beta$, an event will certainly happen at time $x=\beta$.

\subsubsection{Exponential distribution}

The non-negative continuous RV $X$ has an exponential distribution if its PDF is

$$
f_{X}(x \mid \lambda)=\lambda e^{-\lambda x},
$$

where $\lambda>0$ is known as the rate parameter. We denote an exponentially distributed RV by $X \sim \operatorname{Exp}(\lambda)$. The mean and variance of an exponentially distributed RV are, respectively,

$$
\mathrm{E}_{X}[x]=\lambda^{-1}
$$



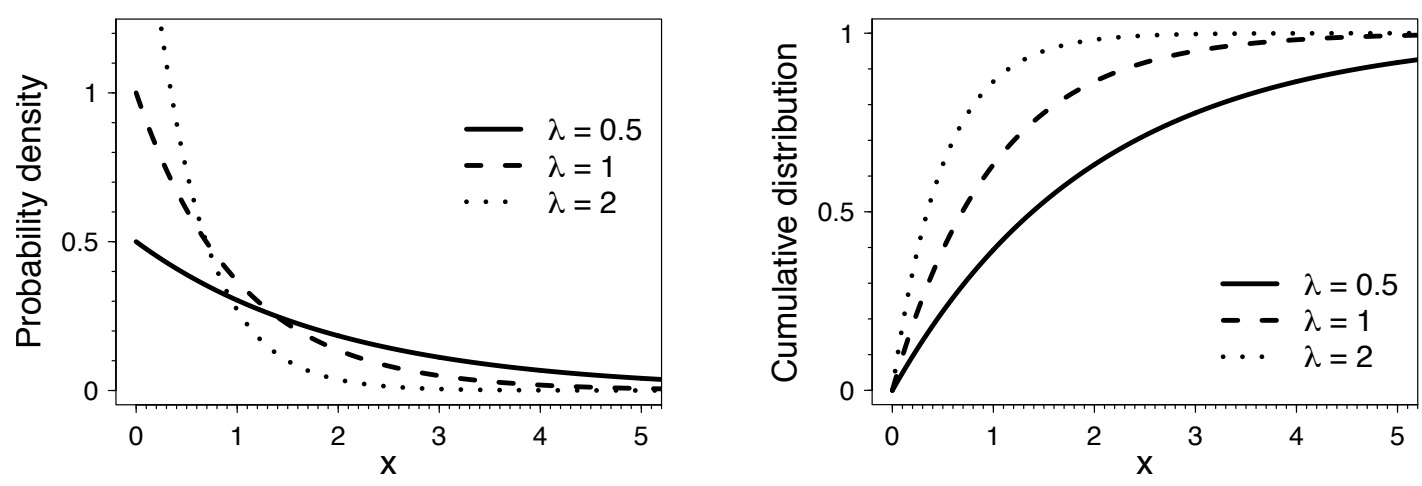

Figure 3.4: Probability density functions (left panel) and cumulative distribution functions (right panel) of an exponentially distributed random variable for various values of the rate parameter $\lambda$.

and

$$
\mathrm{V}_{X}[x]=\lambda^{-2}
$$

The exponential CDF is

$$
F_{X}(x \mid \lambda)=1-e^{-\lambda x}
$$

for $x \geq 0$. Figure 3.4 shows the exponential PDFs and CDFs for three different values of the rate parameter $\lambda$.

As will be discussed in detail in Section 3.4.2, the exponential distribution describes the inter-event times in a Poisson process. Cox (1968) suggested that the occurrence of geomagnetic reversals stems from a Poisson process. Analyzing one of the earliest reversal chronologies for the past $11 \mathrm{Myr}$ the author demonstrated that an exponential distribution well describes the observed chron durations. Two additional assumptions underly the Poisson hypothesis. First, events are assumed to occur instantaneously. Geomagnetic reversals, lasting on average at least one order of magnitude longer than polarity chrons, can be considered as instantaneous events in first approximation. The second assumption considers reversals occurring at a constant rate over the period spanned by the data.

The progressive improvement of magnetostratigraphic time scales, both in dating accuracy and recording of older events, revealed certain inconsistencies with the Poisson model. On time scales of tens of million years, the reversal occurrence might be influenced by mantle convection processes (see the discussion in Section 1.4.5). More recently, Constable (2000) reconstructed the reversal rate variations over the past 160 million years and found the reversal occurrence compatible with a non-stationary Poisson process.

The hazard function (3.18) for the exponential distribution is

$$
h_{X}(x)=\lambda \text {. }
$$

Since $h_{X}$ uniquely identifies a distribution function (Section 3.3), the exponential is the only distribution with a constant hazard rate. This means that the rate of occurrence of an event is independent of the time at which the last event happened. In other words, it is equally likely to find an event in any period of arbitrary length. The exponential distribution is thus said to be memoryless. A rigorous proof of such property will be given in Section 3.4.2 where the Poisson process is discussed in detail. 


\subsubsection{Gamma distribution}

The positive continuous RV $X$ has a gamma distribution, denoted by $X \sim \operatorname{Gamma}(k, \lambda)$ hereafter, if its PDF is

$$
f_{X}(x \mid k, \lambda)=\frac{\lambda^{k}}{\Gamma(k)} x^{k-1} e^{-\lambda x}
$$

with $k>0$ (shape parameter) and $\lambda>0$ (rate parameter) and where $\Gamma(k)$ is the gamma function evaluated at $k$ :

$$
\Gamma(k)=\int_{0}^{\infty} x^{k-1} e^{-x} d x
$$

Figure 3.5 shows gamma PDFs and CDFs for different values of the shape parameter $k$ and the rate parameter $\lambda$. Note that for $k=1$ the gamma reduces to an exponential distribution. For $k<1$, the gamma PDF is monotonically decreasing and drops faster than an exponential (Figure 3.5, upper left panel). For $k>1$, the PDF is skewed and bell-shaped (Figure 3.5, middle and lower left panels). When $k$ increases the distribution profile broadens and approaches a normal distribution for large $k$ values (typically for $k>10$ ). Increasing the rate parameter $\lambda$ tends to shrink the distribution function. This is quantified by the fact that, having $X \sim \operatorname{Gamma}(k, \lambda)$ and for any $c>0, c X \sim \operatorname{Gamma}(k, \lambda / c)$.

The mean and variance of a gamma distributed RV are, respectively,

$$
\mathrm{E}_{X}[x]=\frac{k}{\lambda}
$$

and

$$
\mathrm{V}_{X}[x]=\frac{k}{\lambda^{2}} .
$$

The gamma CDF is

$$
F_{X}(x \mid k, \lambda)=\frac{\gamma(k, \lambda x)}{\Gamma(k)}
$$

where $\gamma(k, \lambda x)$ is the lower incomplete gamma function

$$
\gamma(k, \lambda x)=\int_{0}^{\lambda x} t^{k-1} e^{-t} d t
$$

Cumulative distributions (3.36) are shown in the right panels of Figure 3.5 for various values of the distribution parameters.

Substituting (3.33) and (3.36) into (3.18) yields to the hazard function of the gamma distribution

$$
h_{X}(x)=\frac{\lambda^{k} x^{k-1} e^{-\lambda x}}{\Gamma(k)-\gamma(k, \lambda x)} .
$$

Figure 3.6 illustrates the gamma hazard functions for $\lambda=1$ and for different values of the shape parameter $k$. The constant hazard $h_{X}=1$ is obtained for $k=1$ where the gamma reduces to an exponential distribution with $\lambda=1$ (see Section 3.4.1.2). For $k<1$ the hazard function decreases monotonically to the asymptotic value $\lambda$. If the random variable describes the time until a specified event happens, the occurrence is initially encouraged for $k<1$. If $k>1$, the hazard function increases monotonically to the asymptotic value $\lambda$. This corresponds to a diminished likelihood of an event occurrence for a certain period. 

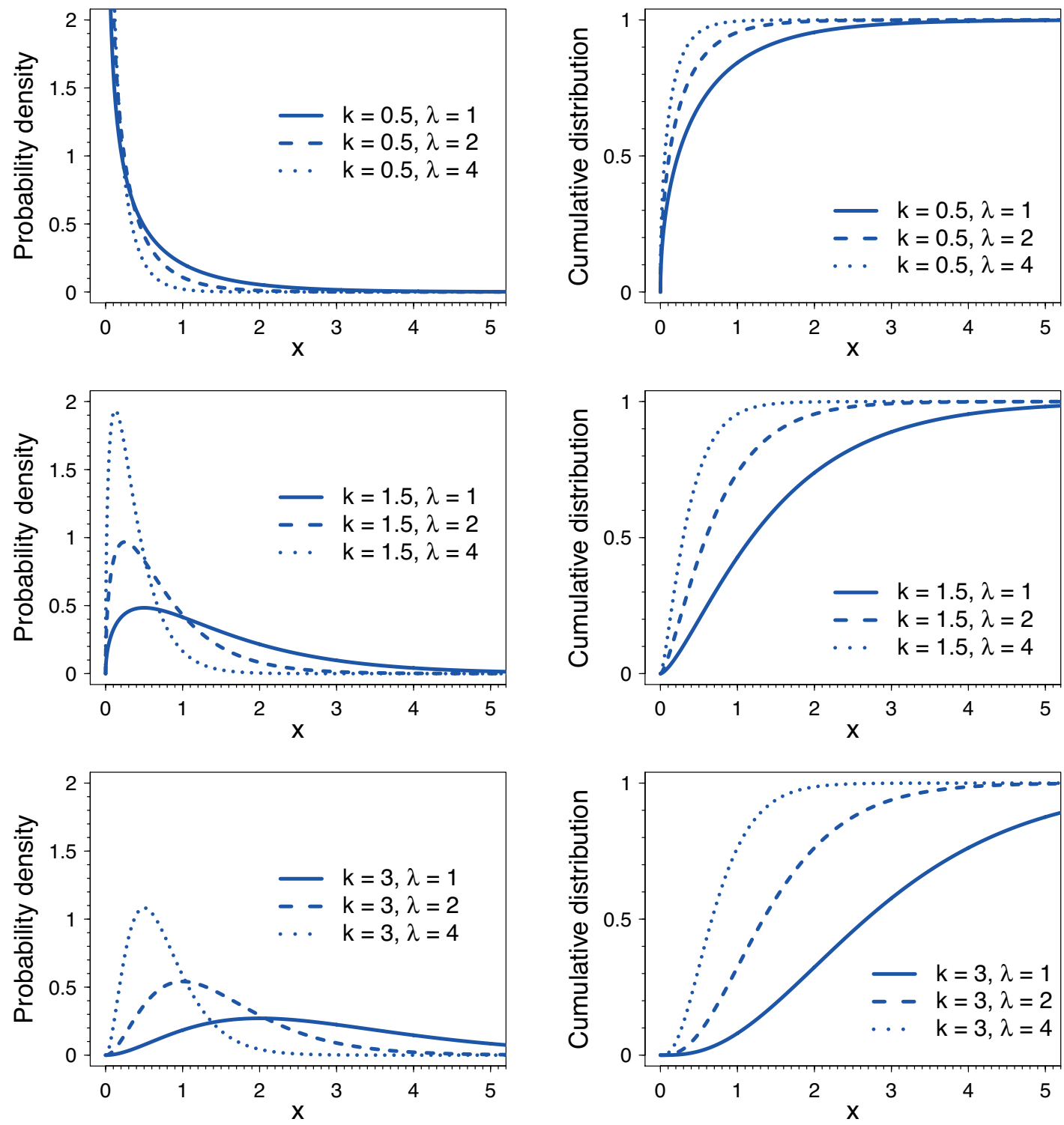

Figure 3.5: Gamma probability density functions (left panels) and cumulative distribution functions (right panels) for various values of the shape parameter $k$ and the rate parameter $\lambda$. Upper, middle and lower rows show $k=0.5, k=1.5$ and $k=3$ respectively. Each plot presents three different values of the rate parameter, namely $\lambda=1, \lambda=2$ and $\lambda=4$. 
The larger $k$ the longer this inhibition period is (cf. Figure 3.6). If this inhibition period is left, events tend to occur almost with a constant hazard $\lambda$.

Analyzing a reversal chronology for the past 48 million years, Naidu (1971) was the first who demonstrated that paleomagnetic chrons can be well described by a gamma distribution with $k>1$.

As already discussed in Section 1.4.4, geomagnetic reversal chronologies suffer of two major problems: the accurate dating of certain individual events and a reliable record of the shorter polarity intervals. The censoring of brief chrons (typically shorter than $30 \mathrm{kyr}$ ) represents a gross error in the sequence of polarity intervals as highlighted by the following example. Consider a sequence of three intervals of normal, reverse and normal (NRN) polarities. If the R interval is short and it is not resolved, the sequence is then (incorrectly) identified as a single long lasting $\mathrm{N}$ interval. This censoring effect relates with the following property of the gamma distribution. Let $t_{1}, \ldots, t_{n}$ be $n$ independent observations from an exponential distribution with rate $\lambda$. If such observations are concatenated into the single interval

$$
T_{n}=\sum_{i=1}^{n} t_{i},
$$

then $T_{n}$ is gamma distributed with shape $k=n$ and rate $\lambda$. This property constitutes a link between the exponential and gamma distributions and a proof can be found in McFadden (1984). Therefore, if short chrons in paleomagnetic reversal chronologies are missed, the filtered sequence of polarity intervals results compatible with a gamma distribution with relatively large values of $k$.

Simulating the unfiltered sequence of polarity intervals by adding few short chrons to the reversal chronology for the past $65 \mathrm{Myr}$ and accounting for the non-stationarity of the reversal occurrences, McFadden and Merrill (1984) estimated a gamma shape parameter $k$ significantly biased towards low values. The authors demonstrated that there is a significant evidence for $k=1$ in the unfiltered sequence of chrons, in agreement with a Poisson occurrence of reversals.

More recently, Marzocchi (1997) studied the influence of missing intervals on synthetic time series of events. The author suggested that the number of short polarity inter-

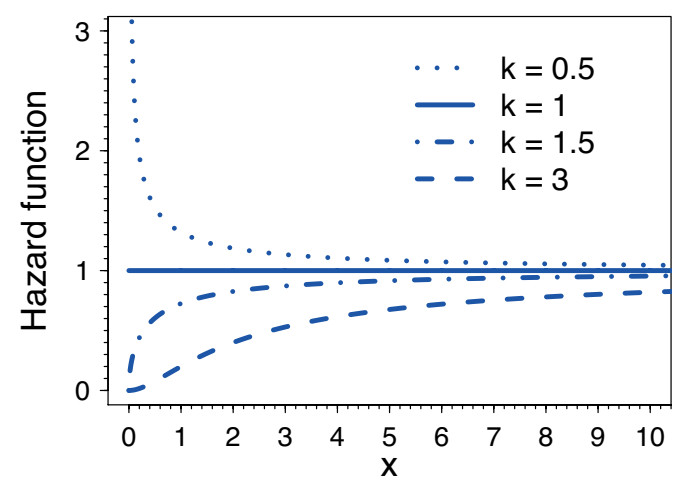

Figure 3.6: Hazard functions for the gamma distribution (3.37) with $\lambda=1$ and four different values of $k$ as shown in the legend. The constant solid line refers to $k=1$ where the gamma reduces to an exponential distribution. Other values of $k$ are the same of those presented in the PDF plots of Figure 3.5 . 
vals $(<30 \mathrm{kyr})$ missed in a purely Poisson process which accounts for the value of the gamma shape parameter $k$ estimated from paleomagnetic reversal chronologies amounts to about $35 \%$ of the original events number.

McFadden and Merrill (1993) proposed an alternative explanation for the observed deviations from the Poisson model relying on the intrinsic inability of the core to generate new reversals for a short period immediately following a polarity transition. Assuming that paleomagnetic chrons are gamma distributed, the authors estimated this period to last about $45 \mathrm{kyr}$ which roughly matches the time required by the dipole field to diffuse in the Earth's core by ohmic processes.

A comprehensive review of gamma models used to describe certain characteristics of the geomagnetic reversal occurrences can be found in McFadden (1984).

\subsubsection{Weibull distribution}

The Weibull distribution is an extension of the exponential distribution and has been named after Weibull (1951). This distribution is defined quite naturally from a power transformation of the exponential. The positive RV $X$ has a Weibull distribution if

$$
Y=(\lambda X)^{s},
$$

where $\lambda>0$ and $s>0$, has the (standard) exponential distribution

$$
f_{Y}(y)=e^{-y} .
$$

The Weibull PDF can be obtained applying the change of variables rule to (3.39) and reads

$$
f_{X}(x \mid s, \lambda)=s \lambda^{s} x^{s-1} e^{-(\lambda x)^{s}},
$$

where $s$ and $\lambda$ are called the shape and rate parameters respectively. The Weibull CDF is

$$
F_{X}(x \mid s, \lambda)=1-e^{-(\lambda x)^{s}} .
$$

We denote a Weibull distributed random variable by $X \sim$ Weibull $(s, \lambda)$ hereafter.

Figure 3.7 shows the Weibull PDFs (left panels) and CDFs (right panels) for different values of the shape and rate parameters. The PDFs decrease monotonically for $s<1$ (Figure 3.7, upper left panel), while is bell-shaped for $s>1$. Similarly to the gamma, the Weibull distribution reduces to an exponential with rate $\lambda$ for $s=1$, namely Weibull $(1, \lambda)=\operatorname{Exp}(\lambda)$.

The Weibull hazard function, obtained substituting (3.41) and (3.42) into (3.18), is

$$
h_{X}(x)=s \lambda^{s} x^{s-1} \text {. }
$$

Figure 3.8 presents the hazard function (3.43) for $\lambda=1$ and different values of the shape parameter $s$. This function is monotonically decreasing for $s<1$ and equals $\lambda$ for $s=1$ as expected for an exponential distribution (Equation (3.32) ). On the contrary, $h_{X}$ increases monotonically for $s>1$. The case $s=2$ is of particular interest because provides a linear variation in the hazard rate. If the variable $x$ is interpreted as the waiting time until the occurrence of a certain event, a Weibull distribution with $s>1$ predicts the event occurrence to become more likely with time. This situation cannot be described using, for example, a gamma distribution which tends to a constant hazard rate as $x \rightarrow \infty$ (cf. Figure 3.6). 

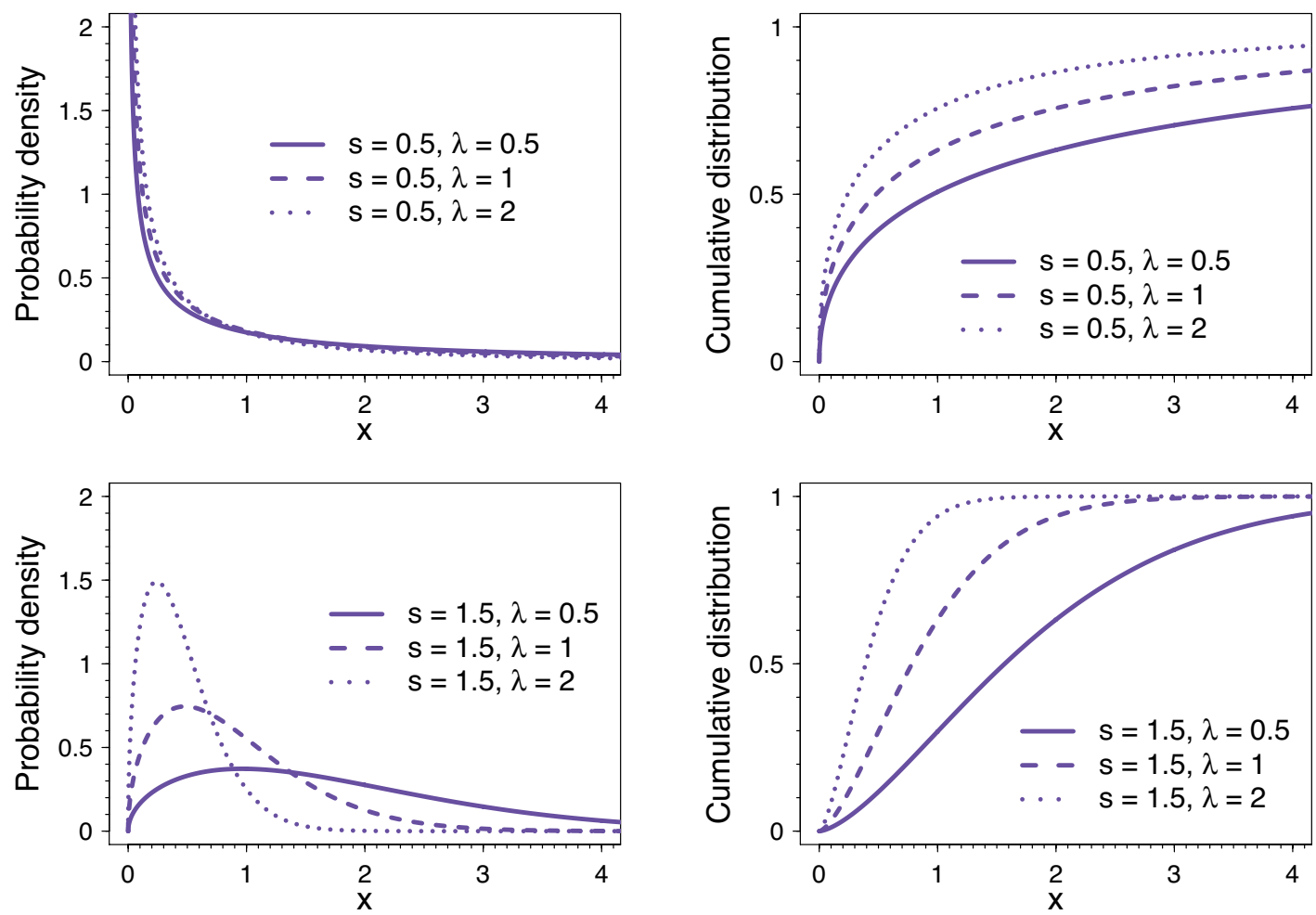

Figure 3.7: Weibull probability density functions (left panels) and cumulative distribution functions (right panels) for various values of the shape parameter $s$ and the rate parameter $\lambda$ (see legend insets).

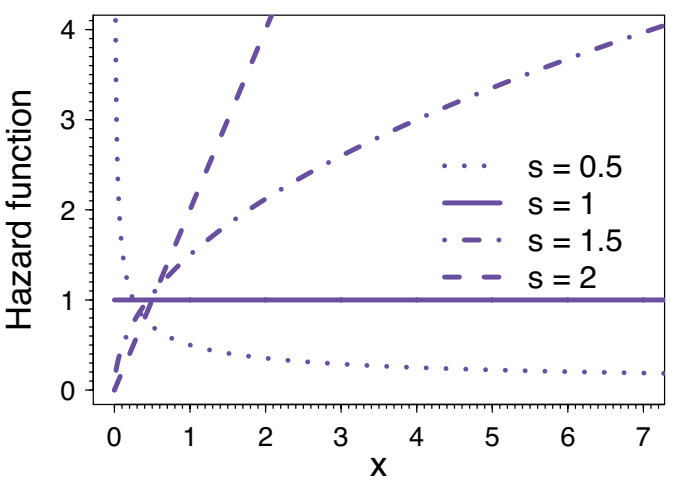

Figure 3.8: Hazard functions of the Weibull distribution (3.43) with rate $\lambda=1$ and four different values of the shape parameter $s$ as given in the legend. The (constant) solid line refers to $s=1$ where the Weibull distribution reduces to the exponential $\operatorname{Exp}(1)$. 


\subsubsection{Log-normal distribution}

The log-normal distribution, as its name suggests, can be introduced as a logarithmic transformation of a normally distributed random variable. Let $X$ be a positive RV such that

$$
Y=\ln X
$$

has a normal distribution with mean $\mu \in \mathbb{R}$ and standard deviation $\sigma>0$, denoted as $Y \sim \operatorname{Normal}(\mu, \sigma)$ with PDF

$$
f_{Y}(y \mid \mu, \sigma)=\frac{1}{\sqrt{2 \pi} \sigma} \exp \left[-\frac{(y-\mu)^{2}}{2 \sigma^{2}}\right] .
$$

The RV $X$ is said to be $\log$-normally distributed and we write $X \sim \operatorname{LogNormal}(\mu, \sigma)$ hereafter. Applying the change of variables rule to (3.45), we obtain the log-normal PDF of $X$

$$
f_{X}(x \mid \mu, \sigma)=\frac{1}{\sqrt{2 \pi} \sigma x} \exp \left[-\frac{(\ln x-\mu)^{2}}{2 \sigma^{2}}\right],
$$

where $x>0$. An alternative parameterization is obtained considering the transformation $\mu=\ln m$, where $m>0$. The PDF (3.46) then reads

$$
f_{X}(x \mid m, \sigma)=\frac{1}{\sqrt{2 \pi} \sigma x} \exp \left[-\frac{[\ln (x / m)]^{2}}{2 \sigma^{2}}\right]
$$

and $m$ coincides with the geometric mean (and also with the median) of the distribution. The CDF of $X \sim \operatorname{LogNormal}(\mu, \sigma)$ is

$$
F_{X}(x \mid \mu, \sigma)=\frac{1}{2}\left[1+\operatorname{erf}\left(\frac{\ln x-\mu}{\sigma \sqrt{2}}\right)\right]
$$

where erf is the error function.

Mean and variance of a log-normally distributed RV are, respectively,

$$
\mathrm{E}_{X}[x]=e^{\mu+\frac{1}{2} \sigma^{2}}
$$

and

$$
\mathrm{V}_{X}[x]=\left(e^{\sigma^{2}}-1\right) e^{2 \mu+\sigma^{2}}=\left(e^{\sigma^{2}}-1\right)\left(\mathrm{E}_{X}[x]\right)^{2} .
$$

The above expressions can be readily obtained from (3.44) recalling that $\mathrm{E}_{Y}[y]=\mu$ and $\mathrm{V}_{Y}[y]=\sigma^{2}$. Figure 3.9 plots the log-normal probability densities and cumulative distributions for $\mu=0$ and different values of the standard deviation $\sigma$.

The hazard function, obtained substituting (3.46) and (3.48) into (3.18), is

$$
h_{X}(x)=\frac{\exp \left[-\frac{(\ln x-\mu)^{2}}{2 \sigma^{2}}\right]}{\sqrt{\frac{\pi}{2}} \sigma x\left[1-\operatorname{erf}\left(\frac{\ln x-\mu}{\sigma \sqrt{2}}\right)\right]} .
$$

Figure 3.10 illustrates the hazard function (3.51) for $\mu=0$ and the three different $\sigma$ values shown in Figure 3.9. These functions present an initial increase followed by a monotonic decrease. If the random variable $X$ is interpreted as the waiting time until a specified 

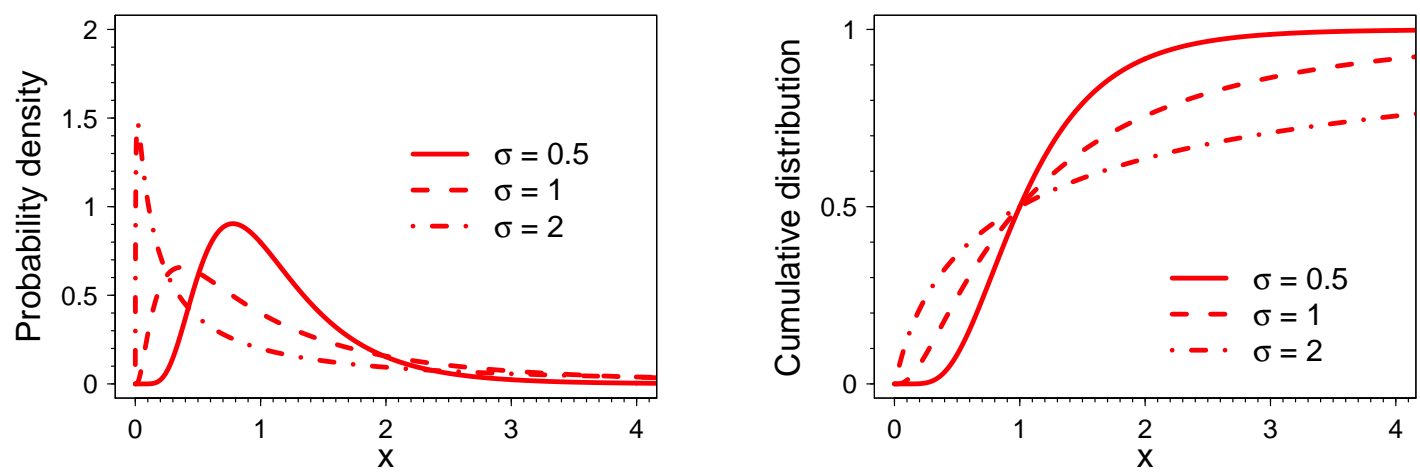

Figure 3.9: Log-normal probability density functions (left panel) and cumulative distribution functions (right panel) with location parameter $\mu=0$ and various values of $\sigma$ (see legend inset).

event happens, the event occurrence is initially encouraged while becomes progressively less likely with time. This naturally favors long waiting times leading to PDFs with pronounced tails. The larger $\sigma$ the lower the asymptotic value of $h_{X}$ is; higher $\sigma$ values thus correspond to heavier distribution tails (cf. Figure 3.9).

We now discuss an interesting property of the log-normal distribution. Let $\left\{X_{i}\right\}$ be a set of $n$ (positive) independent and identically distributed RVs and define the product

$$
Z_{n}=\prod_{i=1}^{n} X_{i}
$$

which equivalently reads

$$
\ln Z_{n}=\sum_{i=1}^{n} \ln X_{i} .
$$

As $n \rightarrow \infty$, the central limit theorem states that the RV $\ln Z_{n}$ is normally distributed with mean $\mu=\mathrm{E}_{Z_{n}}\left[\ln z_{n}\right]$ and variance $\sigma^{2}=\mathrm{V}_{Z_{n}}\left[\ln z_{n}\right]$. This demonstrates that the limiting distribution of $Z_{n}$ is a log-normal.

The property above highlights the fact that the log-normal distribution often arises in processes involving the multiplicative contribution of random effects (multiplicative noise). Hoyng et al. (2001), for example, proposed a mean-field dynamo model which involves a multiplicative noise mechanism and well reproduces certain characteristics of the geomagnetic dipole field variability. The authors suggested that multiplicative noise effects arise in the random helicity fluctuations originating in the turbulent core convection. These random fluctuations are parametrized in the $\alpha$-term of the mean-field equation and multiply over time when time-stepping the induction equation.

Ryan and Sarson (2007) investigated similar stochastic processes and proposed a reversing mean-field $\alpha \omega$-dynamo coupled with a shell model of turbulence. The authors demonstrated that the simulated polarity intervals, similarly to paleomagnetic chrons, are well-described by a log-normal distribution. This heavy-tailed distribution naturally accounts for superchrons. These extreme events might therefore stem from the same internal random processes triggering shorter polarity intervals (see also the discussion in Section 1.4.5). 


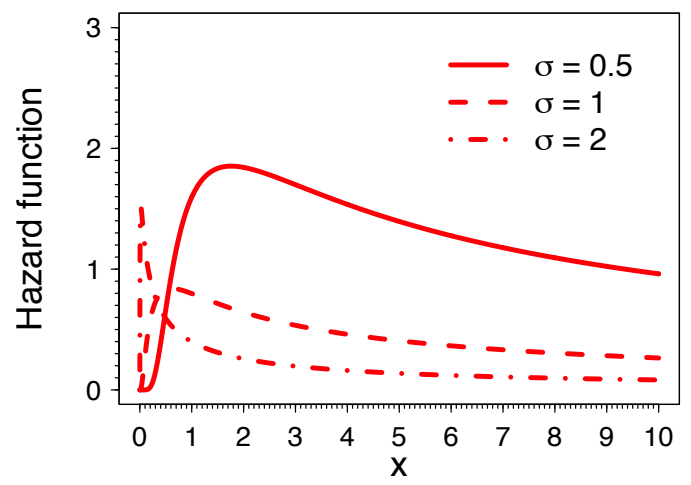

Figure 3.10: Hazard functions for the log-normal distribution (3.51) with location parameter $\mu=0$ and various $\sigma$ values as given in the legend.

\subsubsection{Log-logistic distribution}

A positive random variable $X$ has a log-logistic distribution if its PDF is

$$
f_{X}(x \mid \alpha, \theta)=\frac{\alpha(x / \theta)^{\alpha-1}}{\theta\left[1+(x / \theta)^{\alpha}\right]^{2}},
$$

where $\alpha>0$ and $\theta>0$ are the shape and scale parameters respectively. Hereafter, we refer to a $\log$-logistically distributed random variable to as $X \sim \log \operatorname{Logistic}(\alpha, \theta)$. The $\log$-logistic CDF reads

$$
F_{X}(x \mid \alpha, \theta)=\frac{1}{1+(x / \theta)^{-\alpha}} .
$$

Figure 3.11 illustrates the log-logistic PDFs and CDFs for $\theta=1$ and different $\alpha$ values. If $\alpha<1$, the PDF is a monotonic decreasing function. If $\alpha>1$, the PDF is unimodal with mode $\theta[(\alpha-1) /(\alpha+1)]^{1 / \alpha}$.

The log-logistic hazard function, obtained by means of (3.18), is

$$
h_{X}(x)=\frac{\alpha(\alpha / \theta)^{\alpha-1}}{\theta\left[1+(x / \theta)^{\alpha}\right]} .
$$
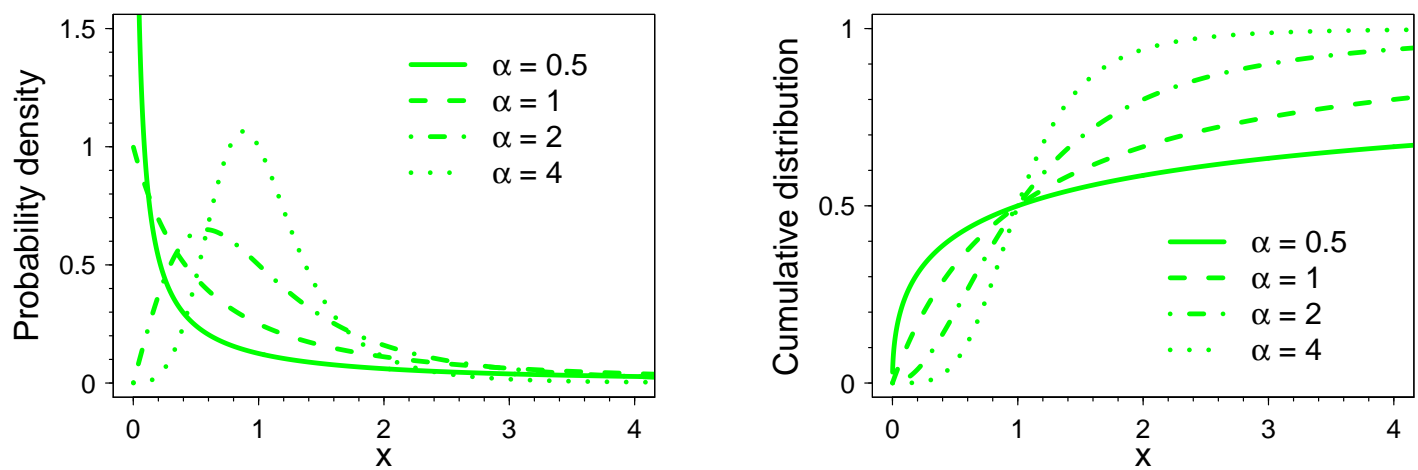

Figure 3.11: Log-logistic probability density functions (left panel) and cumulative distribution functions (right panel) for $\theta=1$ and different values of the shape parameter $\alpha$ (see the legend insets). 


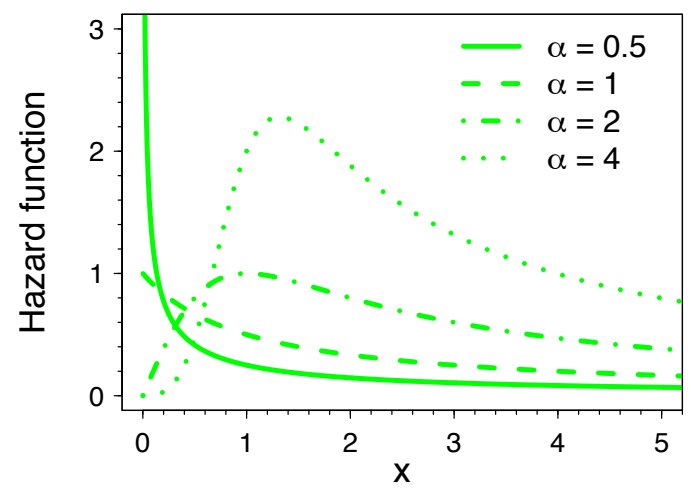

Figure 3.12: Hazard functions of the log-logistic distribution (3.56) for a scale parameter $\theta=1$ and various values of the shape parameter $\alpha$ as given in the legend.

Figure 3.12 plots log-logistic hazard functions for $\theta=1$ and different values of $\alpha$ which correspond to those shown in Figure 3.11. The hazard function (3.56) decreases monotonically for $\alpha \leq 1$. If $x$ is interpreted as the temporal interval until a specified event occurs, the likelihood of observing an event decreases with time. For $\alpha>1$, the hazard rate increases for relatively short waiting times and decreases monotonically afterwards. A similar behavior also characterizes the log-normal distribution (see Section 3.4.1.5).

\subsubsection{The Poisson process}

The Poisson process is a stochastic process for modeling the temporal occurrence of certain events in a system. Many natural phenomena (e.g., the radioactive decay of atoms or the occurrence of earthquakes) and a large variety of daily-life situations (e.g., the incoming telephone calls to a help line or the customer purchases at a store) are welldescribed as Poisson processes. Cox (1968) was the first who modeled the occurrence of geomagnetic reversals in terms of a Poisson process. In the following, we formally define a Poisson process and we derive the distribution of the inter-event times in such a process and its memoryless property.

The Poisson process can be formally defined as a counting process in which the events (or arrivals) occur continuously and independently. Let $N(t)$ be the number of events which occur during the time interval $(0, t]$. We define $N(0)=0$ because no events can occur before the counting starts. The number of arrivals in any time interval $h>0$ is $N(t+h)-N(t)$ and is called the increment.

The (homogeneous) Poisson process of rate $\lambda>0$ is defined as the infinite collection of random variables $\{N(t)\}$ (obtained for each $t>0$ ) with the following properties:

(i) the increments in disjoint time intervals are independent;

(ii) the increments are stationary;

(iii) the events occur rarely, i.e.

$$
\begin{aligned}
& \mathrm{P}(N(h)=0)=1-\lambda h+O\left(h^{2}\right), \\
& \mathrm{P}(N(h)=1)=\lambda h+O\left(h^{2}\right)
\end{aligned}
$$

as $h \rightarrow 0$. 
The first property states that the arrivals in any time interval $(0, t]$ do not affect the events occurrence in any later period. In other word, for any time interval $h>0, N(t+h)-N(t)$ and $N(t)$ are mutually independent random variables. The second property states that the distribution of $N(t+h)-N(t)$ is the same for each $h>0$, i.e. is independent of $t$. Intuitively, this means that the events are equally likely to occur at all times. The third property regards the probability of observing a single event in an infinitely small time interval and equals $\lambda h$ if higher order terms are neglected. Similarly, the probability for zero arrivals is $1-\lambda h$. The probability for two or more events is then

$$
1-\mathrm{P}(N(t)=0)-\mathrm{P}(N(t)=1) \sim O\left(h^{2}\right)
$$

and can therefore be neglected to the first order.

We now calculate the probability distribution of $N(t)$, namely $\mathrm{P}(N(t))$, under the above assumptions. Consider the interval $[0, t]$ where $k$ arrivals are observed. We discretize such period into $n$ sub-intervals of equal lengths $\delta \ll 1$. Let $N\left(t_{i}\right), i=0, \ldots, n-1$ be the number of arrivals in each sub-interval. As discussed above, the probability of more than two arrivals during any sub-interval is $O\left(h^{2}\right)$ and can thus be neglected to the first order.

In any sub-interval, we observe either a success (a single event with probability $\mathrm{P}\left(N\left(t_{i}\right)-\right.$ $\left.N\left(t_{i-1}\right)\right)=1$ ) or a failure (no event and therefore $\mathrm{P}\left(N\left(t_{i}\right)-N\left(t_{i-1}\right)\right)=0$ ). Since the increments $N\left(t_{i}\right)-N\left(t_{i-1}\right)$ are independent by definition, this process is equivalent to a sequence of $n$ Bernoulli trials (e.g., the binary outcomes of $n$ independent coin tosses) with success probability $p=\lambda \delta$ at each trial. The probability of observing $k$ successes in $n$ Bernoulli trials is (see, e.g., Cowan 1998)

$$
\mathrm{P}(N(t)=k)=\left(\begin{array}{l}
n \\
k
\end{array}\right) p^{k} q^{n-k},
$$

where $q=1-p$ is the failure probability and

$$
\left(\begin{array}{l}
n \\
k
\end{array}\right)=\frac{n !}{k !(n-k) !}
$$

is the binomial coefficient which describes the number of $k$-combinations in a set of $n$ elements. If $t$ is kept fixed, the approximation (3.58) becomes more precise as $\delta \rightarrow 0$. In this case, $n=t / \delta \rightarrow \infty$ but the expected number of successes $n p$ remains finite and equals $\lambda t$.

Under these circumstances, the binomial probability (3.58) is approximated by the Poisson distribution with parameter $\lambda t$ (see, e.g., Cowan 1998)

$$
\lim _{n \rightarrow \infty} \mathrm{P}(N(t)=k) \simeq \frac{(\lambda t)^{k}}{k !} e^{-\lambda t}, \quad \text { with } k=0,1, \ldots .
$$

Note that, if $\lambda t \ll 1$, a Taylor expansion of $e^{-\lambda t}$ in the above expression demonstrates that the above property (iii) is fulfilled by (3.60).

\subsubsection{Distribution of waiting times and memoryless property}

In this section, we demonstrate that the inter-event times in a Poisson process are exponentially distributed. Consider a (homogeneous) Poisson process $\{N(t) ; t>0\}$ with rate 
$\lambda>0$ and let $T_{k}$ be the $k$-th arrival time $(k=1,2, \ldots)$. If $T_{k}>t$, it is clear that the number of arrivals until time $t$ is smaller than $k$ and therefore

$$
\mathrm{P}\left(T_{k}>t\right)=\mathrm{P}(N(t)<k) .
$$

We now consider the time until the first arrival $T_{1}$. According to the above equation, $T_{1}$ is greater than $t$ only if no events occurred before $t$ and therefore

$$
\begin{aligned}
\mathrm{P}\left(T_{1}>t\right) & =\mathrm{P}(N(t)=0) \\
& =\frac{(\lambda t)^{0}}{0 !} e^{-\lambda t}=e^{-\lambda t},
\end{aligned}
$$

where we used the Poisson distribution (3.60) for $k=0$ events. The cumulative distribution function of $T_{1}$ is

$$
F_{T_{1}}(t) \doteq \mathrm{P}\left(T_{1} \leq t\right)=1-\mathrm{P}\left(T_{1}>t\right)=1-e^{-\lambda t} .
$$

Equation (3.10) gives the PDF of $T_{1}$

$$
f_{T_{1}}(t)=\lambda e^{-\lambda t}
$$

which demonstrates that the waiting time until the first arrival is exponentially distributed. Using the assumption of independent increments, it can be shown that all the following inter-arrival times $T_{k}-T_{k-1}$ are exponentially distributed. Hence, the waiting times $X_{k}=$ $T_{k}-T_{k-1}$ in a Poisson process are independent and identically distributed random variables with $X_{k} \sim \operatorname{Exp}(\lambda)$.

Among all types of arrival-processes, the Poisson process is the only memoryless one. A stochastic process is said to be memoryless if

$$
\mathrm{P}(X>t+x \mid X>t)=\mathrm{P}(X>x)
$$

for any $t \geq 0$. If the RV $X$ is interpreted as the waiting time until a certain arrival, the above expression states that, provided there is no arrival before time $t$, the probability for an event occurring at $x>t$ equals the probability of the total waiting time $x$. Intuitively, this means that an event occurrence is not influenced by the previous history.

As it shown in the following, the above property (3.64) holds for an exponential distribution (i.e. for the waiting times $X \sim \operatorname{Exp}(\lambda)$ in a Poisson process). Using the definition of conditional probability (3.1), the RHS of (3.64) can be written as

$$
\begin{aligned}
\mathrm{P}(X>t+x \mid X>t) & =\frac{\mathrm{P}(X>t+x, X>t)}{\mathrm{P}(X>t)} \\
& =\frac{\mathrm{P}(X>t+x)}{\mathrm{P}(X>t)}
\end{aligned}
$$

where the last equality descends from the fact that the event $\{X>t+x\}$ necessarily implies $\{X>t\}$. By means of the CDF (3.62), the above expression becomes

$$
\frac{\mathrm{P}(X>t+x)}{\mathrm{P}(X>t)}=\frac{e^{-\lambda(t+x)}}{e^{-\lambda t}}=\mathrm{P}(X>x)
$$

which finally demonstrates the validity of (3.64). 


\subsection{The method of maximum likelihood}

Consider the RV $X$ with PDF $f_{X}(x \mid \boldsymbol{\theta})$ where $\boldsymbol{\theta}=\left(\theta_{1}, \ldots, \theta_{M}\right)$ are its $M$ free parameters. Suppose to have $N$ repeated measurements of $X$ yielding to the observations $\mathbf{x}=$ $\left(x_{1}, \ldots, x_{N}\right)$. In the following we describe the method of maximum likelihood (ML), a technique for estimating the parameters $\boldsymbol{\theta}$ given the sample of observed values $\mathbf{x}$.

Assuming the measurements to be independent and identically distributed, the probability of observing the sample $\mathbf{x}$ is

$$
\mathcal{L}(\boldsymbol{\theta})=\prod_{i=1}^{N} f_{X}\left(x_{i} \mid \boldsymbol{\theta}\right) .
$$

$\mathcal{L}(\boldsymbol{\theta})$ measures how likely the observations $\mathbf{x}$ are and is therefore called the likelihood function. The maximum likelihood method defines the estimators $\hat{\boldsymbol{\theta}}=\left(\hat{\theta}_{1}, \ldots, \hat{\theta}_{M}\right)$ of the unknown parameters $\boldsymbol{\theta}$ as those values which maximize the likelihood function:

$$
\hat{\boldsymbol{\theta}}=\max _{\boldsymbol{\theta}} \mathcal{L}(\boldsymbol{\theta})
$$

and therefore requiring the data to be most likely. It is useful to define the log-likelihood function $\ell(\boldsymbol{\theta})$ as

$$
\ell(\boldsymbol{\theta}) \doteq \ln \mathcal{L}(\boldsymbol{\theta})=\sum_{i=1}^{N} \ln f_{X}\left(x_{i} \mid \boldsymbol{\theta}\right)
$$

such that the ML estimators $\hat{\boldsymbol{\theta}}$ are given by the solutions of the equations

$$
\frac{\partial \ell}{\partial \theta_{j}}=0, j=1, \ldots, M
$$

Note that the ML method assumes the validity of the model $f_{X}(\mathbf{x} \mid \boldsymbol{\theta})$ in describing the data. The choice of a poor or wrong model usually manifests in a likelihood function which spreads over a large range of parameter values. The main advantage of the ML method compared to other parameter estimation techniques is that no data binning is required.

As an example, we apply the ML method to estimate the rate parameter $\lambda$ of the exponential distribution (Section 3.4.1.2). Using the exponential PDF (3.28), the loglikelihood function (3.67) reads

$$
\ell(\lambda)=\sum_{i=1}^{N}\left(\ln \lambda-\lambda x_{i}\right)=N \ln \lambda-\lambda \sum_{i=1}^{N} x_{i} .
$$

Maximizing $\ell(\lambda)$ gives the ML estimator

$$
\hat{\imath}=\frac{N}{\sum_{i=1}^{N} x_{i}}
$$

which coincides with the inverse of the sample mean as expected.

Figure 3.13 illustrates this parameter estimation problem with a numerical experiment. A sample of 50 random deviates has been drawn from an exponential distribution with rate $\lambda=1$. The ML estimate (3.70) is $\hat{\lambda}=1.197$ and the corresponding exponential 

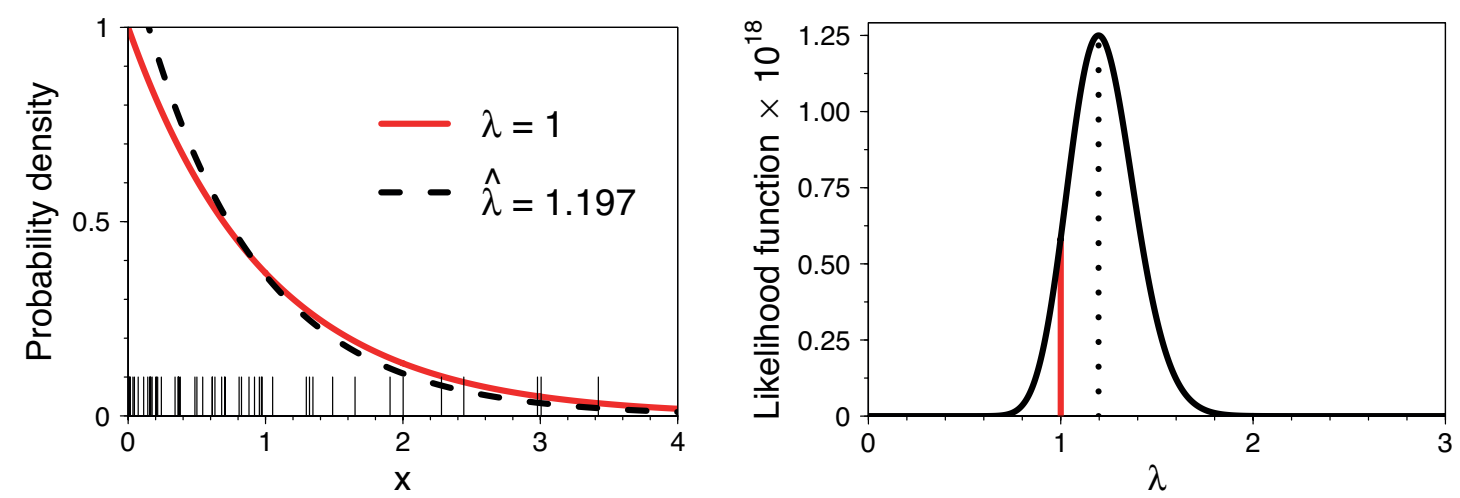

Figure 3.13: Maximum likelihood estimate of the exponential rate parameter $\lambda$ in a numerical experiment. The random sample has been drawn from an exponential distribution with $\lambda=1$ and is constituted by $N=50$ observations (tick marks on the horizontal axis of the left panel). Left panel: Exponential probability density evaluated with the true rate $\lambda=1$ (red curve) and with the maximum likelihood estimate $\hat{\lambda}$ (black curve). Right panel: Likelihood function of the sample (3.65) with the vertical lines denoting the true rate (red) and the estimate $\hat{\lambda}$ (black).

PDF closely resembles the expected profile (Figure 3.13 , left panel). The estimate $\hat{\lambda}$ does not exactly match the true value due to the poor sample size and the inherent random fluctuations. The right panel of Figure 3.13 presents the likelihood function $\mathcal{L}(\lambda)$ obtained from (3.69). Increasing the number of observations tends to peak the likelihood function around the true value as will be discussed in the next sections.

The ML method, however, does not necessarily guarantee a well-defined estimator. The properties which characterize an optimal estimator will be presented in Section 3.5.2.

\subsubsection{Transformation invariance of the ML estimator}

An important property of the ML estimator is its invariance under variable transformations. We demonstrate this property in this section. Consider the generic functional transformation $a(\theta)$ of the parameter $\theta$ and its ML estimate $\hat{\theta}$ to be known. The ML estimate of $a$ results from the solution of

$$
\frac{\partial \ell}{\partial a}=\frac{\partial \ell}{\partial \theta} \frac{\partial \theta}{\partial a}=0 \text {. }
$$

The condition $\partial_{a} \ell=0$ in the equation above is satisfied if $\partial_{\theta} \ell=0$ and thus $\theta=\hat{\theta}$. Therefore, the evaluation of $a$ at $\hat{\theta}$ gives the ML estimate of $a$

$$
\hat{a}=a(\hat{\theta})
$$

which demonstrates the invariance of the ML estimator $\hat{\theta}$. Note that the properties of the estimator $\hat{a}$ are, however, not necessarily the same of $\hat{\theta}$.

\subsubsection{Desirable properties of estimators}

The definition of an estimator for a certain quantity, for what reasonable may seem, is rather arbitrary and certainly not unique. Here we describe the three properties which 
qualify the "goodness" of an estimator. For simplicity, the following discussion is limited to a scalar parameter $\theta$ but it can be readily extended to multiple dimensions.

\subsubsection{Consistency}

An estimator $\hat{\theta}$ is said to be consistent if it converges to the true parameter value $\theta$ as the sample size $N$ increases, that is

$$
\lim _{N \rightarrow \infty} \hat{\theta}=\theta
$$

This is the fundamental property an estimator has to fulfill. For example, the ML estimator of the exponential rate parameter (3.70) converges to the true value $\lambda$ in the limit of an infinite number of observations due to the law of large numbers.

\subsubsection{Bias}

The bias $b$ of an estimator $\hat{\theta}$ is defined as the difference between its expectation value and the true value $\theta$, namely

$$
b=\mathrm{E}_{\theta}[\hat{\theta}]-\theta \text {. }
$$

In some cases the bias $b$ can be calculated analytically. As an example, consider the exponential distribution and the ML estimator of its rate parameter (3.70). The expectation value of $\hat{\lambda}$ is

$$
\begin{aligned}
\mathrm{E}_{\lambda}[\hat{\lambda}] & =\int \hat{\lambda} f_{X}(\mathbf{x} \mid \lambda) d \mathbf{x} \\
& =\lambda \frac{N}{N-1},
\end{aligned}
$$

where $f_{X}(\mathbf{x} \mid \lambda)$ is the joint exponential PDF of the data sample $\mathbf{x}$ assuming that the measurements are independent and identically distributed. Substituting the expectation value above in (3.74) yields to the bias of the rate parameter

$$
b=\frac{\lambda}{N-1} .
$$

The above expression shows that the ML estimator $\hat{\lambda}$ is unbiased only in the limit $N \rightarrow \infty$ (asymptotically unbiased).

Since it is not always possible to calculate analytically the bias (3.74), a numerical approach is often employed. To obtain the expectation value $\mathrm{E}_{\theta}[\hat{\theta}]$, a set of $M \gg 1$ experiments are simulated numerically. Each experiment consists of $N$ random deviates drawn from the given probability density $f_{X}(x \mid \theta)$, where $\theta$ is the true distribution parameter which is kept fixed. The estimate of the distribution parameter varies for each experiment due to the inherent statistical fluctuations. We therefore obtain the set of values $\left\{\hat{\theta}_{1}, \ldots, \hat{\theta}_{M}\right\}$ which is representative of the distribution of the original estimate $\hat{\lambda}$. The expectation value $\mathrm{E}_{\theta}[\hat{\theta}]$ is then calculated as the sample mean of the ensemble and the convergence of the estimator to the true parameter value can be finally studied. 


\subsubsection{Efficiency, estimator variance and the Cramèr-Rao bound}

The third property relates with the statistical uncertainty of an estimator. A natural measure of this uncertainty is the estimator variance

$$
\mathrm{V}_{\theta}[\hat{\theta}]=\mathrm{E}_{\theta}\left[\hat{\theta}^{2}\right]-\left(\mathrm{E}_{\theta}[\hat{\theta}]\right)^{2}
$$

An estimator $\hat{\theta}_{1}$ is said to be more efficient than $\hat{\theta}_{2}$ if $\mathrm{V}_{\theta_{1}}\left[\hat{\theta}_{1}\right]<\mathrm{V}_{\theta_{2}}\left[\hat{\theta}_{2}\right]$.

In the example of the exponential distribution, the variance of the ML estimator of the rate parameter $\hat{\lambda}$ is

$$
\begin{aligned}
\mathrm{V}_{\lambda}[\hat{\lambda}] & =\mathrm{E}_{\lambda}\left[\hat{\lambda}^{2}\right]-\left(\mathrm{E}_{\lambda}[\hat{\lambda}]\right)^{2} \\
& =\int \hat{\lambda}^{2} f_{X}(\mathbf{x} \mid \lambda) d \mathbf{x}-\left[\int \hat{\lambda} f_{X}(\mathbf{x} \mid \lambda) d \mathbf{x}\right]^{2} \\
& =\frac{\lambda^{2}}{N},
\end{aligned}
$$

where $f_{X}(\mathbf{x} \mid \lambda)$ is the joint exponential PDF of the observations $\mathbf{x}$. In the last equality we used the fact that the measurements are independent and identically distributed. Note that the variance (3.78) is a function of the true (and unknown) rate parameter $\lambda$. To obtain an estimate for the statistical error of $\hat{\lambda}$, the ML invariance (Section 3.5.1) has to be used. According to (3.72), the ML estimate of the variance $\hat{V}_{\hat{\lambda}}$ can thus be obtained replacing $\lambda$ in (3.78) with its ML estimator $\hat{\lambda}$ and therefore $\hat{V}_{\hat{\lambda}}=\hat{\lambda}^{2} / N$. Since it can be shown, under fairly general conditions, that in the large sample limit the likelihood function (3.65) is normally distributed (see Section 3.5.3), the interval $\hat{\lambda} \pm\left(\hat{V}_{\hat{\lambda}}\right)^{1 / 2}$ coincides with the $68.3 \%$ confidence interval of the estimate.

However, it is not always possible to solve analytically the integrals in (3.77). Moreover, the analytical solution becomes impractical if the selected model is characterized by a large number of free parameters. Numerical calculations similar to those discussed in the previous section for the estimator bias are then employed.

It is reasonable to suppose that no estimator having zero variance can be constructed. This limitation is a natural consequence of the fact that any estimator is a function of the data sample. The data sample comes indeed from repeated observations of the same quantity which are subject to random fluctuations inherent in the measurement process itself. In other words, any parameter estimate will be always known with a certain "error" and the variance (3.77) provides a measure for this degree of uncertainty. In this context, a fundamental result is the Cramèr-Rao inequality

$$
\mathrm{V}_{\theta}[\hat{\theta}] \geq \frac{\left(1+\frac{\partial b}{\partial \theta}\right)^{2}}{\mathcal{I}(\theta)}
$$

where $\mathcal{I}(\theta)$ is the Fisher information

$$
\mathcal{I}(\theta) \doteq \mathrm{E}_{\theta}\left[\left(\frac{\partial}{\partial \theta} \ln f_{X}(\mathbf{x} \mid \theta)\right)^{2}\right] .
$$

The Cramèr-Rao inequality (3.79) sets a lower bound for the variance of any estimator $\hat{\theta}$. A proof of (3.79) can be found in Brandt (1997). Note that biased estimators $(b \neq 0)$ have 
a larger variance than unbiased ones. This is expected because biased estimators diverge from the true parameter value $\theta$ already by construction. If the equality in (3.79) holds, the estimator $\hat{\theta}$ has the minimum variance and is said to be efficient.

If the measurements $x_{1}, \ldots, x_{n}$ are independent, the Fisher information (3.80) can be written as

$$
\begin{aligned}
\mathcal{I}(\theta) & =-\mathrm{E}_{\theta}\left[\frac{\partial^{2} \ln f_{X}(\mathbf{x} \mid \theta)}{\partial \theta^{2}}\right] \\
& =-\mathrm{E}_{\theta}\left[\frac{\partial^{2} \ell(\theta)}{\partial \theta^{2}}\right] .
\end{aligned}
$$

Intuitively, the fact that $\mathcal{I}(\theta)$ is proportional to the second derivative of the log-likelihood $\ell(\theta)$ measures how fast the convergence of the estimator to the true value is in the parameter space. The term "information" used for $\mathcal{I}(\theta)$ is justified by the fact that a peaked likelihood is more informative because the range of significant parameter values is narrower.

In the example of the exponential distribution, the RHS of the Cramèr-Rao inequality for the ML estimator of the rate parameter $\hat{\lambda}$ equals the variance (3.78) obtained from the analytical calculation. The estimator of the rate parameter (3.70) is therefore an efficient estimator.

A simpler expression for the lower bound of the variance of a generic unbiased $(b=0)$ ML estimator can be obtained using (3.81). By means of the transformation invariance (3.72), the lower bound for $\hat{V}_{\hat{\theta}}$ is given by (3.79) evaluating the second derivative in (3.81) at the ML estimate $\hat{\theta}$ :

$$
\hat{V}_{\hat{\theta}}=\left[-\left.\frac{\partial^{2} \ell(\theta)}{\partial \theta^{2}}\right|_{\theta=\hat{\theta}}\right]^{-1} \text {. }
$$

The above expression is particularly useful because it does not involve any additional analytical and/or numerical calculations. This result will be used in our subsequent statistical analysis to evaluate the statistical error associated with a certain parameter estimate. The estimate (3.82) can be easily generalized to multidimensional parameter estimates.

\subsubsection{Asymptotic behavior of the ML estimator}

In this section we derive the distribution of the ML estimator in the limit of an infinite number of observations $N$. For simplicity, we consider the case of a single parameter estimate $\theta$. We start our derivation expanding the log-likelihood function (3.67) in a Taylor series about the ML estimate $\hat{\theta}$ :

$$
\ell(\theta)=\ell(\hat{\theta})+\left.\frac{\partial \ell}{\partial \theta}\right|_{\theta=\hat{\theta}}(\theta-\hat{\theta})+\left.\frac{1}{2} \frac{\partial^{2} \ell}{\partial \theta^{2}}\right|_{\theta=\hat{\theta}}(\theta-\hat{\theta})^{2}+\ldots
$$

The first term on the RHS of the above expression is the log-likelihood maximum, while the second term is zero by definition. For an efficient and unbiased estimator we can write

$$
\frac{\partial^{2} \ell}{\partial \theta^{2}} \simeq \mathrm{E}_{\theta}\left[\frac{\partial^{2} \ell}{\partial \theta^{2}}\right]=-\frac{1}{\mathrm{~V}_{\theta}[\hat{\theta}]},
$$


where we used (3.82). In the limit of an infinite data sample, the third and higher order terms in (3.83) can be ignored because $\ell(\theta)$ strongly peaks at $\hat{\theta}$ and we are thus lead to

$$
\ell(\theta) \simeq \ell(\hat{\theta})-\frac{(\theta-\hat{\theta})^{2}}{2 \mathrm{~V}_{\theta}[\hat{\theta}]} .
$$

The likelihood function consequently reads

$$
\mathcal{L}(\theta)=\mathcal{L}(\hat{\theta}) \exp \left(-\frac{(\theta-\hat{\theta})^{2}}{2 \mathrm{~V}_{\theta}[\hat{\theta}]}\right)
$$

which finally shows that $\mathcal{L}(\theta)$ is asymptotically normal. In the case of multiple parameters, the asymptotic limit of the likelihood function is a multivariate normal distribution and the derivation is equivalent to that given above.

\subsection{Goodness-of-fit tests}

Statistical tests are used to quantify how well the observed data agree with the predicted probabilities from a given model or with other features characterizing a certain population. The hypothesis under test is called the null hypothesis and is conventionally denoted as $H_{0}$. If $H_{0}$ specifies a probability distribution which represents the model assumed to describe the data, the statistical test is called a goodness-of-fit test. Goodness-of-fit tests rely on a statistic explicitly constructed to quantify the agreement between the observed measurements and the predictions of $H_{0}$. If the distribution under $H_{0}$ is completely specified (i.e. all the free model parameters are known) the hypothesis is said to be simple. In most practical situations, however, the unknown parameters are estimated from the data themselves (using, for example, the maximum likelihood method described in Section 3.5). The null hypothesis is said to be composite in this case.

In the following sections we introduce two statistical tests designed for null hypotheses concerning continuous distributions, the Kolmogorov-Smirnov and Anderson-Darling goodness-of-fit tests. These tests compare the observed cumulative distributions with the cumulative probabilities expected under the null hypothesis. An introduction to the basic principles of hypothesis testing can be found in Cowan (1998, Chapter 4). Different goodness-of-fit tests are extensively described in, e.g, D'Agostino and Stephens (1986) and Gibbons and Chakraborti (2010, Chapter 4).

\subsubsection{Kolmogorov-Smirnov test}

Consider a sample of $N$ observations $x_{1}, \ldots, x_{N}$ obtained from a series of repeated measurements of a certain quantity. Our goal is to test whether these measurements are realizations from $N$ independent random variables distributed according to the same distribution function (null hypothesis $H_{0}$ ). The Kolmogorov-Smirnov statistic is based on the difference between the cumulative distribution expected under the null hypothesis $F_{X}(x)$ and the observed cumulative probabilities.

The cumulative distribution function of the sample, also called the empirical distribution function (EDF), is an estimate of the observed cumulative probabilities. The EDF 
$S_{N}(x)$ is defined as

$$
S_{N}(x)=\frac{\text { number of sample values } \leq x}{N} .
$$

If the sample observations are distinct and arranged in the increasing order $x_{(1)}<x_{(2)}<$ $\ldots<x_{(N)}$, a formal definition of $S_{N}(x)$ is

$$
S_{N}(x)= \begin{cases}0 & \text { if } x<x_{(1)} \\ i / N & \text { if } x_{(i-1)} \leq x<x_{(i)}, \quad i=1, \ldots, N \\ 1 & \text { if } x \geq x_{(N)}\end{cases}
$$

The EDF (3.88) thus represents a "stepwise" version of the cumulative distribution function, where the jumps occur at the ordered sample values and the jump heights equal the relative frequency of the observations. If an observation occurs $k$ times, the height of the respective jump is $k / N$.

If the observations $x_{1}, \ldots, x_{N}$ are a random sample drawn from the same cumulative distribution $F_{X}(x)$, the EDF $S_{N}(x)$ approaches $F_{X}(x)$ as $N \rightarrow \infty$ (see, e.g., Gibbons and Chakraborti 2010, Chapter 2). This result suggests that the statistic

$$
D_{N}=\sup _{x}\left|S_{N}(x)-F_{X}(x)\right|
$$

is a reasonable measure for the accuracy of the null hypothesis. If the null hypothesis is true, the data can be certainly represented as random variables with CDFs $F_{X}(x)$ and therefore $D_{N}$ is small. The quantity (3.89) is known as the Kolmogorov-Smirnov (KS) statistic.

The most important property of the KS statistic $D_{N}$ is that its probability distribution does not explicitly depend on the null hypothesis (distribution-free statistic). The distribution of $D_{N}$ must therefore be a function of the data only. For what difficult seems to believe, this property descends from the fact that any CDF can be mapped into a standard uniform distribution (see Section 3.4.1.1). A rigorous proof of this result can be found in, e.g., Gibbons and Chakraborti (2010, Theorem 3.1). Moreover, a convenient approximation to the distribution function $F\left(D_{N} \leq d\right)$ ) exists for large samples (Gibbons and Chakraborti 2010, Theorem 3.3).

The use of the KS statistics in a goodness-of-fit problem proceeds as follows. Consider a sample of $N$ observations $x_{1}, \ldots, x_{N}$ and the null hypothesis

$$
H_{0}: \quad F_{X}(x \mid \boldsymbol{\theta})=F_{0}(x \mid \boldsymbol{\theta}),
$$

where $F_{0}(x)$ is continuous $\mathrm{CDF}$ with known (specified or alternatively estimated) parameters $\boldsymbol{\theta}$. Firstly, the KS statistic $D_{N}$ is calculated from the sample and under $H_{0}$ obtaining a certain value $d^{*}$. Since the PDF $f\left(D_{N}\right)$ is known, we can calculate the probability that the KS statistic is larger than the observed value $d^{*}$

$$
\alpha_{K S}=\mathrm{P}\left(D_{N}>d^{*}\right)=\int_{d^{*}}^{\infty} f\left(D_{N}\right) d D_{N} .
$$

The right tail probability of the test statistic given above is generally referred to as the p-value. The null hypothesis $H_{0}$ is rejected if the p-value is smaller than a small, prescribed threshold value $\alpha$ known as the significance level. Contrary to the p-value, the 
significance level does not depend on the underlying hypothesis and is defined before conducting the experiment and acquiring the data. Typical values for the significance level $\alpha$ are $5 \%$ or $1 \%$. A p-value smaller than the suggested significance level indicates that the observed result would be highly unlikely under the null hypothesis. The smaller the $\mathrm{p}$-value is, the larger the significance of the rejection.

\subsubsection{Anderson-Darling test}

In this section we briefly describe the Anderson-Darling (AD) goodness-of-fit test. The AD statistic $A_{N}$ is a quadratic measure of the difference between the cumulative distribution $F_{X}(x)$ and the empirical distribution function $S_{N}(x)$. The AD statistic is defined by

$$
A_{N}=N \int_{-\infty}^{\infty} w(x)\left[S_{N}(x)-F_{X}(x)\right]^{2} d F_{X}(x),
$$

where $w(x)$ is the weighting function

$$
w(x)=\frac{1}{F_{X}(x)\left[1-F_{X}(x)\right]} .
$$

Since the weighting factor $w(x)$ emphasizes the observations in the tail of the distribution, this statistic is particularly useful for testing the capability of a given model in describing extreme data values.

The use of the AD statistic in a goodness-of-fit problem follows the same reasoning of the KS statistic. This has already been described in the previous section and it is therefore not repeated here. In the following, we refer to the AD p-value obtained for a given data sample and under a null hypothesis $H_{0}$ to as $\alpha_{A D}$.

\subsection{The Bayesian approach}

In the following we discuss the Bayesian approach to the problems of parameter estimation and model selection. In Section 3.7.1 we derive a general expression for the posterior distribution of the free parameters of a given model. The question of the prior choice is addressed in Section 3.7.2. Sections 3.7.3 and 3.7.4 finally concern with the problem of model selection among a set of alternatives. Introductory textbooks on Bayesian probability theory with particular attention to data analysis problems are, e.g., Sivia (1996) and Gregory (2005). An introduction to Bayesian reasoning and its applications can also be found in D’Agostini (2003).

\subsubsection{Parameter estimation}

In this section we describe the basics of Bayesian inference. The discussion is based on the fundamental concepts introduced in Section 3.1 and on Bayes' theorem (3.4).

Consider a model $M$ believed to describe a certain physical phenomenon and let $\boldsymbol{\theta}=\left(\theta_{1}, \ldots, \theta_{K}\right)$ be the $K$-dimensional vector of the model free parameters. To validate the model $M$, an experiment is conducted and a collection of $N$ measurements $\mathbf{x}=\left(x_{1}, \ldots, x_{N}\right)$ is recorded. Our aim is to characterize the agreement of $M$ with the 
data to decide whether accepting or rejecting the model itself. As discussed in the previous sections, a frequentist approach to the problem would test the reliability of the hypothesized model based on the estimates $\hat{\boldsymbol{\theta}}$ of its parameters which best fit the data. On the contrary, Bayesian inference permits to determine the probability distribution of the model parameters $\boldsymbol{\theta}$ themselves after observing the data. This gives information about the accuracy of the model in its own domain of definition and not just based on the estimated parameter values.

We now consider the model $M$ to be a certain probability density $f_{X}(\mathbf{x} \mid \boldsymbol{\theta})$. Bayes' theorem (3.4) allows to write the posterior probability of the model parameters $\mathrm{P}(\boldsymbol{\theta} \mid \mathbf{x}, M)$ as

$$
\mathrm{P}(\boldsymbol{\theta} \mid \mathbf{x}, M)=\frac{\mathrm{P}(\mathbf{x} \mid \boldsymbol{\theta}, M) \pi(\boldsymbol{\theta} \mid M)}{\mathrm{P}(\mathbf{x} \mid M)} .
$$

As already discussed in Section 3.1.1.2, the likelihood of the data sample $\mathrm{P}(\mathbf{x} \mid \boldsymbol{\theta}, M)$ represents the probability of observing the data $\mathbf{x}$ having assumed $M$ to be the true model with parameters $\boldsymbol{\theta}$. The prior probability $\pi(\boldsymbol{\theta} \mid M)$ describes the degree of belief in the model parameter values before conducting the experiment (and therefore before recording any data $\mathbf{x})$. By means of the continuous version of the law of total probability (3.5), the evidence $\mathrm{P}(\mathbf{x} \mid M)$ in $(3.93)$ is

$$
\mathrm{P}(\mathbf{x} \mid M)=\int \mathrm{P}(\mathbf{x} \mid \boldsymbol{\theta}, M) \pi(\boldsymbol{\theta} \mid M) d \boldsymbol{\theta} .
$$

Substituting (3.94) into (3.93) yields

$$
\mathrm{P}(\boldsymbol{\theta} \mid \mathbf{x}, M)=\frac{\mathrm{P}(\mathbf{x} \mid \boldsymbol{\theta}, M) \pi(\boldsymbol{\theta} \mid M)}{\int \mathrm{P}(\mathbf{x} \mid \boldsymbol{\theta}, M) \pi(\boldsymbol{\theta} \mid M) d \boldsymbol{\theta}} .
$$

The expression above is the joint distribution density of the model parameters $\boldsymbol{\theta}$ and is the most general formula of inductive Bayesian inference. It provides the complete description of our state of knowledge on the model parameters. It is interesting to note that $M$ is included as part of all the conditional statements in (3.95). The model $M$ indeed represents the knowledge we have on the problem beforehand and any probability relation has therefore to be valid for the selected model. According to Equation (3.95), the posterior distribution is a PDF in the parameter space $\boldsymbol{\theta}$, while the likelihood $\mathrm{P}(\mathbf{x} \mid \boldsymbol{\theta}, M)$ is not a probability density. As will be discussed in Section 3.7.3, the evidence $\mathrm{P}(\mathbf{x} \mid M)$ is a crucial quantity when comparing different models while has the role of a trivial normalization factor in the context of parameter inference.

The posterior distribution (3.95) is usually described using rappresentative quantities such as its mean value or its mode. In particular, the mode of (3.95) is referred to as the maximum a posteriori (MAP). The spread of the posterior distribution is generally described by its standard deviation. Since the posterior density (3.95) gives the most complete statistical answer to the problem of parameter inference, it is generally a good practice to explicitly show it. In the case of complex posterior densities (e.g. multi-modal and/or highly skewed) the descriptive quantities listed above might indeed be biased.

Assuming the measurements $\mathbf{x}$ to be independent and identically distributed, the likelihood $\mathrm{P}(\mathbf{x} \mid \boldsymbol{\theta}, M)$ is

$$
\mathrm{P}(\mathbf{x} \mid \boldsymbol{\theta}, M)=\prod_{i=1}^{N} f_{X}\left(x_{i} \mid \boldsymbol{\theta}\right) .
$$


For consistency in the notation (see Section 3.5), the likelihood above will be denoted as $\mathcal{L}(\boldsymbol{\theta})$ and Equation (3.95) then reads

$$
\mathrm{P}(\boldsymbol{\theta} \mid \mathbf{x}, M)=\frac{\mathcal{L}(\boldsymbol{\theta}) \pi(\boldsymbol{\theta} \mid M)}{\int \mathcal{L}(\boldsymbol{\theta}) \pi(\boldsymbol{\theta} \mid M) d \boldsymbol{\theta}} .
$$

\subsubsection{Choice of priors}

In order to calculate the posterior density (3.95) (or (3.97) if the measurements are independent and identically distributed), we have to face the problem of the choice of the prior distribution $\pi(\boldsymbol{\theta} \mid M)$. As already mentioned in Section 3.1.1.2, there is no general and objective method for calculating such probabilities. As its name manifestly suggests, the prior indeed depends on our knowledge of the model parameters before observing the data. Priors are claimed to be the major weakness of Bayesian inference and are often subject to criticism. However, there are situations in which a prior knowledge effectively exists and one can take advantage of it. For example, if measurements of a certain quantity of interest have been previously performed, the posterior density obtained for the old experiment can play the role of the prior distribution in the new inference problem. Moreover, the prior choice naturally forces the basic assumptions to be clearly stated. This is not always the case in other frameworks of analysis where the underlying assumptions may be easily hidden.

In parameter inference it is often the case that upper and lower limits for the quantity of interest (rather than its exact distribution) are known a priori. In such case, a reasonable choice of the prior is to consider $\pi(\theta \mid M)$ as a uniform distribution for $\boldsymbol{\theta}_{\min } \leq \boldsymbol{\theta} \leq \boldsymbol{\theta}_{\max }$. The fact that any parameter value within a given range is equally probable a priori reflects a "flat" knowledge of the parameters beforehand.

We now discuss the importance of a reasonable prior choice and the effect of different choices on the posterior distribution with the following example. Consider the model $M$ to be the exponential PDF (3.28). Assume a uniform prior for its rate parameter $\lambda$ within $\left[\lambda_{\max }, \lambda_{\min }\right]$. The prior distribution then reads

$$
\pi(\lambda \mid \operatorname{Exp})=\frac{1}{\lambda_{\max }-\lambda_{\min }} \doteq \frac{1}{\Delta \lambda}
$$

for $\lambda_{\min } \leq \lambda \leq \lambda_{\max }$ and is 0 otherwise. Bayes' theorem as given by (3.97) yields to the posterior distribution of the rate parameter

$$
\begin{aligned}
\mathrm{P}(\lambda \mid \mathbf{x}, \operatorname{Exp}) & =\frac{\mathcal{L}(\lambda) \pi(\lambda \mid \operatorname{Exp})}{\int_{\lambda_{\min }}^{\lambda_{\max }} \mathcal{L}(\lambda) \pi(\lambda \mid \operatorname{Exp}) d \lambda} \\
& =\frac{\lambda^{N} \exp \left[-\lambda \sum_{i=1}^{N} x_{i}\right]}{\int_{\lambda_{\min }}^{\lambda_{\max }} \lambda^{N} \exp \left[-\lambda \sum_{i=1}^{N} x_{i}\right] d \lambda} .
\end{aligned}
$$

For a uniform prior, the posterior density thus always coincides with the normalized likelihood of the data. For $N=0$ (no data recorded yet) the posterior density (3.99) simply reduces to the prior PDF in (3.98). Before performing any experiment, our knowledge about the rate parameter indeed coincides with the prior information as expected. 
We now perform a numerical experiment where the data $x_{i}$ in (3.99) are drawn from an exponential distribution with rate $\lambda=1$ and we also assume $\lambda_{\min }=0$ and $\lambda_{\max }=5$. Figure 3.14 illustrates the evolution of the posterior density (3.99) increasing the number of observations $N$. For the lowest number of observations $(N=5)$, the posterior density has the largest variance and is positively skewed. Increasing $N$ progressively decreases the variance of the posterior distribution and its MAP approaches the true value $\lambda=1$. For $N=50$ the posterior density is practically indistinguishable from a normal distribution as expected in the asymptotic limit of the likelihood function (Section 3.5.3). If a model is well supported by the data, as it is the case in this example, the posterior distribution peaks around the true value of the model parameter(s). The prior only mildly influence the posterior distribution in such cases and therefore the likelihood function dominates. If the prior does not include the true model parameters values, however, the posterior distribution results totally biased by the wrong prior assumption.

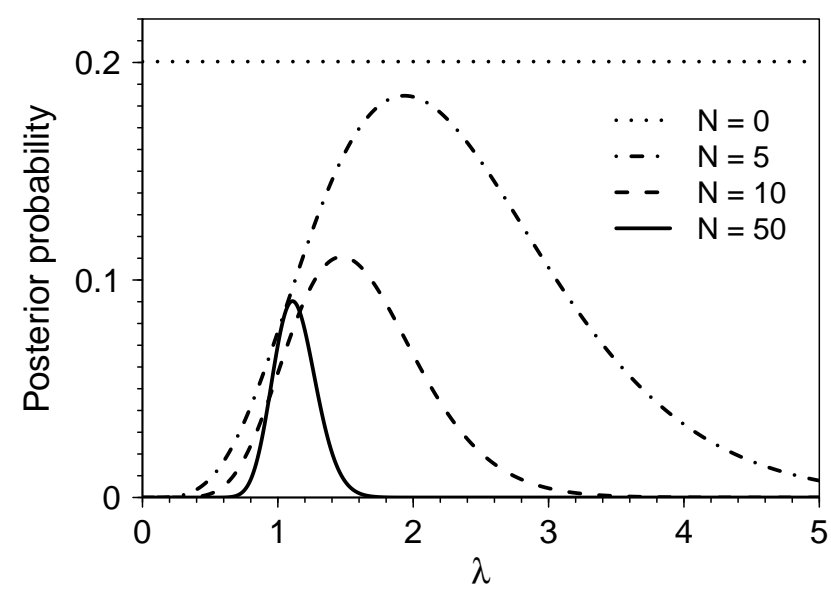

Figure 3.14: Posterior density (3.99) for a different number $N$ of random deviates drawn from an exponential distribution with rate $\lambda=1$. The prior is assumed to be uniform in $[0,5]$. The dotted line refers to $N=0$ where the posterior distribution coincides with the prior (the other distributions have been rescaled for plotting purposes).

\subsubsection{Model selection}

In the previous sections we described the Bayesian approach to the problem of estimating the parameters of a prescribed model. If alternative models are available, the question of which model best describes certain observations naturally arises. In the following we discuss the Bayesian answer to this problem.

Given a set of alternative models, one can be easily tempted to privilege the model which best fits the experimental measurements. This reasoning shows its fallacy considering the fact that more complex models, i.e. models with a higher number of free parameters, always better agree with the observations. The problem of model selection therefore reduces in quantifying the gain of information achieved for a more complex model. As will become clear in the following, Bayes' theorem can be used to quantify such gain. 
Consider a set of $Q$ alternative models $\left\{M_{i}\right\}_{i=1}^{Q}$ defined by a number of adjustable parameters and the experimental observations $\mathbf{x}$. The problem of model selection consists in estimating the probability of each model given the measurements $\mathbf{x}$ :

$$
\mathrm{P}\left(M_{i} \mid \mathbf{x}\right), \quad i=1, \ldots, Q .
$$

The sample space constituted by the selected models must contain the true model by construction and therefore the probabilities above satisfy

$$
\sum_{i=1}^{Q} \mathrm{P}\left(M_{i} \mid \mathbf{x}\right)=1
$$

Using Bayes' theorem (3.4), the probability of the $i$-th model can be written as

$$
\begin{aligned}
\mathrm{P}\left(M_{i} \mid \mathbf{x}\right) & =\frac{\mathrm{P}\left(\mathbf{x} \mid M_{i}\right) \mathrm{P}\left(M_{i}\right)}{\mathrm{P}(\mathbf{x})} \\
& =\frac{\mathrm{P}\left(\mathbf{x} \mid M_{i}\right) \mathrm{P}\left(M_{i}\right)}{\sum_{i=1}^{Q} \mathrm{P}\left(\mathbf{x} \mid M_{i}\right) \mathrm{P}\left(M_{i}\right)},
\end{aligned}
$$

where we used the law of total probability (3.5) in the last equality. In the expression above, the probability $\mathrm{P}\left(M_{i}\right)$ must be assigned before the analysis of the data $\mathbf{x}$ and thus represents the prior belief in model $M_{i}$, while the likelihood $\mathrm{P}\left(\mathbf{x} \mid M_{i}\right)$ is the marginal probability of the data (3.94). Equation (3.102) then explicitly reads

$$
\mathrm{P}\left(M_{i} \mid \mathbf{x}\right)=\frac{\int \mathrm{P}\left(\mathbf{x} \mid \boldsymbol{\theta}_{i}, M_{i}\right) \pi\left(\boldsymbol{\theta}_{i} \mid M_{i}\right) d \boldsymbol{\theta}_{i} \mathrm{P}\left(M_{i}\right)}{\sum_{i=1}^{Q} \int \mathrm{P}\left(\mathbf{x} \mid \boldsymbol{\theta}_{i}, M_{i}\right) \pi\left(\boldsymbol{\theta}_{i} \mid M_{i}\right) d \boldsymbol{\theta}_{i} \mathrm{P}\left(M_{i}\right)},
$$

where $\boldsymbol{\theta}_{i}$ represents the vector of parameters of model $M_{i}$.

To determine the relative merit of the two models $M_{i}$ and $M_{j}$ among a set of alternatives, it is useful to consider the ratio of the model posterior probabilities

$$
O_{i j} \doteq \frac{\mathrm{P}\left(M_{i} \mid \mathbf{x}\right)}{\mathrm{P}\left(M_{j} \mid \mathbf{x}\right)}
$$

which is the so-called posterior odds ratio. Using (3.102) and (3.103), the posterior odds ratio reads

$$
\begin{aligned}
O_{i j} & =\frac{\mathrm{P}\left(\mathbf{x} \mid M_{i}\right)}{\mathrm{P}\left(\mathbf{x} \mid M_{j}\right)} \frac{\mathrm{P}\left(M_{i}\right)}{\mathrm{P}\left(M_{j}\right)} \\
& =\frac{\int \mathrm{P}\left(\mathbf{x} \mid \boldsymbol{\theta}_{i}, M_{i}\right) \pi\left(\boldsymbol{\theta}_{i} \mid M_{i}\right) d \boldsymbol{\theta}_{i}}{\int \mathrm{P}\left(\mathbf{x} \mid \boldsymbol{\theta}_{j}, M_{j}\right) \pi\left(\boldsymbol{\theta}_{j} \mid M_{j}\right) d \boldsymbol{\theta}_{j}} \frac{\mathrm{P}\left(M_{i}\right)}{\mathrm{P}\left(M_{j}\right)} .
\end{aligned}
$$

The first factor on the RHS of (3.106) is the ratio of the integrated likelihoods of the two models and is called the Bayes' factor for $M_{i}$ against $M_{j}$ (usually denoted by $B_{i j}$ ). The second factor on the RHS of (3.106) is known as the prior odds ratio. If there is no a priori preference for any of the models, the prior odds ratio can be set to 1 . The posterior odds $O_{i j}$ coincides then with the Bayes' factor. Due to (3.101), this is formally equivalent to assign a prior probability $1 / Q$ to each of the models. If $B_{i j} \gg 1$ for any $j \neq i$, a strong evidence in favor of $M_{i}$ exists. On the contrary, $M_{j}$ is preferred if $B_{i j} \ll 1$. If $B_{i j} \approx 1$, an informed judgement based on the current data is not possible. Jeffreys (1961) proposed some rules of thumb for interpreting $B_{i j}$. Though only the posterior probability $\mathrm{P}\left(M_{i} \mid \mathbf{x}\right)$ exactly quantifies how likely is a certain model in the light of the data, Jeffreys' rules are generally adopted standards of evidence in scientific investigation. 


\subsubsection{A built-in Occam's razor}

In the previous section we derived an expression for the comparison of hypotheses in a Bayesian framework. The simplest case in which two alternative models are available, namely $M_{1}$ and $M_{2}$ characterized by $K_{1}$ and $K_{2}$ free parameters respectively, reveals interesting insights. Assuming the two models to be equally likely a priori, the prior model probabilities are $\mathrm{P}\left(M_{1}\right)=\mathrm{P}\left(M_{2}\right)=1 / 2$ and the posterior odds ratio (3.106) reads

$$
O_{12}=\frac{\int \mathrm{P}\left(\mathbf{x} \mid \boldsymbol{\theta}_{1}, M_{1}\right) \pi\left(\boldsymbol{\theta}_{1} \mid M_{1}\right) d \boldsymbol{\theta}_{1}}{\int \mathrm{P}\left(\mathbf{x} \mid \boldsymbol{\theta}_{2}, M_{2}\right) \pi\left(\boldsymbol{\theta}_{2} \mid M_{2}\right) d \boldsymbol{\theta}_{2}} .
$$

Furthermore, assume the priors of the models parameters $\pi\left(\boldsymbol{\theta}_{1} \mid M_{1}\right)$ and $\pi\left(\boldsymbol{\theta}_{2} \mid M_{2}\right)$ to be uniform within the intervals $\left[\theta_{1, \min }^{k}, \theta_{1, \text { max }}^{k}\right]$, for $k=1, \ldots, K_{1}$, and $\left[\theta_{2, \min }^{k}, \theta_{2, \text { max }}^{k}\right.$, for $k=$ $1, \ldots, K_{2}$, respectively. Equation (3.107) then yields

$$
O_{12}=\frac{\prod_{k=1}^{K_{2}} \Delta \theta_{2}^{k}}{\prod_{k=1}^{K_{1}} \Delta \theta_{1}^{k}} \frac{\int \mathrm{P}\left(\mathbf{x} \mid \boldsymbol{\theta}_{1}, M_{1}\right) d \boldsymbol{\theta}_{1}}{\int \mathrm{P}\left(\mathbf{x} \mid \boldsymbol{\theta}_{2}, M_{2}\right) d \boldsymbol{\theta}_{2}},
$$

where $\Delta \theta_{1}^{k} \doteq \theta_{1, \text { max }}^{k}-\theta_{1, \text { min }}^{k}$ and $\Delta \theta_{2}^{k} \doteq \theta_{2, \text { max }}^{k}-\theta_{2, \text { min }}^{k}$.

Let $M_{2}$ have a larger number of free parameters $\left(K_{2}>K_{1}\right)$ and let $\Delta \theta_{1}^{k}=\Delta \theta_{2}^{k}$ for $k=1, \ldots, K_{1}$. In this case the prior probabilities ratio in (3.108) is larger than one and thus acts in favoring $M_{1}$ with respect to $M_{2}$. Model $M_{2}$, having a larger number of degrees of freedom, results therefore systematically penalized with respect to the simpler one. This follows the common scientific practice where we usually resort to the Occam's razor in discarding unnecessarily complicated models. For example, it is well understood that a polynomial of order $n-1$, despite offering a perfect fit of $n$ experimental points, it is generally not regarded as the best description of the data. This reasoning naturally comes out in Bayesian model selection problems. For this reason the first factor in (3.108) is often called the Occam's factor.

The Occam's factor is, however, weighted by the ratio of integrated likelihoods which describes the relative quality of the models fit to the data. Therefore, the posterior odds ratio (3.108) represents a trade-off between the ability of each model in describing the data and the model complexity itself. As a final remark, consider the case where the two models fit the data roughly equally well, thus having a ratio of integrated likelihoods of approximately one. The posterior odds ratio results then dominated by the priors. Moreover, if the two models have the same number of free parameters $\left(K_{1}=K_{2}\right)$, our prior knowledge on these parameters may completely bias the result. The model having a narrower range of parameters values accessible a priori results thus favored over the less discriminant one. 



\section{Results from geomagnetic polarity time scales}

In this chapter we analyze the statistical properties of geomagnetic polarity epochs using the methods described in Chapter 3 . As previously discussed in Section 1.4.4 geomagnetic polarity time scales provide the approximate times at which the geomagnetic field reversed its polarity. These records are constantly revised and updated. The reversal chronologies selected for this study are described in Section 4.1 and their statistical analysis is presented in Section 4.2. Since resolution problems become important during the Mesozoic era and are overwhelming before, we restricted our analysis to reversals younger than 170 million years.

\subsection{Selection of geomagnetic polarity time scales}

Geomagnetic polarity time scales (GPTSs) are constructed dating oceanic magnetic anomalies and differ for the various crustal age interpolations used (Section 1.4.4). Due to the poor data resolution, the polarity changes are regarded as instantaneous. Table 4.1 lists the GPTSs selected for the subsequent statistical analysis together with some preliminary statistical information.

Younger and most reliable chronologies cover the period of the Late Cretaceous through the Neogene (C-sequence), thus roughly spanning the last $83 \mathrm{Myr}$ after the Cretaceous normal superchron (CNS). Cande and Kent (1995) claimed to produce a record with uniform resolution throughout and containing almost all polarity intervals longer than $30 \mathrm{kyr}$. Hereafter, we refer to this time scale to as CK95 and its polarity sequence is shown in the upper panel of Figure 4.1.

The reversal frequency seems to increase after the CNS and potential variations in the underlying reversal rate (see also Figure 1.9) have been already discussed in Section 1.4.5. Distributions with time-dependent parameters have been proposed in order to capture the long-term reversal rate variations and ultimately the superchron (McFadden and Merrill 1984. Constable 2000). Such models assume that external effects, most often of mantle origin, influence the core field dynamics. However, it is not entirely clear if external mechanisms are necessarily required to capture the observed variations. Several studies demonstrated that long-term stationary processes are also able to reproduce the statistics of geomagnetic reversal occurrences (see Hulot and Gallet 2003, Ryan and Sarson 2007, Jonkers 2007, Shcherbakov and Fabian 2012, and the discussion in Section 1.4.5). Following these latter studies, our statistical analysis assumes a stationary reversal rate throughout the GPTSs. A suite of distribution functions, defined by time independent 


\begin{tabular}{|c|c|c|c|c|c|c|}
\hline & Name & Reference & Chron & $\begin{array}{c}\text { Age range } \\
{[\mathrm{Ma}]}\end{array}$ & $N$ & $\begin{array}{c}\overline{\Delta t} \\
{[\mathrm{Myr}]}\end{array}$ \\
\hline \multirow{6}{*}{ 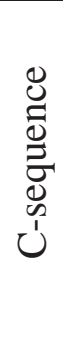 } & CK95 & Cande and Kent (1995) & $\mathrm{C} 1 \mathrm{n}-\mathrm{C} 34 \mathrm{n}$ & $0-118$ & 184 & 0.637 \\
\hline & CK95cc & Cande and Kent (1995) & $\mathrm{C} 1 \mathrm{n}-\mathrm{C} 34 \mathrm{n}$ & $0-118$ & 292 & 0.402 \\
\hline & CK95-CNS & Cande and Kent (1995) & $\mathrm{C} 1 \mathrm{n}-\mathrm{C} 33 \mathrm{r}$ & $0-83$ & 183 & 0.449 \\
\hline & CK95cc-CNS & Cande and Kent (1995) & $\mathrm{C} 1 \mathrm{n}-\mathrm{C} 33 \mathrm{r}$ & $0-83$ & 291 & 0.284 \\
\hline & $\mathrm{O} 12 \mathrm{C}$ & Ogg (2012) & $\mathrm{C} 1 \mathrm{n}-\mathrm{C} 34 \mathrm{n}$ & $0-126$ & 190 & 0.659 \\
\hline & O12C-CNS & Ogg (2012) & $\mathrm{C} 1 \mathrm{n}-\mathrm{C} 34 \mathrm{n}$ & $0-126$ & 189 & 0.478 \\
\hline \multirow{5}{*}{$\begin{array}{l}\dot{\mathscr{d}} \\
\sum^{1} \\
\sum^{1}\end{array}$} & TS10 & Tominaga and Sager (2010) & M0r-M29r & $124-158$ & 100 & 0.337 \\
\hline & MHTC12 & Malinverno et al. (2012) & M0r-M30n & $121-156$ & 101 & 0.345 \\
\hline & $\mathrm{O} 12 \mathrm{M}$ & Ogg (2012) & M0r-M45n & $126-171$ & 258 & 0.173 \\
\hline & $\mathrm{O} 12$ & Ogg (2012) & $\mathrm{C} 1 \mathrm{n}-\mathrm{M} 45 \mathrm{n}$ & $0-171$ & 448 & 0.379 \\
\hline & O12-CNS & Ogg (2012) & C1n-M45n & $0-171$ & 447 & 0.302 \\
\hline
\end{tabular}

Table 4.1: Geomagnetic polarity time scales used in this study and relative references. Covered chrons, age range, number of chrons $N$ and mean chron duration $\overline{\Delta t}$ are listed in column 3 to 6 respectively. See the main text for further details. 
parameters and described in Section 3.4.1 will be fitted to the observed chron durations. Using a Bayesian approach, we therefore seek for the model which best describes the data.
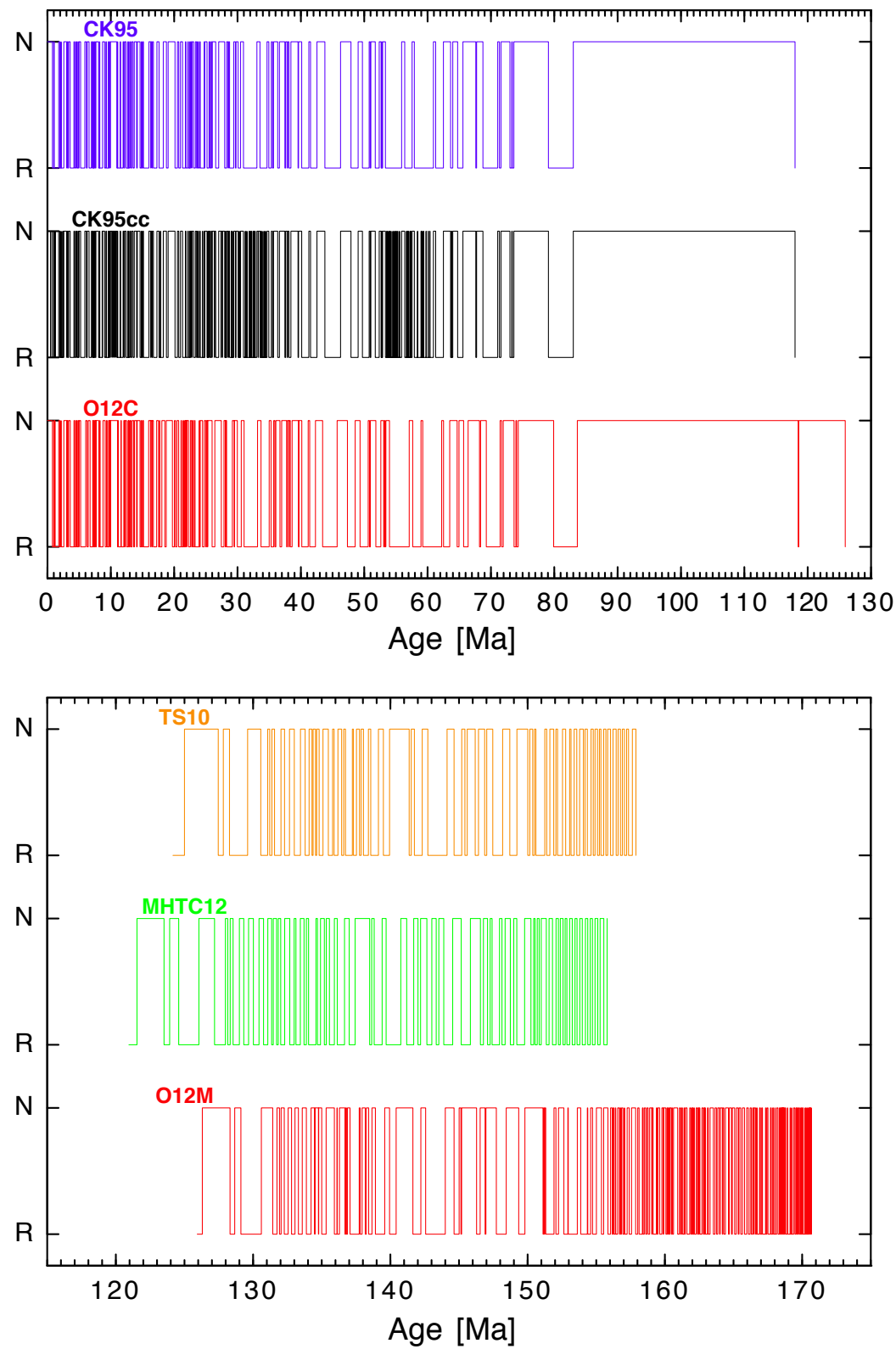

Figure 4.1: Polarity sequences for the Cenozoic era (top panel) and the Mesozoic era (bottom panel). Normal and reverse polarities are indicated by the letter $\mathrm{N}$ and $\mathrm{R}$ respectively. Each sequence is labeled with the name assigned to the respective geomagnetic polarity time scale (see Table 4.1). Cenozoic sequences start from chron C1n and Mesozoic ones from chron M0r.

Cande and Kent (1995) listed 108 brief polarity intervals which are less constrained by the magnetic anomaly record. These events, typically shorter than $30 \mathrm{kyr}$, are known as cryptochrons. Hereafter, we refer to the GPTS including cryptochrons to as CK95cc and its polarity sequence is shown in the upper panel of Figure 4.1 (black sequence). This 
version of the GPTS permits to study the impact of additional brief events on the chron statistics. However, it is likely that several cryptochrons reflect strong paleointensity field variations rather than true polarity changes.

Cenozoic GPTSs where we artificially discarded the CNS are distinguished from the original versions by means of the suffix "-CNS" in the respective names. We will test the compatibility of the CNS with a selected statistical model making use of these modified GPTSs. The duration of the CNS is about $35 \mathrm{Myr}$, more than 50 times longer than the average chron duration and about 6 times longer than the second longest chron. As already discussed in Section 1.4.5, the question whether the CNS is an outlier or an extreme event compatible with the large variety of time scales produced by the geodynamo is still a matter of debate. Discarding the superchron from the actual set of events would therefore serve for testing its compatibility with a given statistical model.

Since times of reversal occurrences during the Middle Jurassic through the Early Cretaceous (M-sequence, $121-158 \mathrm{Ma}$ ) are affected by larger uncertainties, we used the two independent GPTSs of Tominaga and Sager (2010) (TS10) and Malinverno et al. (2012) (MHTC12) for this older period. The latter chronology includes one event more than the former, while their mean chron durations are comparable with a value of about $0.34 \mathrm{Myr}$ (Table 4.1). Clearly different reversal timings, mainly caused by the different crustal age interpolations used, are evident from the polarity sequences in Figure 4.1 (bottom panel).

Finally, the last GPTS selected for our analysis is the chronology of Ogg (2012) which comprises the $\mathrm{C}$-sequence and an extended version of the M-sequence. We refer to this reversal chronology to as $\mathrm{O} 12$ hereafter. $\mathrm{O} 12$ includes 448 chrons spanning the period from $171 \mathrm{Ma}$ to the present. The extension of the M-sequence comprises chrons M27r-M45n and cover the oldest $14 \mathrm{Myr}$. Contrary to younger periods, the oldest magnetic anomalies have been recorded using magnetometers towed near the oceanic crust. This inevitably introduces short-wavelength features in the record which might not reflect true polarity changes of the field. Reversal rates as high as $10 \mathrm{Myr}^{-1}$ are observed during the oldest $14 \mathrm{Myr}$ of $\mathrm{O} 12$ (Figures 1.9 and 4.1) and are likely an artifact of the different recording method. Further details regarding this GPTS can be found in Section 1.4.4. Despite these problems, we will use the extended chronology $\mathrm{O} 12$ in order to study the potential impact of briefer events on the statistic. Restricted versions of O12 for the Cenozoic and Mesozoic periods, named $\mathrm{O} 12 \mathrm{C}$ and $\mathrm{O} 12 \mathrm{M}$ respectively, have been considered for comparison with the other GPTSs.

Figure 4.2 shows the distribution of chron durations for each of the selected GPTSs obtained using an adaptive bin size which guarantees a smoother probability density without empty bins. The maximum chron duration has been limited to $1.3 \mathrm{Myr}$ for plotting purposes only. We estimated this probability density as follows. Consider the set of chron durations $\left\{\Delta t_{i}\right\}_{i=1}^{N}$ to be sorted in ascending order and a number $M<N$ of such events. The variable bins have been centered at $\Delta t_{n}$ and have boundaries $\left(\Delta t_{n}+\Delta t_{n-M}\right) / 2$ and $\left(\Delta t_{n}+\Delta t_{n+M}\right) / 2$. The estimated probability density in the $n$-th bin is therefore

$$
p_{n}=\frac{c_{n}}{N} \frac{2}{\Delta t_{n+M}-\Delta t_{n-M}},
$$

where $c_{n}$ is the number of events in the bin. The variance $\sigma^{2}$ of the probability density 

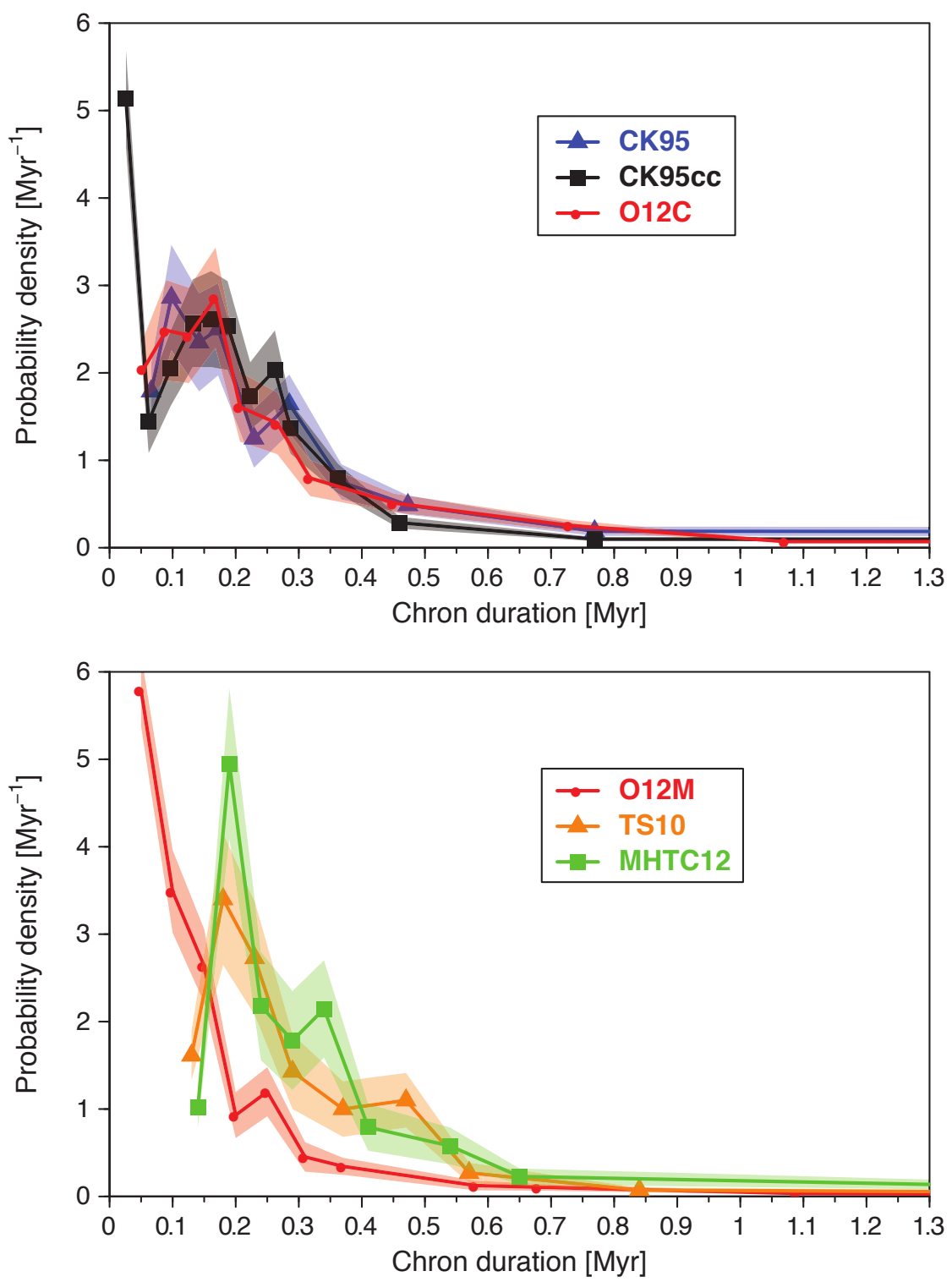

Figure 4.2: Distributions of chron durations for the Cenozoic (top panel) and the Mesozoic (bottom panel) as recorded by the different geomagnetic polarity time scales selected (see legend insets and refer to Table 4.1). The horizontal axis has been limited to $1.3 \mathrm{Myr}$ for plotting purposes. Color shaded areas mark the $95 \%$ confidence intervals around the estimated probability density (see the main text for more details).

(4.1) can be estimated assuming that the counts $c_{n}$ follow a multinomial distribution:

$$
\sigma^{2}\left[p_{n}\right]=\left[\frac{2}{N\left(\Delta t_{n+M}-\Delta t_{n-M}\right)}\right]^{2} c_{n}\left(1-\frac{c_{n}}{N}\right) .
$$

Color shaded areas in Figure 4.2 correspond to the $2 \sigma$ intervals around the estimated probability densities.

CK95 and O12C have similar distributions but the lower end of the former GPTS is significantly different if cryptochrons are included (Figure 4.2, top panel). These brief and less reliable events indeed constitute about $37 \%$ of the total number of chrons recorded 
for the past $118 \mathrm{Myr}$. Shorter polarity intervals last not less than $10 \mathrm{kyr}$. The CNS (not shown in Figure 4.2) has a duration of about $35 \mathrm{Myr}$, while the second longest chron has the considerably smaller duration of 5.6 Myr.

The distributions of chron durations for the M-sequence as reported by TS10 and MHTC12 do not seem to differ significantly despite the dating of certain events is greatly diverse (Figure 4.1, bottom panel). Polarity intervals of about $200 \mathrm{kyr}$ are the most likely and the two time scales closely agree on this estimate. On the contrary, O12M favors durations shorter than $100 \mathrm{kyr}$. As already discussed above, most of these relatively brief events are almost certainly an artifact of the different recording method employed in the extension of the oldest part of this GPTS. The longest chron recorded during the Mesozoic occurs slightly before the CNS and its duration is somewhat different between the GPTSs analyzed: TS10 and MHTC12 report values of 2.46 and $1.97 \mathrm{Myr}$ respectively, while dating from $\mathrm{O} 12 \mathrm{M}$ gives $2.02 \mathrm{Myr}$.

\subsection{Distribution of paleomagnetic polarity intervals}

We fitted the paleomagnetic polarity intervals from the GPTSs described in the previous section to a suite of distribution functions (exponential, gamma, Weibull, log-normal and log-logistic described in Section 3.4.1). Distribution parameters are estimated using the maximum likelihood method (Section 3.5) and the goodness-of-fit is evaluated by means of the Kolmogorov-Smirnov (KS) and Anderson-Darling (AD) statistics (Section 3.6). We quantified the relative likelihood of each model by means of the Bayesian posterior odds (3.106). Since no preference to any particular distribution is given a priori, we assumed equal prior model probabilities. Moreover, we considered uniform prior probabilities for the distribution parameters.

Figure 4.3 summarizes the results obtained from the frequentist and Bayesian approaches. The upper row depicts the AD p-values of the distribution fits of chron durations from each of the selected GPTSs. Only distribution fits with KS and AD p-values higher than $5 \%$ are plotted. Log-normal and log-logistic distributions describe the polarity interval durations from most of the selected records. The only exceptions occur for the GPTSs including cryptochrons (CK95cc and CK95cc-CNS) where all the selected models can be rejected at the $95 \%$ confidence level. Table 4.2 lists the estimated distribution parameters and their standard errors (3.82) for the two fitting models, together with the respective KS and $\mathrm{AD}$ p-values and posterior model probabilities.

Posterior probabilities of the distribution functions reveal further interesting insights (Figure 4.3, bottom row). The relative sizes of the symbols in the bottom row of Figure 4.3 correspond to the respective posterior odds ratios as given by Equations (3.104) and (3.106). Consider, in the first place, the Cenozoic sequence of chrons represented by CK95 and O12C. The evidence in favor of the log-logistic distribution is positive for both reversal chronologies (with a posterior model probability of $76 \%$ and $72 \%$ respectively), while the log-normal is the only second contender.

If the CNS is artificially discarded from each of the two records (CK95-CNS and O12C-CNS), the result reverses: the evidences in favor of the log-normal distribution are strong and its posterior probabilities reach $90 \%$ in both cases. The reason of such behavior resides in the tails of the two distributions. Log-normal and log-logistic are both heavy- 
tailed distribution functions, i.e. they decay at infinity slower than an exponential, but they belong to different classes. Let $\Delta t$ be the positive random variable describing chron durations. As $\Delta t \rightarrow \infty$, the log-logistic PDF (3.54) shows the pure power-law decay

$$
f(\Delta t \mid \alpha, \theta) \sim \Delta t^{-\alpha-1},
$$

while the log-normal decreases more quickly (Shcherbakov and Fabian 2012).

Figure 4.4 shows the best-fitting log-normal and log-logistic PDFs of chron durations from $\mathrm{O} 12 \mathrm{C}$. The data density is obtained using variable bin sizes as described in the previous section. The two models can be considered of comparable quality since they show a similar agreement with the data. The posterior odds ratio (3.106) of the log-logistic to the log-normal distribution is of 2.6 which indeed gives only a weak evidence in favor of the former model. The CNS (included in the last bin of Figure 4.4) is closer to the loglogistic rather than to the log-normal tail as expected. This implies that the maximum of the likelihood function (3.65) is larger for the log-logistic model. The maximum of the loglikelihood (3.67) is indeed -30.6 for the log-logistic, while it reaches the somewhat milder value of -32.6 for the log-normal. Discarding the CNS from O12C reverses the result: the log-logistic and log-normal distributions have log-likelihood maxima of -19.7 and -18.8 respectively. Despite being just a single event, the gain in likelihood introduced by the CNS in favor of the log-logistic distribution is thus sufficient to render this model the preferred alternative. However, the tail of the log-logistic distribution, decaying as $\Delta t^{-2.52}$ according to 4.3, is nevertheless underestimating the probability of the CNS occurrence by a significant margin (Figure 4.4). Jonkers (2003), fitting a pure power-law $\Delta t^{-\beta}$ to the

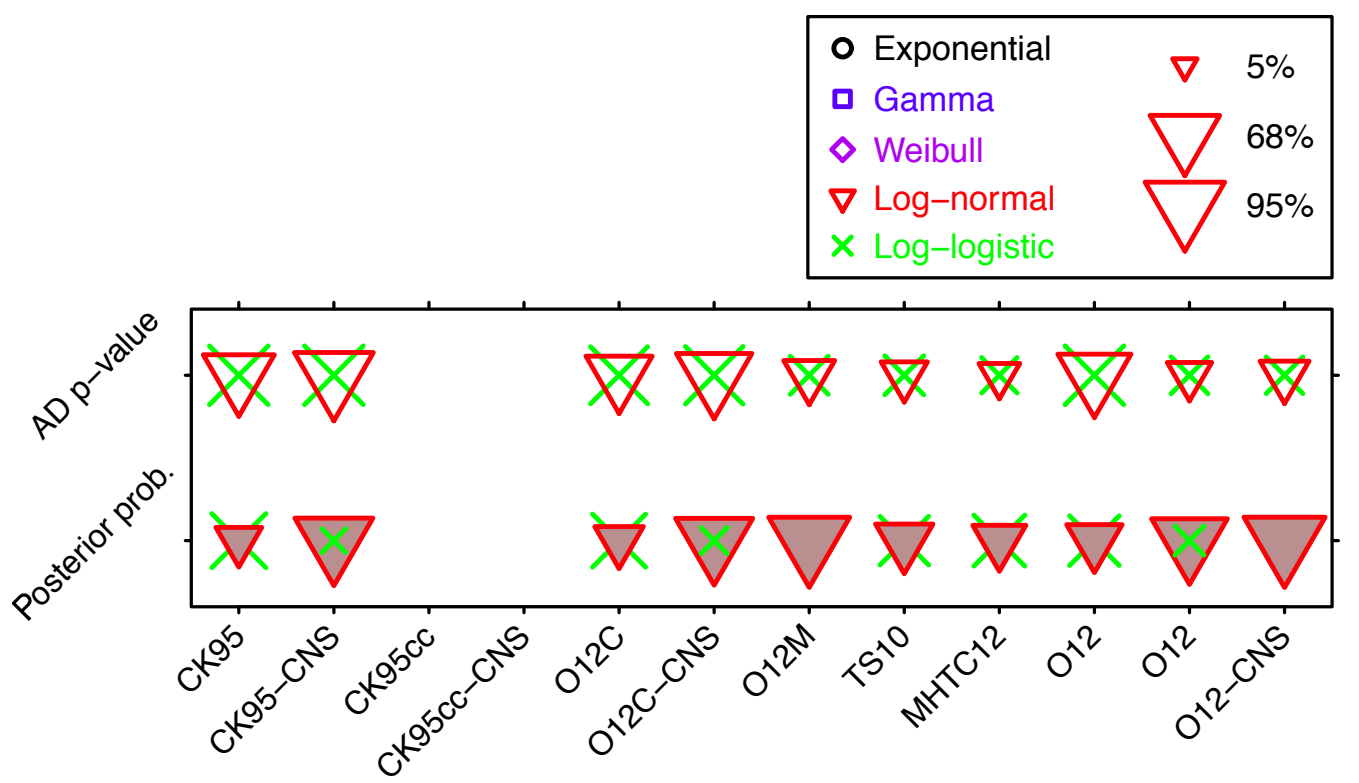

Figure 4.3: Anderson-Darling (AD) p-values (top row) and Bayesian posterior probabilities (3.103) (bottom row) of the tested distribution functions for chron durations from different geomagnetic polarity time scales (refer to Table 4.1 for the time scales acronyms). The tested distribution functions are marked with different colored symbols. Symbol sizes are scaled with the associated AD p-value (top row) or posterior probability (bottom row) as shown in the legend. 


\begin{tabular}{|c|c|c|c|c|c|c|c|c|c|c|c|c|}
\hline \multirow[t]{2}{*}{ Name } & \multicolumn{6}{|c|}{ Log-normal } & \multicolumn{6}{|c|}{ Log-logistic } \\
\hline & $\mu[\ln (\mathrm{Myr})]$ & $\sigma$ & $r$ & $\alpha_{K S}$ & $\alpha_{A D}$ & $\mathrm{P}$ & $\theta[\mathrm{Myr}]$ & $\alpha$ & $r$ & $\alpha_{K S}$ & $\alpha_{A D}$ & $\mathrm{P}$ \\
\hline CK95 & $-1.390 \pm 0.084$ & $139 \pm 0$. & 0.9 & 80.3 & 71.3 & 23.9 & $239 \pm 0.019$ & $583 \pm 0$ & -4.3 & 96.5 & 93.3 & 76.1 \\
\hline CK95-CNS & $-1.417 \pm 0.080$ & $1.081 \pm 0.057$ & 1.7 & 97.8 & 92.7 & 90.3 & $0.237 \pm 0.019$ & $1.625 \pm 0.100$ & -2.5 & 95.1 & 93.7 & 9.7 \\
\hline $\mathrm{O} 12 \mathrm{C}$ & $-1.417 \pm 0.086$ & $1.186 \pm 0.061$ & 0.08 & 22.3 & 59.8 & 27.5 & $0.231 \pm 0.019$ & $1.520 \pm 0.092$ & -4.7 & 74.8 & 87.1 & 72.5 \\
\hline O12C-CNS & $-1.444 \pm 0.082$ & $1.132 \pm 0.058$ & 0.4 & 36.5 & 83.0 & 87.8 & $0.228 \pm 0.019$ & $1.558 \pm 0.094$ & -7.9 & 81.8 & 89.1 & 12.2 \\
\hline TS10 & $-1.358 \pm 0.067$ & $0.674 \pm 0.048$ & -1.3 & 21.3 & 24.5 & 41.8 & $0.244 \pm 0.016$ & $2.639 \pm 0.219$ & -7.4 & 55.4 & 33.7 & $\overline{58.2}$ \\
\hline MHTC12 & $-1.273 \pm 0.059$ & $0.595 \pm 0.042$ & 0.6 & 17.0 & 17.9 & 34.5 & 0.267 & $2.992 \pm 0.247$ & -6.8 & 30.6 & 26.6 & 65.5 \\
\hline $\mathrm{O} 12 \mathrm{M}$ & $-2.327 \pm 0.064$ & $1.019 \pm 0.045$ & 1.5 & 27.3 & 31.2 & 99.2 & $0.094 \pm 0.006$ & $1.709 \pm 0.088$ & 0.9 & 33.6 & 31.5 & 0.8 \\
\hline $\mathrm{O} 12$ & & $82 \pm$ & -2.1 & 44.0 & 22.1 & 84.0 & 107 & $1.499 \pm$ & -3.7 & 28.8 & 29.4 & 16.0 \\
\hline O12-CNS & $-1.953 \pm 0.055$ & $1.154 \pm 0.039$ & 2.5 & 52.2 & 28.1 & 99.7 & $0.136 \pm 0.007$ & $1.516 \pm 0.059$ & -1.9 & 32.2 & 28.8 & 0.3 \\
\hline
\end{tabular}

Table 4.2: Statistical properties of the log-normal and log-logistic distributions describing chrons from the different geomagnetic polarity time scales (first column). Maximum likelihood estimates of distribution parameters are given with their standard errors in the second and third (eighth and ninth) columns for the log-normal (log-logistic) distribution. Remaining columns indicate: $r$ the Pearson correlation coefficient between the distribution parameters, $\alpha_{K S}$ and $\alpha_{A D}$ the Kolmogorov-Smirnov and Anderson-Darling p-values of the distribution fit, $\mathrm{P}$ the posterior probability of the distribution function. Correlation coefficients, $\mathrm{p}$-values and posterior probabilities are given in percentages. 


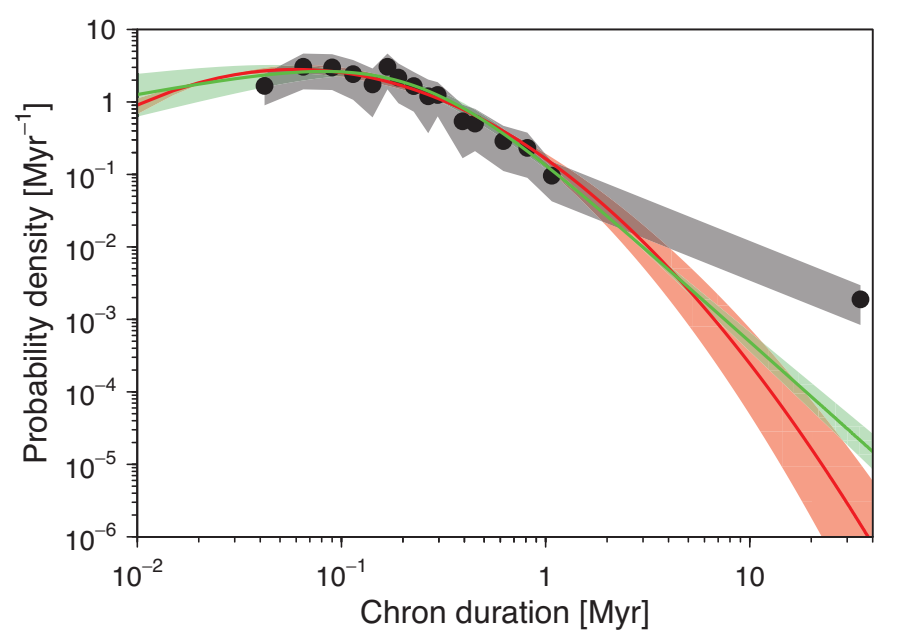

Figure 4.4: Probability density of geomagnetic chron durations for the Cenozoic time scale $\mathrm{O} 12 \mathrm{C}$ (cf. Table 4.1). The gray shaded region marks the $95 \%$ confidence interval for the binned probability density. Red and green curves show the best-fitting log-normal and log-logistic distributions respectively. Color shaded areas denote the $95 \%$ confidence intervals on the respective distribution parameters estimates.

binned chron durations from CK95, estimated a larger exponent of $\beta=1.09$ which better describes the data tail. However, a power-law distribution behaves questionably at lower chron durations where both the log-normal and the log-logistic closely agree with the data.

Consider now the M-sequence of chrons as recorded by TS10 and MHTC12. Though the reversals timings of these GPTSs significantly differ (Figure 4.1, bottom panel), the estimated parameters of the log-normal and log-logistic distributions agree in the limits of statistical errors (Table 4.2). The posterior odds ratios of the log-logistic to the lognormal distribution are 1.4 and 1.9 for TS10 and MHTC12 respectively and therefore an informed judgement on on the best model is impossible.

The estimated log-normal and log-logistic distribution parameters for the longer reversal chronology $\mathrm{O} 12 \mathrm{M}$ significantly differ from the respective estimates obtained for the Mesozoic time scales analyzed above. As already pointed out before, the reason of such differences can be attributed to the large fraction of short (not fully constrained) chrons recorded in the oldest $14 \mathrm{Myr}$ of O12M. Figure 4.5 presents a comparison of the distributions of chron durations from MHTC12 and O12M. Best-fitting log-normal and log-logistic PDFs are shown for each data set by the red and green curves respectively. Both distribution functions seem to fit the data relative to each GPTS equally well. The two models, however, seem to decay too slowly at low chron durations for the MHTC12 data. As already discussed above, no decisive evidence in favor of neither model exists for MHTC12. On the contrary, the posterior probability for the log-normal distribution is as high as $99 \%$ for O12M. Despite the statistical results are better constrained by the larger data sample of O12M, a strong bias might be introduced by the excess of short chrons recorded as an artifact of the measurement method employed for the oldest part of this GPTS. It is interesting to note that another outlier might be identified in the M-sequence. This is a chron lasting about $2 \mathrm{Myr}$ which neither the log-normal nor the log-logistic can 


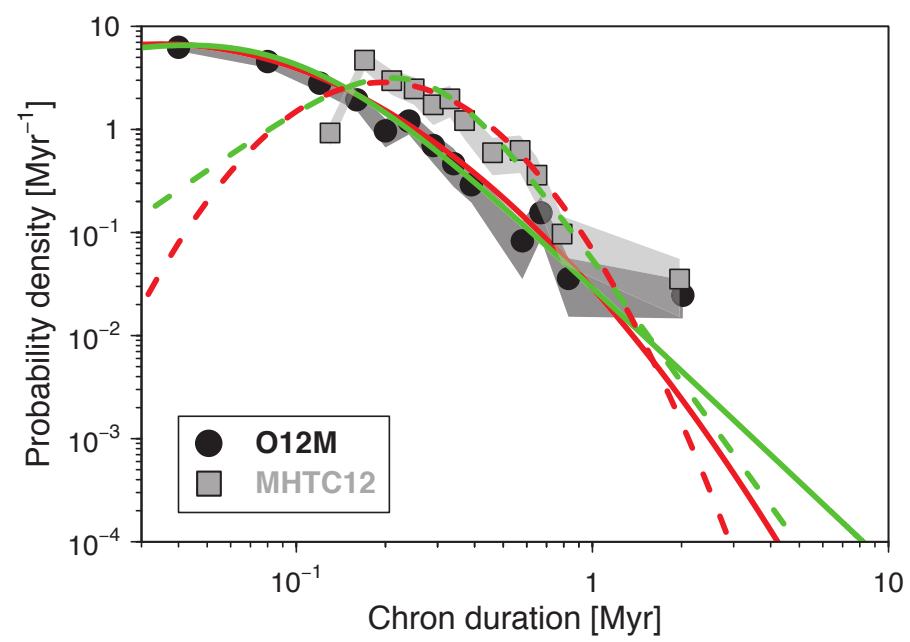

Figure 4.5: Probability densities of geomagnetic chron durations for the Mesozoic time scales MHTC12 and O12M (see legend inset). The gray shaded regions mark the 95\% confidence intervals for the respective binned probability densities. The best-fitting lognormal and log-logistic distributions are shown in red and green respectively (dashed curves for MHTC12 and solid curves for O12M).

capture (Figure 4.5).

In conclusion, the statistic of chron durations during the Mesozoic is sensible to the actual GPTS considered. If the shorter but better constrained reversal chronologies TS10 and MHTC12 are chosen, the log-normal and the log-logistic can be both considered valid models with similar posterior probabilities. This result agrees with our previous analysis of the Cenozoic GPTSs.

The probability density of chron durations for the polarity time scale $\mathrm{O} 12$ (spanning a period up to $171 \mathrm{Ma}$ for a total of 448 chrons) is shown in Figure 4.6 together with the best-fitting log-normal and log-logistic distribution functions. The results previously obtained for shorter GPTSs are confirmed by this longer chronology. Both log-normal and log-logistic distributions present a remarkable agreement with the data at short and intermediate chron durations. However both models largely underestimate the likelihood of the CNS as expected. A positive evidence in favor of the log-normal is suggested by the Bayesian analysis (Figure 4.3). The posterior odds ratio (3.106) of the log-normal to the log-logistic distribution is indeed 5.25 (cf. Table 4.2). However, it remains somewhat difficult to judge which model better describes the data (Figure 4.6). As already discussed above, the larger posterior probability obtained for the log-normal distribution may be an artifact of the short and poorly constrained events included in the oldest part of this chronology.

A final instructive test has been conducted discarding the CNS from $\mathrm{O} 12$ (O12-CNS). The estimated log-normal and log-logistic parameters relative to O12 and to O12-CNS are equal in the limits of statistical errors (Table 4.2). Best-fitting probability densities of chron durations without the CNS are thus practically indistinguishable from those shown in Figure 4.6. Nonetheless, the Bayesian analysis reports a decisive evidence in favor of the log-normal model which has a posterior probability $\mathrm{P}(\log$ Normal $\mid \Delta t)$ of $99.7 \%$. As already discussed analyzing the Cenozoic GPTSs, the superchron exclusion peaks 


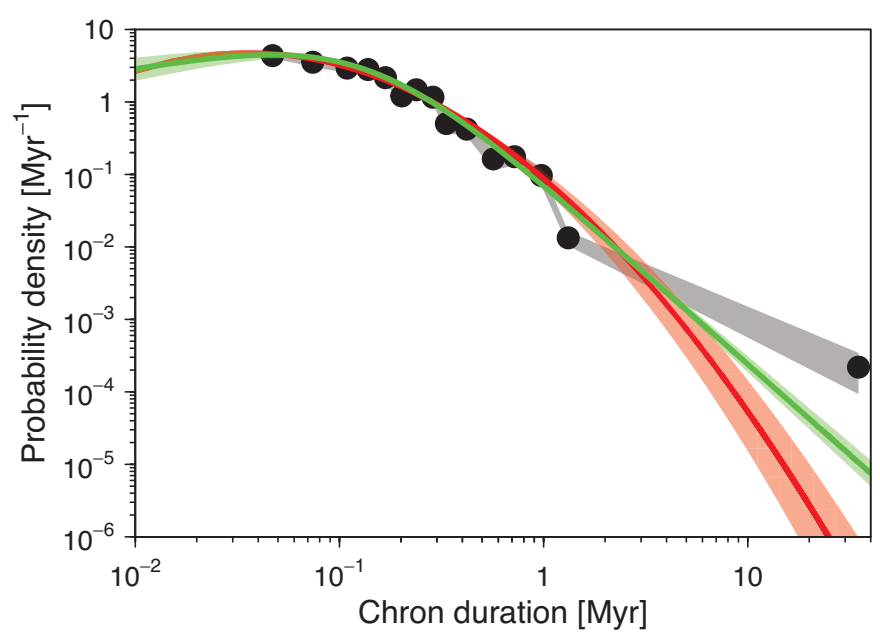

Figure 4.6: Probability density of geomagnetic chron durations relative to the time scale O12 (cf. Table 4.1). The gray shaded region marks the $95 \%$ confidence interval for the binned probability density. Red and green curves show the best-fitting log-normal and log-logistic distributions respectively. Color shaded areas denote the $95 \%$ confidence intervals on the respective distribution parameters estimates.

the likelihood function of the log-normal relative to the log-logistic distribution and this substantially influence the respective posterior model probabilities.

Figure 4.7 shows the posterior densities of the log-normal and log-logistic distribution parameters calculated as in Equation (3.97) for the chron durations $\Delta t$ as recorded by $\mathrm{O} 12 \mathrm{C}$ (panels a and b), MHTC12 (panels c and d) and $\mathrm{O} 12$ (panels e and f). These GPTSs are representative of the Cenozoic, the Mesozoic and the last $171 \mathrm{Myr}$ of geomagnetic history respectively. The log-normal posterior densities are remarkably similar to bivariate normal distributions for all the polarity time scales selected (Figure 4.7 panels $\mathrm{a}, \mathrm{c}$ and e). This indicates that the asymptotic limit of the respective likelihood functions (Equation (3.97) ) is reached and that the priors have only a marginal influence on the posterior distributions. As a consequence, maximum likelihood estimates of the log-normal parameters and the associated standard errors (Table 4.2) determine means and standard deviations of the bivariate normal approximations to the posterior distributions with great precision. The distribution parameters are practically uncorrelated and their correlation coefficients are reported in Table 4.2. The log-normal posterior density relative to the $\mathrm{C}$-sequence presents significantly larger values of the shape parameter $\sigma$ compared to the M-sequence (Figure 4.7, panels a and c). This reflects the larger variability of chron durations during the Cenozoic with respect to the Mesozoic. The extended polarity time scale O12 shows a strong bias of the log-normal posterior density towards lower values of the location parameter $\mu$ due to the large number of short events included (Figure 4.7 panel e).

The posterior densities of the log-logistic parameters $\mathrm{P}(\alpha, \theta \mid \Delta t, \operatorname{LogLogistic})$ cannot be approximated by bivariate normal distributions very accurately (Figure 4.7 panels b, $\mathrm{d}$ and f). Since uniform priors for the parameters $\theta$ and $\alpha$ have been assumed, this effect is due to the data only. Lower $\alpha$ values indicate a lower sample variance and therefore loglogistic PDFs concentrated at lower durations (cf. Figure 3.11). A significant bias of the 
log-logistic posterior density towards lower parameter values is shown for O12 (Figure 4.7. panel f). Once more, this reveals the overwhelming effect of the poorly constrained short chrons recorded by $\mathrm{O} 12$ on the statistic.
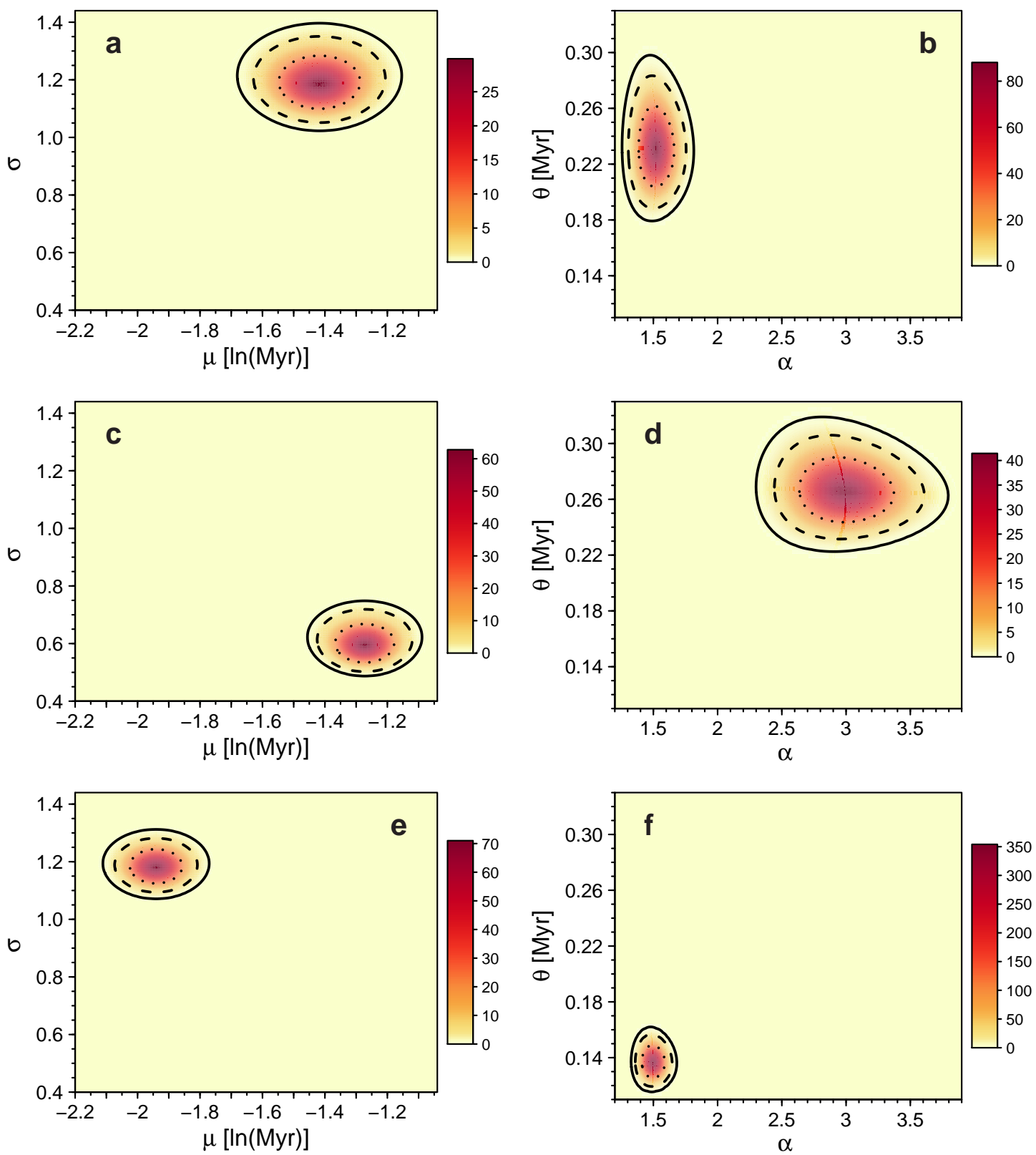

Figure 4.7: Posterior densities of the log-normal (left panels) and log-logistic (right panels) distribution parameters for the chron durations as recorded by the geomagnetic polarity time scales O12C (panels a and b), MHTC12 (panels c and d) and O12 (panels e and f). The selected time scales are representative of the Cenozoic, the Mesozoic and the past $171 \mathrm{Myr}$ respectively (see Table 4.1 for further details). Iso-contour lines mark the $68 \%$, $95 \%$ and $99.7 \%$ highest posterior density intervals.

In conclusion, our analysis suggests that the log-normal and log-logistic distributions describe the geomagnetic polarity interval durations for the last 170 Myr. Ryan and Sarson (2007), comparing a suite of distribution functions in a frequentist framework, also ob- 
tained the most significant fit of chron durations for the log-normal and log-logistic families. As already discussed in Section 3.4.1.5, the authors preferred the former distribution because it naturally arises in simple dynamical systems subjected to the effect of multiplicative noise which have been used to model the occurrence of geomagnetic reversals. An example of this type of stochastic dynamical system is the non-linear mean-field dynamo model of Hoyng et al. (2001). The authors suggested that random perturbations originating in the turbulent core convection may trigger helicity fluctuations (parametrized by the $\alpha$-term in the mean-field dynamo equation) which multiply over time when timestepping the induction equation.

However, our Bayesian analysis shows that the log-normal model is decisively favored over the log-logistic only if the CNS is excluded from the reversal chronologies analyzed. On the other hand, if the superchron (and possibly few other chrons lasting several million years) is regarded as a realization of a single underlying statistical process, the log-logistic would be a more suitable model due to its heavier tail. This is supported by the positive evidence in favor of the log-logistic distribution obtained when including such extreme event in the data sets. The likelihood of the CNS, however, is still largely underestimated by the log-logistic distribution. An alternative description of the long end of paleomagnetic chron durations suggests the use of a pure power-law (see, e.g., Gaffin 1989, Jonkers 2003). As already pointed out above, the main drawback of this distribution is that it poorly describes shorter chron durations where both the log-normal and the log-logistic perform well.

It is therefore difficult to precisely characterize chron durations using distribution functions with stationary parameters. As already discussed in Section 1.4.5 the reversal rate possibly varied during the past $170 \mathrm{Myr}$, decreasing while approaching the CNS and increasing afterwards (see Figure 1.9). Distribution functions with time-dependent parameters have been proposed to take into account such variations (see, e.g., McFadden and Merrill 1984, Constable 2000, and the discussion in Section 1.4.5). These models presuppose the influence of external mechanisms modulating the long-term state of the Earth's core and most often identified of mantle origin (Driscoll and Olson 2011, Biggin et al. 2012). However, some authors suggest that the reversal rate variations may also reflect the dynamics of the strongly non-linear internal dynamo processes (Jonkers 2003, Shcherbakov and Fabian 2012) and arguments in favor of a long-term stationarity have been proposed in several other studies (see, e.g., Hulot and Gallet 2003, Carbone et al. 2006).

Moreover, it is not entirely clear to which extent the poor resolution of the reversal chronologies influences the observed reversal rate (McFadden 1984, Marzocchi 1997). Our analysis uncovers a second type of bias caused by the poor data quality. Short and not fully constrained chrons recorded in the extended Mesozoic time scales O12M and O12 decisively encourage the posterior probability of the log-normal distribution relative to the log-logistic. The superchron and some of the longer events which, on the contrary, tend to favor the log-logistic model are overwhelmed by the large number of short chrons available in these extended chronologies. In order to firmly establish the best alternative between the two models selected by our analysis, it is therefore of fundamental importance to better constrain the shortest geomagnetic polarity interval durations. 



\section{Results from geodynamo simulations}

In this chapter we characterize the statistical properties of reversals and excursions in two geodynamo models. The statistical analysis relies on concepts and methods discussed in Chapter 3. In Section 5.1 we introduce the reversing dynamo models analyzed. The field evolution during typical simulated polarity transitions is described in Section 5.2 Section 5.3 illustrates the algorithm employed to determine the durations of reversals and excursions in the numerical simulations. The statistical analysis of the occurrences and durations of reversals (and excursions) is discussed in Section 5.4 The chapter closes with a comparison of the statistics of the simulated polarity epochs with paleomagnetic reversal chronologies in Section 5.5.

\subsection{Models description and comparison with the Earth}

In order to study the long-term behavior of the dipole field, we selected two reversing dynamo models with different Ekman numbers and Rayleigh numbers tuned to yield Earthlike reversals. Table 5.1 lists the models parameters and the respective estimated values for the Earth's core. Model M1 has a larger Ekman number $E$ of $2 \cdot 10^{-2}$ while the Ekman number of model M2 is twenty times smaller at $E=10^{-3}$. These models have been previously discussed in the literature (Wicht 2005, Wicht et al. 2009).

Due to the actual limitations in the available computational power, present geodynamo models run far away from the Earth's parameter regime and the models analyzed here do not represent an exception (cf. Table 5.1). The most severe discrepancy is in the Ekman number which is at least eleven orders of magnitude larger than the estimated Earth's value. This introduces an excess kinematic viscosity $v$ which acts in damping small spatial scales present at realistic parameter values, but currently unresolvable for practical reasons. Moreover, M1 and M2 have mild Ekman numbers when compared to the most

\begin{tabular}{cccccc}
\hline Model & $E$ & $R a$ & $R a / R a_{c}$ & $P m$ & $P r$ \\
\hline M1 & $2 \cdot 10^{-2}$ & 300 & 2.5 & 10 & 1 \\
M2 & $10^{-3}$ & 500 & 8.9 & 10 & 1 \\
\hline Earth & $10^{-15}-10^{-14}$ & $\gg 1$ & $10^{4} R a_{c}$ & $10^{-6}-10^{-5}$ & $0.1-1$ \\
\hline
\end{tabular}

Table 5.1: List of control parameters for the two dynamo models analyzed. Ekman number $E$, Prandtl number $P r$ and magnetic Prandtl number $P m$ listed for Earth are based on molecular diffusivities. $R a$ indicates the Rayleigh number and $R a_{c}$ its critical value for the onset of convection. 
recent geodynamo models approaching $E=10^{-7}$ (Kageyama et al. 2008). Long temporal integrations, necessary to achieve a statistically significant number of reversals, act as an additional restriction here. Nevertheless, model M2 has the lowest Ekman number among the geodynamo models usually employed in the statistical study of simulated reversals (see, e.g., Lhuillier et al. 2013, Olson et al. 2014).

The vast majority of geodynamo models belongs to a class of solutions where the axial dipole dominates over all the field components but remains largely aligned with the rotation axis (stable dipolar regime). Reversals are therefore expected to be, if occurring at all, extremely rare in such regime. Kutzner and Christensen (2002) demonstrated that increasing systematically the vigor of convection relative to rotational forces, namely increasing the Rayleigh number $R a$ while keeping $E$ constant, leads to a gradual transition from stable dipolar to multipolar solutions (multipolar regime). The range of input parameters in which the dominant dipole field rarely undergoes reversals is quite narrow and lies at the boundary between the dipolar and the multipolar regime (Kutzner and Christensen 2002, Wicht et al. 2009, 2010).

The Earth's core Rayleigh number is hard to estimate but thought to be highly supercritical (Christensen 2011). The numerical dynamos analyzed here are at least two orders of magnitude less supercritical than the Earth (cf. Table 5.1).

The magnetic Prandtl number $P m$ is much smaller than unity for liquid metals. Typical time scales of magnetic diffusion are thus expected to be much lower than viscous ones in the Earth's core (see Section 2.6). Magnetic diffusion is therefore extremely effective and, in order to sustain the magnetic field against ohmic losses, the flow needs to be very turbulent. In numerical models, a self-sustained magnetic field can be achieved only at the price of lower magnetic diffusivities, i.e. for relatively large values of $P m$.

In order to characterize to which extent the reversing behavior of models M1 and M2 resembles the Earth, we defined some dimensionless measures following Wicht et al. (2009). Their values, together with some time-averaged properties, are listed in Table 5.2 .

\begin{tabular}{cccccccccc}
\hline Model & $\bar{\Lambda}$ & $\overline{R m}$ & $\bar{M}$ & $\sigma_{M}$ & $\bar{D}$ & $\tau_{\mathrm{T}}$ & $\overline{|\vartheta|}$ & $\sigma_{|\vartheta|}$ & $\tau$ \\
\hline M1 & 4.6 & 94 & 7.36 & 4.51 & 0.38 & 0.061 & 75 & 15 & $10.8 \cdot 10^{3}$ \\
M2 & 19.0 & 435 & 9.58 & 4.08 & 0.22 & 0.060 & 73 & 14 & $4.6 \cdot 10^{3}$ \\
\hline Earth & $0.1-10$ & $400-2000$ & 7.5 & 1.7 & $<0.6$ & $\sim 0.01$ & 83 & - & $12 \cdot 10^{3}$ \\
\hline
\end{tabular}

Table 5.2: List of time-averaged output parameters and properties for the dynamo models analyzed. Columns from 2 to 5 present time averages of the Elsasser number $\Lambda$, the magnetic Reynolds number $R m$, the dipole moment $M$ and its standard deviation $\sigma_{M}$, and the relative dipole strength at the outer boundary $D$. The time averages are calculated over the total simulation time $\tau$ (last column, in units of the outer core magnetic diffusion time $\left.\tau_{\eta}\right) . \bar{M}$ and its standard deviation $\sigma_{M}$ are given in units of $10^{22} \mathrm{Am}^{2}$. The seventh column shows the relative transitional dipole time $\tau_{\mathrm{T}}$, defined as the fraction of time that the magnetic pole spends further away than $45^{\circ}$ from the closest geographic pole. Columns 8 and 9 are the time averages of the absolute magnetic pole latitude $|\vartheta|$ (in degrees) and its standard deviation $\sigma_{|\vartheta|}$ respectively. Estimates given for Earth are discussed in the text. 
The magnetic pole latitude $\vartheta$ is defined by

$$
\vartheta \doteq \frac{180}{\pi} \arctan \left[\frac{B_{\ell=1, m=1}\left(r=r_{o}, t\right)}{\sqrt{\sum_{m=0,1} B_{\ell=1, m}^{2}\left(r=r_{o}, t\right)}}\right],
$$

where $B_{\ell, m}$ represents the coefficient of order $\ell$ and degree $m$ in the spherical harmonic expansion of the magnetic field (Section 2.8), $r_{o}$ the outer boundary radius and $t$ time. Values of the time-averaged absolute magnetic pole latitude $\overline{|\vartheta|}$ as large as about $75^{\circ}$ show a dipole field closely aligned with the rotation axis in both dynamo models during most of the simulation runs. The temporal variability of the magnetic pole latitude is quantified by the standard deviation of $\overline{|\vartheta|}$ (Table 5.2). The recent global geomagnetic field model for the past $14 \mathrm{kyr}$ of Pavón-Carrasco et al. (2014) reports a time-averaged value of the magnetic north pole latitude of about $83^{\circ}$.

The relative transitional time $\tau_{\mathrm{T}}$ is the time the magnetic pole spends further away than $45^{\circ}$ from the closest geographic pole. This angular threshold has been chosen according to the commonly accepted paleomagnetic practice (see Section 1.4.3). Transitional periods account for roughly $6 \%$ of the total simulation time in both models and are therefore clearly separated from long stable dipole epochs. Reversals can thus be regarded as rare events in these simulations. Considering that a typical geomagnetic reversal transition requires $4-5$ thousand years (Merrill 1998) and that about 300 events have been recorded for the past $160 \mathrm{Myr}, \tau_{\mathrm{T}}$ amounts to no more than a few percent for the Earth. This estimate certainly represents a lower limit because, for example, transitional periods related to excursions have not been considered.

The dipolarity $D$, defined as the square root of the ratio of magnetic dipole energy to the total magnetic energy at the outer boundary

$$
D=\left[\frac{\sum_{m=0,1} B_{l=1, m}^{2}\left(r=r_{o}, t\right)}{\sum_{l} \sum_{m} B_{l, m}^{2}\left(r=r_{o}, t\right)}\right]^{\frac{1}{2}},
$$

provides a quantitative estimate of the degree of dipole dominance. A value of $D \simeq 0.6$ characterizes the recent geomagnetic field given up to degree and order 14 (Maus et al. 2006). Too low dipolarity is characteristic for reversing dynamos at larger Ekman numbers (Wicht et al. 2010). This can be attributed to the smaller influence of rotation at larger Ekman numbers while a geostrophic flow, associated with lower $E$ values, promotes the production of an axial dipole field. The large-scale coherence of the flow is counterbalanced by the increasing influence of inertial forces at larger Rayleigh numbers. In our simulations the time-averaged value of $D$ reaches 0.38 in M1 and 0.22 in M2. As we will demonstrate in the following, the reason of such difference resides not only in the significantly larger $R a$ of model M2, but also in the peculiar behavior of M1 during transitional periods. Obviously, $D$ increases if averaged over stable dipole field polarities only, thus approaching the estimated Earth's value given above.

Time-averaged values of the Elsasser number $\Lambda$ and the magnetic Reynolds number $R m$ are also reported in Table 5.2. The Elsasser number $\Lambda$ measures the ratio of Lorentz to Coriolis forces in the momentum equation (2.74)

$$
\Lambda=\frac{\left\langle B^{2}\right\rangle}{\mu_{0} \rho \Omega}
$$


where $\mu_{0}$ is the vacuum permeability, $\rho$ the fluid density and $\Omega$ the rotation rate. The angular brackets $\langle\cdot\rangle$ denote root mean square (RMS) values calculated over the fluid shell. For an arbitrary scalar quantity $f$, its RMS value is defined as $\langle f\rangle=\left(\widetilde{f^{2}}\right)^{1 / 2}$, where

$$
\widetilde{f}=\frac{3}{4 \pi\left(r_{o}-r_{i}\right)^{3}} \int_{0}^{2 \pi} \int_{0}^{\pi} \int_{r_{i}}^{r_{o}} f(r, \theta, \phi) r^{2} d r \sin \theta d \theta d \phi
$$

represents an average over the fluid shell. In the scaling used here, $\Lambda$ is a dimensionless measure for the mean magnetic field strength in the core (see Section 2.6). M1 and M2 have time-averaged values of $\Lambda$ of 3 and 9 respectively. Since Lorentz and Coriolis forces are expected to be of the same order in the Earth's core (magnetostrophic balance), the Elsasser number is thought to be of order one. Reasonable estimates of the magnetic field strength in the core confirm an Elsasser number in the range 0.1 - 10 (Christensen et al. 2009).

The field strength has been rescaled to dimensional values by means of (5.3) assuming the Earth's rotation rate $\Omega=7.292 \cdot 10^{-5} \mathrm{rad} / \mathrm{s}$ and a mean core density $\rho=1.1 \cdot 10^{4} \mathrm{Kg} / \mathrm{m}^{3}$. Table 5.2 lists the rescaled values of the time-averaged dipole moment $\bar{M}$ for the dynamo models analyzed here. As already discussed in Section 1.4.3, paleointensity measures provide a mean (virtual) axial dipole moment of $(7.5 \pm 1.7) \cdot 10^{22} \mathrm{Am}^{2}$ during the Brunhes chron (see also Figure 1.4). Continuous global geomagnetic field reconstructions for the past $10 \mathrm{kyr}$ (Korte et al. 2011) and 14 kyr (Pavón-Carrasco et al. 2014) agree with this estimate. The dipole moment inferred from such models ranges between a minimum value of $4.5 \cdot 10^{22} \mathrm{Am}^{2}$ and a maximum of $11.0 \cdot 10^{22} \mathrm{Am}^{2}$. Our dynamo simulations show time-averaged dipole moments falling within this range.

The magnetic Reynolds number $R m$ represents the ratio of magnetic advection to magnetic diffusion in the induction equation (2.71) and is defined as

$$
R m=\frac{\langle u\rangle d}{\eta}
$$

where $u$ is the fluid velocity, $d$ the outer shell thickness and $\eta$ the magnetic diffusivity. A lower bound for the Earth's $R m$ can be estimated from the westward drift velocity of the non-dipole field which is on average approximately 0.18 degree/year (Bullard et al. 1950). Assuming that such motion reflects typical flow velocities at the top of the Earth's outer core, $R m$ can be estimated to be around 400. Christensen and Tilgner (2004), analyzing a suite of geodynamo models, predicted a higher $\mathrm{Rm}$ of 1200 for the Earth's core. Recently revised estimates for the electrical conductivity of the fluid core (Pozzo et al. 2012) would suggest a yet larger value of $R m \simeq 2000$. The magnetic Reynolds number of model M2 is somewhat more realistic at $R m=435$, while the value of $R m=94$ for model M1 is on the low side. $R m$ could be increased, for example, considering a larger Rayleigh number, but this would drive both dynamos into the multipolar regime where the dipole looses its dominance and reverses more or less continuously.

$R m$ can also be interpreted as the ratio of the magnetic diffusion time of the outer core $\tau_{\eta}=d^{2} / \eta$ to the advection time scale $\tau_{u}=d /\langle u\rangle$ (convective turnover time). As mentioned above, dimensionless times in the numerical simulations are rescaled to real values using $\tau_{\eta}$. However, if the typical time scales of magnetic field variations studied here are ruled by the vigor of convection rather than by diffusive effects, the rescaled 
times result to be overestimated. Moreover, there are evidences that for many dynamo processes the advective time may play a more important role (see, e.g., Olson et al. 2012). This could be accounted for by dividing the dimensional times given in the following by a factor $R m_{\text {Earth }} / R m$. Using $R m_{\text {Earth }} \simeq 2000$ suggests correction factors of about 4.6 for M2 and 21 for M1.

In order to achieve a significant number of reversals, models M1 and M2 have been integrated for several thousand magnetic diffusion times. Figures 5.1 and 5.2 illustrate time series of the dipole tilt angle (or dipole colatitude) $\Theta=90^{\circ}+\vartheta$ and the total dipole moment $M$ (in rescaled units) for M1 and M2 respectively. The higher temporal variability of M2 with respect to M1 is evident. The former dynamo model is indeed characterized by abrupt reversal transitions occurring with a higher frequency compared to M1. The stronger convective driving of M2 is responsible for such enhanced reversal activity. Periods of diminished dipole intensity nicely correlate with large dipole tilts in model M2. This is not always the case in M1 where longer periods of weak dipole intensity may present stages of stable dipole field polarity.

Figure 5.3 shows histograms of the dipole tilt angle $\Theta$, dipole moment $M$ and dipolarity $D$ for the dynamo models analyzed. Distributions of transitional times (namely periods characterized by $45^{\circ} \leq \Theta \leq 135^{\circ}$ ) are illustrated in red. Opposite field polarities are roughly equally represented in both dynamo models as expected (Figure 5.3, panels $a$ and $b$ ). The induction equation (2.93b) is indeed invariant under the transformation $\mathbf{B} \rightarrow-\mathbf{B}$ meaning that the same type of dynamo mechanism operates independently of the sign of the magnetic field. However, a certain imbalance between the two polarities still persists even after several thousand magnetic diffusion times.

Dipole moment distributions of the two models are markedly different (Figure 5.3, panels c and d). M2 shows a unimodal distribution with its mode at about $11 \cdot 10^{22} \mathrm{Am}^{2}$. Transitional periods seem to cause a hump at low $M$ values which is suggestive of the presence of a low dipole stage distinct from the leading stable field phase. As already noticed above, weak dipole moments nicely correlate with transitional periods and this is quantified by a Pearson correlation coefficient between $M$ and the absolute magnetic pole latitude $|\vartheta|$ of 0.52 (see Table 5.3).

On the contrary, the time spent in weak dipole stages is comparable to that of the stronger and stable dipole field phases in model M1 (Figure 5.3, panel c). Furthermore, low dipole intensities correlate not entirely with transitional periods (highlighted in red) but even more likely with stable field polarities. Consistently, poor deviations of the dipole from the rotation axis are also observed at low $D$ values (Figure 5.3 panel e). Model M2, which does not display such behavior, presents a fraction of low dipole tilts having small dipolarity which is relatively modest (Figure 5.3, panel f).

Note also that the dipolarity distribution of transitional periods of M1 shows a heavier tail compared to M2. The former model, contrary to the latter, presents therefore significant dipolar contributions relative to the total field strength during weaker dipole periods. This is confirmed by a somewhat lower correlation coefficient between dipole moment and dipolarity obtained for M1 (see Table 5.3). The reasons for the observed differences between the two dynamo models at low field strengths will be discussed in Section 5.2

Before analyzing in detail the temporal behavior of the two dynamos during reversal transitions, we illustrate typical magnetic and flow field solutions at times representative of stable polarity epochs, i.e. presenting a relatively strong dipole field closely aligned 

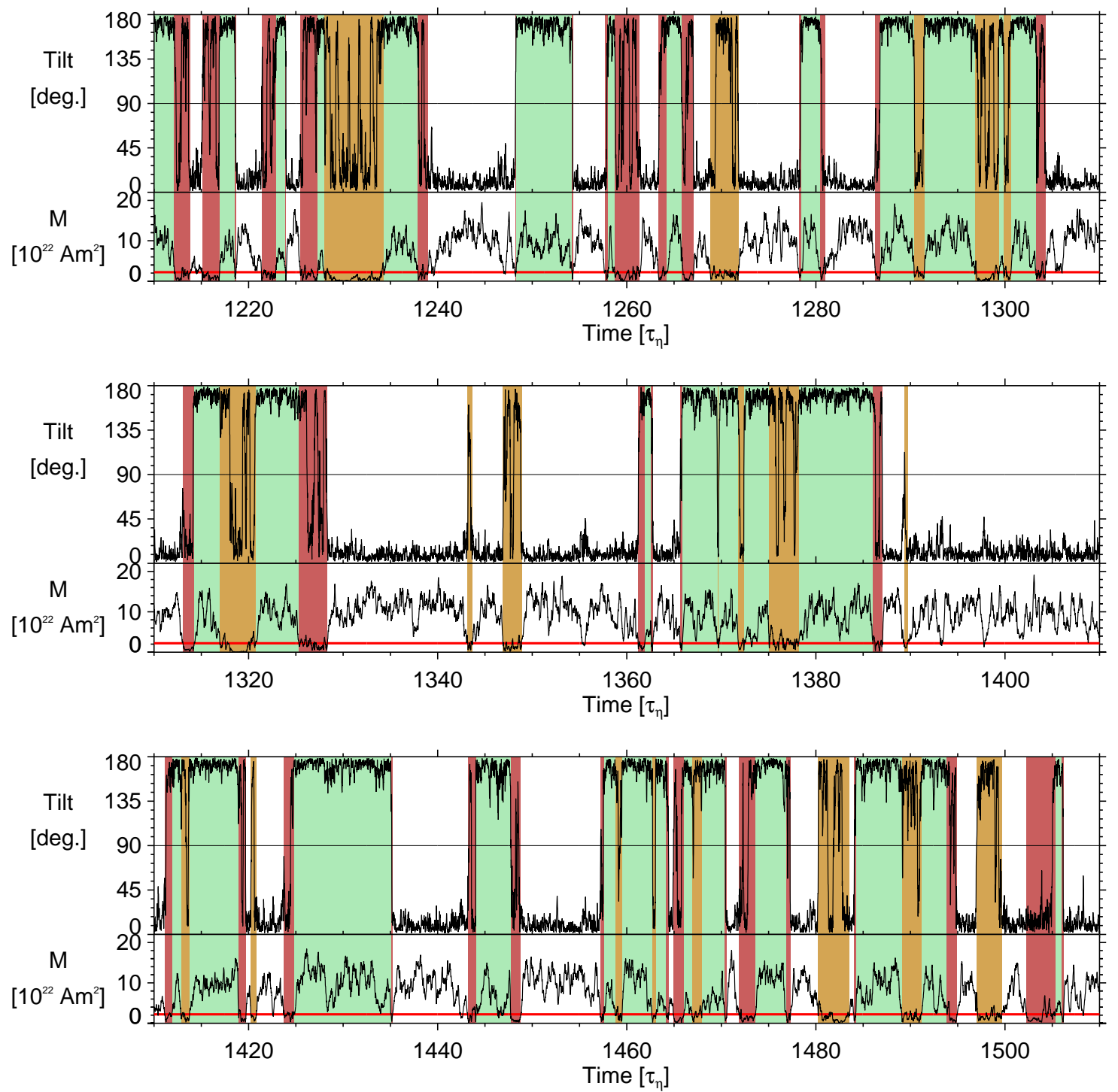

Figure 5.1: Time series of dipole tilt angle (upper sub-panels) and dipole moment $M$ (lower sub-panels) for dynamo M1. Opposite stable polarities are marked in white and green. Red and orange background colors highlight reversals and excursions respectively. The horizontal red line marks the critical value of $M$ used to define the events durations and equals $30 \%$ of its time-averaged value. Events spaced by less than $0.4 \tau_{\eta}$ have been melted and only excursions crossing the equator are shown (see Section 5.3 for a detailed description of the algorithm employed). 

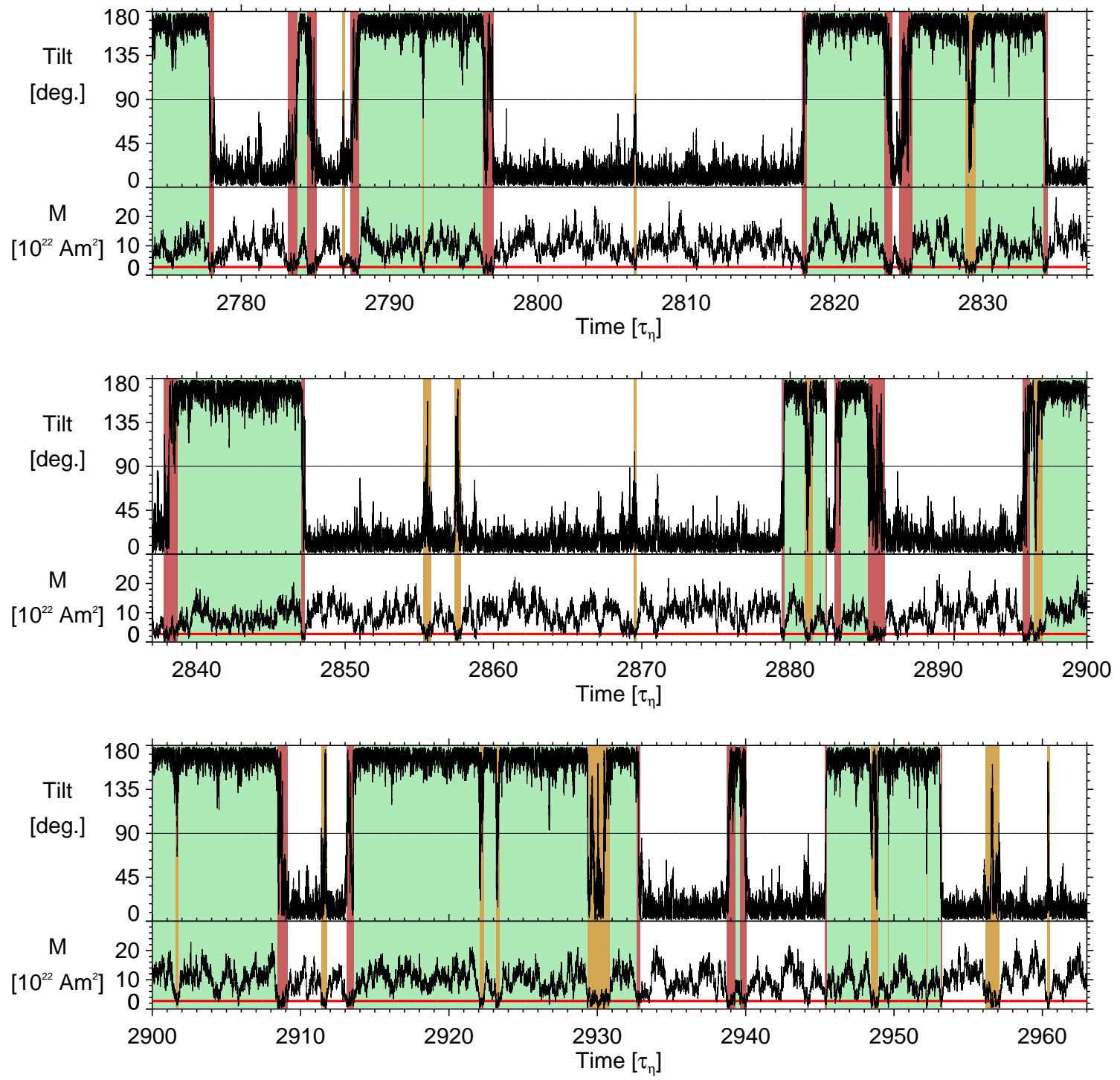

Figure 5.2: Time series of dipole tilt angle (upper sub-panels) and dipole moment $M$ (lower sub-panels) for dynamo M2. Opposite stable polarities are marked in white and green. Red and orange background colors highlight reversals and excursions respectively. The horizontal red line marks the critical value of $M$ used to define the events durations and equals $30 \%$ of its time-averaged value. Events spaced by less than $0.2 \tau_{\eta}$ have been melted and only excursions crossing the equator are shown (see Section 5.3 for a detailed description of the algorithm employed). 

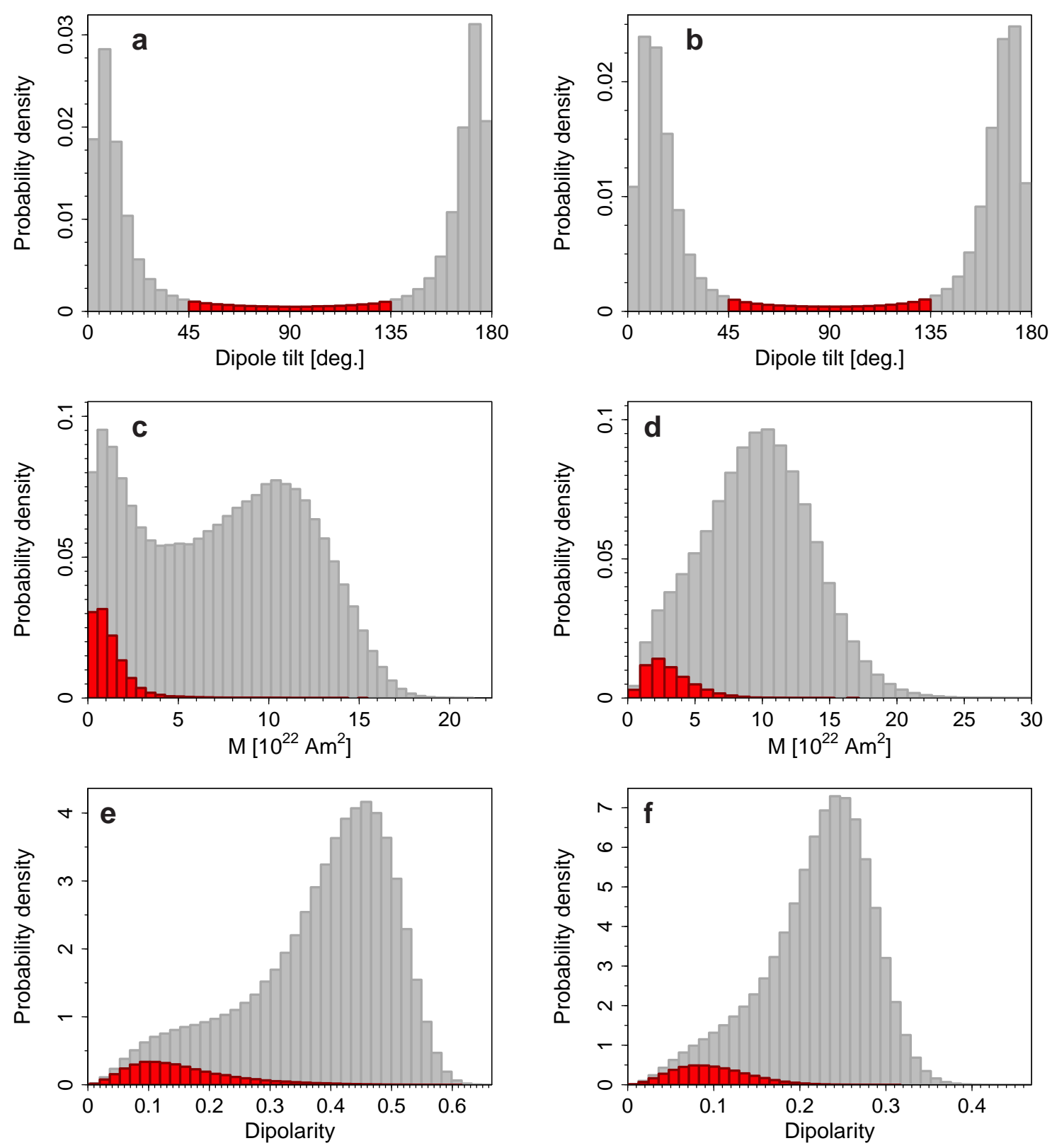

Figure 5.3: Histograms of dipole tilt angle $\Theta$ (panels a and $\mathrm{b}$ ), total dipole moment $M$ (panels c and d) and dipolarity $D$ (panels e and f) for models M1 (left column) and M2 (right column). Transitional times, namely periods with $45^{\circ} \leq \Theta \leq 135^{\circ}$, are highlighted in red.

with the rotation axis. At the selected times, the dipolarity value is $D=0.21$ for M1 and $D=0.10$ for $\mathrm{M} 2$, while tilt angles $\Theta$ are $5^{\circ}$ and $15^{\circ}$ for $\mathrm{M} 1$ and $\mathrm{M} 2$ respectively. Figure 5.4 shows the corresponding snapshots of the radial field component at the outer boundary $B_{r}\left(r_{o}, \theta, \phi\right)$ and of the radial velocity at mid-depth $u_{r}\left(r_{i}+d / 2, \theta, \phi\right)$.

In both dynamo models, the radial magnetic field at the outer boundary is characterized by polarity flux-patches of opposite polarity in the two hemispheres. Such magnetic structures are more small scaled for M2 as expected and are due to its lower Ekman number and the more vigorous convective driving. The stronger flux-patches are distributed 


\section{model M1}
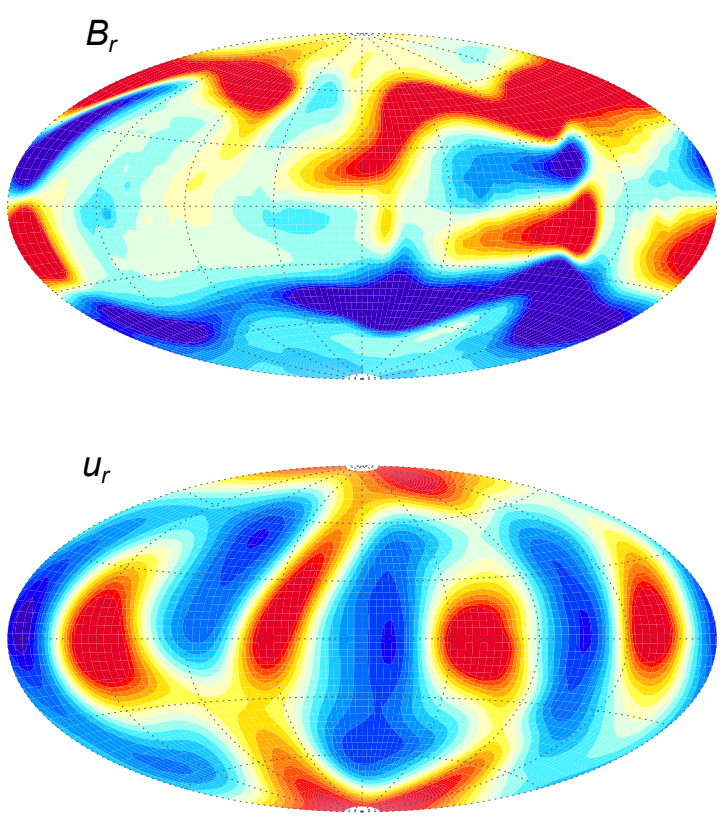

\section{model M2}
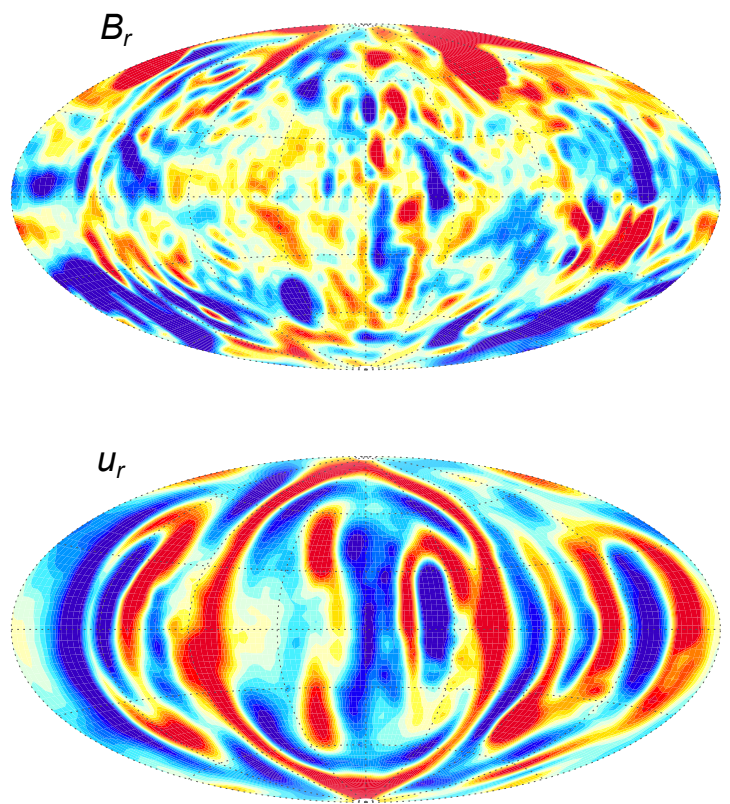

Figure 5.4: Snapshots of the radial field component $B_{r}$ at the outer boundary (first row) and radial flow velocity $u_{r}$ at mid-depth (second row) for models M1 and M2 representative of stable dipolar configurations. Blue and yellow/red colors indicate inward and outward directed fields respectively. Color contours are scaled according to the absolute maximum of the respective fields. The absolute maximum magnetic field strength is $B_{r}=16.2 \sqrt{\mu_{0} \eta \rho \Omega}$ for M1 and $B_{r}=16.6 \sqrt{\mu_{0} \eta \rho \Omega}$ for M2; the absolute maximum velocity field strength is $u_{r}=277.0 \eta / d$ for M1 and $u_{r}=1060.1 \eta / d$ for M2.

at mid to high latitudes in M1, but tend to cluster towards somewhat higher latitudes in M2. These patches are responsible of the strong axial dipole contribution to the total field strength in both cases. Furthermore, less intense low-latitude patches, generally of the opposite sign of the leading polarity in each hemisphere, are present. The magnetic field in M1 is strongly equatorial anti-symmetric, while M2 does not show the same degree of anti-symmetry. The higher $R a$ of the latter model is the main reason for the spatial symmetry breaking (Wicht 2005).

Radial velocity fields at mid-depth, shown in the bottom row of Figure 5.4, illustrate the pattern of convection. The flow is clearly organized in relatively thin columns elon-

\begin{tabular}{lcr}
\hline Model & $r(M,|\vartheta|)$ & $r(M, D)$ \\
\hline M1 & 0.56 & 0.77 \\
M2 & 0.52 & 0.91 \\
\hline
\end{tabular}

Table 5.3: Pearson correlation coefficients $r$ for the dipole moment $M$ with the absolute magnetic pole latitude $|\vartheta|$ (second column) and with the dipolarity $D$ (third column) for the two dynamo models analyzed. 
gated along the rotation axis in model M2. This is the preferred structure of convection in a rapidly rotating spherical shell. Even though the Ekman number is not very low, the models analyzed here are also moderately supercritical (see Table 5.1) and therefore the ordering influence of Coriolis forces still dominates over inertia. However, the larger Ekman number of M1 results in a flow which do not seem properly geostrophic.

As already mentioned above, the dipole field is mainly formed by the superposition of the bundles of concentrated radial flux at high latitudes (Figure 5.4 top panels). As described in previous studies (Christensen et al. 1998, Olson et al. 1999), a significant secondary flow along and into the convective columns is mainly responsible for such flux concentrations. This can be seen comparing the radial component of the magnetic field $B_{r}$
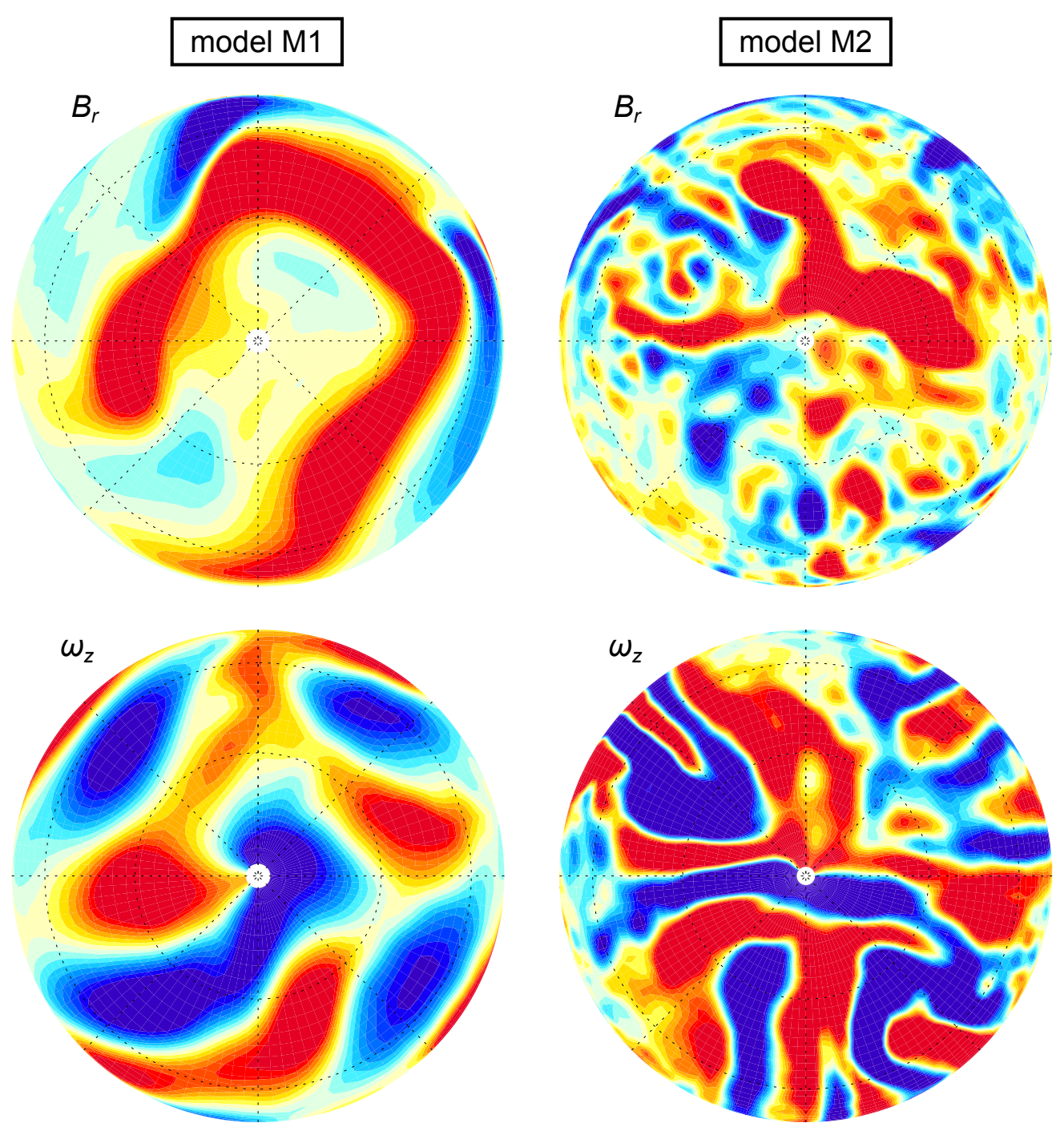

Figure 5.5: Snapshots of the radial magnetic field component $B_{r}$ at the outer boundary (upper panels) and vertical component of the flow vorticity $\omega_{z}$ at $r=0.9 r_{o}$ (lower panels) for model M1 (left panels) and M2 (right panels). One hemisphere is shown with the north pole in the center of the projection. Blue and yellow/red colors indicate inward (negative) and outward (positive) magnetic field (vorticity). Color contours are scaled with the absolute maximum of the respective fields. 
at the outer boundary and the vertical component of the flow vorticity $\omega_{z}$ just below the outer boundary as shown in Figure 5.5. A good correlation exists between positive vorticity (cyclones) and concentrations of radial magnetic field flux. The significant secondary flow along the axis of convection columns directed towards the equator in cyclones and away from the equator in anticyclones results, at the upper and lower ends of the convection columns, in a horizontal flow convergence from negative to positive vortices. Assuming the frozen-flux approximation to be valid $(R m \gg 1)$, these flows advect field-lines towards the cyclones and yield to the respective field concentrations at high latitudes.

\subsection{Field evolution during polarity transitions}

As pointed out in the previous section, the two geodynamo models analyzed strongly differ in the time spent with low dipolar energy. Field polarity transitions are therefore expected to differ. In this section we closely analyze the field evolution during transitional periods in both dynamo models.

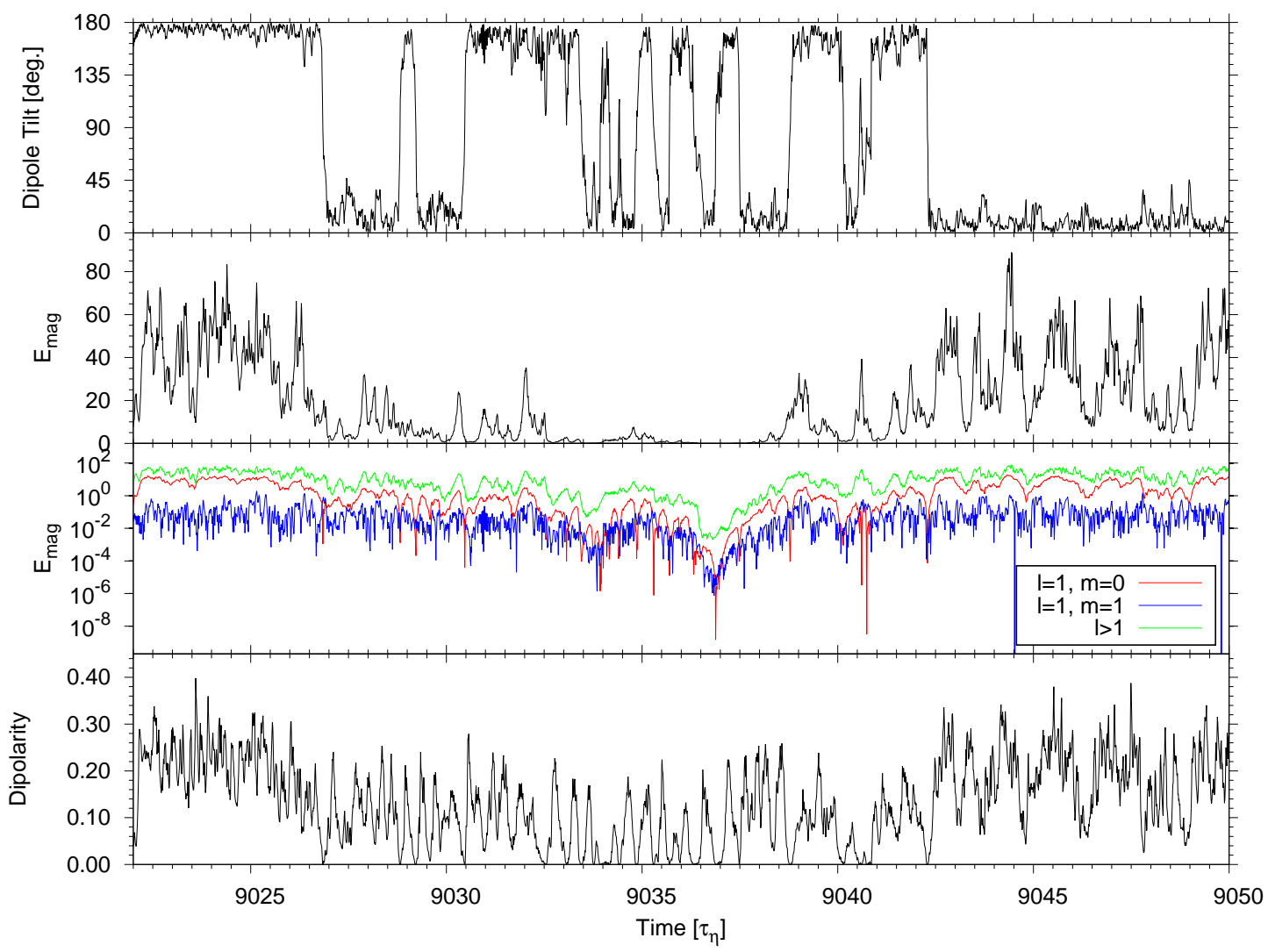

Figure 5.6: Time series of dipole tilt angle $\Theta$ (first panel), total magnetic energy at the outer boundary (second panel), magnetic energy of the axial dipole $(\ell=1, m=0)$, equatorial dipole $(\ell=1, m=1)$ and multipolar components $(\ell>1)$ at the outer boundary (third panel) and dipolarity $D$ (fourth panel) for a long lasting polarity transition in model M1.

Figure 5.6 and Figure 5.7 show two particularly slow polarity transitions occurring in models M1 and M2 respectively. In both dynamos, the dipole experiences significant 
angular deviations from the rotation axis when the overall magnetic field is weak (first and second panels). The axial dipole, the equatorial dipole and all the higher multipolar field contributions of M1 decay when entering the weak field epoch (Figure 5.6, third panel). It seems that the dynamo mechanism stops operating for a certain time until it suddenly restarts. The dipolarity $D$ oscillates eradicably during the weak field stage (Figure 5.6, fourth panel) and the dipole shows, consequently, a succession of normal and reverse field polarities. It is the coherent evolution of the axial and equatorial fields which causes the magnetic pole to remain closely aligned with the rotation axis during most of the transitional phase. These periods significantly populate the low end of the dipole moment distribution as already observed in Figure 5.3 (panel c). This behavior also explains the high values in the dipolarity distribution obtained for transitional periods (cf. Figure 5.3. panel e).

On the contrary, M2 shows what is regarded a more typical behavior of reversing geodynamo models. Figure 5.7 displays a weak field episode mainly due to a decrease in the axial dipole intensity, while the equatorial dipole and the higher multipolar contributions seem to remain rather unaffected (third panel). The weak axial dipole allows for transitional pole positions which may be interpreted as a succession of excursions preceding the polarity change. This event is, however, exceptionally long (with a duration of about $30 \tau_{\eta}$ ) and uncovers one of the most complex field transitions.

Though the type of transition analyzed here for model M1 constitutes a significant

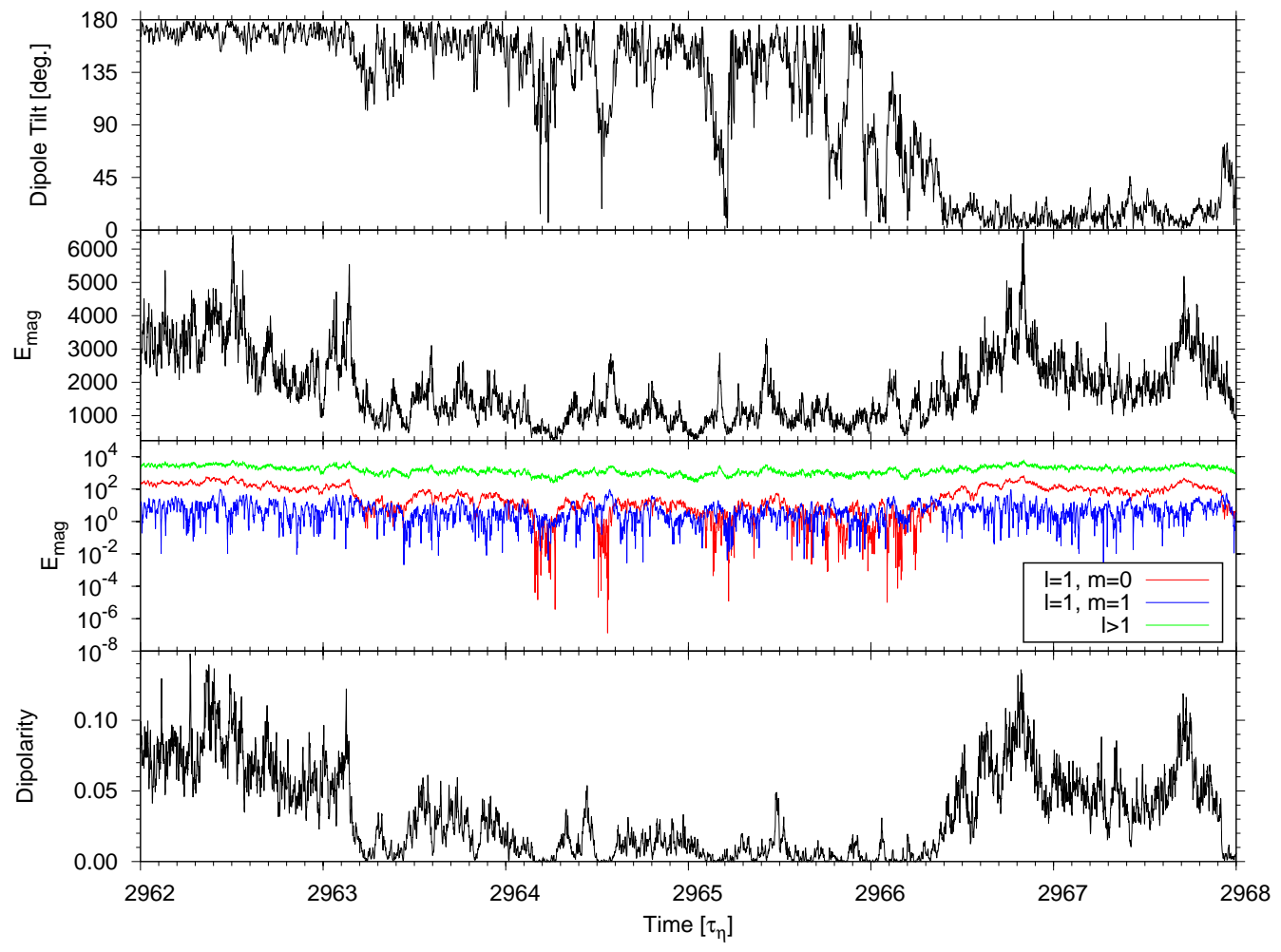

Figure 5.7: Time series of dipole tilt angle $\Theta$ (first panel), total magnetic energy at the outer boundary (second panel), magnetic energy of the axial dipole $(\ell=1, m=0)$, equatorial dipole $(\ell=1, m=1)$ and multipolar components $(\ell>1)$ at the outer boundary (third panel) and dipolarity $D$ (fourth panel) for a long lasting reversal in model M2. 
fraction of the total number of reversals, it does not represent the rule as can be seen from the time series in Figure 5.1. Reversals with a field evolution similar to those observed for M2 are indeed also common in M1. Polarity transitions characterized by a decay in all the magnetic field components are nevertheless sufficient to affect the reversal statistic of model M1.
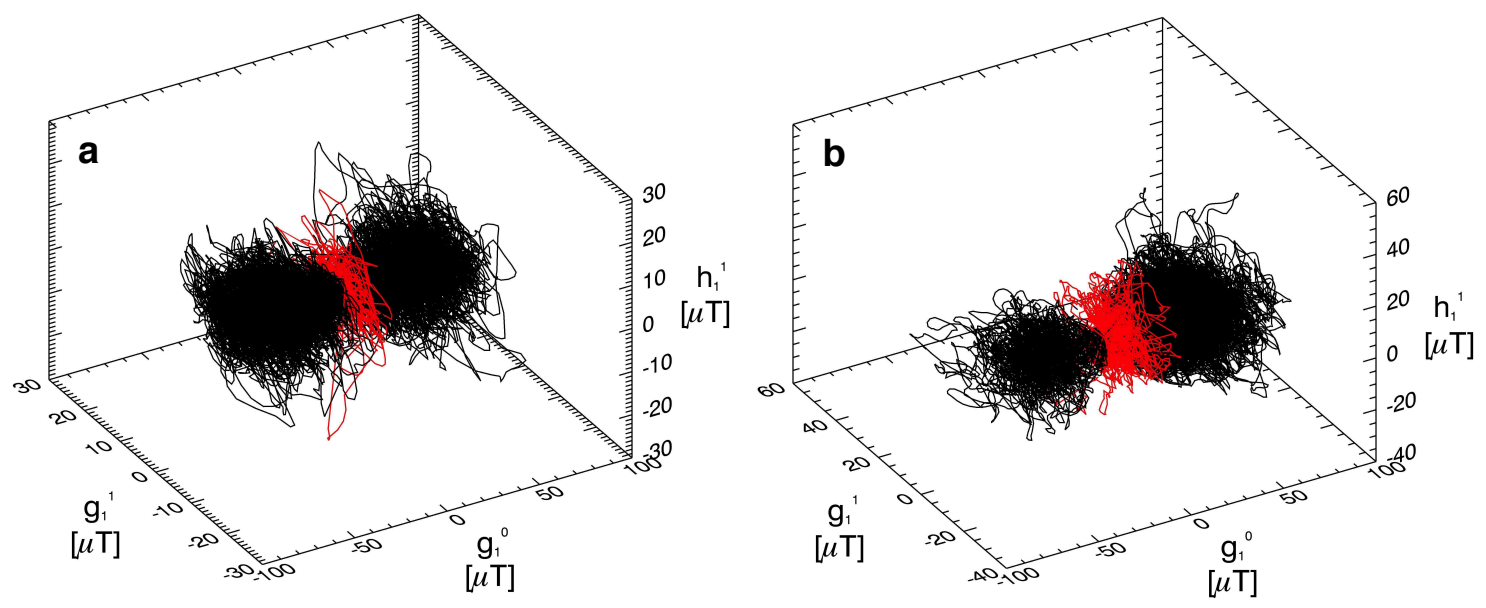

Figure 5.8: Dipole field trajectories in the phase space constituted by the Gauss coefficients $g_{1}^{0}, g_{1}^{1}$ and $h_{1}^{1}$ for models M1 (panel a) and M2 (panel b). For each model, the time interval analyzed is of about 80 outer core magnetic diffusion times and contains several reversals. Red highlights the transitional trajectories where the magnetic pole is more than $45^{\circ}$ away from the rotation axis.

The different evolution of the dipole field for the two dynamo models can be described, over longer time spans, in the phase space constituted by the three Gauss coefficients $g_{1}^{0}$, $g_{1}^{1}$ and $h_{1}^{1}$. Figure 5.8 depicts the phase space trajectories of the dipole field during several tens of magnetic diffusion times for both dynamo models. The transitional field polarities, namely periods defined by a dipole tilt $45^{\circ} \leq \Theta \leq 135^{\circ}$, are marked in red. Both dynamos show two symmetric states dominated by the axial dipole component $g_{1}^{0}$ which represent the two opposite stable field polarities (Figure 5.8, black curves). Transitional dipole tilts are achieved by a significant weakening of $g_{1}^{0}$ in both cases. Model M1, contrary to M2, shows a high density of non-transitional trajectories in the region where the total dipole intensity is low. This highlights, once more, the fact that relatively long periods of weak dipole intensity characterized by mild dipole tilts are not isolated phenomena in M1 but are rather part of its dynamical behavior. On the contrary, transitional trajectories are more clearly correlated with low dipole field stages in model M2. These transitional phases are likely caused by major weakenings of the axial dipole, while the equatorial components $g_{1}^{1}$ and $h_{1}^{1}$ show variations not significantly different from those experienced during the stable field polarities (Figure 5.8, panel b).

Paleomagnetic findings suggest that the behavior of model M2 is more realistic for Earth where too long periods of very low field intensity seem untypical and low dipole moments highly correlate with large angular deviations of the magnetic pole (see, e.g., Merrill and McFadden 1999, Valet et al. 2005, and the discussion in Section 1.4.3). 


\subsection{Definition of events and filtering algorithm}

The algorithm employed for timing the duration of reversals and excursions in the numerical simulations is described in this section. Empirical working definitions commonly used in paleomagnetism serve as a guidance here (see Chapter 1).

Since reversals and excursions are both periods of weak (global) dipole intensity, a natural way to time their start and end is to rely on the dipole moment variations. The $i$-th event starts at the time $t_{i}^{(s)}$ when the dipole moment $M$ decreases below a critical value $M_{C}, t_{i}^{(s)}=t\left(M \leq M_{C}\right)$, and it ends once $M$ subsequently exceeds this value at the time $t_{i}^{(e)}=t\left(M>M_{C}\right)$. This event is defined as a reversal if the field polarities at its starting and ending times are opposite and is an excursion otherwise. In the following, $\mathcal{M}_{C}$ denotes the critical value of the dipole moment $M_{C}$ normalized with the time-averaged dipole moment $\bar{M}$.

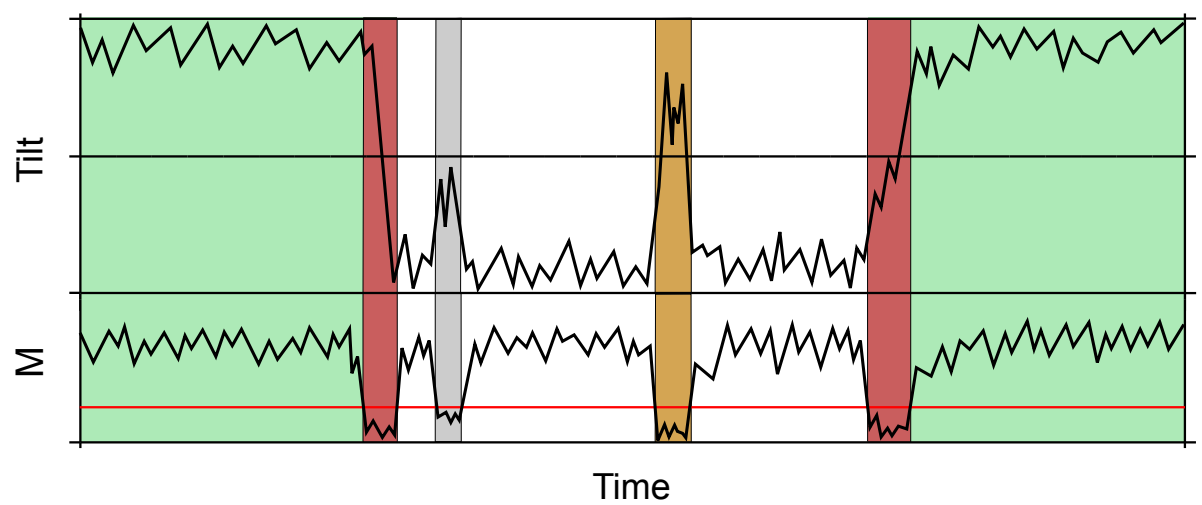

Figure 5.9: Sketch of dipole tilt (upper sub-panel) and dipole moment (lower sub-panel) time series illustrating the different types of events defined. The opposite stable field polarities are marked in white and green. Red, orange and gray backgrounds highlight reversals, grand excursions and dipole dips respectively. The horizontal red line marks the critical dipole moment $M_{C}$ used to define the event durations (see the main text for details).

Figure 5.9 sketches a time series of the dipole tilt and dipole moment illustrating the different types of events defined. Event durations $\Delta t_{i}=t_{i}^{(e)}-t_{i}^{(s)}$ are marked with different colors according to the event type. Reversal and excursion durations are highlighted in red and orange respectively. Let $R_{i}$ and $E_{i}$ denote the $i$-th reversal and excursion respectively and $\Delta t_{i}^{R}$ and $\Delta t_{i}^{E}$ their durations. The duration of the $i$-th stable polarity interval (SPI) of the field $\Delta t_{i}^{P}$ is defined as the time difference between the starting time of the $i$-th reversal and the end of the previous one, thus excluding the two bounding reversal transitions $\Delta t_{i}^{R}$ and $\Delta t_{i+1}^{R}$. Opposite SPIs are marked in white and green in Figure 5.9.

The last type of event defined is called dipole dip and an example is shown in gray in Figure 5.9. Dipole dips are characterized by a decrease in dipole moment comparably shallower than those experienced during reversal transitions. Even though dipole dips promote significant deviations of the dipole from the rotation axis, they usually do not reach the opposite hemisphere. Wicht (2005) and Wicht et al. (2009) reported that large drops in dipole strength are required to allow tilt angles that venture into the opposite polarity and which are detectable as global events in their numerical simulations. This is also the 
case for the two geodynamo models analyzed here where reversals are characterized by a decrease in dipole moment to at least $20 \%$ of its time-averaged value (see Section 5.3.1).

To keep the distinction between excursions and dipole dips clear, we introduce further requirements for the former type of events. In order to regard an event as an excursion, we additionally require that (a) the dipole moment has to drop below a critical value $\mathcal{M}_{C}^{\mathcal{E}}$ (in units of $\bar{M}$ ) and (b) the magnetic pole reaches the opposite hemisphere at least once during the event. We refer to this subset of excursions to as grand excursions.

Short term variability of the dipole moment due to secular variation activity around the threshold $\mathcal{M}_{C}$ frequently leads to undesirable brief events which are typically closely spaced in time. In order to avoid such situation, we demand that events must be separated by a stable time interval (namely a period where $M>M_{C}$ ) lasting at least $T_{S}$. In other words, this filter attempts to smooth out the fastest dipole moment variations. In the following, $\mathcal{T}_{S}$ denotes the stable period $T_{S}$ in units of the magnetic diffusion time $\tau_{\eta}$. Events occurring closer than $\mathcal{T}_{S}$ are merged together into a single reversal or excursion according to the following rules. The possible combinations of successive events are:

(a) $R_{i}$ and $R_{i+1}$,

(b) $E_{i}$ and $R_{j}$ (or vice versa),

(c) $E_{i}$ and $E_{i+1}$

and they are sketched in the respective panels of Figure 5.10.

After the application of the temporal filter, the combination (a) gives a single excursion beginning at the starting time of $R_{i}$ and stopping at the ending time of $R_{i+1}$ (Figure 5.10, panel a). The brief mild recovery of the dipole moment is not deemed significant enough to classify the respective period as a SPI. Discarding two successive reversals has therefore the net effect of producing a longer SPI, obtained by merging the two adjacent ones, punctuated by an excursion.

Panel $b$ of Figure 5.10 shows the effect of the temporal filter on the event combination (b). The excursion $E_{i}$ is now regarded as part of a longer reversal beginning at the starting time of $E_{i}$ and ending when $R_{j}$ stops. The large dipole tilt variability preceding the reversal is thus considered as a single weak field period leading to a longer reversal transition.

Finally, the combination (c) considers two successive excursions temporally spaced by less than $\mathcal{T}_{S}$. The two successive excursions $E_{i}$ and $E_{i+1}$ are now regarded as a single excursion which comprises the two events (Figure 5.10, panel c). The dipole intensity between the two initial events appears indeed too low to account for truly stable dipole directions during such period.

Several pitfalls can occur when defining reversals and excursions via $\mathcal{M}_{C}$ and $\mathcal{T}_{S}$, in particular for extreme parameter combinations. Since these problems may bias the statistics of event durations, we discuss the most relevant ones here. Figure 5.11 shows illustrative examples of four potential problems. Solid and dashed lines in the dipole tilt time series illustrate the two possible polarity alternatives after a period of weak dipole intensity. Blue, green and orange background colors highlight event durations determined with different choices of $\mathcal{M}_{C}$ (horizontal lines). The first problem, denoted as P1 in Figure 5.11 is an over-estimation of the event duration. When $\mathcal{M}_{C}$ is chosen too large (blue horizontal line) we may regard insignificant dipole moment variations as start or end of a 
reversal (or an excursion) should these happen closer than $\mathcal{T}_{S}$ to the 'true' event. The impact of this problem increases with $\mathcal{T}_{S}$ and therefore particularly affects the combination of large $\mathcal{M}_{C}$ and long $\mathcal{T}_{S}$.

If $\mathcal{M}_{C}$ is chosen too low (orange horizontal line) we may wrongly classify periods where the dipole moment $M$ recovers only mildly as a SPI. This problem P2, illustrated in the top right panel of Figure 5.11, increases the likelihood for short SPIs and also artificially separates a reversal (solid dipole tilt curve) into a shorter reversal and a shorter excursion. It may even separate a major excursion (dashed dipole tilt curve) into two shorter reversals. P2 therefore modifies the statistic of event durations. The likelihood for

\section{a. Successive Reversals}
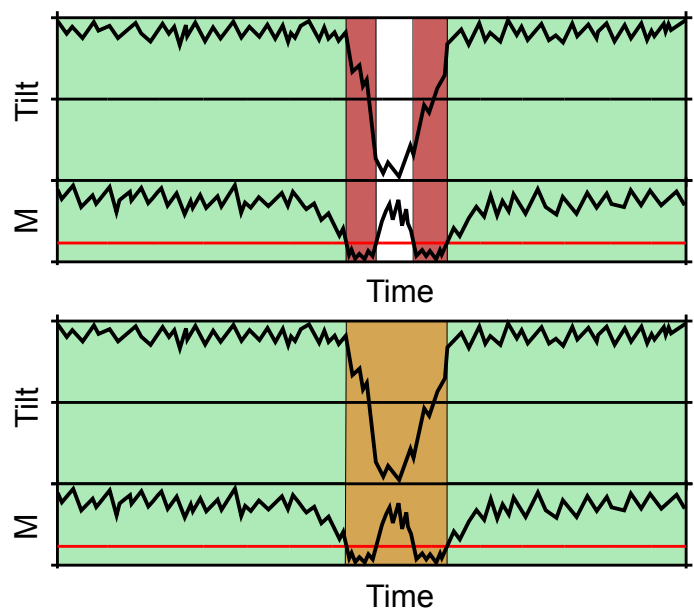

b. Excursion and Reversal
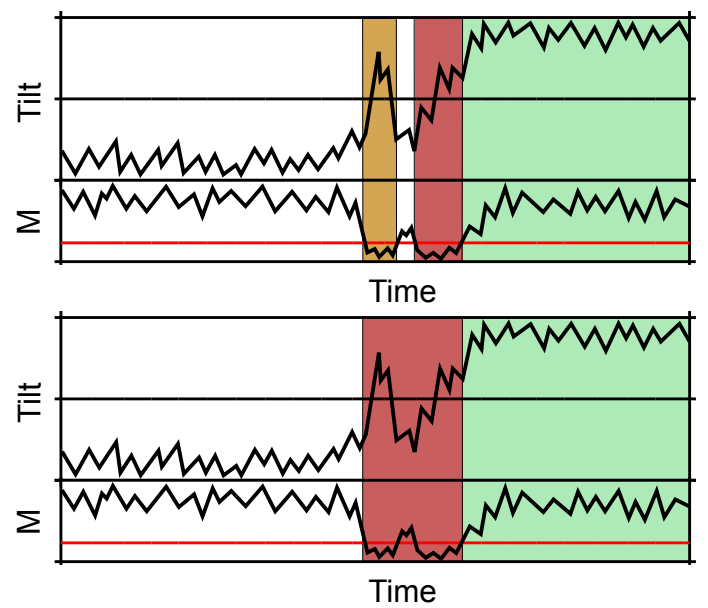

c. Successive Excursions
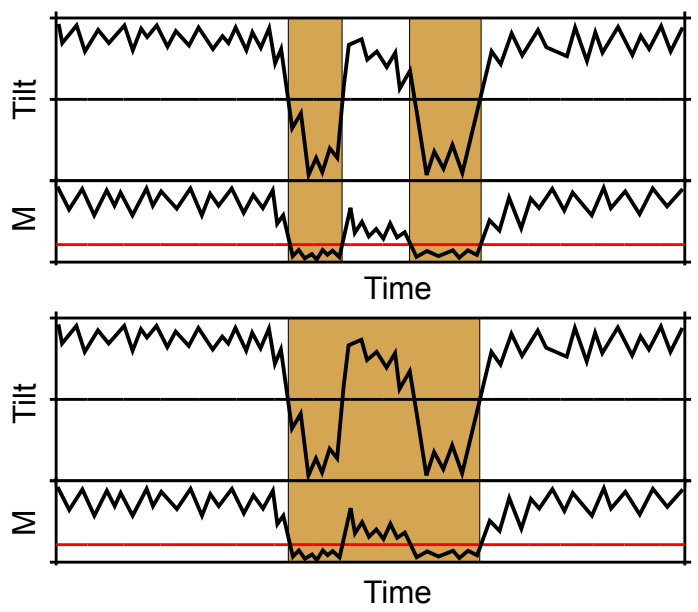

Figure 5.10: Sketches of dipole tilt (upper sub-panels) and dipole moment (lower subpanels) time series illustrating the effect of the stable period $\mathcal{T}_{S}$ on different combinations of consecutive events: successive reversals (panels a), grand excursion and reversal (panels b) and successive grand excursions (panels c). For each combination of events, the time series before and after the application of the temporal filter are shown in the upper and lower panels respectively. The horizontal red line denotes the critical dipole moment $M_{C}$ used to define the event durations. 
this problem to occur decreases with $\mathcal{T}_{S}$ so that $\mathrm{P} 2$ is more severe for combination of low $\mathcal{M}_{C}$ and short $\mathcal{T}_{S}$.

Problem P3 concerns the other extreme where we falsely regard a 'proper' SPI as part of an ongoing event because $\mathcal{M}_{C}$ is chosen too large and/or $\mathcal{T}_{S}$ too long. Since two events are melted to form a single long one, the statistics of event durations can be severely modified. P3 particularly concerns combinations of large $\mathcal{M}_{C}$ and long $\mathcal{T}_{S}$. All three problems more drastically impact the duration of the shorter events than of the longer SPIs.

A further problem illustrated in Figure 5.11 is P4. When $\mathcal{T}_{S}$ is unreasonably large, SPIs shorter than $\mathcal{T}_{S}$ are classified as grand excursions regardless of $\mathcal{M}_{C}$. The SPIs to the left and right of this disregarded epoch are melted to form a longer SPI. The disregarded epoch, on the other hand, will also affect the short end of the distribution of the excursion durations. In conclusion, intermediate $\mathcal{M}_{C}$ and small to intermediate $\mathcal{T}_{S}$ lead to the most reliable definition of reversals and grand excursions and we will further discuss this issue below.

The characterization of the correlations between low dipole moments and transitional
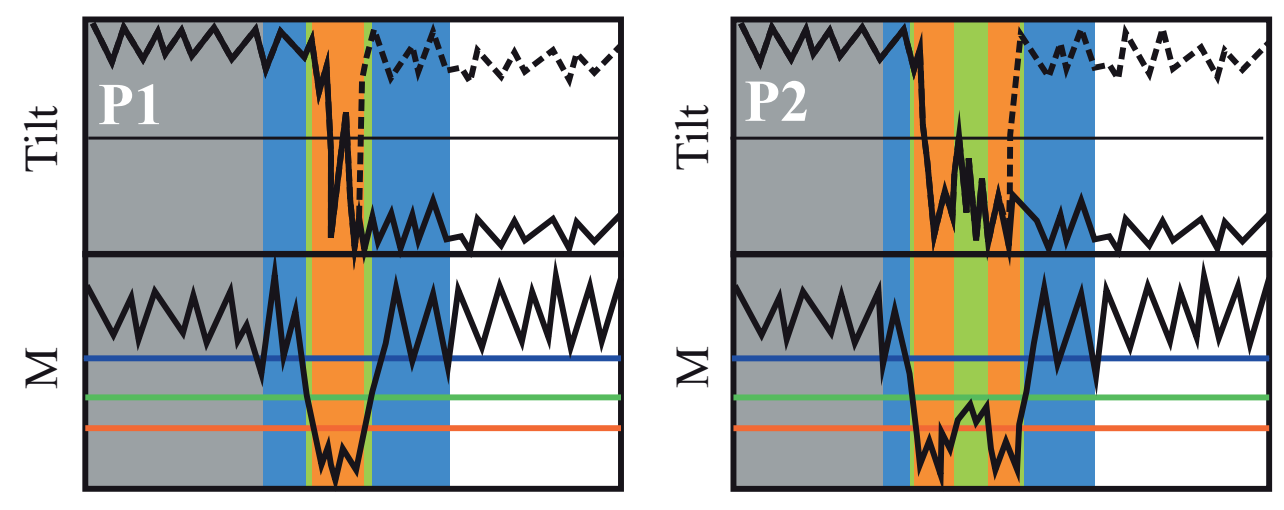

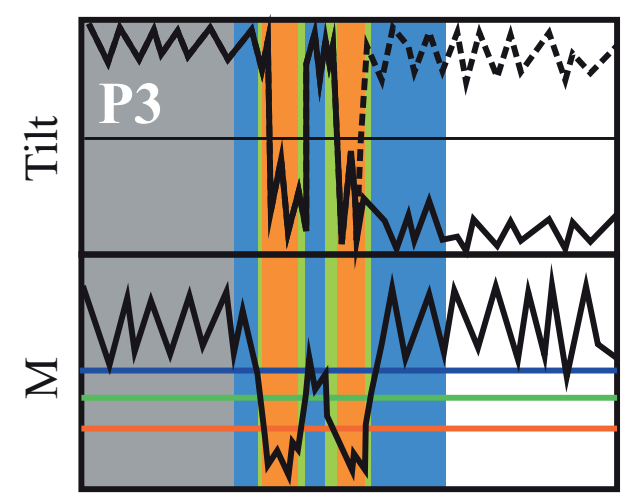

Time

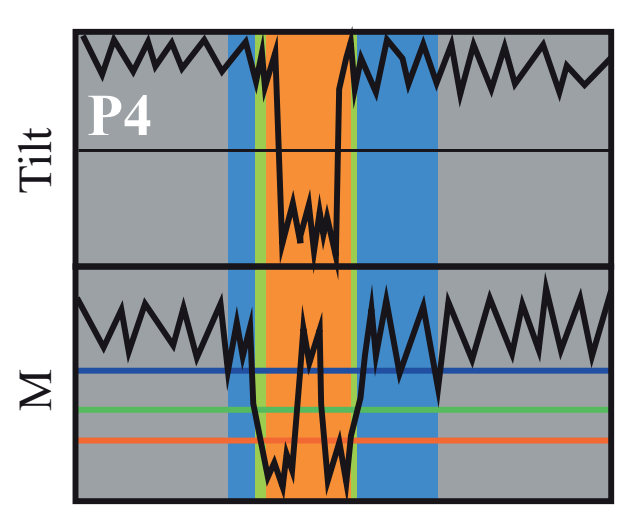

Time

Figure 5.11: Artist rendering of four different problems encountered in defining reversals and grand excursions. Top sub-panels show the dipole tilt while bottom sub-panels show the dipole moment. The solid (dashed) dipole tilt curve in each panel depicts a reversal (grand excursion). Blue, green and orange illustrate the effect of different critical dipole moments $\mathcal{M}_{C}$ (horizontal lines) on the estimate of event durations (colored backgrounds). See the main text for more explanations. 


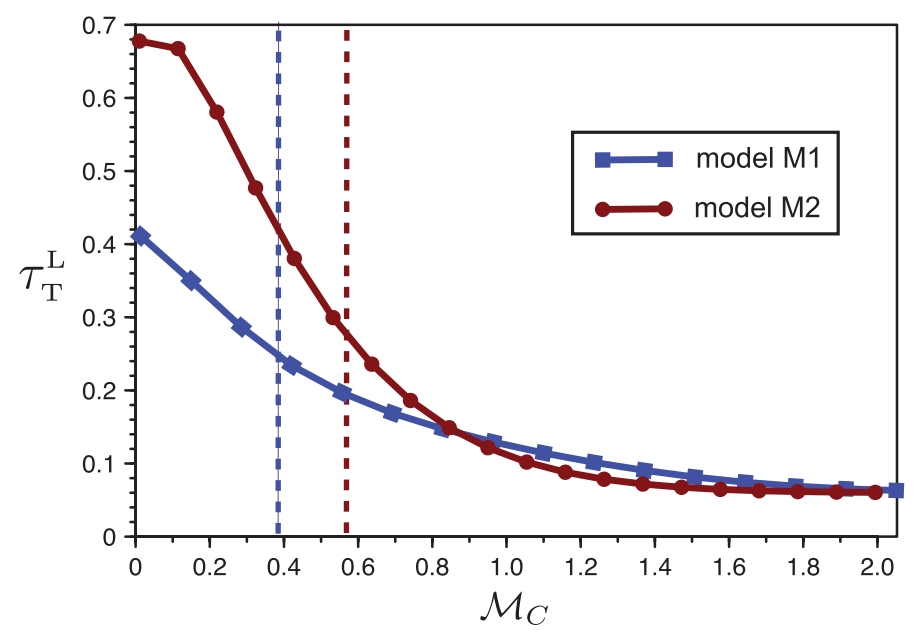

Figure 5.12: Relative time of transitional tilts during periods of low dipole field strength $\tau_{\mathrm{T}}^{\mathrm{L}}$ as function of the dipole moment threshold $\mathcal{M}_{C}$ for the dynamo models M1 and M2. Tilts are defined as transitional if the magnetic pole is further away than $45^{\circ}$ from the closest geographic pole. Vertical dashed lines mark one standard deviation below the respective time-averaged dipole moment value.

dipole tilts serves to better constrain the critical dipole moments $\mathcal{M}_{C}$ which provide reliable event definitions. Figure 5.12 shows the likelihood for transitional tilts during weak field epochs, calculated as the fraction of time spent with dipole tilts $45^{\circ} \leq \Theta \leq 135^{\circ}$ during periods with dipole moments lower than $\mathcal{M}_{C}$ and indicated as $\tau_{\mathrm{T}}^{\mathrm{L}}$, for the two dynamo models analyzed here. This measure gradually decreases with $\mathcal{M}_{C}$ in both dynamo models and slowly approaches the relative transitional times $\tau_{\mathrm{T}}$ (Table 5.2) as expected. The maximum values of $\tau_{\mathrm{T}}^{\mathrm{L}}$ are about $41 \%$ and $68 \%$ for M1 and M2 respectively. Periods of weak dipole field, therefore, do not always correlate with significant magnetic pole swings. The significantly lower value of $\tau_{\mathrm{T}}^{\mathrm{L}}$ obtained for M1 reflects the peculiar dynamics of this model at low field intensities (Section 5.2). Critical dipole moment values of $\mathcal{M}_{C}=1-\sigma_{M} / \bar{M}$, where $\sigma_{M}$ is the dipole moment standard deviation, roughly coincide with the largest variations of $\tau_{\mathrm{T}}^{\mathrm{L}}$ in Figure 5.12. These value seems to provide a reasonable estimate for the amplitude of the dipole moment variations required to enter a transitional phase in each model.

However, since no clear cut-off in the likelihood for transitional times exists, it is impossible to fix a single value for the critical dipole moment $\mathcal{M}_{C}$ which properly defines reversals and excursions. For this reason, we perform a statistical analysis varying systematically $\mathcal{M}_{C}$ and the stable period $\mathcal{T}_{S}$. This study has not been performed before in the literature and is of fundamental importance to guarantee the robustness of the statistical results.

\subsubsection{Selection of grand excursions}

As already pointed out before, numerical simulations suggest that large drops in dipole strength are required to regard excursions as global events (Wicht 2005, Wicht et al. 2009). A reasonable definition of grand excursions therefore considers these events as character- 
ized by dipole moment dips similar to reversals. In order to choose a reasonable value for the critical dipole moment $\mathcal{M}_{C}^{\mathcal{E}}$ in the dynamo model M2, we calculated the mean dipole moment minimum reached during reversals and grand excursions $\left\langle\mathcal{M}_{\min }\right\rangle$ when the dipole moment threshold $\mathcal{M}_{C}=\mathcal{M}_{C}^{\varepsilon}$ is varied (Figure 5.13). This measure rapidly increases below $\mathcal{M}_{C} \simeq 0.2$ because events with larger dipole moment minima are progressively taken into account, and tends to saturate for larger $\mathcal{M}_{C}$ values. Note, however, that $\left\langle\mathcal{M}_{\text {min }}\right\rangle$ slightly decreases for $\mathcal{M}_{C} \gtrsim 0.2$ due to problem P3 of Figure 5.11. Too large critical dipole moments $\mathcal{M}_{C}$ have the drawback of merging consecutive events even though the dipole moment significantly recover during the in between period. The discarded events, characterized by larger dipole moment minima, cause the observed decrease in $\left\langle\mathcal{M}_{\min }\right\rangle$. In conclusion, a threshold of $\mathcal{M}_{C}^{\mathcal{E}}=0.2$ seems to properly define grand excursions and this value will be kept fixed in the following statistical analysis.

It is remarkable to notice that the dipole moment reaches, on average, minimum values of about $5-6 \%$ of $\bar{M}$ during reversals and grand excursions. Standard deviations in Figure 5.13 give an idea of the dipole moment variability around these values and do not significantly differ between the two types of events.

As an example, Figure 5.14 shows the histogram of the dipole moment minima $\mathcal{M}_{\text {min }}$ during reversals and grand excursions for the combination $\mathcal{M}_{C}=0.3, \mathcal{T}_{S}=0.2$ and $\mathcal{M}_{C}^{\mathcal{E}}=0.2$. The distributions are very similar as expected from the above analysis. The most likely dipole moment minimum reached during reversals and grand excursions is around $3 \%$ of the time-averaged dipole moment $\bar{M}$.

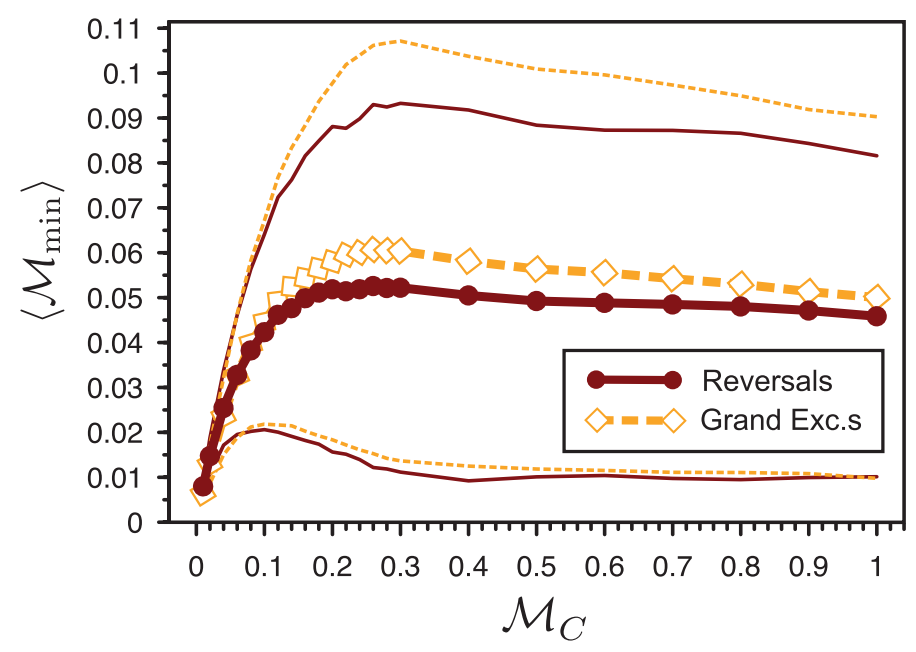

Figure 5.13: Mean dipole moment minima reached $\left\langle\mathcal{M}_{\min }\right\rangle$ during reversals and grand excursions (in units of the time-averaged dipole moment $\bar{M}$ ) as function of the critical dipole moment $\mathcal{M}_{C}$ for the dynamo model M2. The thin curves indicate the $1 \sigma$-intervals of the respective estimates. The stable period has been fixed to $\mathcal{T}_{S}=0.1$. 


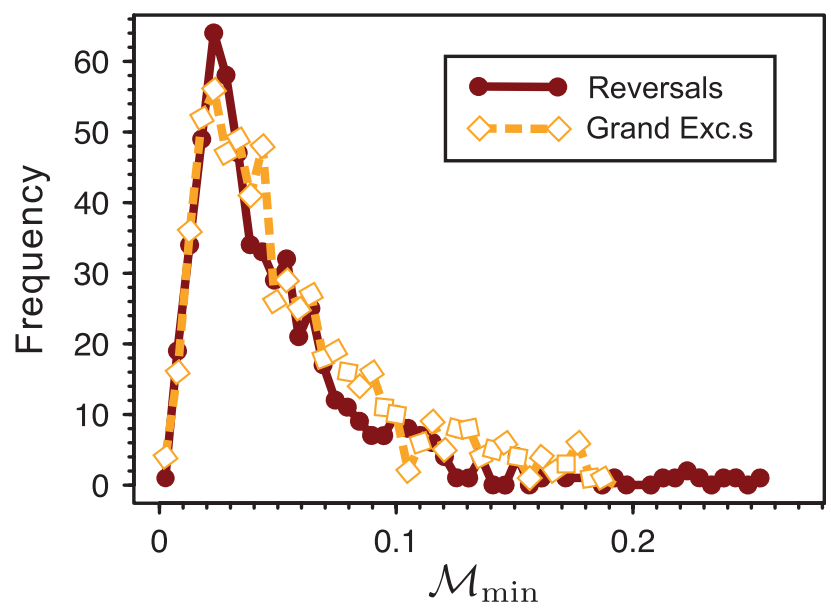

Figure 5.14: Histograms of the dipole moment minima reached during reversals and grand excursions $\mathcal{M}_{\text {min }}$ (in units of the time-averaged dipole moment $\bar{M}$ ) for the dynamo model M2 obtained using the threshold combination $\mathcal{M}_{C}=0.3, \mathcal{M}_{C}^{\mathcal{E}}=0.2$ and $\mathcal{T}_{S}=0.2$.

\subsection{Distribution of the durations of stable polarity inter- vals, reversals and excursions}

Based on the methods described in Chapter 3, we studied the statistical properties of reversals and grand excursions of the dynamo model M2. The analysis focuses on probability distributions previously used to describe the occurrence of geomagnetic reversals (see, e.g., Cox 1968, McFadden and Merrill 1993, Jonkers 2003, Ryan and Sarson 2007, Shcherbakov and Fabian 2012). In order to explore the dependence of the statistical results on our definition of reversals and excursions, the dipole moment threshold $\mathcal{M}_{C}$ and the stable period $\mathcal{T}_{S}$ have been varied systematically. In particular, the dipole moment threshold has been varied from $\mathcal{M}_{C}=0.1$ to $\mathcal{M}_{C}=0.8$ in steps of $\mathcal{M}_{C} / 10$ and the stable period from $\mathcal{T}_{S}=0.1$ to $\mathcal{T}_{S}=1$ in steps of $\mathcal{T}_{S} / 10$. We recall here that $\mathcal{M}_{C}$ is normalized with the time averaged dipole moment $\bar{M}$ (see Table 5.2) and $\mathcal{T}_{S}$ is given in units of the magnetic diffusion time $\tau_{\eta}$. Since model M2 represents more closely the Earth's behavior compared to M1 (see Section 5.1), the following statistical analysis concentrates on the former model.

Different reversal and excursion sequences are obtained for each parameter combination $\left(\mathcal{M}_{C}, \mathcal{T}_{S}\right)$. In the following, $\mathcal{R}$ indicates the set of reversal durations obtained for a specific combination of these parameters, namely

$$
\mathcal{R}=\left\{\Delta t_{i}^{R}\right\}_{i=1}^{N_{R}}
$$

In a similar way, we refer to

$$
\mathcal{E}=\left\{\Delta t_{i}^{E}\right\}_{i=1}^{N_{E}}
$$

to as the set of grand excursion durations, and to

$$
\mathcal{P}=\left\{\Delta t_{i}^{P}\right\}_{i=1}^{N_{P}}
$$


to as the set of stable polarity interval (SPI) durations for a given parameter combination $\left(\mathcal{M}_{C}, \mathcal{T}_{S}\right)$. The event durations $\Delta t_{i}^{R}, \Delta t_{i}^{E}$ and $\Delta t_{i}^{P}$ have been calculated using the algorithm described in Section 5.3.

\subsubsection{Stable polarity intervals and occurrence of reversals}

A suite of probability distributions (exponential, gamma, Weibull, log-normal and loglogistic as described in Section 3.4.1) are fitted to the SPI durations $\mathcal{P}$ for each combination of $\mathcal{M}_{C}$ and $\mathcal{T}_{S}$ explored. Since SPIs cannot be shorter than $\mathcal{T}_{S}$ by construction (Section 5.3), left truncated distribution functions are used. Distribution parameters are estimated using the maximum likelihood (ML) method described in Section 3.5. We assess the goodness-of-fit computing the Kolmogorov-Smirnov (KS) p-values $\alpha_{K S}$ and the Anderson-Darling (AD) p-values $\alpha_{A D}$ (Section 3.6). Since a significance level of $5 \%$ is assumed, This means that the distribution under testing (null-hypothesis) will be rejected if neither $\alpha_{K S}$ nor $\alpha_{A D}$ exceed 0.05 .

Figure 5.15] summarizes the distribution fits of the SPI durations $\mathcal{P}$ obtained for the combinations $\left(\mathcal{M}_{C}, \mathcal{T}_{S}\right)$ explored. The distribution functions are marked with different

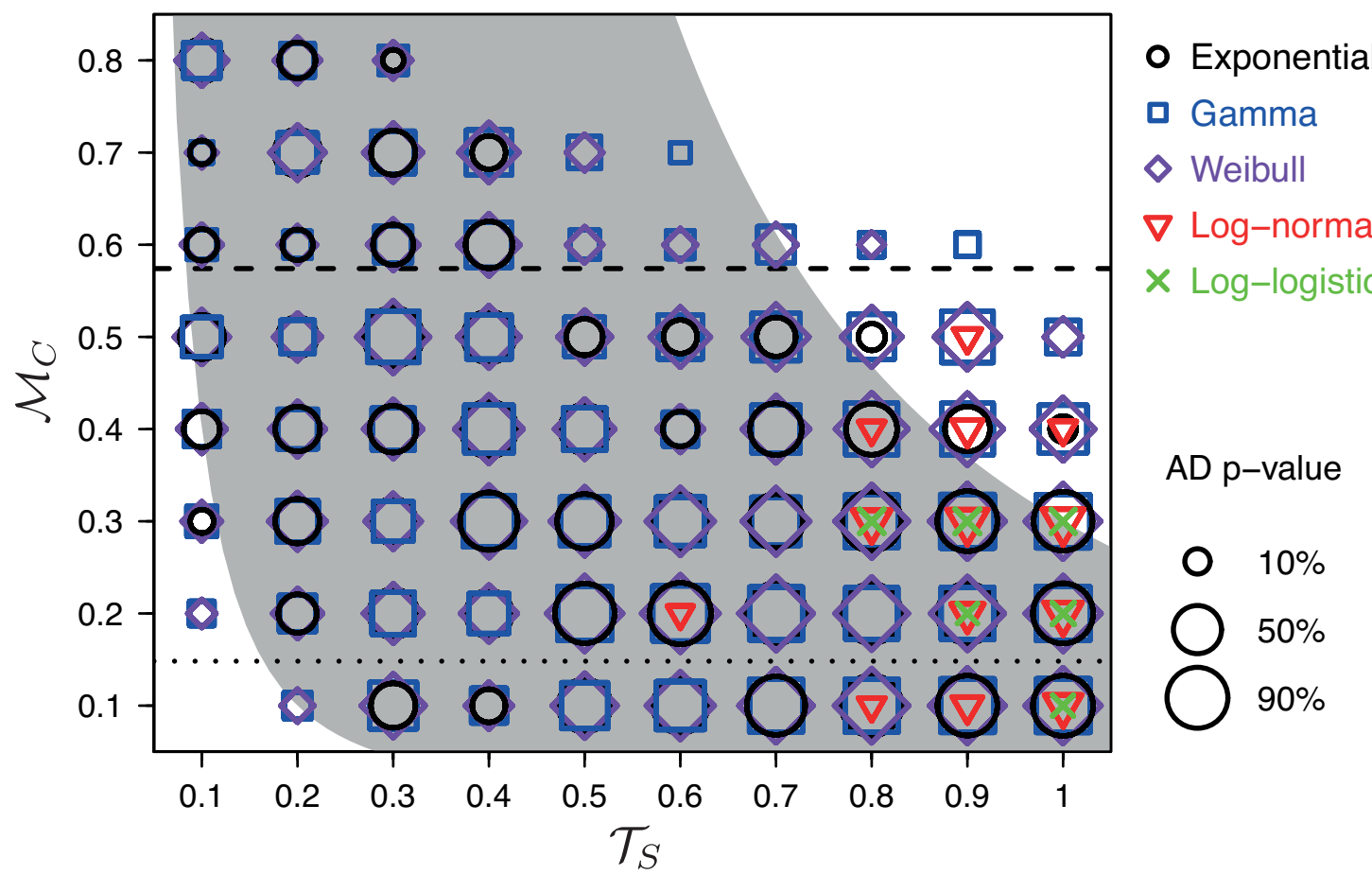

Figure 5.15: Anderson-Darling (AD) p-values of the distribution fits of the stable polarity interval durations $\mathcal{P}$ obtained for the threshold values $\mathcal{M}_{C}$ and $\mathcal{T}_{S}$ explored. The tested distribution functions are marked with different colored symbols (top right legend). Symbol sizes are scaled with the associated AD p-value (bottom right legend). Empty spaces occur where all the distribution functions can be rejected at the 5\% significance level. The dashed (dotted) horizontal line marks one (two) standard deviation(s) below the timeaveraged dipole moment value. The gray background highlights parameter combinations expected to give reliable event definitions (see the main text for details). 
colored symbols and the symbol sizes are scaled with the corresponding AD p-value. Exponential, gamma and Weibull distributions cannot be rejected at the $95 \%$ confidence level for most points of the parameter space. Moreover, practically identical p-values are obtained for all three distributions. The exponential can be rejected only at particularly small or large combinations of $\mathcal{M}_{C}$ and $\mathcal{T}_{S}$. Note furthermore that none of the distribution functions fits the data for high $\mathcal{M}_{C}$ and $\mathcal{T}_{S}$ values.

Closer inspection reveals that certain parameter combinations lead to unreasonable definitions of reversals and excursions due to the problems discussed in Section 5.3. Figure 5.16 shows the dependence of the mean SPI duration on the product $\mathcal{M}_{C} \mathcal{T}_{S}^{2}$. This empirical combination provides an acceptable scaling of the SPI durations. Black dots mark threshold combinations where the exponential distribution fits cannot be rejected at the given significance level, while blue squares represent cases where the exponential is rejected but gamma and/or Weibull are not. Red triangles show significant log-normal fits and finally crosses indicate parameter combinations where none of the distribution functions is able to describe the data. The mean SPI duration is less sensitive to the parameter choice for intermediate values of $\mathcal{M}_{C} \mathcal{T}_{S}^{2}$ which therefore seem to offer more reasonable definitions of reversals and excursions. If $\mathcal{M}_{C}$ is unreasonably low and rapid dipole moment fluctuations around $\mathcal{M}_{C}$ are not compensated by sufficiently high $\mathcal{T}_{S}$ values, short SPIs enter the data sets. This effect, identified as problem P2 in Section 5.3, leads to the more rapid increase in the mean SPI duration for small $\mathcal{M}_{C} \mathcal{T}_{S}^{2}$ values illustrated in Figure 5.16 and to a somewhat distorted statistics.

Large $\mathcal{M}_{C}$ and $\mathcal{T}_{S}$ values also show a fast increase in the mean SPI duration. The

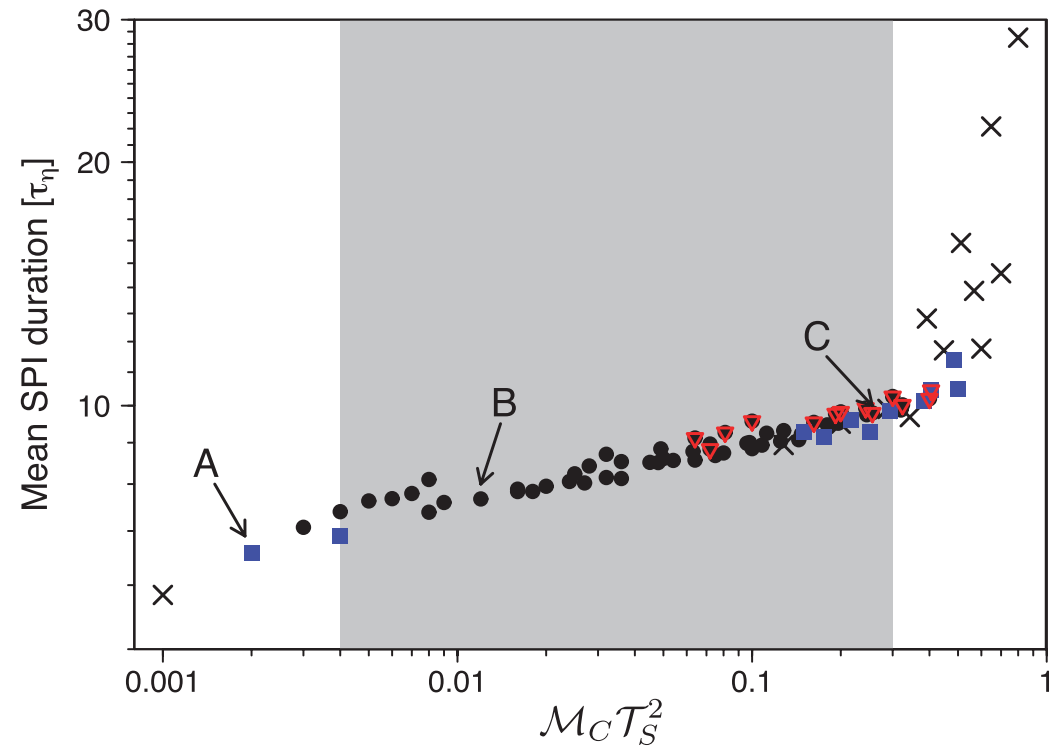

Figure 5.16: Mean stable polarity interval (SPI) duration as function of the threshold product $\mathcal{M}_{C} \mathcal{T}_{S}^{2}$. Black dots mark threshold combinations where the exponential distribution fits cannot be rejected with $95 \%$ confidence. Blue squares (red triangles) mark remaining gamma and/or Weibull (log-normal) fits. Crosses indicate where all the distribution fits are rejected. The gray background highlights the region with the less sensitive choice of threshold values. Capital letters denote the threshold combinations selected for a detailed analysis (see the main text). 
filtering introduced by the stable period $\mathcal{T}_{S}$ becomes dominant in this region of the parameter space where the shorter SPIs are likely to be combined with the adjacent polarities, thus leading to unreasonably long event durations (problem P4 in Section 5.3). As a consequence, none of the selected models is able to describe the data.

The region with the less sensitive choice of $\mathcal{M}_{C}$ and $\mathcal{T}_{S}$ give acceptable event definitions and it is highlighted with a gray background in Figures 5.15 and 5.16. The exponential distribution dominates this part of the parameter space. Gamma and Weibull provide a good description of the reliable $\mathcal{P}$ sequences towards higher values of both $\mathcal{M}_{C}$ and $\mathcal{T}_{S}$ (cf. Figure 5.15). Furthermore, note that the log-normal and log-logistic distributions tend to cluster at particularly high $\mathcal{T}_{S}$ values, thus around the boundary with the unreasonably long SPIs. As we will demonstrate below, these heavy tailed distribution functions naturally account for longer event durations.

Three parameter combinations, representative of the different event definitions depicted above, are indicated in Figure 5.16, a combination of low threshold values with $\mathcal{M}_{C}=0.2$ and $\mathcal{T}_{S}=0.1$ (case A), an intermediate choice of $\mathcal{M}_{C}=0.3$ and $\mathcal{T}_{S}=0.2$ (case B) and the extreme combination $\mathcal{M}_{C}=0.4$ and $\mathcal{T}_{S}=0.8$ (case C). Case A has the lowest mean SPI duration, while case B belongs to the region of reasonable event definitions (cf. Figure 5.2). Finally, case $\mathrm{C}$ belongs to the boundary with the biased event counting and presents a log-normal fit to the data.

Figure 5.17 shows the probability density functions (PDFs) and the cumulative distribution functions (CDFs) of the data and the respective fits for the three threshold combinations selected. The estimated distribution parameters are listed in Table 5.4 and the associated KS and AD p-values are given in Table 5.5.

Gamma and Weibull fit the SPI durations obtained for the threshold combination A at the given significance level and their profiles are practically indistinguishable (Figure 5.17, panels $\mathrm{a}$ and $\mathrm{b}$ ). The estimated gamma and Weibull shape parameters are $k=0.77 \pm 0.05$ and $s=0.88 \pm 0.03$ respectively, thus resulting in monotonically decreasing PDFs. As already mentioned above, an excess of short SPI durations is expected using too low $\mathcal{M}_{C}$ and $\mathcal{T}_{S}$ values (cf. problem P2 of Section 5.3).

The exponential distribution, together with gamma and Weibull, can model the SPI durations $\mathcal{P}$ obtained for the intermediate threshold combination B. As expected, the estimated gamma and Weibull shape parameters are close to one where the two distributions coincide with the exponential (see Section 3.4.1). The estimated gamma shape parameter $k$ is $0.91 \pm 0.07$, while the Weibull shape $s$ is one in the limits of statistical errors $(s=0.96 \pm 0.04)$. Rate parameters $\lambda$ of the exponential, gamma and Weibull distributions are also equal in the limits of statistical errors as expected (cf. Table 5.4). However, despite the evidences in favor of the exponential model, we cannot formally reject the hypothesis of either gamma or Weibull distributed stable polarity intervals for case B.

The longest stable polarity epoch in the numerical simulation lies significantly off any distribution profile (Figure 5.17 panel c). Its duration, which amounts to more than 60 magnetic diffusion times, is also remarkably longer than the second longest polarity interval. Such event is punctuated only by few brief grand excursions. However, as will be shown in Chapter 6, this extreme event cannot be regarded as a superchron.

Case $\mathrm{C}$ deals with the extreme situation in which $\mathcal{T}_{S}$ is so large that the stable polarity intervals defined certainly contain unreasonably long events. Compared to the the threshold combinations A and B, the histogram of SPI durations for case C presents a 


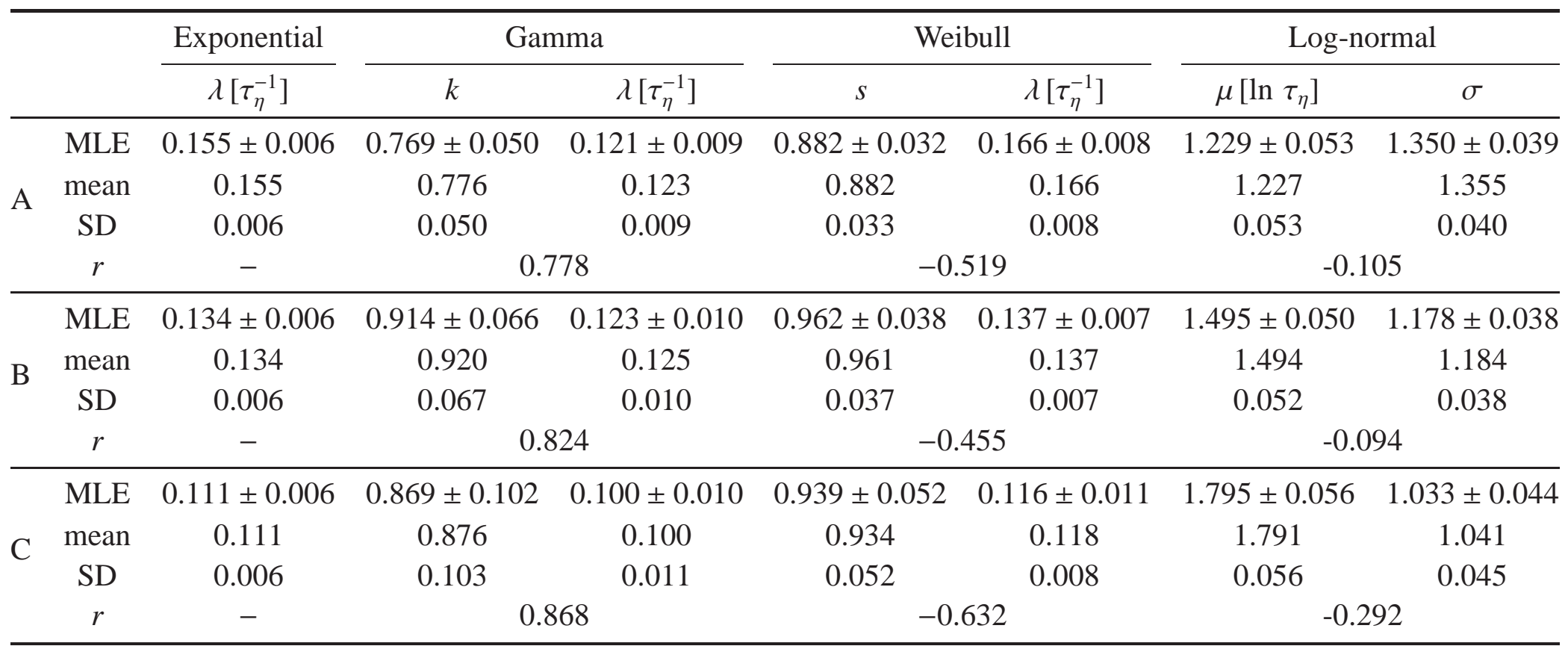

Table 5.4: Frequentist and Bayesian parameter inference of exponential, gamma, Weibull and log-normal distribution functions for the stable polarity interval durations $\mathcal{P}$ obtained for the combinations $\mathcal{M}_{C}=0.2$ and $\mathcal{T}_{S}=0.1(\mathrm{~A}), \mathcal{M}_{C}=0.3$ and $\mathcal{T}_{S}=0.2(\mathrm{~B}), \mathcal{M}_{C}=0.4$ and $\mathcal{T}_{S}=0.8(\mathrm{C})$. Maximum likelihood estimates (MLE) of the distribution parameters are given in the first row of each threshold combination (errors refer to the $68 \%$ confidence limits of the relative parameter estimate). Rows from 2 to 4 of each threshold combination list mean, standard deviation (SD) and the Pearson correlation coefficient $r$ of the posterior density of the different distribution parameters. 

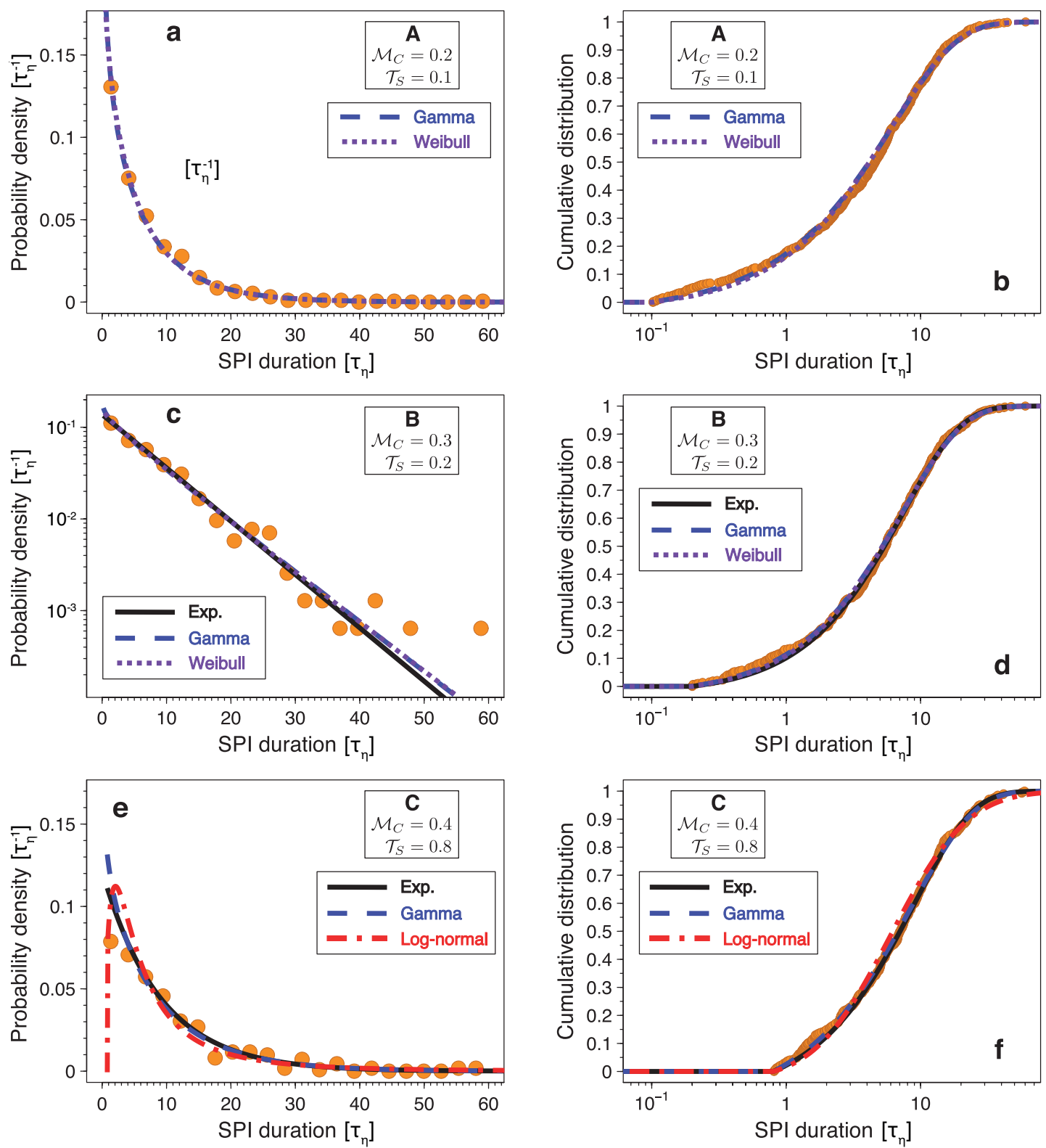

Figure 5.17: Probability density functions (left column) and cumulative distributions (right column) of the stable polarity interval (SPI) durations obtained for $\mathcal{M}_{C}=0.2$ and $\mathcal{T}_{S}=0.1$ (case A, panels a and b), $\mathcal{M}_{C}=0.3$ and $\mathcal{T}_{S}=0.2$ (case B, panels $\mathrm{c}$ and $\mathrm{d}$ ), $\mathcal{M}_{C}=0.4$ and $\mathcal{T}_{S}=0.8$ (case $\mathrm{C}$, panels e and $\mathrm{f}$ ). The colored curves represent the distribution fits passed at the $95 \%$ confidence level (see the legend insets).

diminished probability at its low end and several additional events with longer durations (Figure 5.17 panel e). The temporal filter $\mathcal{T}_{S}$ does not simply discard SPIs with a duration lower than $0.8 \tau_{\eta}$, but also merges adjacent events into longer stable polarity epochs thus biasing the statistics (problem P4 of Section 5.3). Exponential, gamma and Weibull rate parameters $\lambda$ become consequently lower compared to cases A and B (Table 5.4). Gamma and Weibull shape parameters are somewhat closer to case B but larger than the estimated 
values in case $\mathrm{A}$ as expected.

The log-normal distribution also provides a reasonable fit to the data and, strictly speaking, it cannot be rejected at the 5\% significance level (cf. Table 5.5). Its probability density is not monotonically decreasing as for the former two distributions (Figure 5.17, panel e). The log-normal may fit better the SPI shorter than about $20 \tau_{\eta}$, but at the price of a heavier tail overestimating the actual contribution from the data (Figure 5.17 panel $\mathrm{f}$ ). As a final remark note that two of the longer events in case $C$ have an artificially enhanced duration compared to cases A and B and almost reach the longest SPI. These examples reveal the severe effect of the filtering algorithm on the SPI durations and the preferable moderate choices for the $\mathcal{M}_{C}$ and $\mathcal{T}_{S}$ values.

The frequentist analysis performed here has shown that none of the exponential, gamma and Weibull hypotheses can be rejected at the 5\% significance level when describing the stable polarity interval durations in model M2. We therefore resort to a Bayesian approach in order to quantify the relative likelihood between the selected models (Section 3.7).

Figure 5.18 summarizes the posterior probabilities (3.103) of the distribution functions for the SPI durations $\mathcal{P}$ obtained for the combinations of $\mathcal{M}_{C}$ and $\mathcal{T}_{S}$ explored. Only distribution functions which passed the goodness-of-fit tests are shown (cf. Figure 5.15). Colored symbols denote different distribution functions and the symbol sizes are scaled with the corresponding posterior probability. For each parameter combination, the relative sizes of the symbols correspond to the respective posterior odds ratios as given by Equation (3.106). Since no preference to any particular distribution is given a priori, the prior model probabilities in (3.106) are assumed to be equal.

Prior probabilities of the distribution parameters are assumed to be uniform (see Section 3.7.2). In particular, the exponential rate parameter $\lambda$ has been chosen to range from the lowest possible rate $\lambda_{\min }=1 / \tau$, where $\tau$ is the total simulation time (Table 5.2), to a maximum value of $\lambda_{\max }=1 / \mathcal{T}_{S}$. Priors for gamma and Weibull rate parameters range

\begin{tabular}{cccccc}
\hline & & Exponential & Gamma & Weibull & Log-normal \\
\hline & $\alpha_{K S}$ & 6.1 & 16.9 & 22.6 & 0 \\
$\mathrm{~A}$ & $\alpha_{A D}$ & 0.5 & 12.5 & 7.6 & 0 \\
& $\mathrm{P}$ & 0.3 & 94.5 & 5.2 & 0 \\
\hline & $\alpha_{K S}$ & 56.4 & 43.4 & 52.3 & 0.09 \\
$\mathrm{~B}$ & $\alpha_{A D}$ & 41.1 & 46.2 & 45.1 & 0.03 \\
& $\mathrm{P}$ & 90.2 & 6.7 & 3.1 & 0 \\
\hline & $\alpha_{K S}$ & 53.2 & 81.3 & 82.1 & 6.2 \\
$\mathrm{C}$ & $\alpha_{A D}$ & 57.9 & 81.9 & 80.2 & 5.3 \\
& $\mathrm{P}$ & 85.7 & 9.3 & 5.0 & 0 \\
\hline
\end{tabular}

Table 5.5: Kolmogorov-Smirnov p-values $\alpha_{K S}$, Anderson-Darling p-values $\alpha_{A D}$ and Bayesian posterior model probabilities $\mathrm{P}$ for different distribution functions relative to the stable polarity interval durations $\mathcal{P}$ obtained for the threshold combinations $\mathcal{M}_{C}=0.2$ and $\mathcal{T}_{S}=0.1(\mathrm{~A}), \mathcal{M}_{C}=0.3$ and $\mathcal{T}_{S}=0.2(\mathrm{~B}), \mathcal{M}_{C}=0.4$ and $\mathcal{T}_{S}=0.8(\mathrm{C})$. Values are given in percentages. 


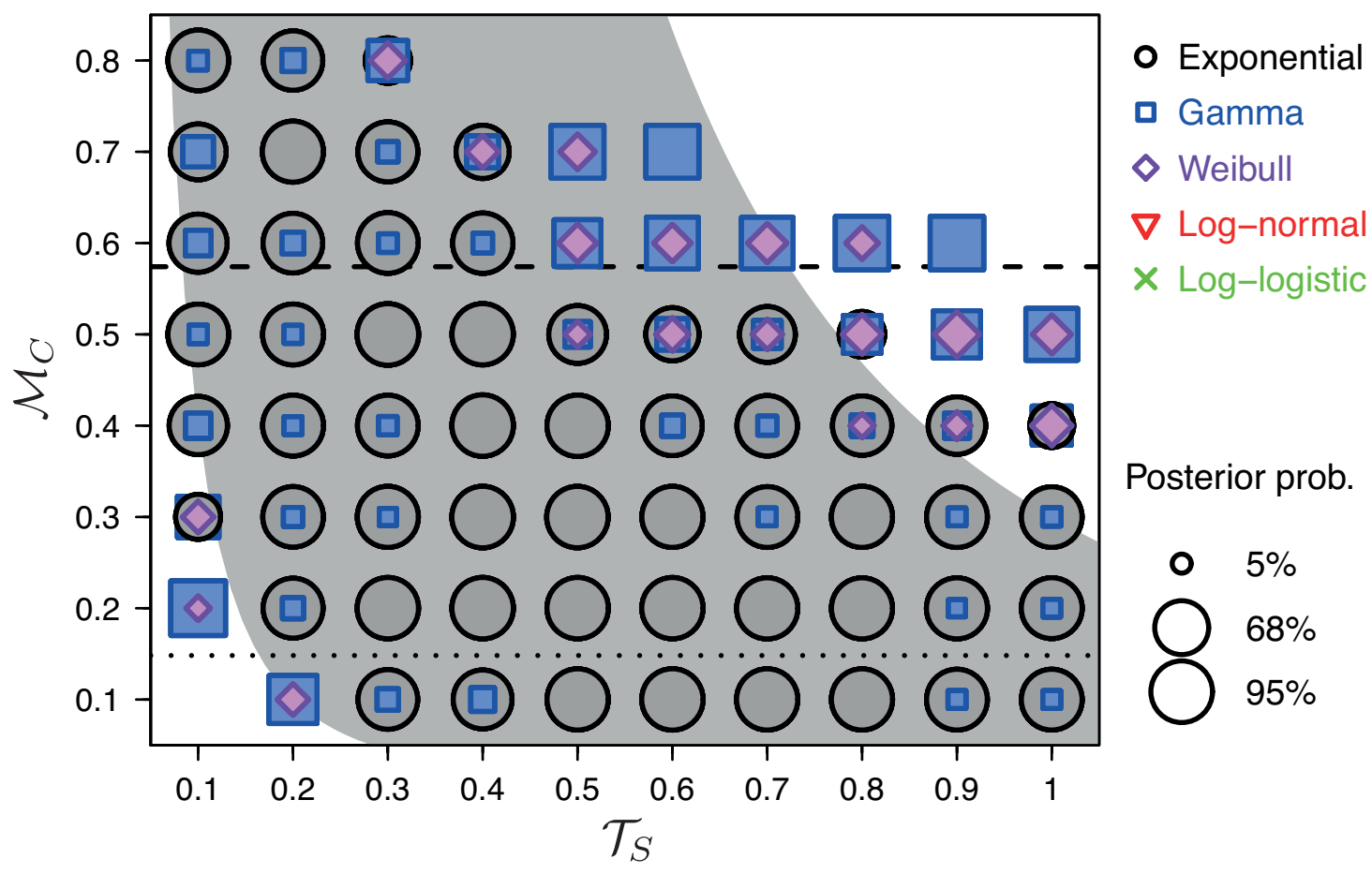

Figure 5.18: Bayesian posterior probabilities of the distribution functions (3.103) for the stable polarity interval durations $\mathcal{P}$ obtained for the threshold values $\mathcal{M}_{C}$ and $\mathcal{T}_{S}$ explored. The tested distribution functions are marked with different colored symbols (top right legend). Symbol sizes are scaled with the associated posterior probability (bottom right legend). Empty spaces occur where all the distribution functions have been rejected at the 5\% significance level (cf. Figure 5.15). The dashed (dotted) horizontal line marks one (two) standard deviation(s) below the time-averaged dipole moment value. The gray background highlights parameter combinations expected to give reliable event definitions.

in the same interval. Shape parameters are more difficult to constrain a priori. Since highly skewed distribution functions are not expected, we restricted the uniform priors of the gamma and Weibull shape parameters within the interval $(0,5]$. Priors for the lognormal mean $\mu$ and standard deviation $\sigma$ are assumed to be uniform between $[-10,10]$ and $(0,5]$ respectively. Priors for the log-logistic scale parameter $\theta$ and the shape parameter $\alpha$ vary in $(0,20]$ and $(0,10]$ respectively. Note that we defined similar prior ranges for the two-parameters distribution functions. This choice minimizes the penalization factor introduced by larger prior uncertainties (see Section 3.7.4).

For most of the $\mathcal{M}_{C}$ and $\mathcal{T}_{S}$ combinations explored, the exponential distribution is clearly preferred with posterior probabilities above 95\% (Figure 5.18). The gamma distribution appears as a second contender for certain threshold values. Strong evidences in favor of the gamma model mainly occur where the event definitions are not reliable, that is at particularly small or large combinations of $\mathcal{M}_{C}$ and $\mathcal{T}_{S}$.

Figure 5.19 shows the Bayesian posterior densities of the exponential rate parameter $\mathrm{P}\left(\lambda \mid \mathcal{P}\right.$, Exp) calculated from Equation (3.99) for the threshold combinations B $\left(\mathcal{M}_{C}=\right.$ $\left.0.3, \mathcal{T}_{S}=0.2\right)$ and $\mathrm{C}\left(\mathcal{M}_{C}=0.4, \mathcal{T}_{S}=0.8\right)$ selected above. The most likely values of the rate $\lambda$ are $0.134 \tau_{\eta}^{-1}$ and $0.111 \tau_{\eta}^{-1}$ for $\mathrm{B}$ and $\mathrm{C}$ respectively and agree with the sample 

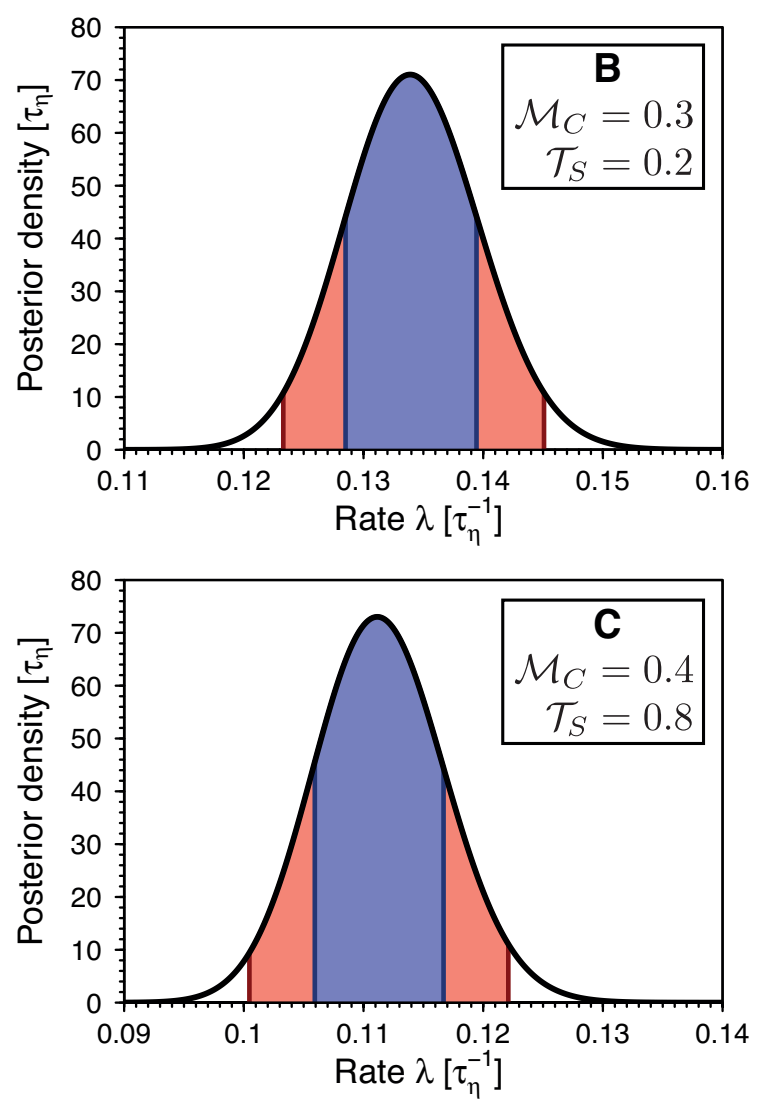

Figure 5.19: Bayesian posterior densities of the exponential rate parameter $\lambda$ for the stable polarity interval durations $\mathcal{P}$ obtained for the threshold combinations $\mathrm{B}\left(\mathcal{M}_{C}=0.3\right.$ and $\mathcal{T}_{S}=0.2$, top panel $)$ and $\mathrm{C}\left(\mathcal{M}_{C}=0.4\right.$ and $\mathcal{T}_{S}=0.8$, bottom panel $) .68 \%$ and $95 \%$ highest posterior density intervals are marked in blue and red respectively.

means of the respective SPI durations as expected (cf. Figure 5.16). Due to the higher $\mathcal{M}_{C}$ and $\mathcal{T}_{S}$ values of case $\mathrm{C}$ which provide longer SPIs, the posterior distribution shifts towards lower values of $\lambda$.

Since a uniform prior has been assumed, the posterior modes coincide with the ML estimates of $\lambda$ because, as shown by Equation (3.97), the posterior distribution is proportional to the likelihood function. Moreover, the posterior densities in Figure 5.19 are practically identical to normal distributions as expected in the asymptotic limit of the likelihood function (see Section 3.5.3). The number of simulated polarity intervals is thus sufficient to guarantee the robustness of the statistical results. Drawing random samples from $\mathrm{P}(\lambda \mid \mathcal{P}$, Exp) permits to calculate its posterior mean and standard deviation. These values are reported in Table 5.4 and completely specify the posterior distributions.

Bayesian credibility intervals of $68 \%$ and $95 \%$ around the mode are marked in blue and red respectively in Figure 5.19. Bayesian inference permits to conclude that the true value of $\lambda$ lies, with $68 \%$ probability, within the intervals $[0.128,0.140] \tau_{\eta}^{-1}$ and $[0.105,0.117] \tau_{\eta}^{-1}$ for the threshold combinations B and C respectively.

Figure 5.20 shows the posterior densities of the gamma and Weibull distribution parameters, namely $\mathrm{P}(k, \lambda \mid \mathcal{P}$, Gamma) and $\mathrm{P}(s, \lambda \mid \mathcal{P}$, Weibull), for the three threshold combinations selected above. These posterior densities are very close to bivariate normal 

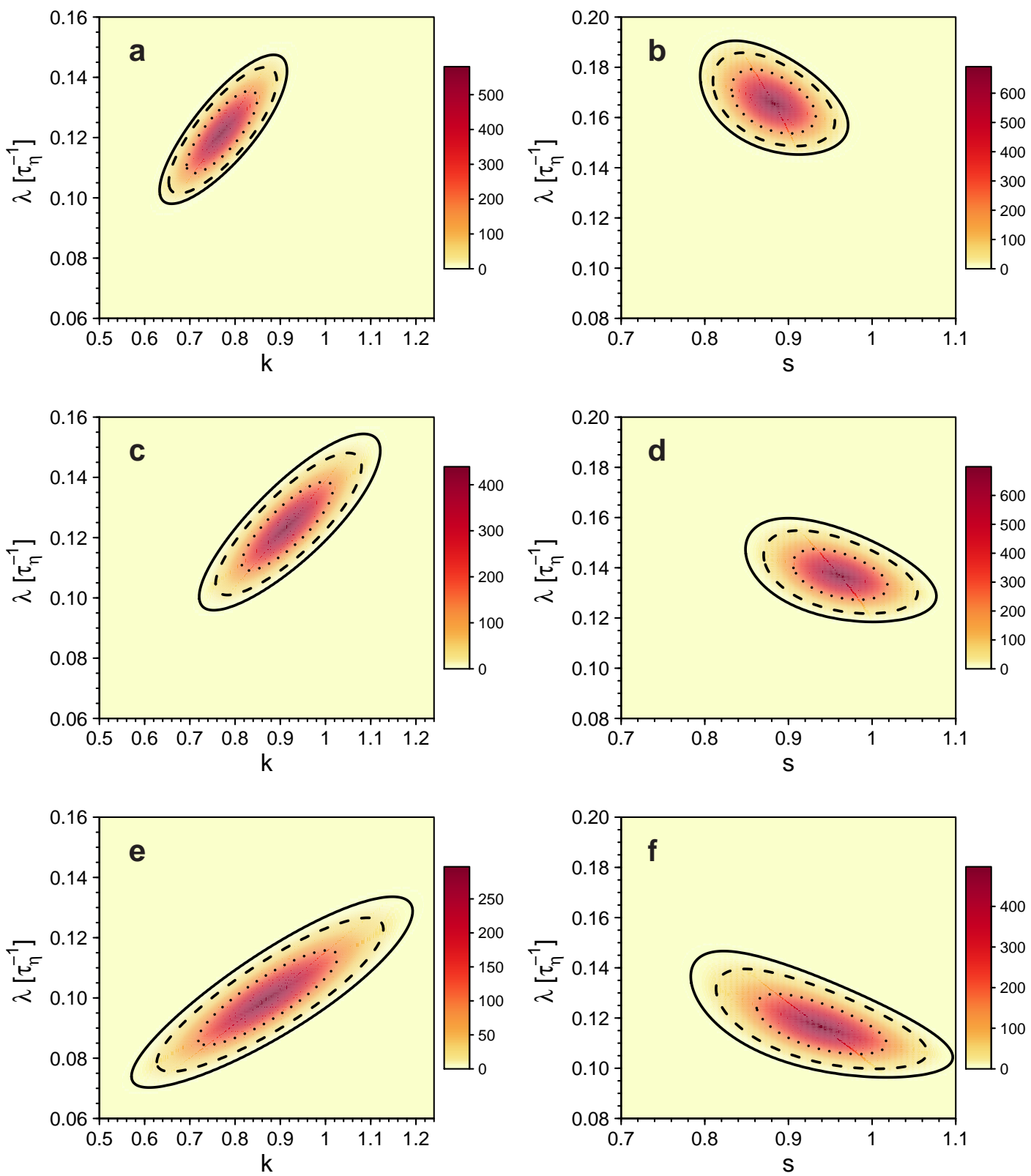

Figure 5.20: Bayesian posterior densities of gamma (left column) and Weibull (right column) distribution parameters for the stable polarity interval durations $\mathcal{P}$ obtained for the threshold combinations $\mathcal{M}_{C}=0.2$ and $\mathcal{T}_{S}=0.1$ (case A, panels a and b), $\mathcal{M}_{C}=0.3$ and $\mathcal{T}_{S}=0.2$ (case B, panels c and d), $\mathcal{M}_{C}=0.4$ and $\mathcal{T}_{S}=0.8$ (case C, panels e and f). Iso-contour lines mark the $68 \%, 95 \%$ and $99.7 \%$ highest posterior density intervals.

distributions. As already pointed out before, this indicates that the statistic is well constrained in terms of the events number. The gamma parameters $k$ and $\lambda$ are positively correlated for all the threshold combinations selected (Figure 5.20, panels a, c and e) The respective correlation coefficients are reported in Table 5.4 and reach values as high as $80 \%$.

The threshold combination A, dominated by short polarity intervals, shows a narrow gamma posterior density significantly away from $k=1$ where the distribution coincides 
with the exponential. The fact that $\mathrm{P}(k, \lambda \mid \mathcal{P}$, Gamma) is strongly peaked around its mode is a direct consequence of the large number of polarity intervals available in this case $\left(N_{P}=682\right)$. Having a model posterior probability of about $94 \%$, the evidence in favor of the gamma distribution is positive.

Larger values of $k$ become more likely when increasing $\mathcal{M}_{C}$ and $\mathcal{T}_{S}$ to the moderate threshold combination B (Figure 5.20, panel c). In particular, the 68\% highest posterior credibility interval now includes $k=1$. This is confirmed by the positive evidence in favor of the exponential distribution which presents a posterior probability of about $90 \%$, while the gamma model reaches only $7 \%$ (Table 5.5). The additional degree of freedom introduced by the second parameter of the gamma distribution is not justified by a sufficient gain in the data likelihood for this threshold combination. The posterior density of the gamma parameters relative to case $\mathrm{C}$ (Figure 5.20, panel e) covers a broad range of $k$ values and the $68 \%$ credibility interval still includes $k=1$. The correlation between the two distribution parameters also remains high $(r=0.86)$.

Posterior densities of the Weibull parameters $s$ and $\lambda$ are illustrated in the right column of Figure 5.20 for the three combinations of $\mathcal{M}_{C}$ and $\mathcal{T}_{S}$ selected. Contrary to the gamma distribution, Weibull parameters are negatively correlated with somewhat lower amplitudes (cf. Table 5.4). For $s<1$, increasing the value of $s$ results in a steeper monotonically decreasing PDF (Figure 3.7). Since the Weibull rate $\lambda$ tends to decrease in order to fit the data for larger $s$, the two distribution parameters become negatively correlated. Bivariate normal distributions only partially approximate the posterior densities $\mathrm{P}(s, \lambda \mid \mathcal{P}$, Weibull). Sample means, standard deviations and correlation coefficients of $\mathrm{P}(s, \lambda \mid \mathcal{P}$, Weibull $)$ are reported in Table 5.4 .

Contrary to the posterior densities of the gamma parameters, $\mathrm{P}(s, \lambda \mid \mathcal{P}$, Weibull) mildly shifts towards larger values of the shape parameter $s$ when going from the threshold combination A to B. The value $s=1$ is within the $68 \%$ credibility interval in the latter case, in agreement with the positive evidence in favor of the exponential model found. The posterior model probability for the Weibull distribution remains quite low for all the selected cases with values up to $5 \%$. Since the priors used for the gamma and Weibull parameters are the same, the data likelihood must account for the observed discrepancy in the posterior model probabilities. We can therefore conclude that the gamma distribution fits the SPI durations better than the Weibull for the combinations of $\mathcal{M}_{C}$ and $\mathcal{T}_{S}$ explored.

The impact of different prior choices for the distribution parameters is discussed in the following section. The results discussed above have been found to vary only mildly with the specific prior choice. This means that the posterior probabilities are dominated by the data likelihood (see Section 3.7.2) and therefore the statistical inferences obtained are robust.

In conclusion, our Bayesian analysis shows a strong evidence in favor of exponentially distributed stable polarity interval durations in dynamo model M2 over a wide range of $\mathcal{M}_{C}$ and $\mathcal{T}_{S}$ combinations. Statistically significant deviations from the exponential distribution in favor of a gamma model have been found only at too small and too large threshold values where the event definitions are less reliable. Though not rigorously tested, it seems reasonable to assume that reversals are independent events in our numerical simulations. A Poisson process (Section 3.4.2) therefore best describes the occurrence of reversals in model M2. The Poisson process is the only one, among the selected random processes described by the different distribution functions tested, to be memoryless (see 
Section 3.4.2). This means that, once the dynamo establishes in either stable field polarity, a reversal can start at any time always with the same likelihood.

\subsubsection{Impact of the prior choice}

The previous statistical analysis assumed uniform priors for the distribution parameters. In order to test the robustness of the results presented above, we analyzed the impact of different prior choices on the posterior densities of the distribution parameters. Here the discussion is limited to the SPI durations obtained for the threshold combination $\mathrm{C}$ $\left(\mathcal{M}_{C}=0.4\right.$ and $\left.\mathcal{T}_{S}=0.8\right)$ and to the gamma distribution as a representative example. As in the analysis presented above, we fix in the following the prior for the rate parameter $\lambda$ to a uniform distribution within $1 / \tau$ and $1 / \mathcal{T}_{S}$, where $\tau$ is the total simulation time in units of the magnetic diffusion time.

Since the shape parameter $k$ is not expected to assume large values which would result in highly skewed distribution functions, other reasonable choices for the prior $\pi(k \mid$ Gamma $)$ are monotonically decreasing distributions. A linearly decreasing and a half normal prior have been selected for $k \in(0,5]$ (Figure 5.21, panel a).

Panel $b$ of Figure 5.21 illustrates, for each prior choice, the marginal posterior density of the gamma shape parameter $k$

$$
\mathrm{P}(k \mid \mathcal{P}, \text { Gamma })=\frac{\int_{\tau_{s}^{-1}}^{\mathcal{T}^{-1}} \mathrm{P}(k, \lambda \mid \mathcal{P}, \text { Gamma }) d \lambda}{\mathrm{P}(\mathcal{P} \mid \text { Gamma })},
$$

where $\mathrm{P}(\mathcal{P} \mid$ Gamma) is the evidence (3.94) of the SPI durations $\mathcal{P}$. The effect of the prior on the posterior distribution is rather mild and mainly consists in a shift towards lower $k$ values in the direction suggested by the prior. The marginal posterior densities are practically identical to normal distributions, thus confirming the dominance of the data likelihood on the priors. This test has been repeated for the different distribution functions selected and for the SPI durations from different combinations of $\mathcal{M}_{C}$ and $\mathcal{T}_{S}$ obtaining
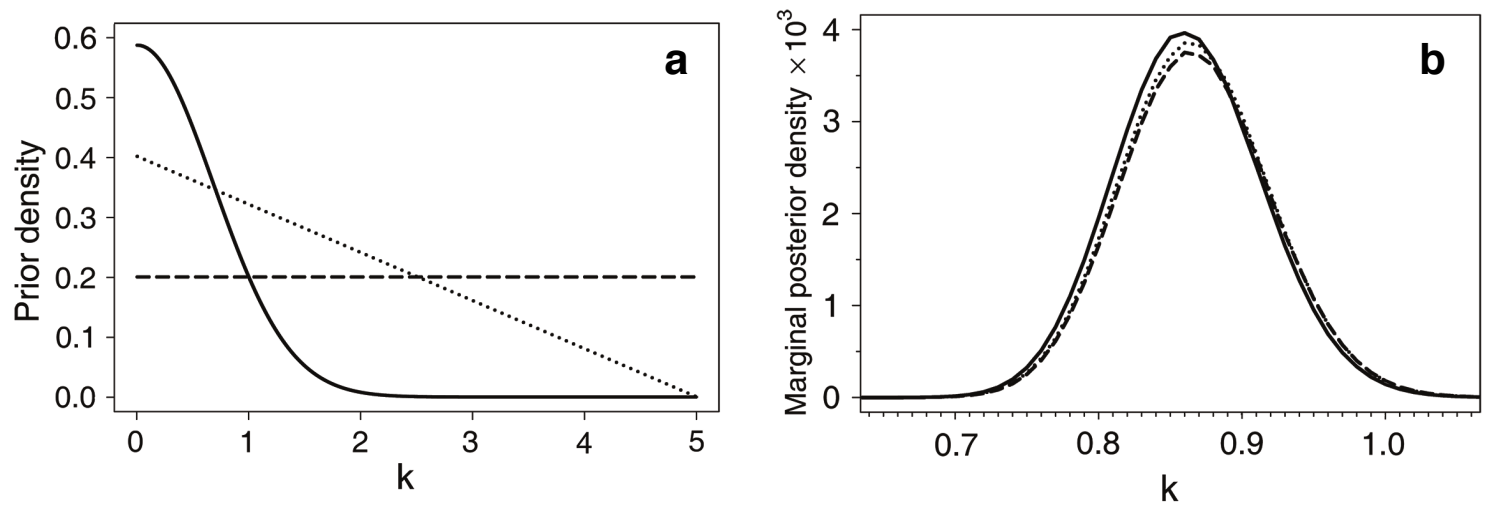

Figure 5.21: Panel a: Uniform (dashed curve), triangular (dotted curve) and half normal (solid curve) prior density functions used for the gamma shape parameter $k \in(0,5]$. The half normal prior has a half width at half maximum of $k=0.8$. Panel $b$ : Marginal posterior densities of $k$ (5.6) for the three selected prior distributions and for the stable polarity intervals $\mathcal{P}$ obtained for the combination $\mathcal{M}_{C}=0.4, \mathcal{T}_{S}=0.8$. 
similar results. This finally demonstrates the independence of our statistical results on the specific prior choice.

\subsubsection{Durations of reversals}

This section focuses on the distribution of the reversal durations $\mathcal{R}$ obtained for the different combinations of $\mathcal{M}_{C}$ and $\mathcal{T}_{S}$ explored. Figure 5.22 presents the results of the distribution fits of reversal durations. Only fits with KS and AD p-values larger than 5\% are shown. None of the proposed distribution functions can be rejected at the 5\% significance level for the threshold combinations explored.

For $\mathcal{M}_{C} \leq 0.2$, mainly gamma and Weibull distributions fit the reversal durations. Small $\mathcal{M}_{C}$ and $\mathcal{T}_{S}$ values promote shorter events, thus favoring the rapid monotonically decreasing gamma and Weibull PDFs with shape parameters lower than one. For $\mathcal{M}_{C}=$ 0.2 and larger $\mathcal{T}_{S}$ values, shorter stable polarity intervals are discarded together with the associated reversals (problem P4 of Section 5.3). This modifies the low end of the reversal duration PDF in favor of an enhanced probability for intermediate durations. Since the distribution profiles decrease less rapidly, the exponential can fit the reversal durations.

For $\mathcal{M}_{C} \geq 0.3$, skewed bell-shaped profiles of gamma, Weibull, log-normal and log-

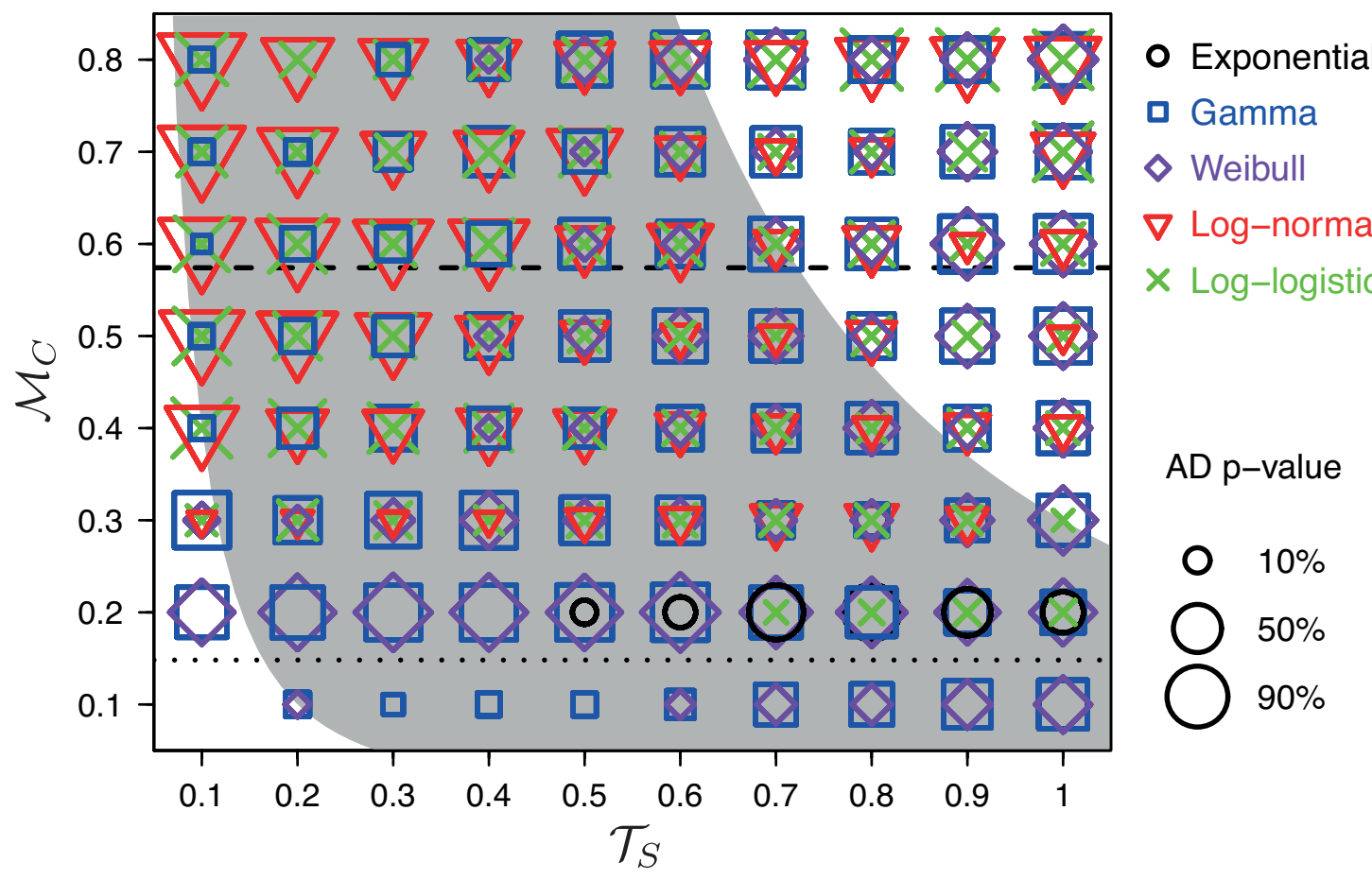

Figure 5.22: Anderson-Darling (AD) p-values of the distribution fits of the reversal durations $\mathcal{R}$ obtained for the threshold values $\mathcal{M}_{C}$ and $\mathcal{T}_{S}$ explored. The tested distribution functions are marked with different colored symbols (top right legend). Symbol sizes are scaled with the associated AD p-value (bottom right legend). Empty spaces occur where all the distribution functions can be rejected at the 5\% significance level. The dashed (dotted) horizontal line marks one (two) standard deviation(s) below the time-averaged dipole moment value. The gray background highlights parameter combinations expected to give reliable event definitions. 
logistic distributions fit the $\mathcal{R}$ sequences at the $95 \%$ confidence level. The best-fitting log-normal and log-logistic probability densities show slightly higher modes compared to the data (Figure 5.23, panel a). shows an example for $\mathcal{M}_{C}=0.3$ and $\mathcal{T}_{S}=0.2$. On the contrary, gamma and Weibull PDFs increase faster at lower reversal durations and underestimate the probabilities around the mode. Quantile-quantile plots show the reversal durations against the distribution quantiles (Figure 5.23, panel b) and reveal that the gamma distribution provides the closest overall match to the data with the highest AD p-value of $\alpha_{A D}=0.57$. The Weibull distribution severely underestimates the probabilities around the mode (Figure 5.23, panel a) which results in a lower AD p-value of $\alpha_{A D}=0.07$. While gamma and Weibull distributions provide a good description of the longer reversal durations, log-normal and log-logistic tend to overestimate such probabilities due to their heavy tails (Figure 5.23, panel b). The latter two distributions, however, cannot be rejected at the $5 \%$ significance level.

Though the p-value based analysis shows no clear preference for any particular model,
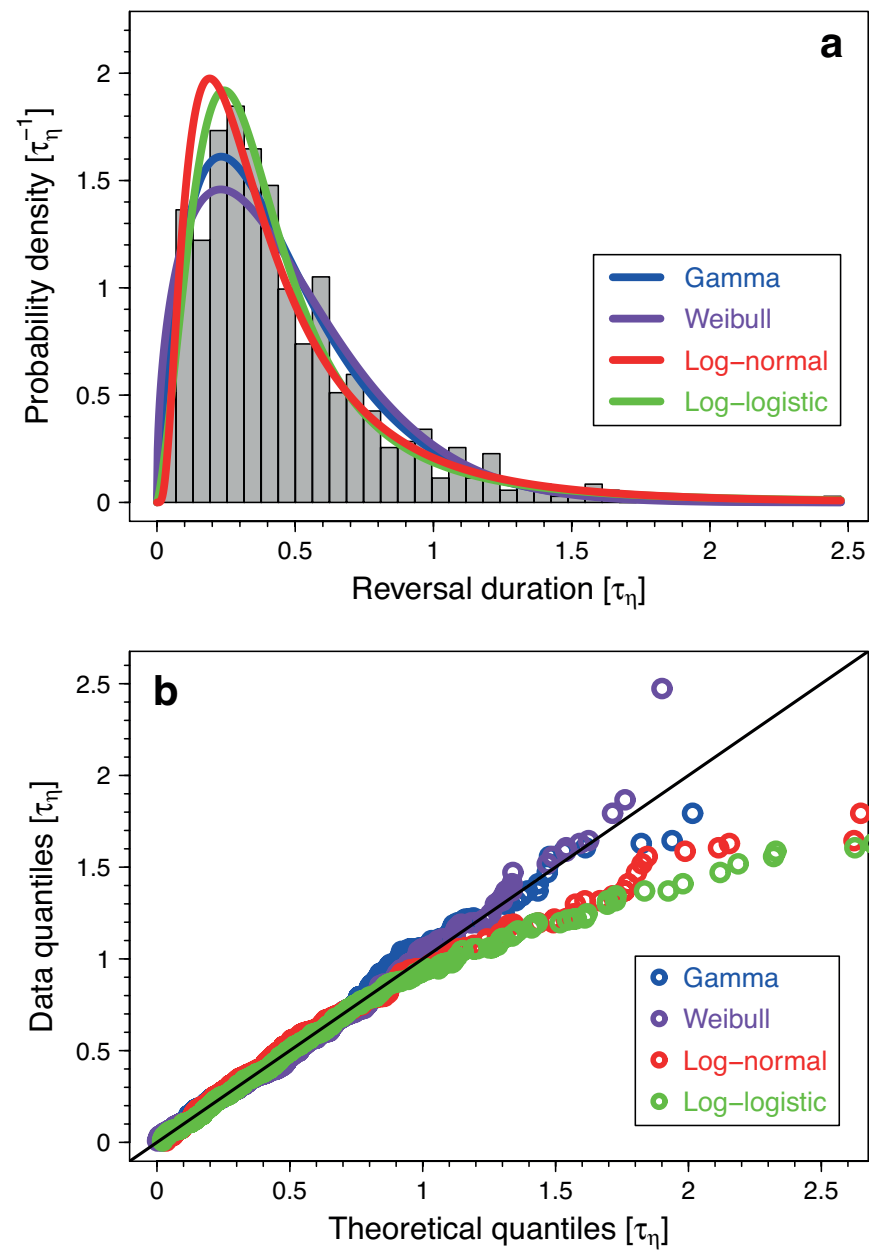

Figure 5.23: Panel a: Probability density of the reversal durations $\mathcal{R}$ obtained for $\mathcal{M}_{C}=0.3$ and $\mathcal{T}_{S}=0.2$ and relative best-fitting distribution functions (see the legend inset). Panel b: Quantile-quantile plot comparing the reversal durations $\mathcal{R}$ obtained for the threshold combination given above with the different best-fitting distribution functions (see the legend inset). 


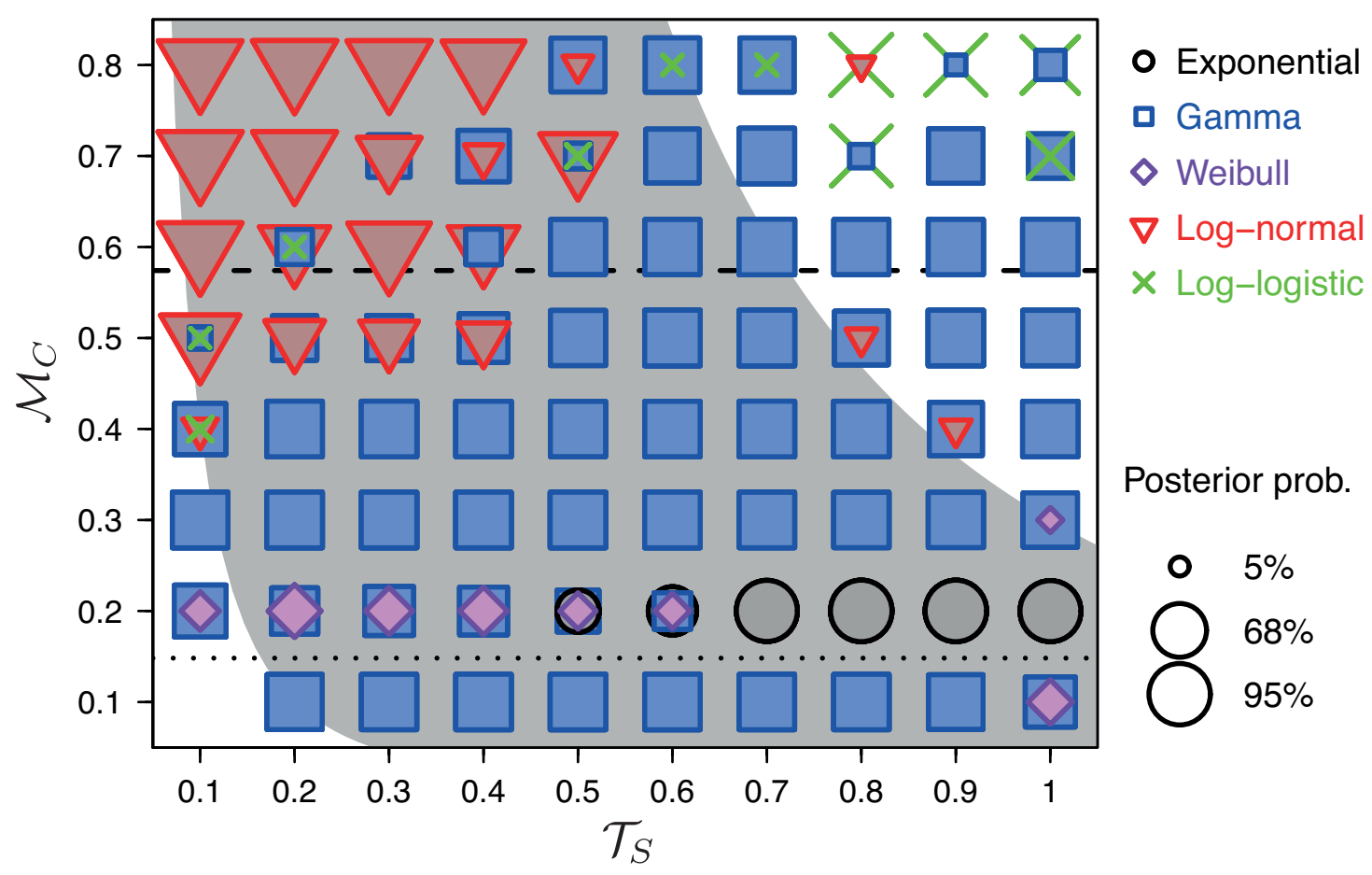

Figure 5.24: Bayesian posterior probabilities of the distribution functions (3.103) for the reversal durations $\mathcal{R}$ obtained for the threshold values $\mathcal{M}_{C}$ and $\mathcal{T}_{S}$ explored. The tested distribution functions are marked with different colored symbols (top right legend). Symbol sizes are scaled with the associated posterior probability (bottom right legend). Empty spaces occur where all the distribution functions have been rejected at the $5 \%$ significance level (cf. Figure 5.22). The dashed (dotted) horizontal line marks one (two) standard deviation(s) below the time-averaged dipole moment value. The gray background highlights parameter combinations expected to give reliable event definitions.

the fact that all best-fitting PDFs are bell-shaped and positively skewed represents a robust feature. Very short reversal transitions are indeed not very likely since these events cannot happen instantaneously. Once a polarity transition has started, the dipole field requires a finite time to decay and build up again into a new stable dipolar configuration.

Bayesian posterior probabilities permit to assess the relative likelihood of the statistical models tested. Figure 5.24 shows the posterior probabilities of each model for the threshold combinations explored. A definitive evidence in favor of the gamma distribution is found for the majority of $\mathcal{M}_{C}$ and $\mathcal{T}_{S}$ values. The exponential distribution is, however, the preferred model with posterior probabilities above $95 \%$ for $\mathcal{M}_{C}=0.2$ and $\mathcal{T}_{S} \geq 0.7$. Moreover, the log-normal distribution is promoted for large values of the dipole moment threshold, namely for $\mathcal{M}_{C} \geq 0.5$, and for moderate $\mathcal{T}_{S}$ values.

Since varying the dipole moment threshold directly affects the reversal durations $\mathcal{R}$, we studied the transition from low to high $\mathcal{M}_{C}$ values in more detail for $\mathcal{T}_{S}=0.2$. Figure 5.25 displays the distributions of reversal durations and the relative best-fitting gamma PDFs for $\mathcal{T}_{S}=0.2$ and three different $\mathcal{M}_{C}$ values. The gamma probability densities broaden when increasing $\mathcal{M}_{C}$ due to the systematically longer reversal durations defined (problem P1 of Section 5.3). Figure 5.26 shows significant variations in the posterior 


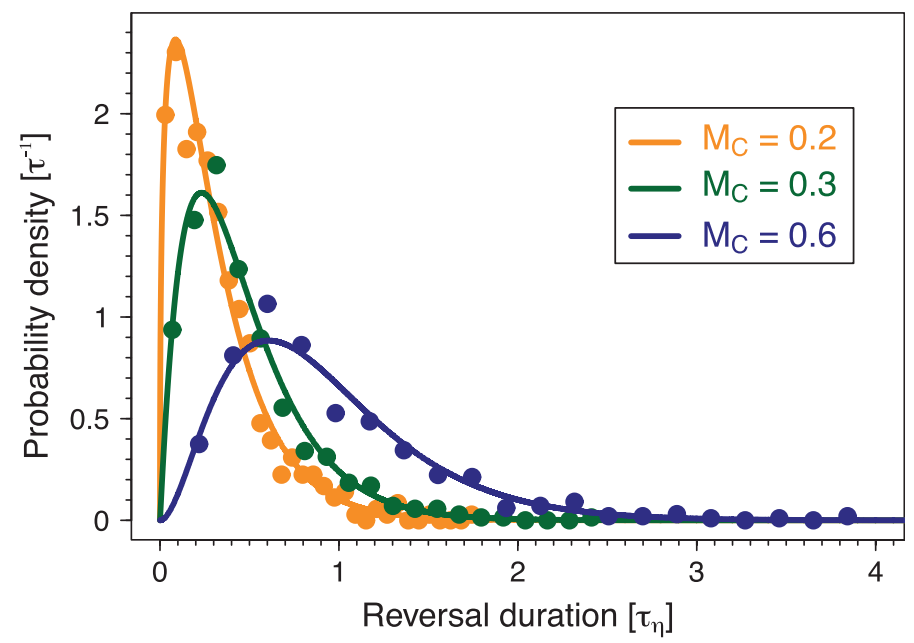

Figure 5.25: Distributions of reversal durations $\mathcal{R}$ obtained for a stable period $\mathcal{T}_{S}=0.2$ and three different values of the dipole moment threshold $\mathcal{M}_{C}$ (see the legend inset). Colored curves represent the respective best-fitting gamma distributions.

density of the gamma distribution parameters $\mathrm{P}\left(k, \lambda \mid \mathcal{R}\right.$, Gamma) for the three $\mathcal{M}_{C}$ values selected. When increasing $\mathcal{M}_{C}, \mathrm{P}(k, \lambda \mid \mathcal{R}$, Gamma) shifts towards larger values of the shape parameter $k$ and lower values of the rate $\lambda$. Differences in the gamma PDFs of Figure 5.25 for $\mathcal{M}_{C}=0.2$ and $\mathcal{M}_{C}=0.3$ are mainly due to the variation in the most likely value of $k$ which increases from $1.39 \pm 0.07$ to $2.03 \pm 0.11$ (Figure 5.26, panels a and b). The rate $\lambda$ stays practically constant between the two cases. An additional bias towards lower reversal durations is introduced by problem P2 (see Figure 5.11) when considering the low dipole moment threshold $\mathcal{M}_{C}=0.2$.

Increasing the critical dipole moment further to $\mathcal{M}_{C}=0.6$ leads to significantly longer reversals with a mean duration of $0.92 \tau_{\eta}$. The posterior density $\mathrm{P}(k, \lambda \mid \mathcal{R}$, Gamma) is consequently shifted towards higher values of the shape parameter with the most likely value of $k$ which equals 2.98 (cf. Figure 5.26, panel c). The rate at the posterior mode also significantly decreases to $\lambda=3.25 \tau_{\eta}^{-1}$. Problem P3 (Figure 5.11) is the main cause of the observed variations in the gamma distribution parameters. The impact of problem P3 on the reversal durations is significantly less severe at milder values of $\mathcal{M}_{C}$. The best-fitting gamma PDF for $\mathcal{M}_{C}=0.6$ (Figure 5.25, blue curve) is largely different from the cases with milder $\mathcal{M}_{C}$ values. The posterior probability of the gamma model $\mathrm{P}(\mathrm{Gamma} \mid \mathcal{R})$ diminishes from $99.9 \%$ for $\mathcal{M}_{C}=0.3$ to a milder $31.8 \%$ for $\mathcal{M}_{C}=0.6$. The posterior odds ratio (3.106) of the log-normal to the gamma distribution for the latter $\mathcal{M}_{C}$ value is of about 2, thus a mild evidence in favor of the former model is found (cf. also Figure 5.24). The paucity of short reversal durations in the $\mathcal{R}$ distribution for $\mathcal{M}_{C}=0.6$ and its somewhat more pronounced tail allow the log-normal to better describe the data. However, this preference is likely an artifact of problem P3. Figure 5.27 shows the posterior density of the log-normal parameters $\mu$ and $\sigma$. Since a bivariate normal distribution closely approximates this posterior density, the asymptotic limit of the data likelihood is reached even though the number of reversals is not as large as for smaller $\mathcal{M}_{C}$ values. The log-normal distribution parameters are practically uncorrelated since their correlation coefficient is as low as -0.012 . 

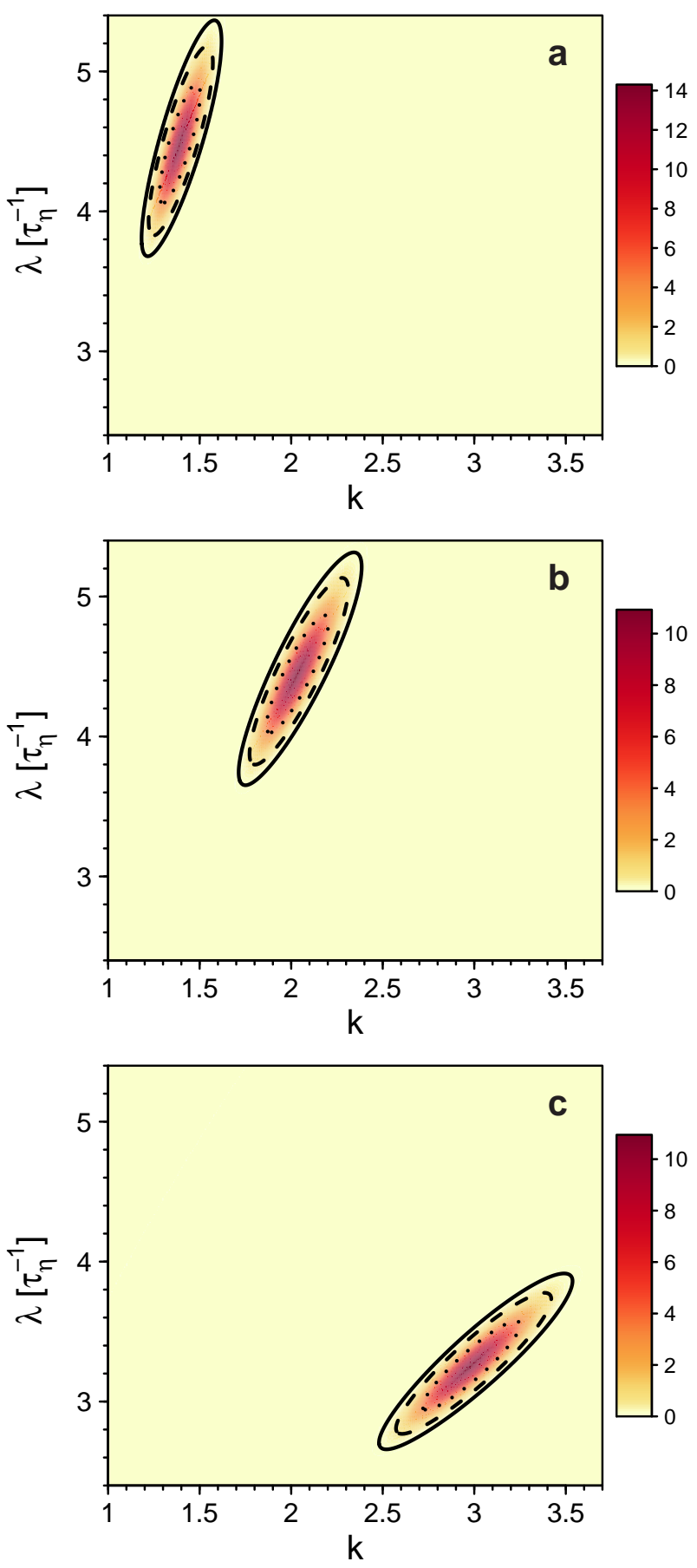

Figure 5.26: Bayesian posterior densities of the gamma distribution parameters for the reversal durations $\mathcal{R}$ obtained for $\mathcal{M}_{C}=0.2$ (panel a), $\mathcal{M}_{C}=0.3$ (panel b) and $\mathcal{M}_{C}=0.6$ (panel c). and a fixed stable period of $\mathcal{T}_{S}=0.2$. Iso-contour lines represent the $68 \%, 95 \%$ and $99.7 \%$ highest posterior density intervals.

Larger values of $\mathcal{T}_{S}$ also impact the distribution of reversal durations especially at moderate values of the dipole moment threshold $\mathcal{M}_{C}$. For example, a transition from a gamma to an exponential distribution is observed for $\mathcal{M}_{C}=0.2$ in Figure 5.24. In order to study such transition, we selected the three $\mathcal{T}_{S}$ values of $0.2,0.5$ and 0.8 . Figure 


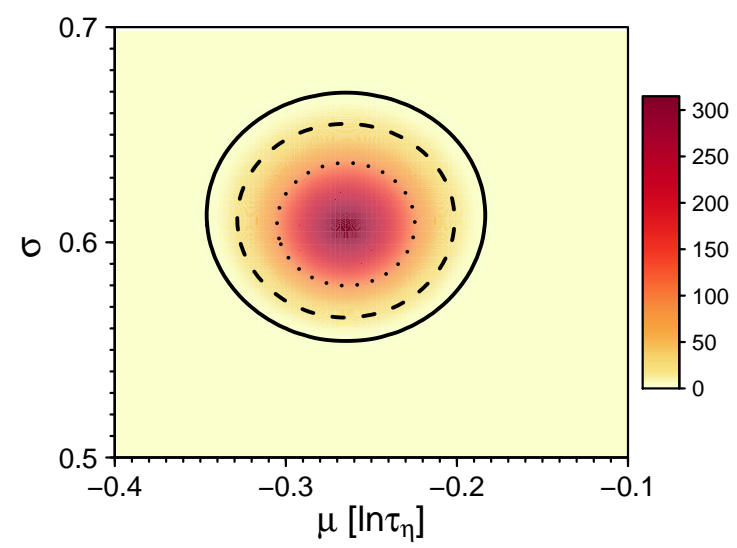

Figure 5.27: Bayesian posterior density of the log-normal distribution parameters $\mu$ and $\sigma$ for the reversal durations $\mathcal{R}$ obtained for the threshold combination $\mathcal{M}_{C}=0.6, \mathcal{T}_{S}=0.2$. Iso-contour lines represent the $68 \%, 95 \%$ and $99.7 \%$ highest posterior density intervals.

5.28 shows time series of the dipole tilt and dipole moment for each of the selected cases, while the respective distributions of reversal durations are depicted in Figure 5.29 together with the distribution functions having the highest Bayesian posterior probability. Increasing $\mathcal{T}_{S}$, the distribution functions broaden as expected and shorter reversals become less likely.

Since $\mathcal{M}_{C}$ is relatively low, small dipole moment variations in proximity of reversal transitions likely define excursions (problem P2 of Figure 5.11). This situation frequently occurs with the lowest $\mathcal{T}_{S}$ value of 0.2 and a typical example can be found in Figure 5.28 (panel a) at the time $t \simeq 2896 \tau_{\eta}$. When increasing $\mathcal{T}_{S}$ to 0.5 , the associated reversal and grand excursion merge into a longer reversal (Figure 5.28, panel b). The short end of the reversal duration distribution is thus depopulated while intermediate and long durations become more likely. Excursions should be typically regarded as part of the reversal transition when the dipole moment does not recover significantly enough between the two events. However, the combination of small $\mathcal{M}_{C}$ and large $\mathcal{T}_{S}$ overemphasizes this effect. The posterior odds ratio (3.106) of the gamma to the exponential distribution relative to the combination $\mathcal{M}_{C}=0.2$ and $\mathcal{T}_{S}=0.5$ is 1.3 and therefore the two models are almost equally likely. The best-fitting gamma distribution shows a good agreement with the data as expected (Figure 5.29, blue curve).

The marginal posterior density of the gamma shape parameter $k$

$$
\mathrm{P}(k \mid \mathcal{R}, \text { Gamma })=\frac{\int_{\tau^{-1}}^{10} \mathrm{P}(k, \lambda \mid \mathcal{R}, \text { Gamma }) d \lambda}{\mathrm{P}(\mathcal{R} \mid \text { Gamma })}
$$

points out that the value $k=1$ for which the gamma coincides with the exponential is excluded from the $95 \%$ credibility region for $\mathcal{T}_{S}=0.5$ (Figure 5.30, panel a). This explains the very mild preference of the gamma distribution over the exponential by the Bayesian analysis in this case. A comparison of the posterior densities $\mathrm{P}(k, \lambda \mid \mathcal{R}, \mathrm{Gamma})$ for the combination $\mathcal{M}_{C}=0.2$ and $\mathcal{T}_{S}=0.2$ (Figure 5.26, panel a) and for $\mathcal{M}_{C}=0.2$ and $\mathcal{T}_{S}=0.5$ (Figure 5.30, panel c) reveals that the shape parameter $k$ slightly shifts towards one and the rate $\lambda$ significantly decreases when increasing $\mathcal{T}_{S}$.

As $\mathcal{T}_{S}$ increases further to 0.8 , 'proper' SPIs are discarded and considered as long 


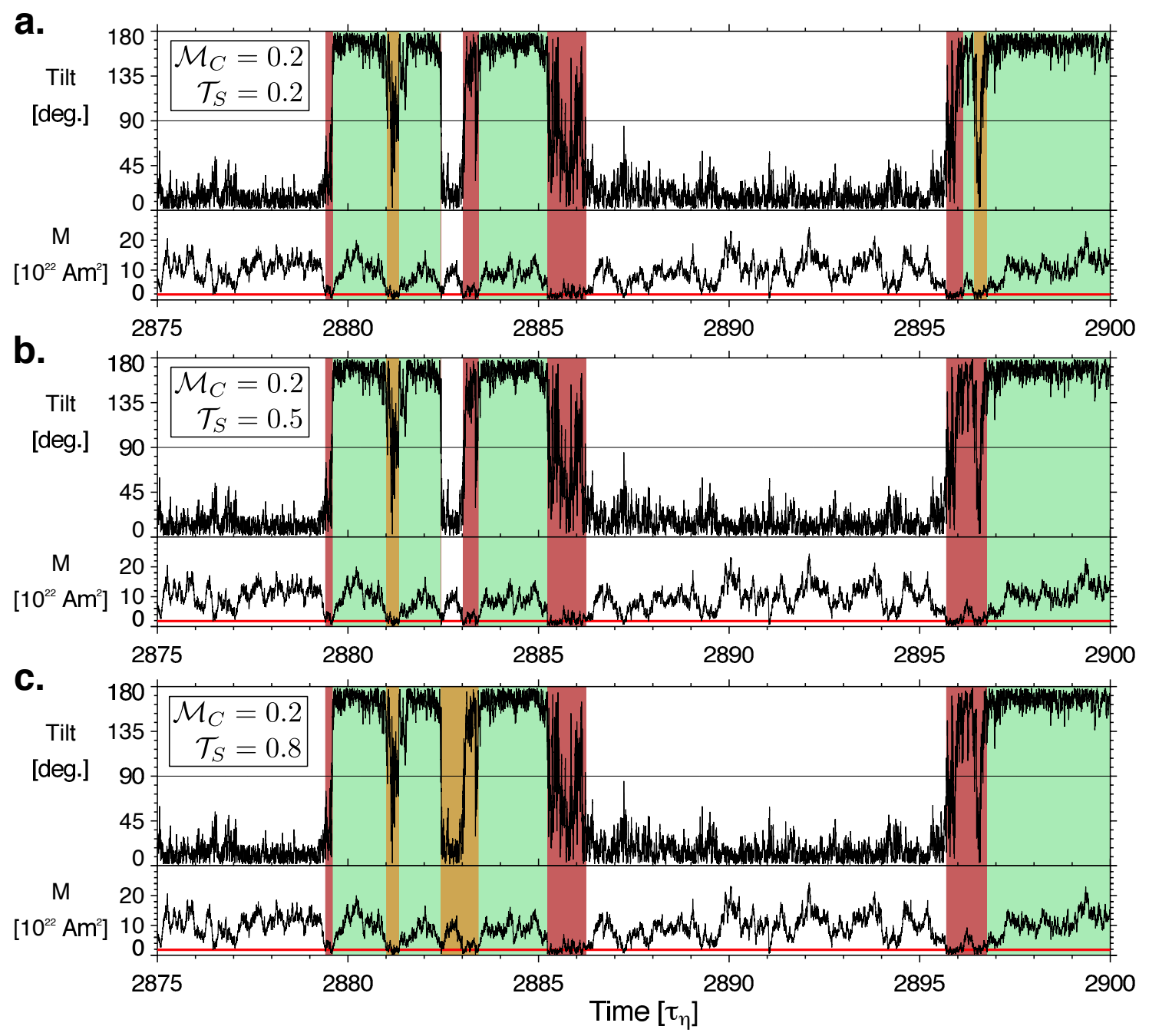

Figure 5.28: Effect of increasing the stable period $\mathcal{T}_{S}$ on the counting of reversals and grand excursions. Time series of dipole tilt and dipole moment are shown in the upper and lower sub-panels respectively for $\mathcal{T}_{S}=0.2$ (panel a), $\mathcal{T}_{S}=0.5$ (panel b) and $\mathcal{T}_{S}=$ 0.8 (panel c). The dipole moment threshold is fixed to $\mathcal{M}_{C}=0.2$. Red and orange background colors highlight reversals and grand excursions respectively, while opposite stable polarities are marked in white and green.

grand excursions (problem P4 of Figure 5.11). A typical example of such behavior is shown in Figure 5.28 (panel c) at the time $t \simeq 2883 \tau_{\eta}$. Since reversals associated with the discarded SPIs are more likely short events, the distribution of reversal durations is more severely affected at its low end (cf. Figure 5.29). The exponential distribution can therefore describe the reversal durations and, having a posterior probability of $95 \%$, the evidence in favor of this model is high. The best-fitting exponential PDF remarkably agrees with the data as expected (red curve in Figure 5.29). The gamma distribution achieves a posterior probability as modest as $3 \%$. Contrary to the threshold value $\mathcal{T}_{S}=0.5$ analyzed before, the marginal posterior density of $k$ 5.7 for $\mathcal{T}_{S}=0.8$ shows that the value $k=1$ lies in the $68 \%$ credibility interval (Figure 5.30, panel d). The Bayesian analysis favors the exponential distribution which achieves the fit with only one rather than two 


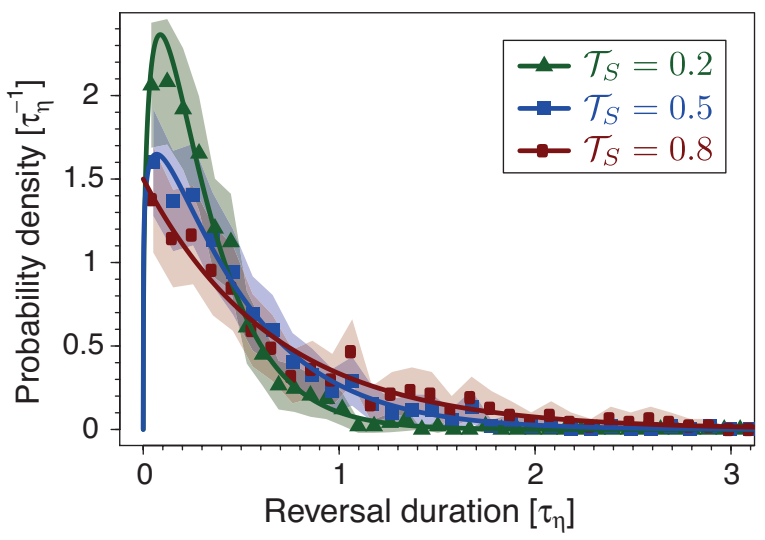

Figure 5.29: Effect of increasing the stable period $\mathcal{T}_{S}$ (see the legend inset for the explored values) on the distribution of reversal durations $\mathcal{R}$ obtained for $\mathcal{M}_{C}=0.2$. Color shaded areas mark the $95 \%$ confidence intervals of the binned distributions. Solid curves represent the best-fitting gamma $\left(\mathcal{T}_{S}=0.2\right.$ and $\left.\mathcal{T}_{S}=0.5\right)$ and exponential $\left(\mathcal{T}_{S}=0.8\right)$ distributions.
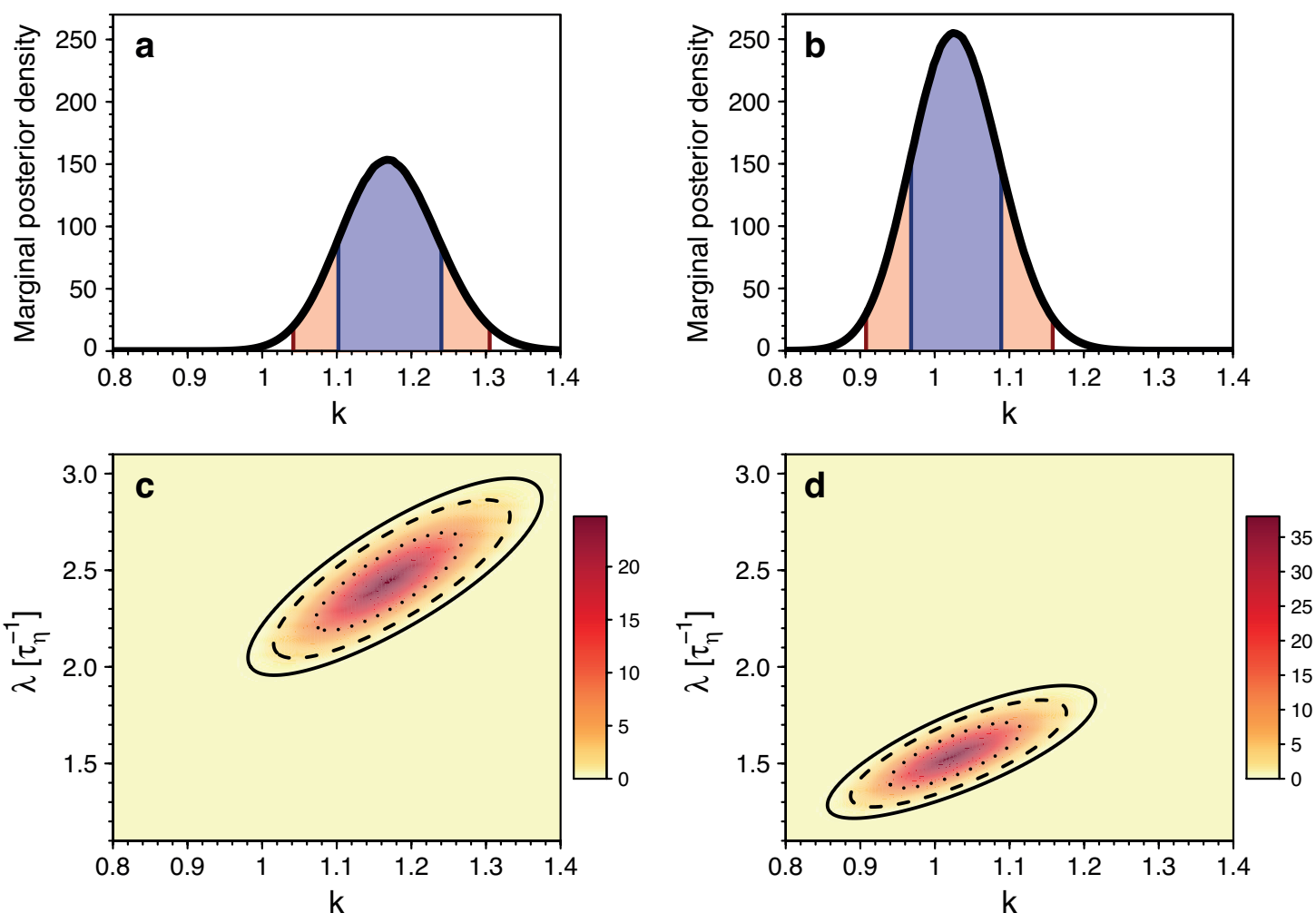

Figure 5.30: Posterior densities of the gamma distribution parameters $k$ and $\lambda$ (bottom panels) and associated marginal posterior densities of the shape parameter (5.7) (top panels) for the reversal durations $\mathcal{R}$ obtained for $\mathcal{M}_{C}=0.2$ and a stable period $\mathcal{T}_{S}=0.5$ (left panels) and $\mathcal{T}_{S}=0.8$ (right panels). Blue and red areas mark $68 \%$ and $95 \%$ credibility intervals respectively (panels a and b), while the iso-contour lines represent the 68\%, 95\% and $99.7 \%$ highest posterior density intervals (panels $\mathrm{c}$ and d). 
parameters.

Having attributed the transitions from gamma to log-normal (exponential) distributions to the too large $\mathcal{M}_{C}\left(\mathcal{T}_{S}\right)$ values, we can firmly conclude that the gamma distribution is a robust model for the description of reversal durations with reliable threshold values.

\subsubsection{Durations of grand excursions}

The distributions of the grand excursion durations $\mathcal{E}$ show strong similarities with those obtained for the reversal durations $\mathcal{R}$ in the previous section. Figure 5.31 presents the AD $\mathrm{p}$-values of the different distribution fits of the grand excursion duration obtained for the threshold combinations explored.

For $\mathcal{M}_{C} \leq 0.2$, mainly gamma and Weibull distributions fit the data at the $95 \%$ confidence level. An analogous situation has been observed for reversal durations at low $\mathcal{M}_{C}$ values (Figure 5.22). Signatures for an exponential fit occur at $\mathcal{M}_{C}=0.2$ for few $\mathcal{T}_{S}$ values. Contrary to reversal durations, there is no transition towards the exponential at larger $\mathcal{T}_{S}$ values. For $\mathcal{M}_{C} \geq 0.3$, only the exponential model can be rejected at the given significance level while the remaining distributions are all acceptable.

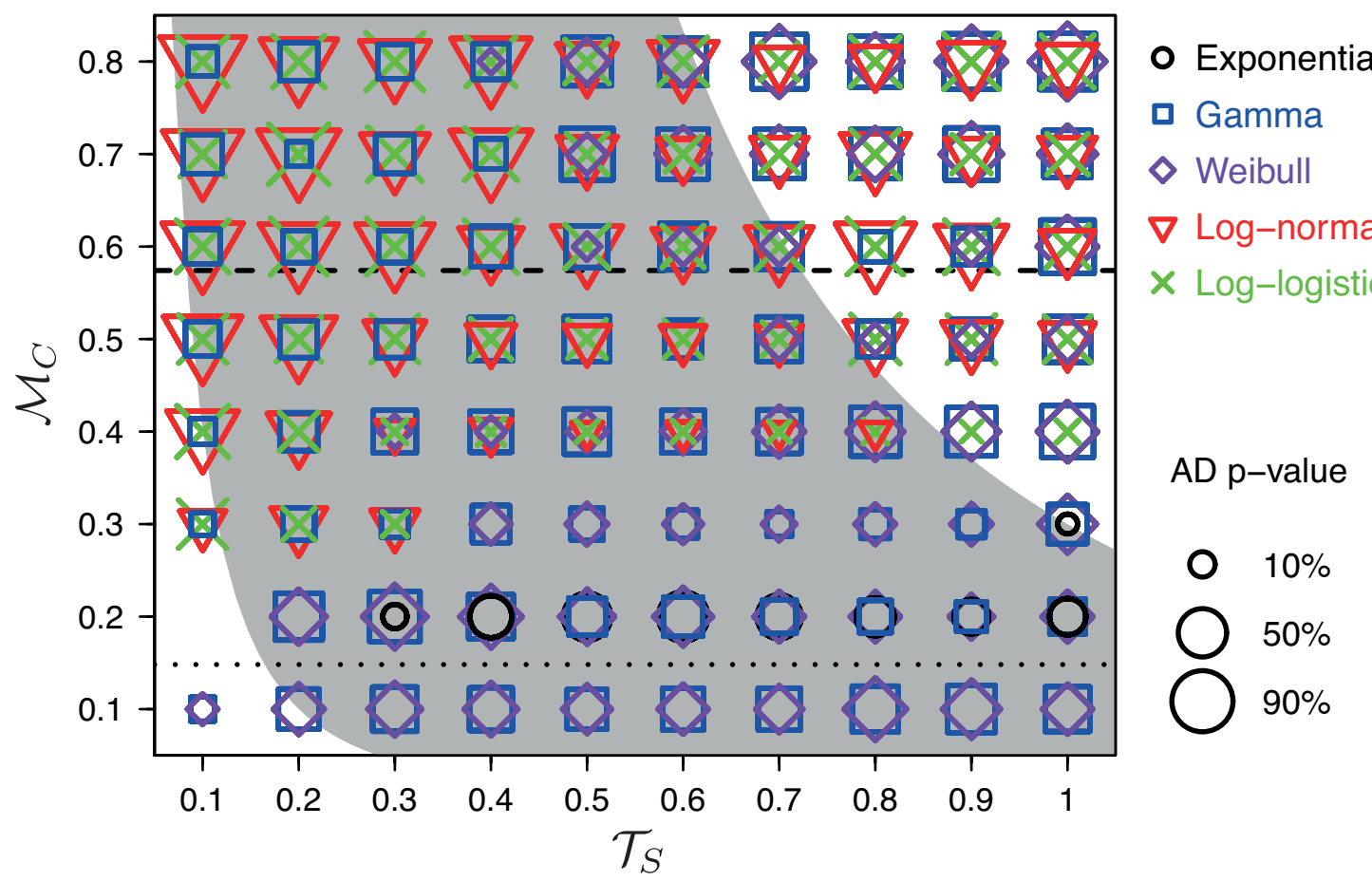

Figure 5.31: Anderson-Darling (AD) p-values of the distribution fits of the excursion durations $\mathcal{E}$ obtained for the threshold values $\mathcal{M}_{C}$ and $\mathcal{T}_{S}$ explored. The tested distribution functions are marked with different colored symbols (top right legend). Symbol sizes are scaled with the associated AD p-value (bottom right legend). Empty spaces occur where all the distribution functions can be rejected at the 5\% significance level. The dashed (dotted) horizontal line marks one (two) standard deviation(s) below the time-averaged dipole moment value. The gray background highlights parameter combinations expected to give reliable event definitions. 

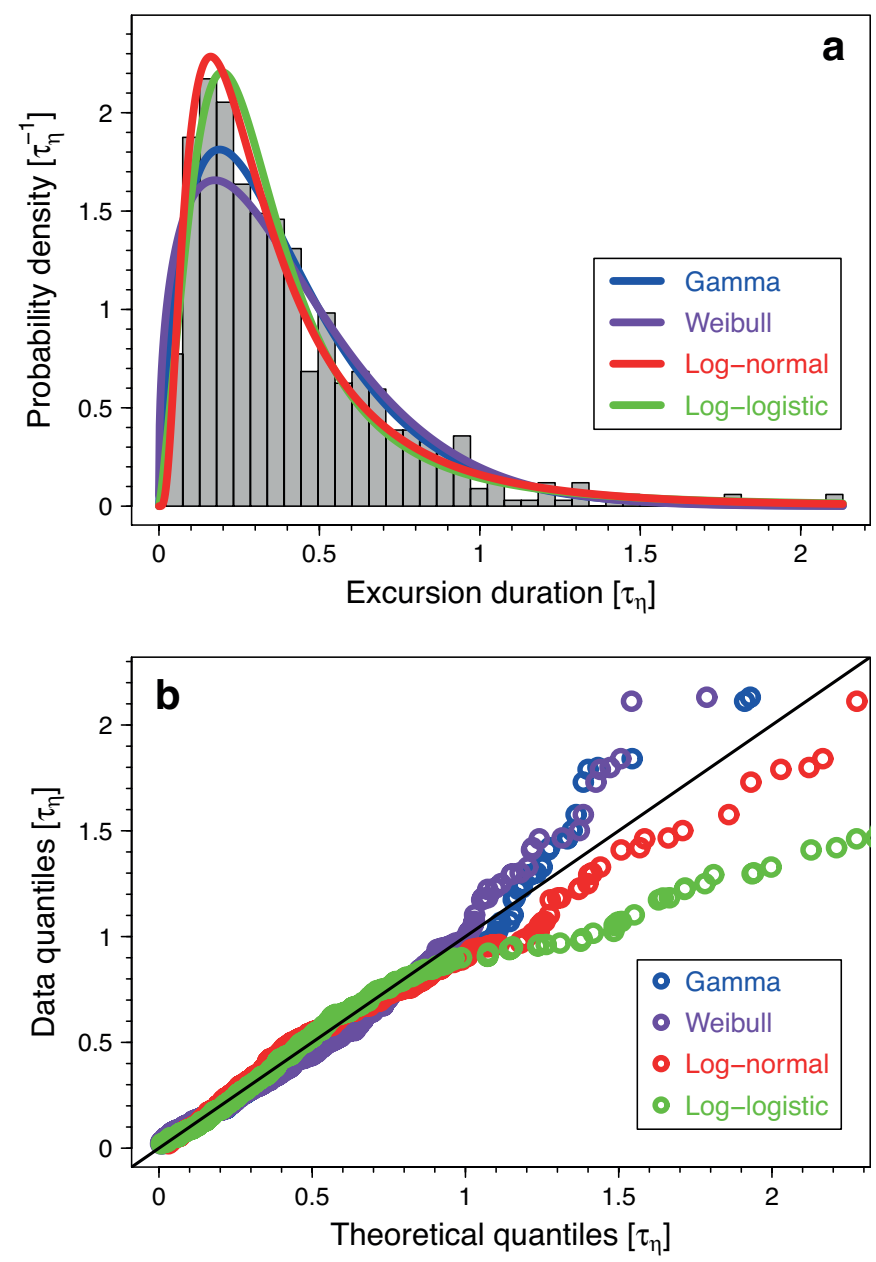

Figure 5.32: Panel a: Probability density of the excursion durations $\mathcal{E}$ obtained for $\mathcal{M}_{C}=$ 0.3 and $\mathcal{T}_{S}=0.2$ and relative best-fitting distribution functions (see the legend inset). Panel b: Quantile-quantile plot comparing the excursion durations $\mathcal{E}$ obtained for the threshold combination given above with the different best-fitting distribution functions (see the legend inset).

Figure 5.32 (panel a) exhibits the histogram of the grand excursion durations for the combination $\mathcal{M}_{C}=0.3$ and $\mathcal{T}_{S}=0.2$ with the relative best-fitting distribution functions. As for reversal durations, it is difficult to assess which model better describes the data. Log-normal and log-logistic PDFs seem to closely describe the rapid increase at small durations. On the other hand, gamma and Weibull distributions better describe the data at intermediate excursion durations, while the log-normal and log-logistic underestimate the respective probabilities. Excursions longer than the magnetic diffusion time $\tau_{\eta}$ are poorly captured by all the different models (Figure 5.32, panel b). The log-normal and log-logistic distributions, due to their heavy tails, tend to overestimate the probabilities of occurrence of the longer events, while gamma and Weibull underestimate the respective likelihoods.

Since four different models fit the excursion durations obtained for the reliable threshold combinations, no particular model can be selected in the frequentist approach. We therefore computed the Bayesian posterior model probabilities for the threshold combi- 


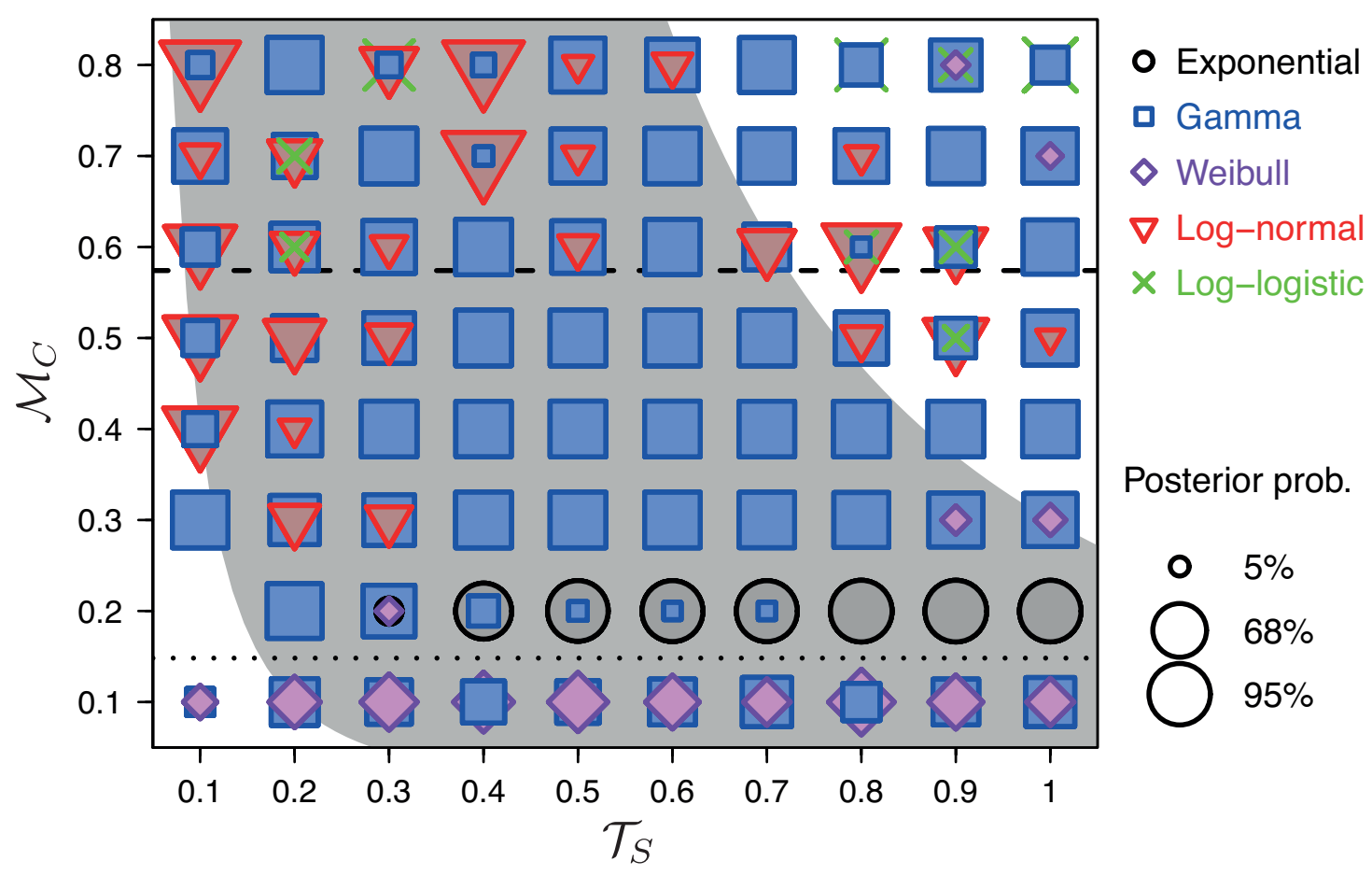

Figure 5.33: Bayesian posterior probabilities of the distribution functions (3.103) for the excursion durations $\mathcal{E}$ obtained for the threshold values $\mathcal{M}_{C}$ and $\mathcal{T}_{S}$ explored. The tested distribution functions are marked with different colored symbols (top right legend). Symbol sizes are scaled with the associated posterior probability (bottom right legend). Empty spaces occur where all the distribution functions have been rejected at the $5 \%$ significance level (cf. Figure 5.31). The dashed (dotted) horizontal line marks one (two) standard deviation(s) below the time-averaged dipole moment value. The gray background highlights parameter combinations expected to give reliable event definitions.

nations explored and the results are displayed in Figure 5.33. The gamma distribution is the most likely model for the vast majority of $\mathcal{M}_{C}$ and $\mathcal{T}_{S}$ values. In particular, posterior gamma probabilities larger than $95 \%$ are obtained for intermediate values of $\mathcal{M}_{C}$ and $\mathcal{T}_{S}$. Variations of the posterior model probabilities in Figure 5.33 seem also to be similar to those obtained for reversal durations (cf. Figure 5.24). For example, a strong evidence in favor of the exponential distribution is achieved for $\mathcal{M}_{C}=0.2$ and larger $\mathcal{T}_{S}$ values. Moreover, increasing $\mathcal{M}_{C}$ at low $\mathcal{T}_{S}$ values renders the log-normal distribution more likely. The posterior odds ratios (3.106) of the log-normal to the gamma distribution are, however, mildly larger than one only for the combinations of $\mathcal{T}_{S}=0.1$ and moderate to high $\mathcal{M}_{C}$ values and for few other extreme parameter combinations. There is no decisive evidence in favor of the log-normal distribution for grand excursion durations contrary to reversal durations. We refer to Section 5.4 .2 for a detailed explanation of the type of changes experienced by the distribution profiles when increasing the dipole moment threshold $\mathcal{M}_{C}$ or the stable period $\mathcal{T}_{S}$. As will be demonstrated in the following section, the estimated distribution parameters are also very similar to those obtained for reversal durations.

We can conclude that the grand excursion durations $\mathcal{E}$, similarly to the reversal du- 
rations $\mathcal{R}$, are gamma distributed with shape parameters $k>1$. The question whether reversals and excursions are actually equivalent events will be addressed in the following section.

\subsubsection{Equivalence of reversals and grand excursions}

In the previous sections, we demonstrated that both reversal durations $\mathcal{R}$ and grand excursion durations $\mathcal{E}$ from the dynamo model M2 are best described by a gamma distribution. Strong similarities in the variation of the estimated distribution parameters obtained for the combinations of $\mathcal{M}_{C}$ and $\mathcal{T}_{S}$ explored may also suggest that reversals and grand excursions are equivalent events. We further support this hypothesis in the following.

Figure 5.34 displays the number of grand excursions $N_{E}$ against the number of reversals $N_{R}$ for each combination of $\mathcal{M}_{C}$ and $\mathcal{T}_{S}$ explored. Filled circles represent combinations of $\mathcal{M}_{C}$ and $\mathcal{T}_{S}$ where the event definitions are reliable (see Section 5.4.1) and they therefore belong to the region highlighted in gray in Figures 5.22 and 5.31. The open symbols in Figure 5.34 mark threshold combinations outside such region. The number of grand excursions seems to be systematically larger for the reliable event definitions. The largest difference is of $15 \%$ in favor of grand excursions and is obtained for the combination $\mathcal{M}_{C}=0.3, \mathcal{T}_{S}=0.1$. A linear least-squares fit of the events number provides $N_{E}=-91+1.27 N_{R}$ (red line in Figure 5.34). This difference can be attributed to the choice of the lower dipole moment threshold, used to define grand excursions, of $\mathcal{M}_{C}^{\mathcal{E}}=0.2$ (Section 5.3.1). Note that using a lower value of $\mathcal{M}_{C}^{\mathcal{E}}$ would reduce $N_{E}$.

Similarities between reversals and grand excursions can be further assessed by comparing the estimated gamma distribution parameters of the event durations (Sections 5.4.2 and 5.4.3). Figure 5.35 shows the maximum likelihood estimates of the gamma distribution parameters for the reversal durations $\mathcal{R}$ against those obtained for the grand excursion

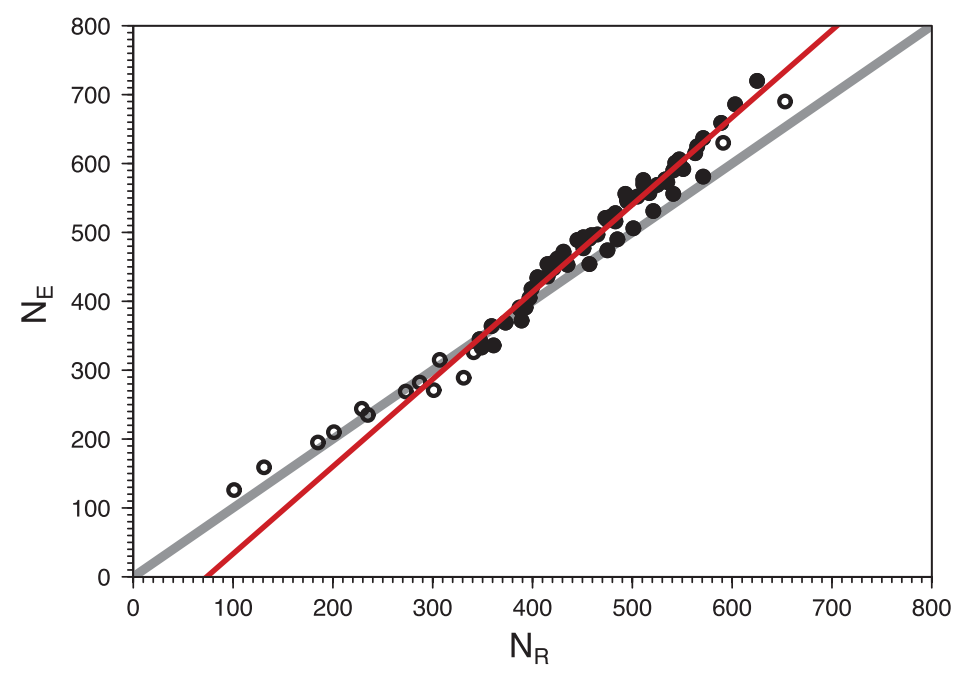

Figure 5.34: Number of grand excursions $N_{E}$ against number of reversals $N_{R}$ obtained for all the different threshold combinations $\mathcal{M}_{C}$ and $\mathcal{T}_{S}$ explored. Filled symbols mark the threshold combinations with reliable event definitions, while open symbols refer to a biased event counting (see Section 5.4.1). The grey background line indicates $N_{E}=N_{R}$, while the linear least-squares fit of the filled points is shown in red. 

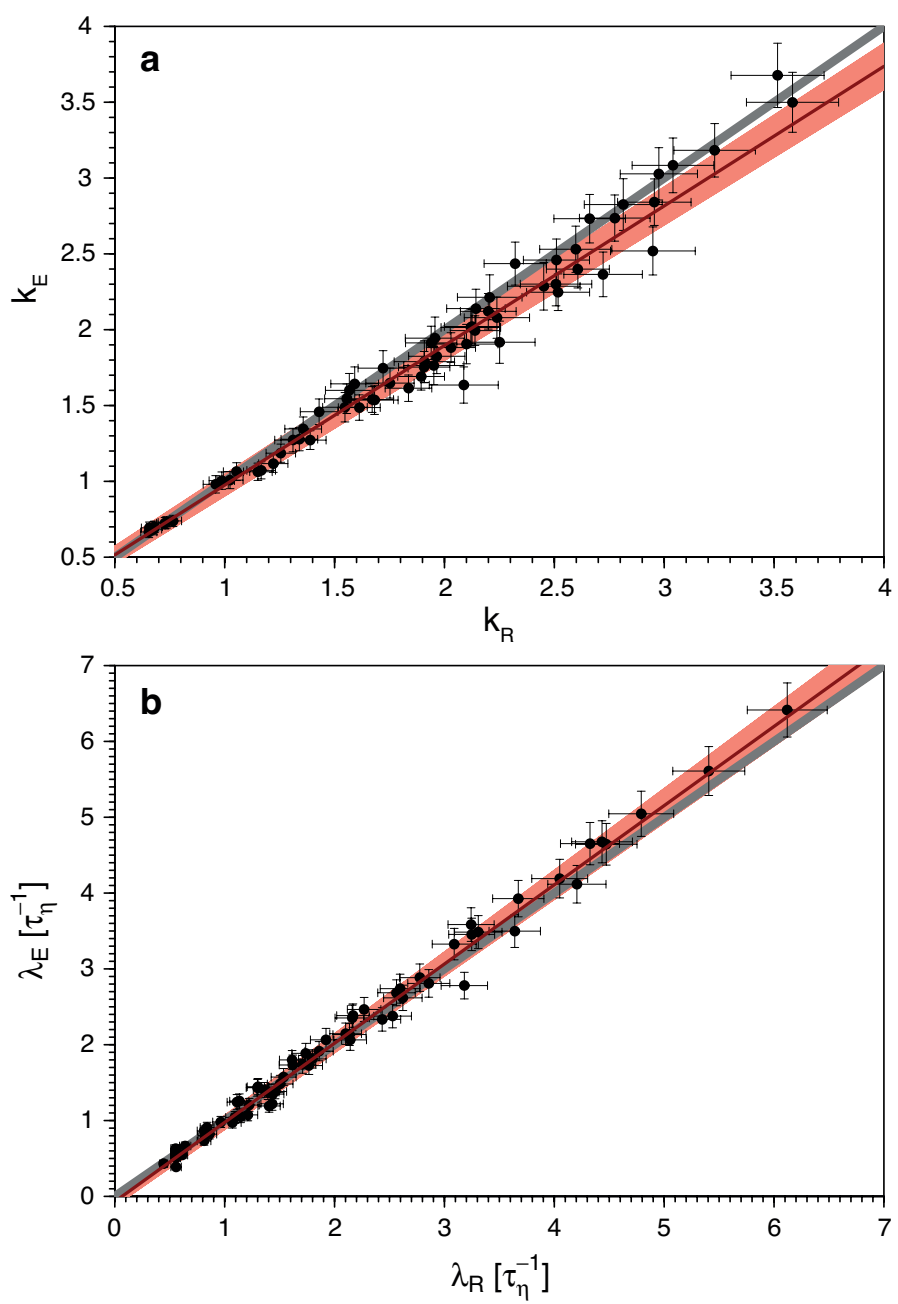

Figure 5.35: Estimated gamma distribution parameters (shape $k$ in panel a and rate $\lambda$ in panel b) for reversal durations ( subscript $_{R}$ ) and grand excursion durations (subscript ${ }_{E}$ ). Each plotted symbol refers to a combination of $\mathcal{M}_{C}$ and $\mathcal{T}_{S}$ explored. Error bars represent the $68 \%$ confidence intervals of the relative estimate. The grey background line in panel a (panel b) indicates $k_{E}=k_{R}\left(\lambda_{E}=\lambda_{R}\right)$. Linear least-squares fits are shown in red with the color shaded backgrounds representing the respective $95 \%$ confidence intervals.

durations $\mathcal{E}$. Only combinations of $\mathcal{M}_{C}$ and $\mathcal{T}_{S}$ values where the gamma distribution fits both type of events at the $95 \%$ confidence level are shown. Each symbol in Figure 5.35 refers to a threshold combination with reliable event definitions. Horizontal and vertical error bars show standard errors, i.e. the $68 \%$ confidence intervals calculated from (3.82), of the relative estimates. Weighted linear least-squares fits are displayed in red with the associated $95 \%$ confidence intervals.

The estimated gamma shape parameters for reversals $k_{R}$ seem to be slightly larger than those of grand excursions $k_{E}$ (Figure 5.35, panel a). The linear least-squares fit provides $k_{E} \sim(0.92 \pm 0.03) k_{R}$ thus confirming the presumed trend. This estimate is, however, partly biased by the large scatter introduced by the threshold combinations with the higher $\mathcal{M}_{C}$ and $\mathcal{T}_{S}$ values which have the larger errors due to their small number of events. The line $k_{E}=k_{R}$ lies indeed within the $95 \%$ confidence interval at lower shape parameter values 


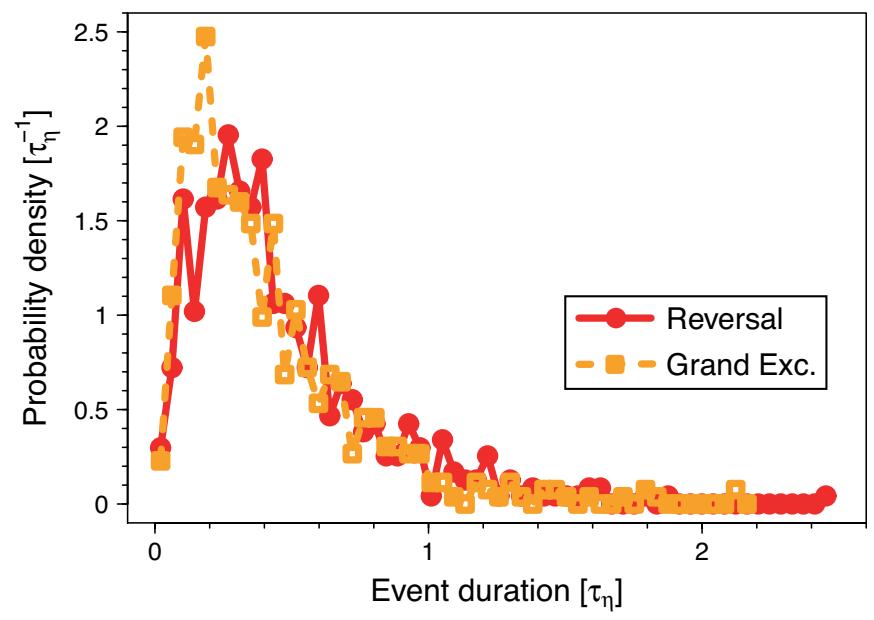

Figure 5.36: Distribution of the reversal durations $\mathcal{R}$ and grand excursion durations $\mathcal{E}$ obtained for the threshold combination $\mathcal{M}_{C}=0.3, \mathcal{T}_{S}=0.2$.

where the statistics are well constrained.

The estimated gamma rate parameters for the durations of reversals $\lambda_{R}$ and grand excursions $\lambda_{E}$ seem to mildly favor the latter type of events (Figure 5.35, panel b). The least-squares fit provides $\lambda_{E} \sim(1.04 \pm 0.03) \lambda_{R}$, thus confirming the agreement between the estimated rates. The lower shape parameter values and the somewhat larger rates of grand excursions as compared to reversals are consistent with the higher number of grand excursions identified in the simulation run.

For the sake of completeness, the distributions of reversal and grand excursion durations for the combination $\mathcal{M}_{C}=0.3, \mathcal{T}_{S}=0.2$ is shown in Figure 5.36 as an example. The distributions of the two event types are very similar as expected. The higher number of excursions at lower durations and the larger mean reversal duration is fitted with gamma distributions having a slightly higher rate in favor of excursions $\left(\lambda_{E}=4.68 \pm 0.28\right.$ and $\left.\lambda_{R}=4.43 \pm 0.28\right)$. Moreover, the narrower grand excursion distribution profile results in a somewhat lower estimated shape $\left(k_{E}=1.88 \pm 0.10\right.$ and $\left.k_{R}=2.03 \pm 0.11\right)$.

We can conclude that the statistical differences between reversals and grand excursions in model M2 do not support a clear separation between the two event types. The similarities found in the respective events numbers imply that the dynamo, after entering a transitional period, recovers its dipole field in the same polarity as before (excursion) or in the opposite one (reversal) with equal chance. Moreover, we found analogous estimates of the gamma distribution parameters relative to each type of event in a wide range of threshold combinations. This implies that reversals and grand excursions have a similar temporal dynamics during the respective low dipole intensity phases. It is important to recall that only grand excursions have been considered here. If the constraint on the dipole strength during excursions $\left(\mathcal{M}_{C}^{\mathcal{E}}\right)$ is relaxed, the so-called dipole dips are included (see Section 5.3). In such situation, 'excursion-like' events are much more numerous than reversals. Taking into account all excursions gives estimates of the gamma shape parameters significantly lower than those of reversals, while rate parameters are lower for the latter type of events as expected. 


\subsection{Stable polarity intervals from a paleomagnetic point of view}

Paleomagnetic reversal chronologies are based exclusively on the polarity of the magnetic field (see Section 1.4.4). Contrary to the analysis presented above, bounding reversals therefore contribute to the duration of each stable polarity epoch. A separation of reversal and SPI durations based on the dipole moment would only be possible for the few most recent events where intensity estimates are actually available. However, we can mimic the paleomagnetic data set by defining polarity epochs which include half of the duration of each bounding reversal:

$$
C=\left\{\frac{\Delta t_{i}^{R}}{2}+\Delta t_{i}^{P}+\frac{\Delta t_{i+1}^{R}}{2}\right\}_{i=1}^{N_{R}-1} .
$$

For consistency with the nomenclature adopted in paleomagnetism, we refer hereafter to the polarity epochs $C$ to as chrons.

Similarly to the analysis of the SPIs presented above, we fitted left truncated distribution functions by $\mathcal{T}_{S}$ to the chrons $C$. Figure 5.37 summarizes the results of the distri-

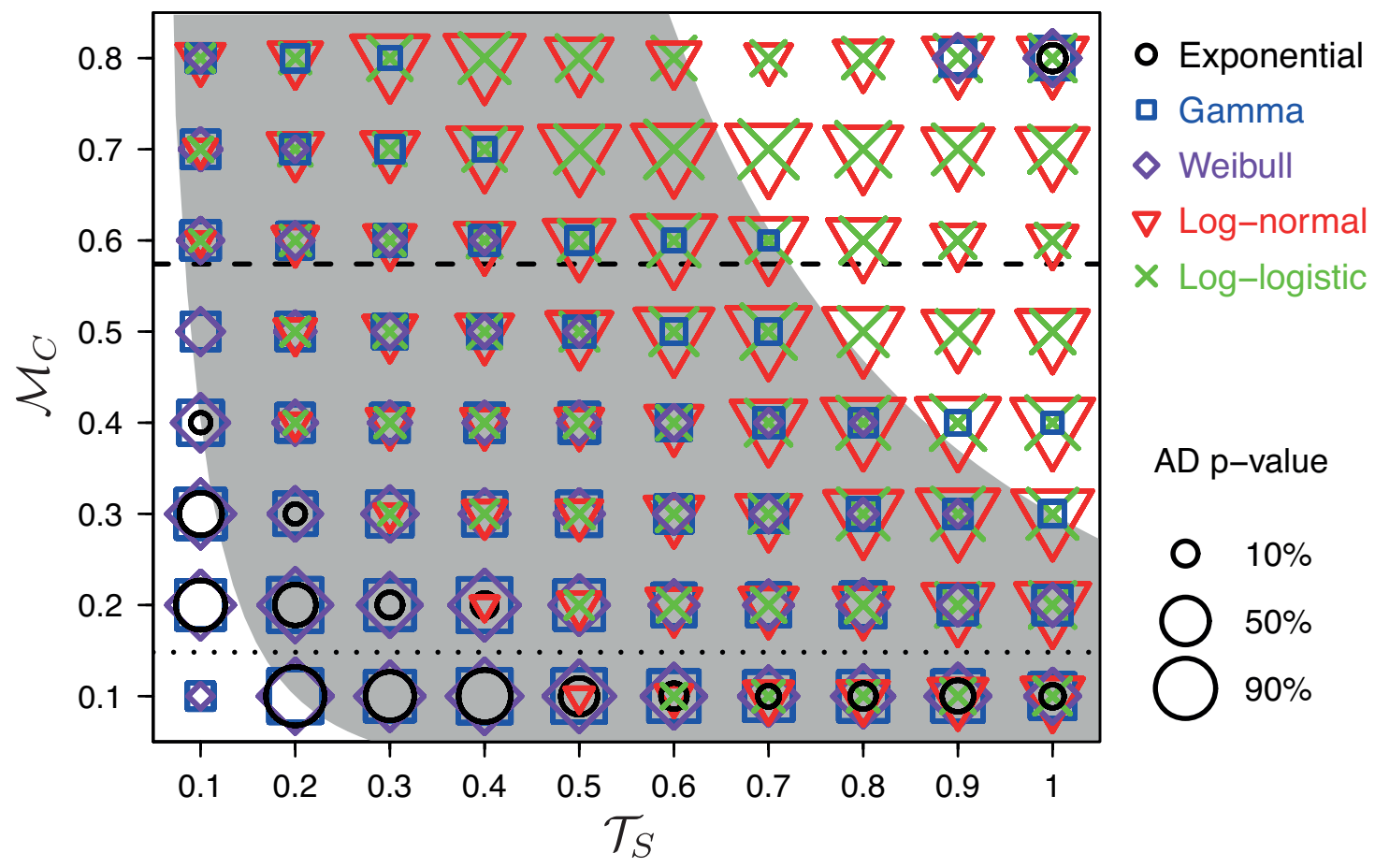

Figure 5.37: Anderson-Darling (AD) p-values of the distribution fits of the chron durations $C$ obtained for the threshold values $\mathcal{M}_{C}$ and $\mathcal{T}_{S}$ explored. The tested distribution functions are marked with different colored symbols (top right legend). Symbol sizes are scaled with the associated AD p-value (bottom right legend). Empty spaces occur where all the distribution functions can be rejected at the 5\% significance level. The dashed (dotted) horizontal line marks one (two) standard deviation(s) below the time-averaged dipole moment value. The gray background highlights parameter combinations expected to give reliable event definitions. 
bution fits for the threshold combinations of $\mathcal{M}_{C}$ and $\mathcal{T}_{S}$ explored, while the associated posterior model probabilities are reported in Figure 5.38, The statistical properties of the chron durations $C$ significantly differ from those obtained for the exponentially distributed SPI durations $\mathcal{P}$ (cf. Figures 5.15 and 5.18). In particular, the posterior model probabilities uncover gradual transitions towards different statistical models. Positive and strong evidences in favor of the exponential distribution are found only for low values of $\mathcal{M}_{C}$ and $\mathcal{T}_{S}$ where the event definitions are less reliable. Positive evidences in favor of the gamma distribution are obtained for moderate $\mathcal{M}_{C}$ and intermediate $\mathcal{T}_{S}$ values. The evidence in favor of the log-normal distribution becomes progressively larger when increasing $\mathcal{M}_{C}$. Moreover, the log-normal and log-logistic distributions systematically reach comparable posterior probabilities when increasing $\mathcal{T}_{S}$.

To gain insight into the effect of reversal durations on the polarity intervals statistic, we proceed with a closer comparison of the distributions of the SPIs $\mathcal{P}$ and the chrons $C$ for the threshold combinations $\mathrm{A}\left(\mathcal{M}_{C}=0.2, \mathcal{T}_{S}=0.1\right)$ and $\mathrm{B}\left(\mathcal{M}_{C}=0.3, \mathcal{T}_{S}=0.2\right)$ discussed in Section 5.4.1. The posterior densities of the gamma distribution parameters $k$ and $\lambda$ for the threshold combinations selected are presented in Figure 5.39 (left panels). Consider, in first place, the threshold combination A. A comparison with the respective

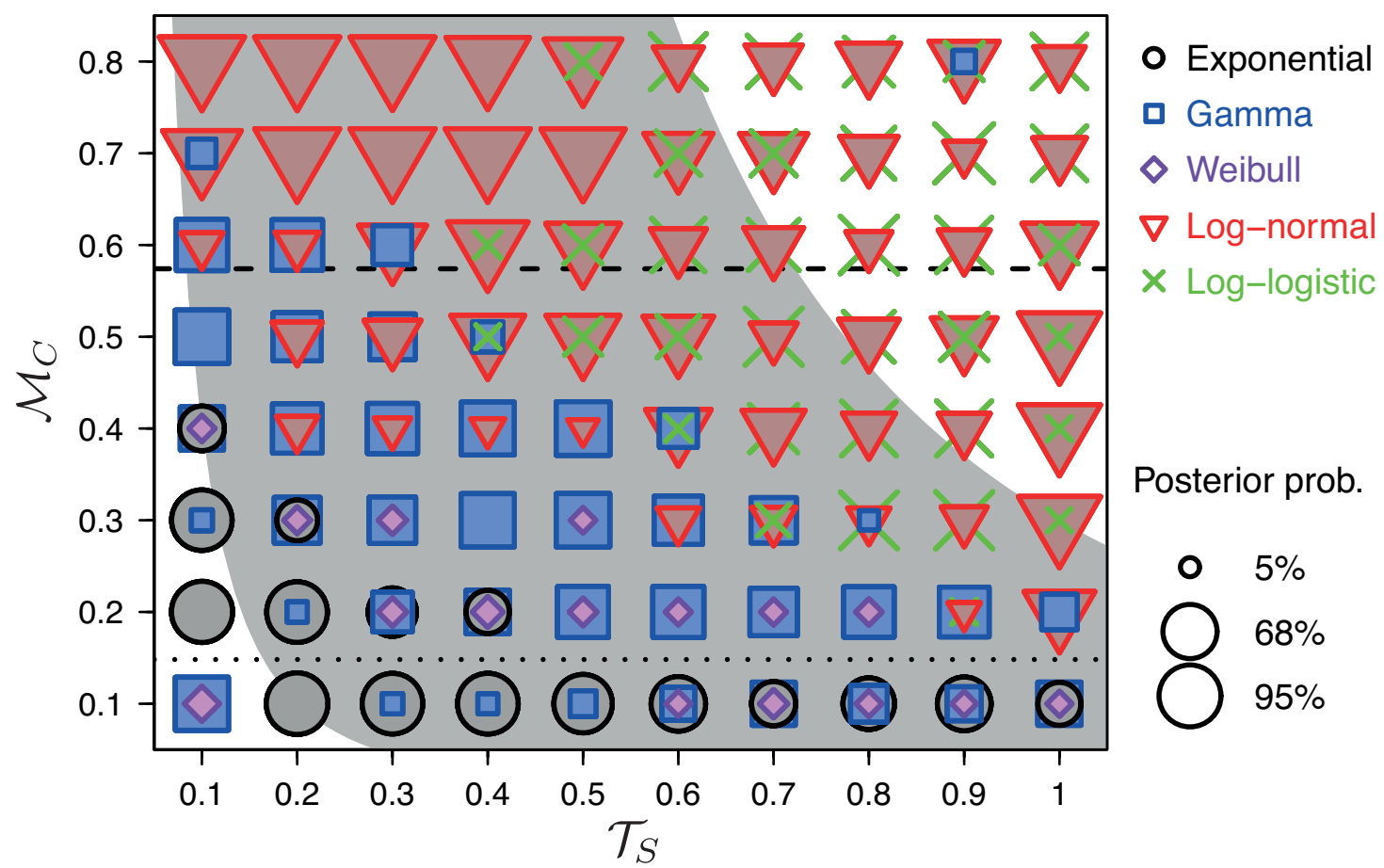

Figure 5.38: Bayesian posterior probabilities of the distribution functions (3.103) for the chron durations $C$ obtained for the threshold values $\mathcal{M}_{C}$ and $\mathcal{T}_{S}$ explored. The tested distribution functions are marked with different colored symbols (top right legend). Symbol sizes are scaled with the associated posterior probability (bottom right legend). Empty spaces occur where all the distribution functions have been rejected at the $5 \%$ significance level (cf. Figure 5.37). The dashed (dotted) horizontal line marks one (two) standard deviation(s) below the time-averaged dipole moment value. The gray background highlights parameter combinations expected to give reliable event definitions. 

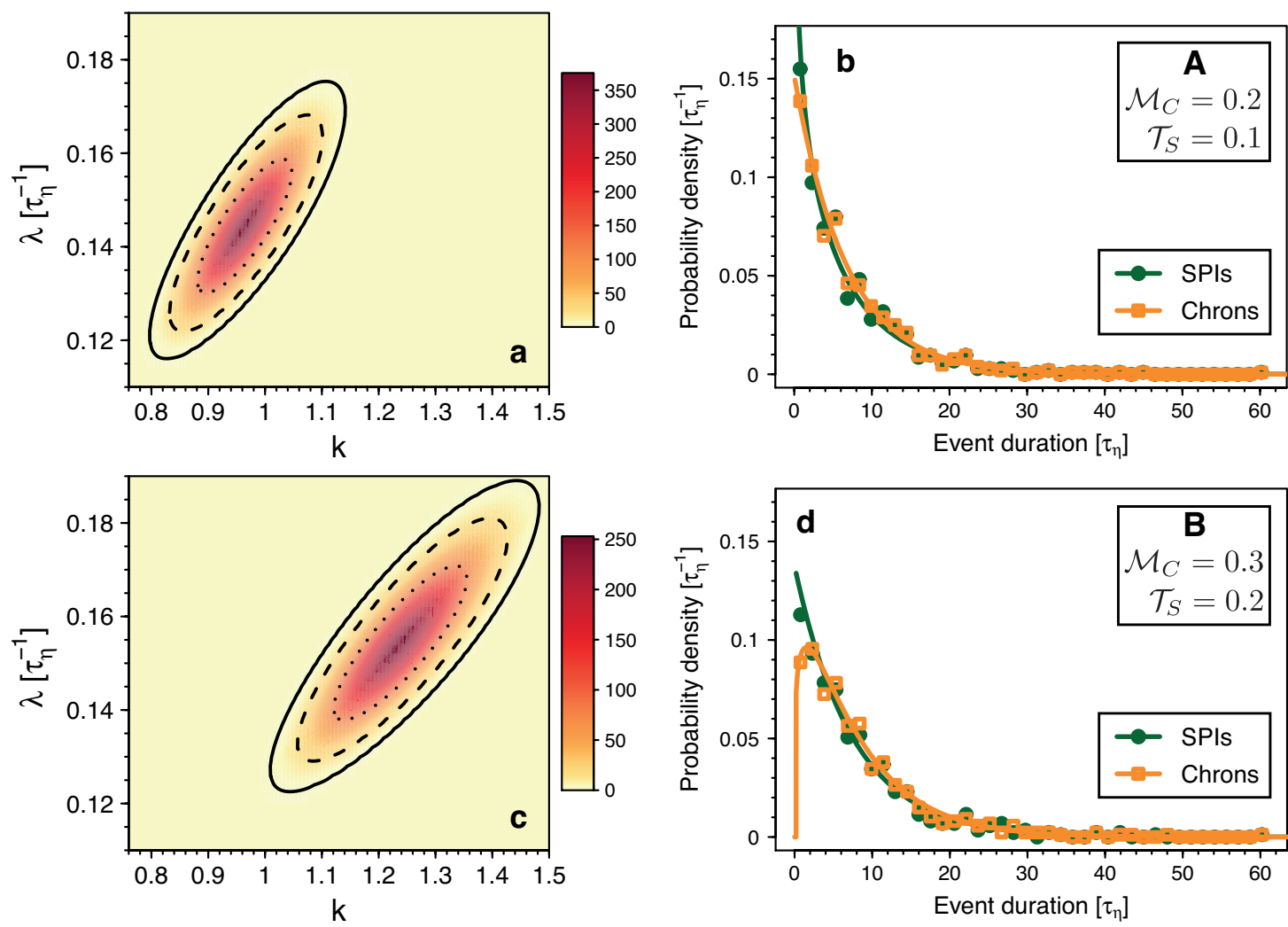

Figure 5.39: Left panels: Posterior densities of the gamma distribution parameters $k$ and $\lambda$ for the chrons $C$ obtained for the threshold combination $\mathrm{A}\left(\mathcal{M}_{C}=0.2, \mathcal{T}_{S}=0.1\right)$ and $\mathrm{B}\left(\mathcal{M}_{C}=0.3, \mathcal{T}_{S}=0.2\right)$ are shown in panels a and $\mathrm{c}$ respectively. Iso-contour lines mark the $68 \%, 95 \%$ and $99.7 \%$ highest posterior density intervals respectively. Right panels: Probability density functions of the durations of the SPIs $\mathcal{P}$ and chrons $C$ are shown for the two threshold combinations $\mathrm{A}$ and $\mathrm{B}$ in panels $\mathrm{b}$ and $\mathrm{d}$ respectively. Colored curves present the distribution fits with the highest posterior model probabilities (cf. Figures 5.18 and (5.38).

posterior density for the SPI durations $\mathrm{P}(k, \lambda \mid \mathcal{P}$, Gamma) (Figure 5.20, panel a) shows that the shape parameter $k$ significantly shifts towards larger values when the reversal durations are taken into account, while the rate $\lambda$ remains mostly unchanged. The $68 \%$ highest posterior density interval of $\mathrm{P}(k, \lambda \mid \mathcal{R}$, Gamma) includes $k=1$ (Figure 5.39, panel a), in agreement with a posterior model probability for the exponential distribution as high as $92 \%$. The combination of SPIs and reversals evidently promotes a distribution with relatively fewer short intervals. It can reasonably be assumed that the durations of the SPIs and their bounding reversals are independent. Short or long SPIs are equally likely paired with fast or slow reversals. Since the relative effect is larger for the short than for the long SPIs, the pairing tends to depopulate the short duration end of the distribution of the SPIs (Figure 5.39, panel b). This naturally favors the less steep decreasing exponential PDF. The posterior odds ratio (3.106) of the gamma to the exponential distributions reduces indeed from a value larger than 300 , obtained for the SPI durations $\mathcal{P}$, to about $1 / 20$ for the respective chron durations $C$.

The threshold combination B, with exponentially distributed SPIs, shows chrons $C$ 
best described by a gamma distribution with shape $k>1$, once more because the short duration end is depopulated (cf. Figure 5.39, panel d). Furthermore, the shape parameter value $k=1$ is excluded from the $99.7 \%$ highest posterior density interval (Figure 5.39, panel c). However, the posterior model probability of the gamma distribution is $60 \%$ and does not decisively favors this model compared to the exponential which achieves a probability of $33 \%$.

As already noticed above, the log-normal and log-logistic distributions systematically achieve the largest posterior probabilities among the selected models when increasing $\mathcal{T}_{S}$. Figure 5.40 illustrates the effect of progressively increasing $\mathcal{T}_{S}$ on the event definitions for a fixed critical dipole moment of $\mathcal{M}_{C}=0.3$. The stable period $\mathcal{T}_{S}$ varies from 0.2 (corresponding to the threshold combination B analyzed above) to the moderate value of 0.4 and finally reaches the high value of 0.9 . The distributions of the respective chron durations $C$ are shown in the right panels of Figure 5.40 together with the distribution fits achieving the largest posterior probabilities (cf. Figure 5.38). Since the threshold combination B seems to give the more reliable event definitions, it is taken as a reference case here. As $\mathcal{T}_{S}$ increases to 0.4 , the event definitions become questionable in some cases. In particular, certain 'proper' stable polarity intervals are regarded as grand excursions (see, e.g., the events occurring at times $2940 \tau_{\eta}$ and $2985 \tau_{\eta}$ in Figure 5.40) and this has been identified as problem P4 in Section 5.3. Since the likelihood of short chron durations necessarily diminishes compared to the threshold combinations with milder $\mathcal{T}_{S}$ values, the evidence in favor of the gamma distribution becomes positive with a posterior probability of $\mathrm{P}(\mathrm{Gamma} \mid C)=0.87$ (see also Figure 5.38).

When further increasing $\mathcal{T}_{S}$ to 0.9 , SPIs characterized by a large recovery in dipole intensity are affected by problem P4 (see, e.g., the events occurring at times $2968 \tau_{\eta}$ and $3000 \tau_{\eta}$ in Figure 5.40). Moreover, as discussed in Section 5.4.2, reversal durations become unreasonably long. The likelihood for longer chrons is therefore strongly enhanced and leads to a different statistic which favors the (heavy-tailed) log-normal and log-logistic distribution functions (Figure 5.29, panel f). The log-logistic achieves the largest posterior probability with $\mathrm{P}(\operatorname{LogLogistic} \mid C)=0.76$, while the log-normal has $\mathrm{P}(\operatorname{LogNormal} \mid C)=0.17$. Posterior odds ratios of the latter two models to the gamma distribution are 10.9 and 2.4 respectively. Therefore, the evidence in favor of the loglogistic and log-normal distributions is positive.

Neglecting shorter stable polarity intervals using larger $\mathcal{T}_{S}$ values mimics the unavoidable problem of paleomagnetic reversal chronologies in missing shorter geomagnetic chrons (see Section 1.4.5). Large $\mathcal{T}_{S}$ values may therefore yield distributions which can be more meaningfully compared with paleomagnetic observations of field polarities. In Chapter 4 we demonstrated that log-normal and log-logistic distributions best describe the paleomagnetic chron durations as recorded by the most recent geomagnetic polarity time scales. These results confirm previous findings obtained using different paleomagnetic reversal chronologies (see, e.g., Ryan and Sarson 2007, Shcherbakov and Fabian 2012). The numerical simulations suggest that different contributions may lead to such statistics. The first contribution reflects the likelihood of reversal occurrences which has been found to be well-described by a Poisson process (Section 5.4.1). Taking into account the finite reversal durations leads to gamma distributed chrons $C$ with shape parameters $k>1$. This introduces a period of inhibition immediately following a reversal which reflects the time required to rebuild a stable dipole field with opposite polarity. Heavy-tailed distribution 
functions, such as the log-normal and the log-logistic, finally result when artificially neglecting shorter SPIs. 

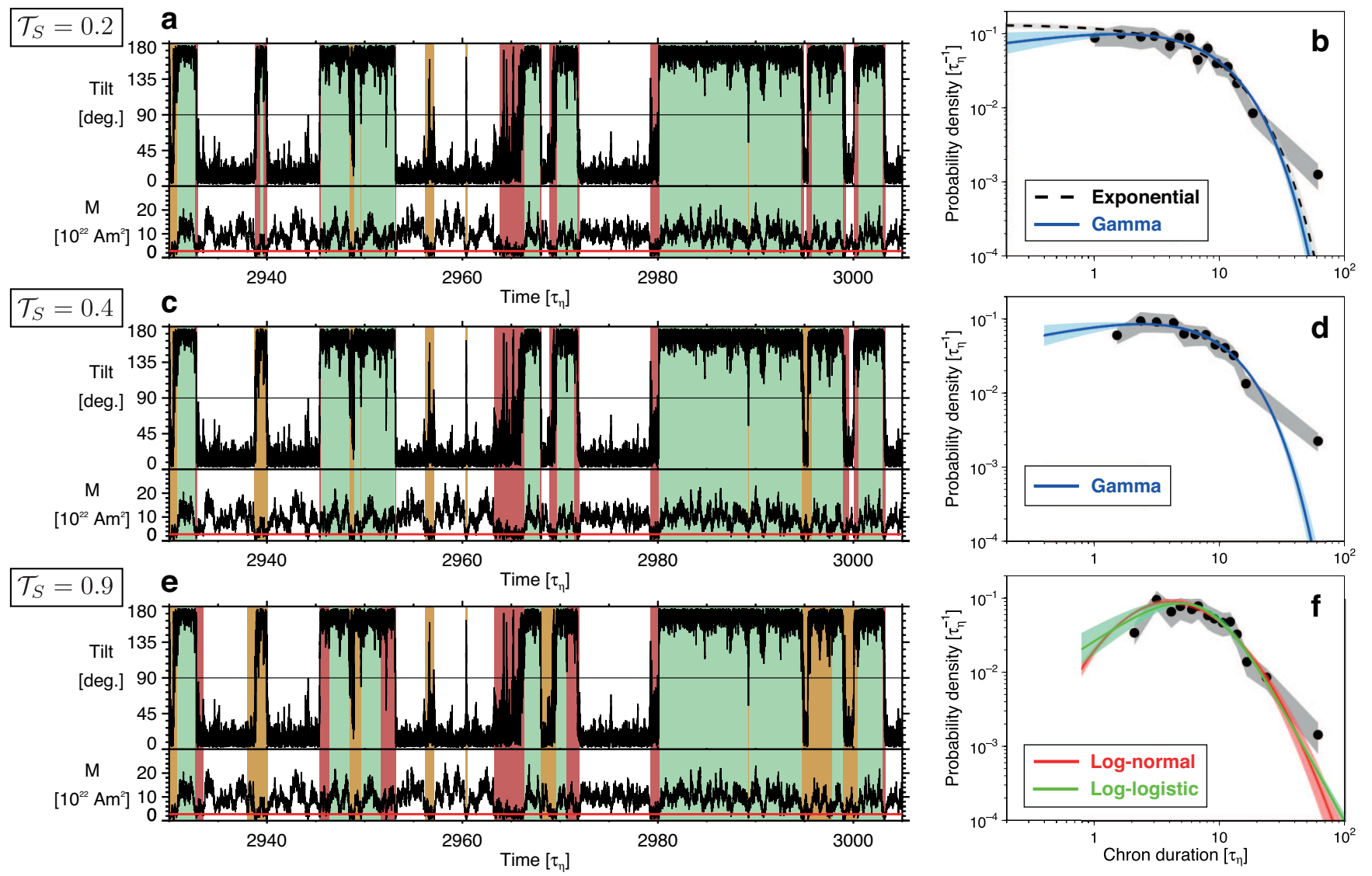

Figure 5.40: Effect of increasing the stable period $\mathcal{T}_{S}$ on the dipole tilt and dipole moment $M$ time series (left panels) and on the distributions of chron durations $C$ (right panels). The dipole moment threshold has been fixed to $\mathcal{M}_{C}=0.3$ (red horizontal line in the bottom sub-panels on the left) and the stable periods $\mathcal{T}_{S}$ are 0.2 (panels a and b), 0.4 (panels c and d) and 0.9 (panels e and f). The data probability densities are obtained using a fixed number of chrons $N$ in each bin $(N=40$ in panels b and $\mathrm{d}$, and $N=30$ in panel $\mathrm{f})$ with the grey shaded area marking the $95 \%$ confidence interval of the estimated densities. Colored curves denote the distribution fits achieving the largest posterior probabilities (see the legend insets in panels b, $\mathrm{d}$ and $\mathrm{f}$ ) and the color shaded regions mark the $95 \%$ confidence intervals of the respective fits. 



\section{Conclusions and outlook}

Polarity reversals are the most striking features of the Earth's dipolar magnetic field evolution but still remain poorly understood phenomena. Self-consistent numerical models of the geodynamo successfully show stochastic dipole field reversals in a narrow range of their input parameters. Detailed studies of polarity transitions in such models only partially helped in determining the fluid dynamic mechanisms responsible for their occurrence due to the inherent complexity of the non-linear processes involved (see, e.g., Wicht and Olson 2004, Aubert et al. 2008). The statistical study of reversals aims to provide robust constraints on the physical processes underlying their occurrence.

In this work, we studied the statistical properties of reversals and excursions in two numerical dynamo models with different Ekman numbers and Rayleigh numbers. In order to obtain robust statistical results, these models have been run for several thousand magnetic diffusion times and undergo several hundred reversals. Since the model with the lower Ekman number of $E=10^{-3}$ (model M2) more closely resembles the Earth (see the discussion in Section 5.1, we focused on the analysis of this numerical dynamo.

The simulated reversals and excursions have been identified using a criterion based on the dipole field intensity coupled with a temporal requirement on the stability of the nontransitional phases (see Section 5.3). Since the selection of specific threshold values for these criteria is somewhat arbitrary, we performed a systematic study to attest the validity of the statistical results.

We tested different distribution functions, characterizing diverse random processes as outlined in Section 3.4.1 in order to describe the statistics of the stable polarity intervals (SPIs) and the durations of reversals and excursions in our numerical simulation. We quantified the reliability of each statistical model relative to the selected alternatives using a Bayesian approach (Section 3.7).

The exponential distribution has been found to describe the SPI durations (defined excluding the bounding reversal transitions) with posterior model probabilities larger than $95 \%$. Though not rigorously tested, it seems reasonable to assume that reversals are independent events in the numerical simulation. The slowest time scale of core processes is the free dipole decay time $\tau_{d}=r_{o}^{2} / \pi^{2} \eta \approx 56 \mathrm{kyr}$, where $r_{o}$ is the outer core radius and the magnetic diffusivity $\eta$ has been calculated according to the most recent estimates of the core electrical conductivity by Pozzo et al. (2012). This time scale might be considered as an upper bound for the correlation time of core processes. Since the mean SPI duration in our simulations is $20-25$ times larger than $\tau_{d}$, the independence assumption is reasonably justified. Moreover, studies performed on both stable dipolar and reversing geodynamo models showed that dipole correlation times are typically a fraction of $\tau_{d}$ (Hulot et al. 2010, Lhuillier et al. 2011, Lhuillier et al. 2013).

Considering therefore reversals as independent events, we can conclude that a Poisson 
process best describes their occurrence in the numerical simulation. Among the different random processes tested in our analysis, the Poisson is the only memoryless process (Section 3.4.2). This implies that a reversal can start at any time during a stable polarity epoch always with equal likelihood. Long-term variations in the geodynamo models setup, for example triggered by changes in the boundary conditions in order to mimic the mantle influence (see, e.g., Driscoll and Olson 2011), could change the reversal likelihood over time and thus lead to different conclusions. However, it is not clear to which degree such variations influence the internal core dynamics and if they are necessarily required to account for the observed geomagnetic behavior (Hulot and Gallet 2003, Jonkers 2003, 2007, Biggin et al. 2012).

The other fundamental aspect regarding dipole moment variations addressed in this work concerns the equivalence of reversals and a particular subset of excursions, called grand excursions here. Grand excursions, similarly to reversals, have been defined using a criterion based both on the dipole moment intensity and on large tilts which have to venture in the opposite hemisphere (Section 5.3). The fulfillment of these criteria ensures the global character of these events (Wicht 2005). The number of reversals and grand excursions identified in model M2 have been found to be comparable. Moreover, our Bayesian analysis showed that a gamma distribution is the most likely model for the durations of both types of events. The gamma distribution parameters $k$ and $\lambda$ are furthermore equal in the limits of statistical errors (Section 5.4.4). We therefore concluded that reversals and grand excursions stem from the same underlying process. This process consists, first, in a decrease of the dipole intensity which allows for large angular deviations of the magnetic pole. After lingering in this low dipole field stage for time periods significantly shorter than the free dipole decay time $\tau_{d}$, the dipole finally recovers in intensity and thus leads to an excursion or a reversal with equal chance.

Paleomagnetic studies have documented several excursions during the most recent Brunhes and Matuyama chrons (see, e.g, Laj and Channell 2007, Valet et al. 2008). Most of these events are characterized by large angular deviations of the magnetic pole, typically larger than $45^{\circ}$, and they are correlated with major dipole intensity dips. Contrary to our results, geomagnetic excursions therefore seem to be much more frequent than reversals. However, globally correlated excursions represent only a minor fraction of the total number of events. For example, six global excursions occurred during the most recent Brunhes chron, while at least five others have not been correlated worldwide (Dormy et al. 2000). If the constraint used on the dipole strength during excursions is relaxed, 'excursion-like' events become much more numerous than reversals in our numerical simulation, thus reconciling with paleomagnetic observations. In this larger dataset, excursion durations continue to be gamma distributed but the estimated distribution parameters are significantly biased by the large number of shorter events introduced. These additional events, however, may not all represent global excursions since they are characterized by milder dipole moment variations (Wicht 2005).

We paid particular attention to the comparison of the numerical simulation results with the statistic of geomagnetic polarity intervals. The Bayesian analysis presented in Chapter 4 provided a significant evidence in favor of the (heavy-tailed) log-normal and log-logistic distributions for the chron durations as recorded by different geomagnetic polarity time scales for the past $170 \mathrm{Myr}$. This result agrees with previous studies performed in a frequentist framework and relying on different geomagnetic reversal chronologies (see, e.g., 
Ryan and Sarson 2007, Shcherbakov and Fabian 2012).

Long paleomagnetic records of reversals provide only the approximate times at which the geomagnetic field reversed its polarity and, consequently, the bounding transitional periods contribute to the duration of each stable polarity epoch. In order to mimic the paleomagnetic data set, we analyzed the simulated polarity epochs including the durations of the respective bounding reversals. Though reversal transitions are much shorter than stable polarity intervals on average, their influence on the statistic is substantial. Contrary to the exponentially distributed SPIs, polarity epochs including reversal transitions are indeed best described by a gamma distribution (Section 5.5). The finite reversal durations introduce a period of inhibition immediately following each reversal which results in estimates of the gamma shape parameters $k$ larger than one. This period reflects the time required by the internal dynamo processes to build a stable dipole field with opposite polarity and, despite largely varying from event to event, it typically amounts to a fraction of the free dipole decay time.

Due to the poor resolution of paleomagnetic data, several of the briefer geomagnetic chrons (typically shorter than $30 \mathrm{kyr}$ ) are certainly not included in the polarity time scales. We investigated the problem of missing events in our simulated reversal sequences by artificially neglecting stable polarity intervals shorter than a given period $\mathcal{T}_{S}$. We demonstrated that the gamma distributed polarity epochs including reversal transitions are progressively biased towards log-normal and log-logistic distributions when increasing $\mathcal{T}_{S}$, thus recovering the statistic of paleomagnetic chrons. Polarity intervals shorter than at least $0.7-0.8$ magnetic diffusion times $\tau_{\eta}$ have to be discarded in dynamo M2 to observe this bias. Accounting for the fact that the magnetic Reynolds number of model M2 is 5 times smaller than the Earth's value, the critical polarity interval duration estimated above amounts to $30-40 \mathrm{kyr}$ and agrees with the typical duration of shorter chrons (or cryptochrons) likely discarded. The numerical simulation therefore suggests that deviations from poissonianity in the paleomagnetic record could be the consequence of the limited data quality.

Even though log-normal and log-logistic distributions provide a good fit of paleomagnetic chron durations, they significantly underestimate the likelihood for the Cretaceous normal superchron (CNS) as shown in Section 4.2. This extremely long period of stable field polarity is not unique in the past geomagnetic history but there are evidences for two older superchrons (see Section 1.4.4). The question whether these extreme events are simply outliers or stem from the large variety of time scales produced by the geodynamo processes is still a matter of debate. Long-term variations in the reversal rate, and ultimately superchron states, have been successfully linked to external effects influencing the magnetic field core dynamics and are typically attributed to changes in the Earth's mantle (McFadden and Merrill 1984, Driscoll and Olson 2011, Biggin et al. 2012). Alternative hypotheses suggest that stochastic mechanisms resulting from the highly non-linear internal dynamo processes may naturally trigger superchrons (Hulot and Gallet 2003, Jonkers 2003, 2007). Some additional studies point towards a non-poissonian occurrence of the geomagnetic reversals. Carbone et al. (2006), for example, demonstrated that variations in the geomagnetic reversal rate are statistically incompatible with a non-stationary Poisson process. Olson et al. (2014) also report evidences for deviations in the stochastic nature of geomagnetic reversals caused by nearly periodic occurrences during certain periods.

Figure 6.1 (panel a) illustrates the variation in the paleomagnetic reversal rate ob- 
tained using a sliding window with a fixed number of reversals. The reversal rate rapidly decreases before the CNS and increases afterwards. During such periods, hints for the clustering of particularly long and very short polarity intervals seem to be present (see also Carbone et al. 2006, Jonkers 2007).

Figure 6.1 (panels b and c) also shows the same type of analysis performed on the simulated reversal sequence from the dynamo model M2. Panel b depicts a period with roughly the same number of reversals as the paleomagnetic record and contains the longest polarity epochs. The reversal rate varies by less than a factor two throughout the simulation run, while variations in the paleomagnetic record are nearly an order of magnitude larger. The longest polarity epoch in the numerical simulation (dark gray background in Figure 6.1 panels $\mathrm{b}$ and $\mathrm{c}$ ) is much shorter than the CNS. Its duration is of about 60 magnetic diffusion times or $14.4 \mathrm{Myr}$ which is $30 \%$ longer than the second longest event and 8 - 9 times longer than the mean polarity interval duration. The CNS, lasting for about $35 \mathrm{Myr}$ or 146 magnetic diffusion times, is about 6 times longer than the second longest chron and 70 times longer than the mean chron duration. This extremely long period of stable field polarity can only be reached with additional outer forcing, for example, allowing for variations in the outer boundary heat-flux (Driscoll and Olson 2011).

The lower panel of Figure 6.1 shows the reversal rate variations throughout the whole simulation run (solid curve). We also generated a synthetic sequence of polarity epochs drawing random samples from an exponential distribution. The synthetic data set consists of the same number of events identified in the numerical simulation $(N=571)$ occurring at a rate which equals the estimated exponential rate $\left(\lambda=0.134 \tau_{\eta}^{-1}\right)$. The reversal rate variations in the synthetic data set (dotted curve in Figure 6.1, panel c) are totally comparable with those obtained in the numerical dynamo simulation. This further confirms that a stationary Poisson process correctly capture the occurrence of reversals in the numerical dynamo model analyzed here (Section 5.4.1). Moreover, the longest polarity epoch identified in the numerical simulation is compatible with the fluctuations allowed by the statistical model, while the probability of occurrence of a superchron-like event remains extremely low at $4 \times 10^{-10}$. However, some degree of clustering and and periods with a more regular reversal occurrence seem to characterize the numerical simulation. In particular, it is remarkable that most of the longer polarity epochs occur in the vicinity of the longest event. The statistical analysis performed in this work cannot characterize, for example, the clustering of events. The possible non-poissonian behavior of the system on shorter time scales will be the focus of a future study. 

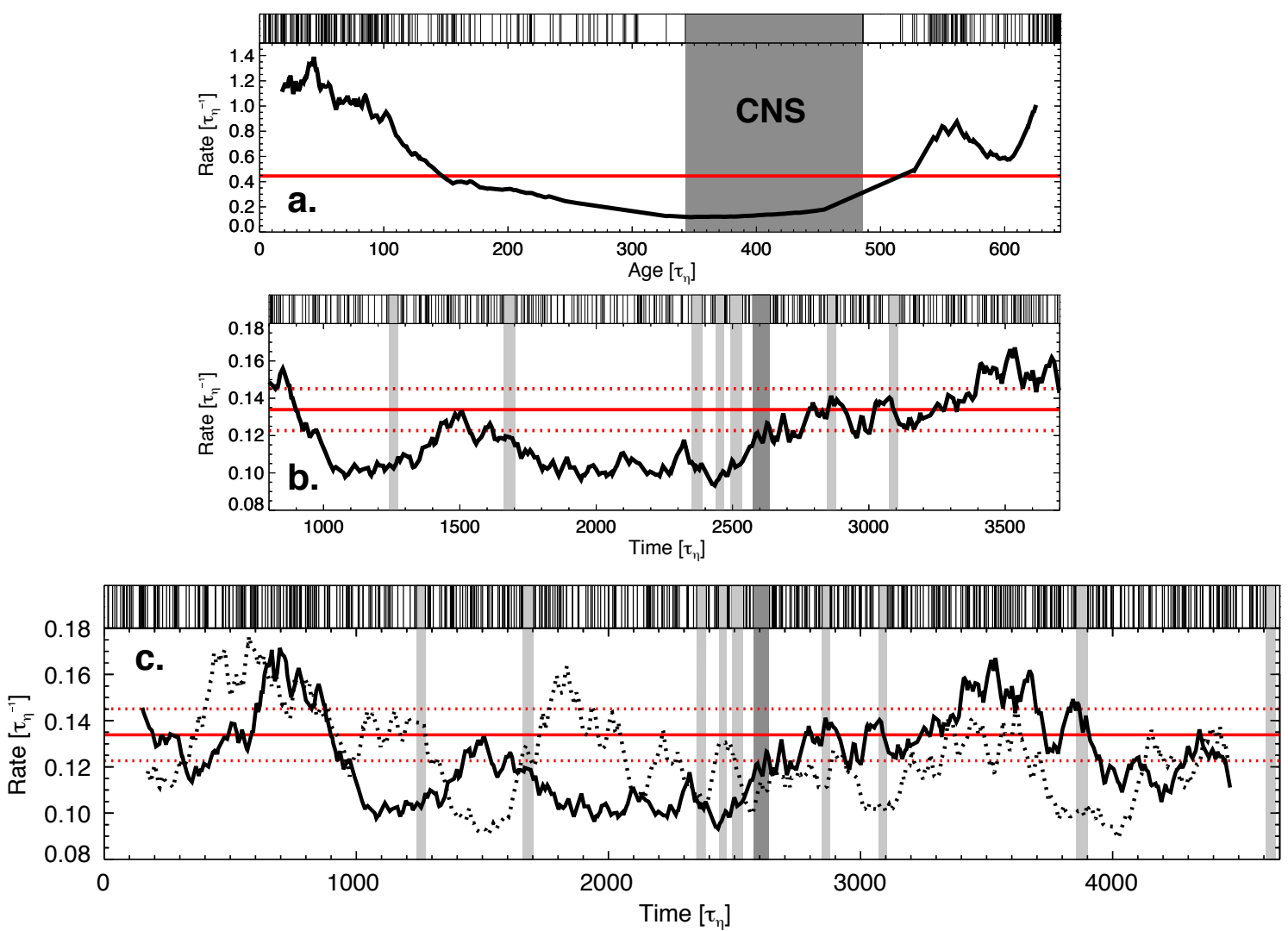

Figure 6.1: Panel a: Geomagnetic reversal rate during the past $158 \mathrm{Myr}$ from the polarity time scale of $\mathrm{Ogg}(2012)$. Time has been rescaled using the outer core magnetic diffusion time $\tau_{\eta}=244 \mathrm{kyr}$ based on the electrical conductivity estimates of Pozzo et al. (2012). The Cretaceous normal superchron (CNS) is highlighted in gray. The horizontal line shows the mean reversal rate. Panels $b$ and $c$ : Reversal rate throughout the simulation run of model M2 for a period containing roughly the same number of reversals as the paleomagnetic record (panel b) and for the complete run (panel c). The solid horizontal line represents the estimated rate from the exponential distribution fit of the stable polarity intervals obtained using the threshold combination $\mathcal{M}_{C}=0.3, \mathcal{T}_{S}=0.2$. Dotted lines mark the $95 \%$ confidence interval of the rate estimate. The dotted curve in panel c shows the reversal rate for a synthetic data set obtained drawing random samples from an exponential distribution with the above rate. Colored backgrounds highlight the longest polarity interval (dark gray) and polarity intervals longer than $30 \tau_{\eta}$ (light gray). All the reversal rate estimates have been calculated using a sliding window with a fixed number of $N=40$ reversals. 



\section{Bibliography}

Amit, H., Leonhardt, R., Wicht, J., 2010, Polarity Reversals from Paleomagnetic Observations and Numerical Dynamo Simulations, Space Science Reviews, 155, 293-335

Aubert, J., Aurnou, J., Wicht, J., 2008, The magnetic structure of convection-driven numerical dynamos, Geophysical Journal International, 172, 945-956

Backus, G., Parker, R., Constable, C., 1996, Foundations of Geomagnetism, Cambridge University Press, New York

Batchelor, G. K., 2000, An Introduction to Fluid Dynamics, Cambridge University Press, Cambridge

Biggin, A. J., Steinberger, B., Aubert, J., Suttie, N., Holme, R., Torsvik, T. H., van der Meer, D. G., van Hinsbergen, D. J. J., 2012, Possible links between long-term geomagnetic variations and whole-mantle convection processes, Nature Geoscience, 5, $526-533$

Brandt, S., 1997, Statistical and Computational Methods in Data Analysis, Springer, New York

Brunhes, B., 1906, Recherches sur la direction de l'aimantation des roches volcaniques, Journal de Physique, 5, 705-724

Bullard, E. C., Freedman, C., Gellman, H., Nixon, J., 1950, The westward drift of the earth's magnetic field, Philosophical Transactions of the Royal Society of London A: Mathematical, Physical and Engineering Sciences, 243, 67-92

Busse, F., Dormy, E., Simitev, R., Soward, A., 2007, Dynamics of rotating fluids, in Mathematical aspects of natural dynamos, (Eds.) E. Dormy, A. Soward, The Fluid Mechanics of Astrophysics and Geophysics, chap. 3, CRC Press

Cande, S. C., Kent, D. V., 1995, Revised calibration of the geomagnetic polarity timescale for the late cretaceous and cenozoic, Journal of Geophysical Research, 100, 6093-6095

Carbone, V., Sorriso-Valvo, L., Vecchio, A., Lepreti, F., Veltri, P., Harabaglia, P., Guerra, I., 2006, Clustering of Polarity Reversals of the Geomagnetic Field, Physical Review Letters, 96, 128501

Carlson, J. B., 1975, Lodestone Compass: Chinese or Olmec Primacy?, Science, 189, $753-760$ 
Chandrasekhar, S., 1961, Hydromagnetic and hydrodynamic instability, Dover, New York

Channell, J. E. T., Lehman, B., 1997, The last two geomagnetic polarity reversals recorded in high-deposition-rate sediment drifts, Nature, 389, 712-715

Channell, J. E. T., Mazaud, A., Sullivan, P., Turner, S., Raymo, M. E., 2002, Geomagnetic excursions and paleointensities in the Matuyama Chron at Ocean Drilling Program Sites 983 and 984 (Iceland Basin), Journal of Geophysical Research: Solid Earth, 107, 2114-2127

Christensen, U., Wicht, J., 2007, Numerical dynamo simulations, in Treatise on Geophysics Vol. 8: Core Dynamics, (Ed.) P. Olson, pp. 245-282, Elsevier, Amsterdam

Christensen, U., Olson, P., Glatzmaier, G. A., 1998, A dynamo model interpretation of geomagnetic field structures, Geophysical Research Letters, 25, 1565-1568

Christensen, U., Olson, P., Glatzmaier, G. A., 1999, Numerical modelling of the geodynamo: a systematic parameter study, Geophysical Journal International, 138, 393-409

Christensen, U. R., 2011, Geodynamo models: Tools for understanding properties of Earth's magnetic field, Physics of the Earth and Planetary Interiors, 187, 157-169

Christensen, U. R., Tilgner, A., 2004, Power requirement of the geodynamo from ohmic losses in numerical and laboratory dynamos, Nature, 429, 169-171

Christensen, U. R., Schmitt, D., Rempel, M., 2009, Planetary Dynamos from a Solar Perspective, Space Science Reviews, 144, 105-126

Christensen, U. R., Wardinski, I., Lesur, V., 2012, Timescales of geomagnetic secular acceleration in satellite field models and geodynamo models, Geophysical Journal International, 190, 243-254

Constable, C., 2000, On rates of occurrence of geomagnetic reversals, Physics of the Earth and Planetary Interiors, 118, 181-193

Cottrell, R. D., Tarduno, J. A., Roberts, J., 2008, The Kiaman Reversed Polarity Superchron at Kiama: Toward a field strength estimate based on single silicate crystals, Physics of the Earth and Planetary Interiors, 169, 49-58

Cowan, G., 1998, Statistical Data Analysis, Oxford University Press, Oxford

Cox, A., 1968, Lengths of geomagnetic polarity intervals, Journal of Geophysical Research, 73, 3247-3260

Cox, A., Doell, R. R., 1963, Geomagnetic Polarity Epochs and Pleistocene Geochronometry, Nature, 198, 1049-1051

D’Agostini, G., 2003, Bayesian Reasoning in Data Analysis - A Critical Introduction, World Scientific, Singapore

D’Agostino, R. B., Stephens, M. A., 1986, Goodness-of-fit techniques, Marcel Dekker, New York 
David, P., 1904, Sur la stabilitè de la direction d'aimantation dans quelques roches volcaniques, Comptes Rendus Hebdomadaires des Seances de l'Académie des Sciences (Paris), 138, 41-42

Davidson, P. A., 2001, An Introduction to Magnetohydrodynamics, Cambridge University Press, Cambridge

Dormy, E., Valet, J.-P., Courtillot, V., 2000, Numerical models of the geodynamo and observational constraints, Geochemistry, Geophysics, Geosystems, 1, 1037-42

Driscoll, P., Olson, P., 2009, Polarity reversals in geodynamo models with core evolution, Earth and Planetary Science Letters, 282, 24-33

Driscoll, P., Olson, P., 2011, Superchron cycles driven by variable core heat flow, Geophysical Research Letters, 38, 9304

Duffy, T. S., 2011, Earth science: Probing the core's light elements, Nature, 479, 480-481

Dunlop, D. J., Özdemir, Ö., 2007, Magnetizations in rocks and minerals, in Treatise on Geophysics Vol. 5: Geomagnetism, (Ed.) M. Konu, pp. 277-336, Elsevier, Amsterdam

Forbes, C., Evans, M., Hastings, N., Peacock, B., 2011, Probability distributions, John Wiley \& Sons, Hoboken, New Jersey

Gaffin, S., 1989, Analysis of scaling in the geomagnetic polarity reversal record, Physics of the Earth and Planetary Interiors, 57, 284-290

Gibbons, J. D., Chakraborti, S., 2010, Nonparametric Statistical Inference, Marcel Dekker, New York

Gilbert, W., 1600, De Magnete, Peter Short, London

Gillet, N., Jault, D., Canet, E., Fournier, A., 2010, Fast torsional waves and strong magnetic field within the Earth's core, Nature, 465, 74-77

Glatzmaier, G. A., 1984, Numerical simulations of stellar convective dynamos. I - The model and method, Journal of Computational Physics, 55, 461-484

Glatzmaier, G. A., Roberts, P. H., 1995, A three-dimensional convective dynamo solution with rotating and finitely conducting inner core and mantle, Physics of the Earth and Planetary Interiors, 91, 63-75

Gregory, P. C., 2005, Bayesian Logical Data Analysis for the Physical Sciences: A Comparative Approach with Mathematica Support, Cambridge University Press, Cambridge

Gubbins, D., 1999, The distinction between geomagnetic excursions and reversals, Geophysical Journal International, 137, F1-F4

Guyodo, Y., Valet, J.-P., 1999, Global changes in intensity of the Earth's magnetic field during the past $800 \mathrm{kyr}$, Nature, 399, 249-252 
Halley, E., 1683, A Theory on the Variation of the Magnetical Compass, Philosophical Transactions of the Royal Society of London, 13, 208-221

Halley, E., 1692, An account of the cause of the change of the variation of the magnetical needle, with an hypothesis of the structure of the internal parts of the Earth, Philosophical Transactions of the Royal Society of London, 16, 563-578

Halley, E., 1701, A new and correct chart shewing the variations of the compass in the Western and Southern Oceans as observed in year 1700, The New York Public Library, Astor, Lennox, and Tilden Foundation, New York

Hoyng, P., Ossendrijver, M. A. J. H., Schmitt, D., 2001, The geodynamo as a bistable oscillator, Geophysical and Astrophysical Fluid Dynamics, 94, 263-314

Hulot, G., Gallet, Y., 2003, Do superchrons occur without any palaeomagnetic warning?, Earth and Planetary Science Letters, 210, 191-201

Hulot, G., Lhuillier, F., Aubert, J., 2010, Earth's dynamo limit of predictability, Geophysical Research Letters, 37, L06305

Jacobs, J. A., 1994, Reversals of the Earth's magnetic field, Cambridge University Press, Cambridge

Jeffreys, H., 1961, Theory of Probability, Oxford University Press, Oxford

Johnson, N. L., Kotz, S., Balakrishnan, N., 1995, Continuous univariate distributions, Vol. 1 and 2, John Wiley \& Sons, Hoboken, New Jersey

Jones, C., 2007, Thermal and compositional convection in the outer core, in Treatise on Geophysics Vol. 8: Core Dynamics, (Ed.) P. Olson, pp. 131-185, Elsevier, Amsterdam

Jonkers, A. R. T., 2003, Long-range dependence in the Cenozoic reversal record, Physics of the Earth and Planetary Interiors, 135, 253-266

Jonkers, A. R. T., 2007, Discrete scale invariance connects geodynamo timescales, Geophysical Journal International, 171, 581-593

Kageyama, A., Miyagoshi, T., Sato, T., 2008, Formation of current coils in geodynamo simulations, Nature, 454, 1106-1109

Kolmogorov, A. N., 1933, Grundbegriffe der Wahrscheinlichkeitsrechnung, Springer, Berlin

Kono, M., 2007, Geomagnetism in perspective, in Treatise on Geophysics Vol. 5: Geomagnetism, (Ed.) M. Konu, pp. 1-33, Elsevier, Amsterdam

Korte, M., Constable, C., Donadini, F., Holme, R., 2011, Reconstructing the Holocene geomagnetic field, Earth and Planetary Science Letters, 312, 497-505

Kuang, W., Bloxham, J., 1997, An Earth-like numerical dynamo model, Nature, 389, $371-374$ 
Kutzner, C., Christensen, U. R., 2002, From stable dipolar towards reversing numerical dynamos, Physics of the Earth and Planetary Interiors, 131, 29-45

Laj, C., Channell, J. E. T., 2007, Geomagnetic excursions, in Treatise on Geophysics Vol. 5: Geomagnetism, (Ed.) M. Konu, pp. 373 - 416, Elsevier, Amsterdam

Larmor, J., 1919, How could a rotating body such as the sun become a magnet?, Report of the British Association for the Advancement of Science, pp. 159-160

Lhuillier, F., Aubert, J., Hulot, G., 2011, Earth's dynamo limit of predictability controlled by magnetic dissipation, Geophysical Journal International, 186, 492-508

Lhuillier, F., Hulot, G., Gallet, Y., 2013, Statistical properties of reversals and chrons in numerical dynamos and implications for the geodynamo, Physics of the Earth and Planetary Interiors, 220, 19-36

Lowes, F. J., 1974, Spatial power spectrum of the main geomagnetic field, and extrapolation to the core, Geophysical Journal International, 36, 717-730

Lowrie, W., 2007, Fundamentals of Geophysics, Cambridge University Press, Cambridge

Malinverno, A., Hildebrandt, J., Tominaga, M., Channell, J. E. T., 2012, M-sequence geomagnetic polarity time scale (MHTC12) that steadies global spreading rates and incorporates astrochronology constraints, Journal of Geophysical Research: Solid Earth, 117, B06104

Marzocchi, W., 1997, Missing reversals in the geomagnetic polarity timescale: Their influence on the analysis and in constraining the process that generates geomagnetic reversals, Journal of Geophysical Research, 102, 5157-5171

Matuyama, M., 1929, On the direction of magnetization of basalts in Japan, Tyosen and Manchuria, Proceedings of the Imperial Academy (Tokyo), 5, 203-205

Maus, S., Rother, M., Stolle, C., Mai, W., Choi, S., Lühr, H., Cooke, D., Roth, C., 2006, Third generation of the Potsdam Magnetic Model of the Earth (POMME), Geochemistry, Geophysics, Geosystems, 7, Q07008

McDougall, I., Tarling, D. H., 1963, Dating of Polarity Zones in the Hawaiian Islands, Nature, 200, 54-56

McElhinny, M. W., McFadden, P. L., 2000, Paleomagnetism: Continents and Oceans (International Geophysics), Academic Press, San Diego, USA

McFadden, P. L., 1984, Statistical tools for the analysis of geomagnetic reversal sequences, Journal of Geophysical Research, 89, 3363-3372

McFadden, P. L., Merrill, R. T., 1984, Lower mantle convection and geomagnetism, Journal of Geophysical Research: Solid Earth, 89, 3354-3362

McFadden, P. L., Merrill, R. T., 1993, Inhibition and geomagnetic field reversals, Journal of Geophysical Research, 98, 6189-6199 
McFadden, P. L., Merrill, R. T., 2000, Evolution of the geomagnetic reversal rate since $160 \mathrm{Ma}$ : Is the process continuous?, Journal of Geophysical Research, 105, 28455 28460

Merrill, R. T., 1998, The Magnetic Field of the Earth: Paleomagnetism, the Core, and the Deep Mantle (International Geophysics), Academic Press, San Diego, USA

Merrill, R. T., McFadden, P. L., 1999, Geomagnetic polarity transitions, Reviews of Geophysics, 37, 201-226

Naidu, P. S., 1971, Statistical structure of geomagnetic field reversals, Journal of Geophysical Research, 76, 2649-2662

Naidu, P. S., 1974, Are geomagnetic field reversals independent?, Journal of Geomagnetism and Geoelectricity, 26, 101-104

Néel, L., 1951, L'Inversion de l'aimentation permanente des roches, Annales de Geophysique, 7, 90-102

Nimmo, F., 2007, Energetics of the core, in Treatise on Geophysics Vol. 8: Core Dynamics, (Ed.) P. Olson, pp. 31-65, Elsevier, Amsterdam

Ogg, J. G., 2012, Geomagnetic polarity time scale, in Geologic Time Scale 2012, (Eds.) F. M. Gradstein, J. G. Ogg, M. Schmitz, G. Ogg, pp. 85-113, Elsevier, Boston

Olsen, N., Lühr, H., Sabaka, T. J., Mandea, M., Rother, M., Tøffner-Clausen, L., Choi, S., 2006, CHAOS-a model of the Earth's magnetic field derived from CHAMP, Ørsted, and SAC-C magnetic satellite data, Geophysical Journal International, 166, 67-75

Olsen, N., Hulot, G., Sabaka, T. J., 2007, The present field, in Treatise on Geophysics Vol. 5: Geomagnetism, (Ed.) M. Konu, pp. 33-75, Elsevier, Amsterdam

Olson, P., 2007, Overview, in Treatise on Geophysics Vol. 8: Core Dynamics, (Ed.) G. Schubert, pp. 1-30, Elsevier, Amsterdam

Olson, P., Christensen, U., Glatzmaier, G. A., 1999, Numerical modeling of the geodynamo: Mechanisms of field generation and equilibration, Journal of Geophysical Research, 104, 10 383-10 404

Olson, P., Hinnov, L. A., Driscoll, P. E., 2014, Nonrandom geomagnetic reversal times and geodynamo evolution, Earth and Planetary Science Letters, 388, 9-17

Olson, P. L., Christensen, U. R., Driscoll, P. E., 2012, From superchrons to secular variation: A broadband dynamo frequency spectrum for the geomagnetic dipole, Earth and Planetary Science Letters, 319, 75-82

Pavón-Carrasco, F. J., Osete, M. L., Torta, J. M., De Santis, A., 2014, A geomagnetic field model for the Holocene based on archaeomagnetic and lava flow data, Earth and Planetary Science Letters, 388, 98-109 
Phillips, J. D., Cox, A., 1976, Spectral analysis of geomagnetic reversal time scales, Geophysical Journal International, 45, 19-33

Phillips, J. D., Blakely, R. J., Cox, A., 1975, Independence of geomagnetic polarity intervals, Geophysical Journal International, 43, 747-754

Pozzo, M., Davies, C., Gubbins, D., Alfè, D., 2012, Thermal and electrical conductivity of iron at Earth's core conditions, Nature, 485, 355-358

Ryan, D. A., Sarson, G. R., 2007, Are geomagnetic field reversals controlled by turbulence within the Earth's core?, Geophysical Research Letters, 34, 2307

Shcherbakov, V., Fabian, K., 2012, The geodynamo as a random walker: A view on reversal statistics, Journal of Geophysical Research: Solid Earth, 117, B03101

Sivia, D. S., 1996, Data Analysis: A Bayesian Tutorial, Oxford University Press, Oxford

Spiegel, E. A., Veronis, G., 1960, On the Boussinesq Approximation for a Compressible Fluid, The Astrophysical Journal, 131, 442-447

Stern, D. P., 2002, A millennium of geomagnetism, Reviews of Geophysics, 40, 1007

Tanaka, H., Kono, M., Uchimura, H., 1995, Some global features of palaeointensity in geological time., Geophysical Journal International, 120, 97-102

Tominaga, M., Sager, W. W., 2010, Revised Pacific M-anomaly geomagnetic polarity timescale, Geophysical Journal International, 182, 203-232

Usui, Y., Tarduno, J. A., Watkeys, M., Hofmann, A., Cottrell, R. D., 2009, Evidence for a 3.45-billion-year-old magnetic remanence: Hints of an ancient geodynamo from conglomerates of South Africa, Geochemistry, Geophysics, Geosystems, 10, Q09Z07

Valet, J.-P., Meynadier, L., Guyodo, Y., 2005, Geomagnetic dipole strength and reversal rate over the past two million years, Nature, 435, 802-805

Valet, J.-P., Plenier, G., Herrero-Bervera, E., 2008, Geomagnetic excursions reflect an aborted polarity state, Earth and Planetary Science Letters, 274, 472-478

Vine, F. J., Matthews, D. H., 1963, Magnetic Anomalies Over Oceanic Ridges, Nature, 199, 947-949

Weibull, W., 1951, A statistical distribution function of wide applicability, ASME Journal of Applied Mechanics, 18, 293-297

Wicht, J., 2002, Inner-core conductivity in numerical dynamo simulations, Physics of the Earth and Planetary Interiors, 132, 281-302

Wicht, J., 2005, Palaeomagnetic interpretation of dynamo simulations, Geophysical Journal International, 162, 371-380

Wicht, J., Olson, P., 2004, A detailed study of the polarity reversal mechanism in a numerical dynamo model, Geochemistry, Geophysics, Geosystems, 5, Q03H10 
Wicht, J., Stellmach, S., Harder, H., 2009, Numerical models of the geodynamo: From fundamental cartesian models to $3 \mathrm{~d}$ simulations of field reversals, in Geomagnetic Field Variations, Advances in Geophysical and Environmental Mechanics and Mathematics, pp. 107-158, Springer, Berlin-Heidelberg

Wicht, J., Stellmach, S., Harder, H., 2010, Numerical dynamo simulations: From basic concepts to realistic models, in Handbook of Geomathematics, (Eds.) W. Freeden, M. Nashed, T. Sonar, pp. 459-502, Springer, Berlin-Heidelberg

Wilson, R. L., 1962, The Palaeomagnetism of Baked Contact Rocks and Reversals of the Earth's Magnetic Field, Geophysical Journal International, 7, 194-202 


\section{Acknowledgements}

Firstly, I would like to express my sincere gratitude to my advisor Dr. Johannes Wicht who guided me during all my PhD studies. His open-mindedness and his profound knowledge of planetary dynamos have been extraordinary helpful. His enthusiasm in tackling new problems always stimulated me and made our discussions particularly enjoyable.

I am indebted to Dr. Dieter Schmitt. As a former coordinator of the Solar System School, I firstly contacted him when I applied for my $\mathrm{PhD}$ scholarship. He believed in our project since the beginning and he played a crucial role in supporting me. I enjoyed our scientific discussions and his calm and accurate explanations of mean-field dynamos. He passed away during the last year of my PhD studies and I lost an excellent guide. This thesis is dedicated to him. I will try my best to continue our original project which, unfortunately, did not come to an end.

I thank my advisor Prof. Andreas Tilgner for his help and for being the main referee of this work. A special thanks goes to Prof. Ulrich Christensen for his guidance during the last period of my $\mathrm{PhD}$ studies. I have been always impressed by its ability in explaining complex concepts in a simple and exhaustive manner. I am grateful to Dr. Sonja Schuh, the actual coordinator of the Solar System School, for her efforts in improving the quality of the School and for the help she unconditionally gives to all the students.

I also thank all the present and former members of the dynamo group at the MPS with whom we shared interesting discussions: Lúcia, Kumiko, Rakesh, Andrea and Ankit. Special thanks go to my officemate Wieland Dietrich for all our scientific discussions on our favorite whiteboard (still missing in the new office). He has also been of great help all the times I was fined for something in Germany. Special thanks go to Thomas Gastine who always shared with me his experience and gave fundamental advices for future plans. I thank Martin Schrinner for his great help and our fruitful discussions.

During my stay at the MPS, I met wonderful people and listing all of them here would be a hard work. Special thanks go to Marc, Kok Leng, Maria D., Maria A., Vali, Gordon, Juanjo, David, Peter, Megha, Nafiseh, Don, Roberto and Nathalie. We enjoyed all the activities organized and we had a lot of fun together.

Marco Stangalini and Emanuele Papini made the past two years particularly enjoyable. We discussed everything from science to astute plans in order to survive in Lindau. With his "subtle" humour, Marco always helped in taking the best even from difficult situations. Emanuele never denied his help and support. The evenings spent together and our whiskey activities have been great especially because of his enviable spontaneity. I clap my hands for both of them. I sincerely thank Luca who never gave up for a coffee. Sharing stories from our common southern origin and his lively family always reminded me home while in Germany. I am grateful to Piero and Annalisa for the enjoyable times in front of a "Schnitzel" (and beers). 
I greatly and sincerely thank my parents Luigi and Anna, and my sister Ilaria for their love and for their unconditional support. This work would certainly not have been possible without them.

Despite all the difficulties, Giulia never ceased to be by my side and always took the best from me. The journey of our life together is everyday a new discovery where I learn what love is. During these years, she followed me with unconditional love and therefore I also dedicate this work to her. I am very grateful to my second large family in Bolzano. A very special thanks goes to Tino for making me feel home and for sharing all his great stories in front of a glass of wine. I am particularly indebted to Riky and Stefania for their support and advices during the dark periods of these years. Finally, I would like to thank Cinzia and Renato who always gave great encouragements.

"The Road goes ever on and on Down from the door where it began.

Now far ahead the Road has gone,

And I must follow, if I can, Pursuing it with eager feet, Until it joins some larger way Where many paths and errands meet. And whither then? I cannot say" J. R. R. Tolkien, The Lord of the Rings 


\section{Scientific contributions}

\section{Conferences}

- Meduri, D., Wicht, J., Sorriso-Valvo, L., Carbone, L., "A statistical analysis of reversal sequences: geomagnetic field and numerical dynamo models", European GdR Dynamo Meeting, Cargese, Corsica, 12-17 September 2011

- Meduri, D., Wicht, J., Schmitt, D., Carbone, V., Sorriso-Valvo, L., “A statistical analysis of reversal sequences: geomagnetic field, coupled spin and numerical dynamo models", EPSC-DPS Joint Meeting 2011, Nantes, France, 2-7 October 2011

- Meduri, D., Wicht, J., Schmitt, D., "Statistical analysis of stable polarity intervals in geodynamo models and its comparison with paleomagnetic data", SEDI 2012, Leeds, UK, 1-6 July 2012

- Meduri, D., Wicht, J., Schmitt, D., "Statistical analysis of reversals and stable polarity epochs in geodynamo models and paleomagnetic data", European GdR Dynamo and MHD Days, Nice, France, 1-4 October 2012

- Meduri, D., Wicht, J., Schmitt, D., "Reversals in direct numerical simulations and mean-field models of the geodynamo: a statistical approach", European GdR Dynamo Meeting, Ascona, Switzerland, 7-12 July 2013

- Meduri, D., Wicht, J., Schmitt, D., "Reversals in direct numerical simulations of the geodynamo: a statistical analysis", AGU Fall Meeting, San Francisco, USA, 9-13 December 2013 



\section{Curriculum Vitae}

\section{Personal Information}

Name

Domenico Giovanni Meduri

Date of birth October 30, 1985

Place of birth Reggio di Calabria, Italy

Nationality Italian

E-mail_ domenicomeduri@gmail.com

\section{Education}

2011-2014

PHD STUDIES

International Max Planck Research School for Solar System Science at the University of Göttingen

Institute Max-Planck-Institut für Sonnensystemforschung, Göttingen, Germany

University

Georg-August-Universität Göttingen, Germany

Title

Statistical Study of Magnetic Field Reversals in Geodynamo Models and Paleomagnetic Data

Supervisors Prof. Ulrich Christensen, PD Dr. Dieter Schmitt, Prof. Andreas Tilgner, Dr. Johannes Wicht

2007-2010 Master's degree in Astrophysics and Plasma Physics

Università della Calabria, Rende (CS), Italy

achieved on May 11, 2010

Master's Thesis

Title

The Solar Dynamo Dilemma

Supervisors

Prof. Vincenzo Carbone, Dr. Antonio Vecchio

2004-2007

Bachelor's degree in Physics

Università della Calabria, Rende (CS), Italy

achieved on October 3, 2007

Bachelor's Thesis 
Title Un modello dinamico per l'effetto dinamo: analisi delle correlazioni nelle inversioni del campo geomagnetico e nell'esperimento della macchina di Bullard-Von Kármán

Supervisors

Prof. Vincenzo Carbone, Dr. Luca Sorriso-Valvo

$1999-2004$

High School Diploma

Liceo Scientifico "L. Da Vinci”, Reggio di Calabria (RC), Italy

achieved on July 6, 2004

\section{Publications}

2012

Vecchio, A., Laurenza, M., Meduri, D., Carbone, V., Storini, M., The dynamics of the solar magnetic field: polarity reversals, butterfly diagram, and quasi-biennial oscillations, The Astrophysical Journal 749, 27 (2012)

2009

Donato, S., Meduri, D., Lepreti, F., Magnetic field reversals of the Earth: a two-disk Rikitake dynamo model, International Journal of Modern Physics B 23, 5492 (2009) 